\title{
A SEX-SPECIFIC VIEW ON CORONARY VASCULAR DISEASE
}

Floor Groepenhoff

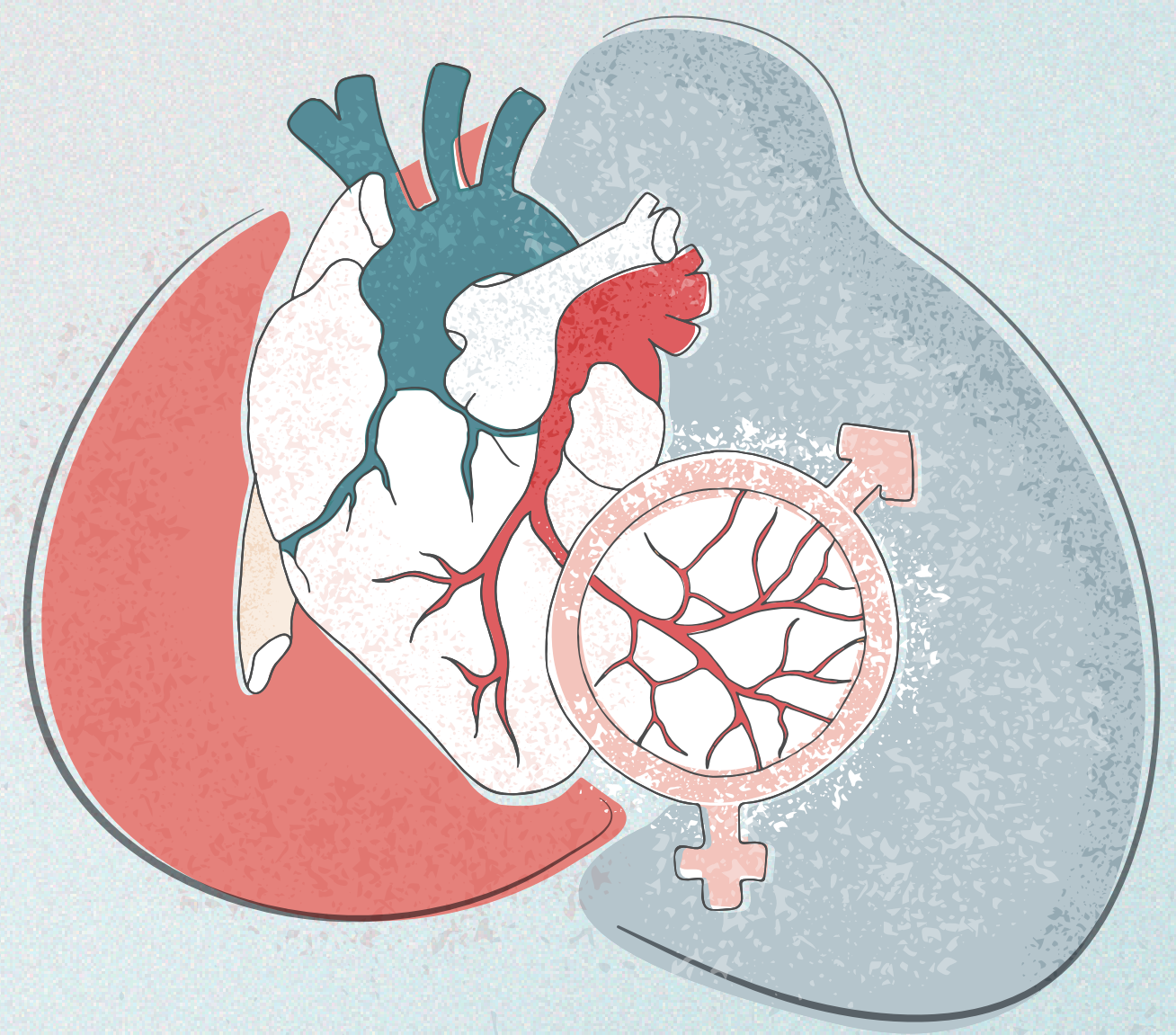





\section{A SEX-SPECIFIC VIEW ON CORONARY VASCULAR DISEASE}

Floor Groepenhoff 
A Sex-Specific View On Coronary Vascular Disease

$\mathrm{PhD}$ thesis with a summary in Dutch

Author: Floor Groepenhoff

Rights: Please use the content of this $\mathrm{PhD}$ thesis as much as possible, with appropriate references

Lay out and design: Wendy Schoneveld, www.wenziD.nl

Cover illustration: Marian Wesseling, Wendy Schoneveld, www.wenziD.nl

Printing: ProefschriftMaken, Proefschriftmaken.nl

ISBN: 978-94-6423-498-5

The research described in this thesis was supported by a grant of the Dutch Heart Foundation (DHF CVON 2017-22 ARGUS).

Financial support by the Dutch Heart Foundation for the publication of this thesis is gratefully acknowledged.

Financial support by Medis Medical Imaging Systems bv, Cardiologie Centra Nederland and ChipSoft for the publication of this thesis is gratefully acknowledged. 


\section{A Sex-Specific View On Coronary Vascular Disease}

Een geslachtsspecifieke kijk op coronair vaatlijden

(met een samenvatting in het Nederlands)

\section{Proefschrift}

ter verkrijging van de graad van doctor aan de

Universiteit Utrecht

op gezag van de

rector magnificus, prof. dr. H.R.B.M. Kummeling, ingevolge het besluit van het college voor promoties

in het openbaar te verdedigen op

dinsdag 7 december 2021 des middags te 4.15 uur

door

\section{Floor Groepenhoff}

geboren op 21 oktober 1994

te Amsterdam 


\section{Promotoren}

Prof. dr. ir. H.M. den Ruijter

Prof. dr. T. Leiner

\section{Copromotoren}

Dr. N.C. Onland-Moret

Dr. A.L.M. Eikendal 



\section{TABLE OF CONTENTS}

Chapter 1 General introduction and thesis outline

PART I SYMPTOMS

Chapter 2 Coronary artery disease prediction in women and men using chest pain characteristics and risk factors: an observational study in outpatient clinics

Chapter 3 NYHA class is strongly associated with mortality beyond heart failure in symptomatic women

PART II PATHOPHYSIOLOGY AND IMAGING

Chapter 4 Sex-specific aspects in the pathophysiology and imaging of coronary macro- and microvascular disease

Chapter 5 Cardiovascular imaging of women and men visiting the outpatient clinic with chest pain or discomfort: design and rationale of the ARGUS study

Chapter 6 Women with higher grades of coronary stenoses have lower calcium scores than men

Chapter 7 Measures for coronary artery calcification predict mortality in symptomatic women and men 
PART III SYMPTOMATIC WOMEN AND MEN WITH NON-OBSTRUCTED CORONARIES

Chapter 8 Persistent symptoms and health needs of women and men with non-obstructed coronary arteries in the years following coronary angiography

Chapter 9 Evaluation of non-invasive imaging parameters in coronary microvascular disease: a systematic review

\section{PART IV GENERAL DISCUSSION}

Chapter 10 General Discussion

\section{APPENDIX}

Samenvatting in het Nederlands

Dankwoord 188

List of publications 


$$
=1
$$




\title{
General introduction and thesis outline
}

\author{
Parts of this text have been published in Focus Vasculair (1)
}




\section{Coronary vascular disease throughout the years from a sex-specific point of view}

In recent years, sex-differences in cardiovascular research gained a lot of attention. The history that led to the current focus on sex in cardiovascular research is intriguing. Even though scientific studies addressing cardiovascular disease date back to the $18^{\text {th }}$ century, the first study in which the influence of sex was appreciated was not published until 1926. The real rise in numbers of publications conscious of the role of sex in cardiovascular disease started around the 1970's. In that period, the realization that coronary vascular disease was not just a men's problem got through to the scientific community as well as to society.

In 1975, the Dutch Heart Foundation paid attention to the role of women in the fight against cardiovascular disease (2). At that time, women were not considered to be affected as patients but had the role of the caretaker of men with ischemic heart disease. Therefore, they were given the "important" task of facilitating revalidation and providing a healthy diet for these men. Yet, there was also a progressive tone in the column of the Dutch Heart Foundation as it discussed the possible future risk of cardiovascular disease in women as well. It raised the question whether women themselves would never have to deal with cardiovascular disease, and whether smoking, the burden of mental or physical stress and a sedentary lifestyle would be without disastrous consequences for women. In addition, the article warned that, as women were pursuing a career outside of their household, the growing emotional burden coming along with this expanding role might lead to the development of cardiovascular disease in these women. At the end of the article, they concluded that the emancipation of women "should give women equal rights, not an equal risk of suffering from cardiovascular disease".

Since then, fortunately, awareness of cardiovascular disease risk in women has grown both in science and in clinical practice. This is important, as cardiovascular disease remains the number one cause of death in women, especially after menopause (3). Increased knowledge and awareness of this risk led to the development of the first women-specific American Heart Association recommendations for prevention of cardiovascular disease in 1993 (4) and to the first real guideline for women in 2011 (5). Among other things, these recommendations drew attention to the role of femalespecific risk factors such as pregnancy complications and menopause, and the possibilities offered by knowledge on these factors to prevent cardiovascular disease in women. 
In addition to awareness in science and clinical practice, awareness among women is just as important. Campaigns such as the "Dress Red Day" and the "Go Red for Women" days, initiated in 2002 and 2004 respectively, contributed raising awareness for this topic. Concluding, we have come a long way; knowledge has improved on both the biological and social aspects (6) of cardiovascular disease in women. Yet, a lot of work still needs to be done to improve prevention and care for coronary vascular disease in women: In current clinical care most 'evidence based medicine' is based on studies investigating mainly male patients and as a result both clinicians and riskassessment-models still tend to underestimate disease risk in women (7).

In this thesis, we aim to further increase the knowledge on sex-differences in the cardiovascular field, zooming in on coronary vascular disease. Coronary vascular disease comprises the complete coronary vascular tree, from epicardial coronary arteries at macro level to the coronary microvasculature at micro level.

\section{Obstructive coronary vascular disease}

Cardiovascular disease is often the result of atherosclerosis, a lifelong process of vascular damage. In coronary artery disease atherosclerosis occurs in the epicardial arteries and can become fatal if it leads to blockage of the myocardial blood supply. This form of atherosclerosis, also known as macrovascular or obstructive coronary artery disease, is particularly prevalent in men. In the 1980 s, plaque rupture and erosion were discovered as the underlying mechanisms (8). Eroded plaques appeared to be more often the cause of the occlusion in women, while in men predominantly a ruptured plaque was found (8). Plaques with a high risk of rupture have a thin fibrous cap, a high fat content and a large necrotic core underneath. A plaque rupture is more common when an unstable plaque is infiltrated by inflammatory cells or with an intra-plaque bleeding. Especially in these bleeds, various atherogenic factors, such as inflammatory cells or red blood cells, leak from the blood vessels in the plaque. These factors can accelerate the process of atherosclerosis, resulting in an unstable plaque (9). Prior to erosive coronary thrombosis, there is usually a more stable plaque phenotype, recognizable by a thick fibrous cap and absence of a necrotic core. The fibrous cap remains intact, and the core of the plaque does not come into contact with the thrombus. Both mechanisms leading to coronary thrombosis are compared in Table 1. 
Table 1. Comparison of characteristics of plaque rupture vs. erosion (1)

\begin{tabular}{ll}
\hline Plaque rupture & Plaque erosion \\
\hline Instable plaque & Stable plaque \\
More common in men & More common in women \\
Thin fibrous cap & Thick fibrous cap \\
$\begin{array}{l}\text { Necrotic core containing fat, inflammatory cells } \\
\text { and 'plaque hemorrhage' }\end{array}$ & Absence of necrotic core \\
$\begin{array}{l}\text { Occlusion of coronary artery with large thrombus } \\
\text { after plaque rupture }\end{array}$ & $\begin{array}{l}\text { Thrombocyte adhesion on an intact plaque in } \\
\text { combination with microemboli formation }\end{array}$ \\
\hline
\end{tabular}

The diagnosis of obstructive coronary artery disease is preferably confirmed with a flow measurement during an invasive coronary angiogram, but can also be made using a non-invasive computed tomography (CT) scan, i.e., cardiac CT. During cardiac $\mathrm{CT}$, first the cardiac calcium score is assessed. Subsequently, after intravenous contrast injection, the coronary arteries are examined in more detail to evaluate the absence or presence of wall abnormalities, (non-)calcified plaques and the degree of stenosis. Obstructive coronary artery disease is less common in women; however, women who do have obstructive CAD have a worse prognosis than men $(10,11)$. In women who underwent a coronary angiogram for an acute coronary syndrome, the risk of cardiovascular events, defined as heart failure, a cerebrovascular event, angina or myocardial infarction, is $44-70 \%$ higher during the 2 years following the coronary angiogram as compared to men. In women with stable coronary artery disease undergoing a coronary angiogram, this risk was $29-53 \%$ higher than in men.

\section{Ischemia without obstructive vascular disease}

In absence of obstructive coronary artery disease, myocardial ischemia can still be present. Symptomatic women more often suffer from this non-obstructive coronary vascular disease. Thus, in women with evidence of myocardial ischemia, additional research is more likely to find clean or non-obstructed coronary arteries. Up until recently, these women were "falsely" reassured that their complaints were of noncardiac origin. We now know that these symptomatic women without obstruction of the epicardial coronary arteries are more likely to have myocardial ischemia than men (10). We call this "Ischemia with Non-Obstructed Coronary Arteries", in short INOCA. Contrary to previous beliefs, these women are at increased risk of developing serious cardiovascular events (13). Recent research has shown that two third of women with persistent anginal (equivalent) symptoms have myocardial ischemia due to dysfunction of the coronary vascular system (12). Coronary vascular dysfunction can be based on coronary microvascular dysfunction and/or epicardial vasospasm. The underlying 
mechanism in these INOCA women could also be microemboli derived from nonobstructive coronary plaques leading to downstream microvascular obstruction (14). These downstream microembolic obstructions in the heart are more often seen with plaque erosion than with plaque rupture. This suggests that women may develop microembolizations during the development of stable atherosclerosis, leading to coronary microvascular dysfunction.

To include both obstructive coronary artery disease and vascular dysfunction, the term coronary vascular disease is used throughout this thesis. In this thesis we aim to provide insight in coronary vascular disease in women and men by comparing symptomatic women and men that present at the outpatient cardiology clinic. The first part evaluates the value of currently used risk-assessment tools in this heterogeneous patient population at outpatient cardiology clinics. The second part assesses pathophysiological sex differences in coronary vascular disease in this population with use of non-invasive imaging. The last part of this thesis answers the question how large the population of women and men with prolonged complaints after an exclusion diagnosis of obstructive coronary artery disease is and how these individuals at risk could be identified.

\section{Thesis outline}

The first part of this thesis focuses on the replication of symptom-based risk prediction in the outpatient cardiology clinic in a sex-stratified manner. In previous research many "evidence based" clinical tools were developed only in male patients but used in both women and men. Furthermore, even when the study populations comprised female patients as well, sex-specific reporting was still scarce. To overcome this important limitation of contemporary clinical care, Chapter $\mathbf{2}$ evaluates coronary artery disease prediction in women and men using chest pain characteristics and risk factors. Moreover, Chapter $\mathbf{3}$ shows the association of functional classification of complaints, originally developed in male heart failure patients, with mortality, in a heterogeneous female patient population presenting with a variety of complaints.

The second part studies sex differences in coronary vascular disease in women and men presenting to the outpatient clinic at the level of pathophysiology and imaging results. This part aims to expand knowledge on sex differences from histological studies in patients with advanced atherosclerotic disease to symptomatic women and men suspect of coronary vascular disease in an early stage of disease. First, Chapter 4 reviews the current state of the literature on sex-specific aspects of the 
pathophysiology and imaging of coronary vascular disease. Second, in Chapter $\mathbf{5}$ we introduce the ARGUS study in which extensive clinical phenotyping is performed by state-of-the-art cardiovascular imaging in all women and men presenting with chest pain to the outpatient cardiology clinic. Subsequently, in Chapter 6 we study the differences in the association between coronary artery calcification and severity of coronary artery disease between these women and men in the ARGUS study, as we suspect this relation to be sex-specific due to known sex-differences in plaque composition. In Chapter $\mathbf{7}$ we investigate the prognostic value of measures of coronary artery calcification as visualized on CCTA in symptomatic women and men.

Lastly, the third part focuses on the until recently underappreciated and therefore relatively unknown patient population of symptomatic women and men with nonobstructive coronary artery disease. In Chapter 8 we study whether these women and men have different care demands compared with women and men from the general population. Chapter 9 reviews existing literature on non-invasively measured parameters that could improve detection of those women and men who are at risk for adverse (cardiovascular) outcomes.

The Chapters 10 and 11 contain the general discussion and Dutch summary. 


\section{REFERENCES}

1. Groepenhoff F, van Ommen, A.M.L.N., den Ruijter, H.M. Hart- en vaatziekten bij vrouwen en mannen door de jaren heen. Focus Vasculair. 2021; Jaargang 6 - editie 1 - Editie 1, 2021.

2. Hartstichting. De Vrouw en Haar Hart. 1973.

3. El Khoudary SR, Aggarwal B, Beckie TM, Hodis HN, Johnson AE, Langer RD, et al. Menopause Transition and Cardiovascular Disease Risk: Implications for Timing of Early Prevention: A Scientific Statement From the American Heart Association. Circulation. 2020;142(25):e506-e32.

4. Mosca L, Grundy SM, Judelson D, King K, Limacher M, Oparil S, et al. Guide to preventive cardiology for women. Circulation. 1999;99(18):2480-4.

5. Mosca L, Benjamin EJ, Berra K, Bezanson JL, Dolor RJ, Lloyd-Jones DM, et al. Effectiveness-based guidelines for the prevention of cardiovascular disease in women-2011 update: a guideline from the American Heart Association. Journal of the American College of Cardiology. 2011;57(12):1404-23.

6. Mosca L, Mochari-Greenberger H, Dolor RJ, Newby LK, Robb KJ. Twelve-year follow-up of American women's awareness of cardiovascular disease risk and barriers to heart health. Circulation: Cardiovascular Quality and Outcomes. 2010;3(2):120-7.

7. Vogel B, Acevedo M, Appelman Y, Merz CNB, Chieffo A, Figtree GA, et al. The Lancet women and cardiovascular disease Commission: reducing the global burden by 2030. The Lancet. 2021.

8. Farb A, Burke AP, Tang AL, Liang Y, Mannan P, Smialek J, et al. Coronary plaque erosion without rupture into a lipid core: a frequent cause of coronary thrombosis in sudden coronary death. Circulation. 1996;93(7):1354-63.

9. Vrijenhoek JE, Den Ruijter HM, De Borst GJ, de Kleijn DP, De Vries J-PP, Bots ML, et al. Sex is associated with the presence of atherosclerotic plaque hemorrhage and modifies the relation between plaque hemorrhage and cardiovascular outcome. Stroke. 2013;44(12):3318-23.

10. Parvand M, Rayner-Hartley E, Sedlak T. Recent Developments in Sex-Related Differences in Presentation, Prognosis, and Management of Coronary Artery Disease. Canadian Journal of Cardiology. 2018;34(4):390-9.

11. Izadnegahdar M, Mackay M, Lee MK, Sedlak TL, Gao M, Bairey Merz CN, et al. Sex and ethnic differences in outcomes of acute coronary syndrome and stable angina patients with obstructive coronary artery disease. Circulation: Cardiovascular Quality and Outcomes. 2016;9(2_suppl_1):S26-S35.

12. Reis SE, Holubkov R, Smith AJC, Kelsey SF, Sharaf BL, Reichek N, et al. Coronary microvascular dysfunction is highly prevalent in women with chest pain in the absence of coronary artery disease: Results from the NHLBI WISE study. American Heart Journal. 2001;141(5):735-41.

13. Gulati M, Cooper-DeHoff RM, McClure C, Johnson BD, Shaw LJ, Handberg EM, et al. Adverse cardiovascular outcomes in women with nonobstructive coronary artery disease: a report from the Women's Ischemia Syndrome Evaluation Study and the St James Women Take Heart Project. Archives of internal medicine. 2009;169(9):843-50.

14. Rutten F, Bohnen A, Schreuder B, Pupping M, Bouma M. NHG-Standaard Stabiele angina pectoris. NHG-Standaarden 2009: Springer; 2009. p. 46-66.

15. Lam CS, Arnott C, Beale AL, Chandramouli C, Hilfiker-Kleiner D, Kaye DM, et al. Sex differences in heart failure. European Heart Journal. 2019;40(47):3859-68c. 

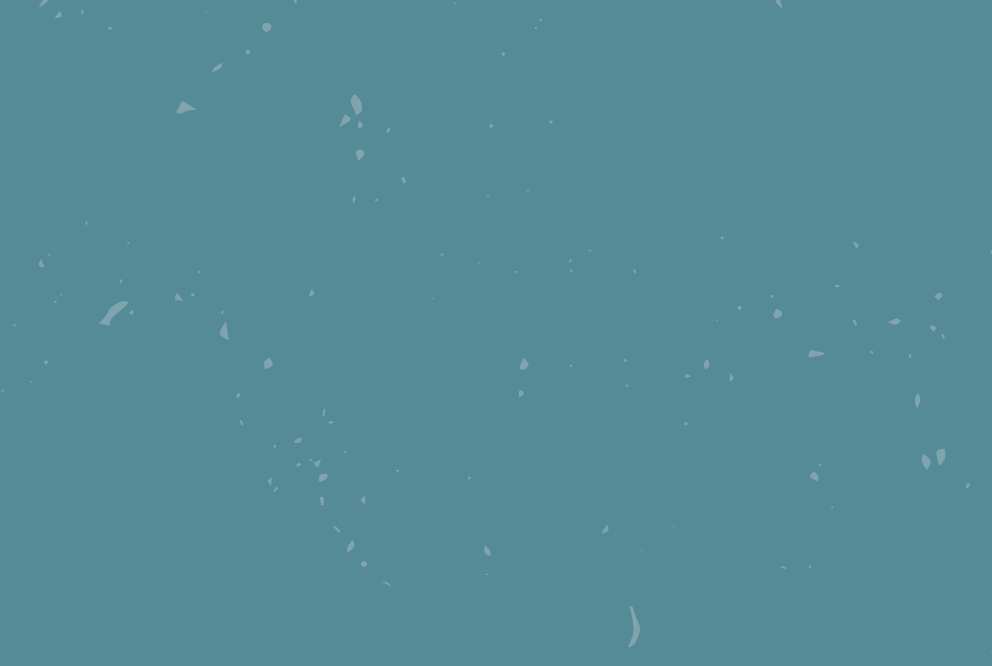

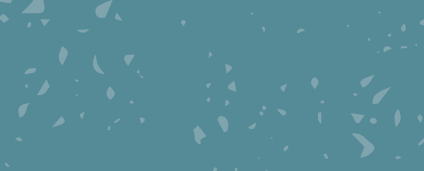




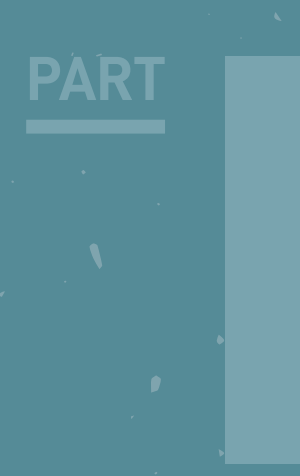

Symptoms 


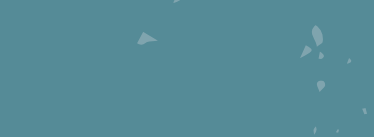

$\because \because \because \because \therefore \because \cdots$

$\quad \therefore \%$

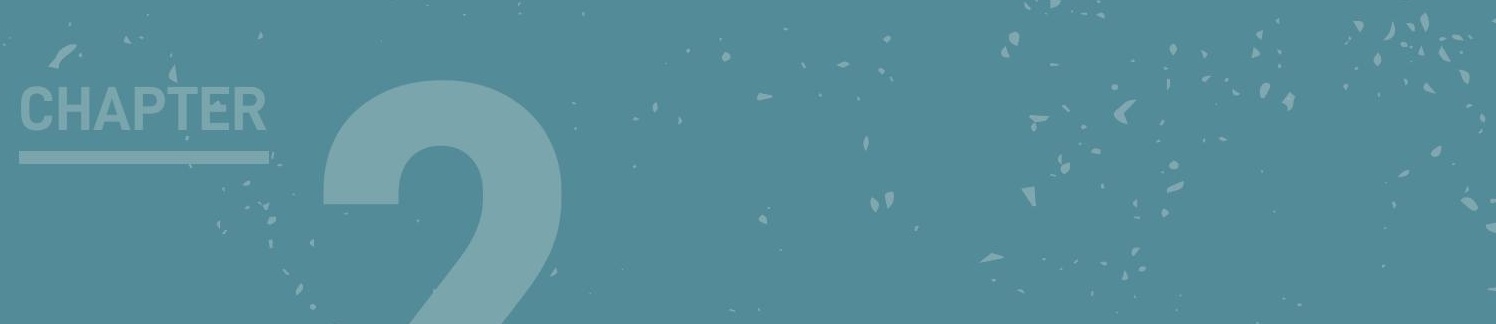
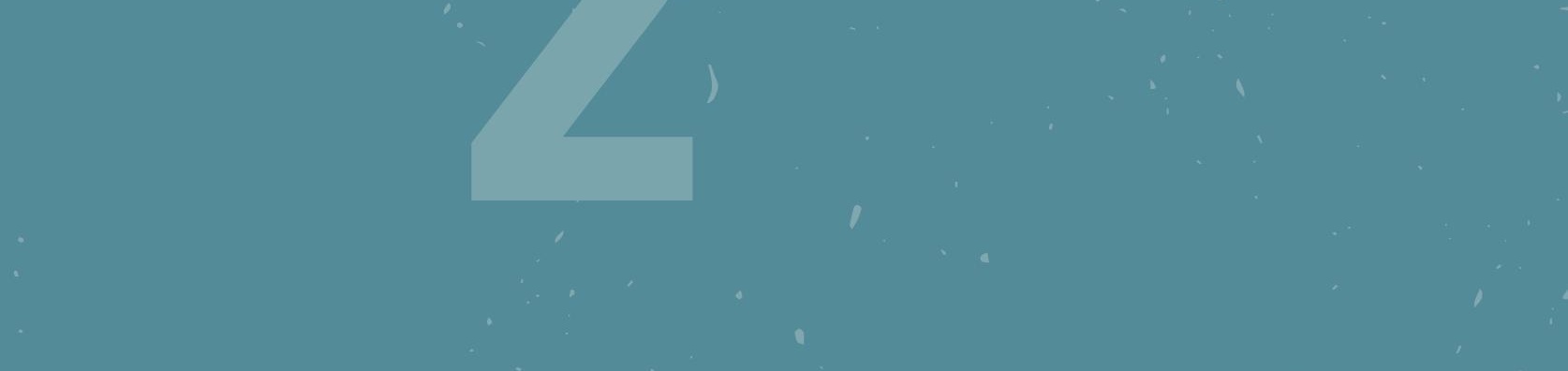

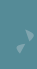

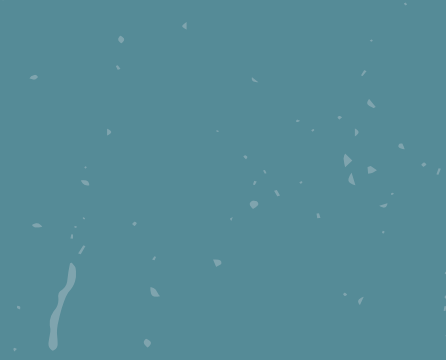

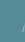




\section{Coronary artery disease prediction in women and men using chest pain characteristics and risk factors: an observational study in outpatient clinics}

BMJ Open 2020

Groepenhoff F, Eikendal ALM, Onland-Moret NC, Bots SH, Menken FR, Tulevski II, Somsen GA, Hofstra L*, den Ruijter HM* 


\section{ABSTRACT}

To assess the diagnostic value of non-acute chest pain characteristics for coronary artery disease in women and men referred to outpatient cardiology clinics, we performed an observational study at outpatient cardiology centers of the Netherlands. The study population consisted of 1028 non-acute chest pain patients (505 women). Twenty-four women (5\%) and seventy-five men (15\%) were diagnosed with coronary artery disease by invasive coronary angiography or computed tomographic angiography during regular care follow-up. Elastic net regression was performed to assess which chest pain characteristics and risk factors were of diagnostic value. The overall model selected age, provocation by temperature or stress, relief at rest and functional class as determinants and was accurate in both sexes (area under the curve (AUC) of 0.76 (95\% Cl: 0.68-0.85) in women and 0.83 (95\% Cl: 0.78-0.88) in men). Both sex-specific models selected age, pressuring nature, radiation, duration, frequency, progress, provocation and relief at rest as determinants. The female model additionally selected dyspnea, body mass index, hypertension and smoking while the male model additionally selected functional class and diabetes. The sex-specific models performed better than the overall model, but more so in women (AUC: 0.89, 95\% Cl: 0.81-0.96) than in men (AUC: $0.84,95 \% \mathrm{Cl}: 0.73-0.90$ ). In both sexes, the diagnostic value of non-acute chest pain characteristics and risk factors for coronary artery disease was high. Provocation, relief at rest and functional class of chest pain were the most powerful diagnostic predictors in both women and men. When stratified by sex the performance of the model improved, mostly in women. 


\section{INTRODUCTION}

Although women suffering from a myocardial infarction are reported to present with more atypical symptoms than men $(1,2)$, chest pain remains a major common symptom of coronary artery disease in both women and men (3). The relation between chest pain characteristics has been studied quite extensively in acute chest pain patients (1-9), yet not comprehensively in non-acute chest pain patients presenting at outpatient clinics.

Currently, approximately $1 \%$ of all patients consulting the general practitioner has chest pain complaints as main reason for their visit (10). However, only $8-12 \%$ of these patients actually have ischemic heart disease $(10,11)$. Forty percent of the patients with chest pain are referred to a cardiologist because of the fear of missing the potentially life-threatening diagnosis of coronary artery disease and because of difficulties in estimating the probability of coronary artery disease in those patients (12). In the Netherlands, this results in over 250,000 patients evaluated by a cardiologist for stable angina each year (13). Furthermore, additional (non-)invasive diagnostic tests are performed based on the cardiologists' estimation of the probability of coronary artery disease (14). For structural evaluation of the coronary arteries, cardiac computed tomography (calcium score with or without angiography) or invasive coronary angiography is used. Interestingly, in patients undergoing coronary angiography, no obstructive coronary artery disease is found twice as often in women as in men $(15,16)$. Additionally, approximately $50 \%$ of cardiac computed tomography investigations show a zero calcium score (17).

The high prevalence of normal imaging results raises the question whether the presence of coronary artery disease could be estimated with more certainty to prevent unnecessary imaging. In addition, differences in outcome and clinical presentation between women and men may suggest this estimation should take sex-specific symptoms and risk factors into account. According to the European and Dutch guidelines, chest pain characteristics are an important part of the diagnostic workup since these are considered helpful in estimating the probability of coronary artery disease $(14,18)$. However, their exact diagnostic value remains unclear $(9,19)$. Moreover, even though outpatient clinics evaluate the largest proportion of chest pain patients, most information on diagnostic value of signs and symptoms is derived from hospitals. As such, little is known on the diagnostic value of chest pain characteristics for coronary artery disease in men and women visiting outpatient cardiology clinics with non-acute chest pain. Therefore, we evaluated the diagnostic value of non-acute chest pain characteristics in differentiating coronary artery disease from no coronary artery disease patients in women and men presenting to outpatient cardiology clinics. 


\section{METHODS}

\section{Study design and population}

We retrospectively collected individual patient data records from Dutch outpatient cardiology clinics (Cardiology Centers of the Netherlands). Records contained baseline data from the electronic health records (CardioPortal ${ }^{\mathrm{TM}}$, Cardiology Centers of the Netherlands proprietary electronic health records). Ethical approval for this study was obtained from the Medical Research Ethics Committee of the University Medical Centre Utrecht and they waived the necessity for informed consent because research within the Cardiology Centers of the Netherlands database does not fall under the Dutch Medical Research Involving Human Subjects Act (WMO) (20). Patients visited one of the thirteen Cardiology Centers of the Netherlands locations between April 2007 and February 2018. We included data from patients referred by their general practitioner because of non-acute chest pain of whom chest pain characteristics were registered in a standardized way using a specified questionnaire. Standard cardiovascular workup for chest pain patients consisted of an intake by a nurse who collected information on cardiovascular risk factors (age, sex, history of smoking, history of cardiovascular disease, presence of diabetes mellitus, hypertension, dyslipidemia, body mass index (BMI) and signs of heart failure) and ischemia detection by exercise electrocardiogram, followed by evaluation by the cardiologist. All data were stored in a systematic manner. Patients were referred for cardiac computed tomography angiography or invasive coronary angiography only if the cardiologist estimated an intermediate or high probability of coronary artery disease (14). If referred for computed tomography, first a non-contrast enhanced cardiac computed tomography was performed to quantify the Agatston coronary artery calcium score (21). Additional computed tomography angiography was only performed in case of a positive calcium score. The results of these procedures were reported in the electronic health records.

\section{Chest pain characteristics}

Data records comprising the cardiologist's documented detailed and standardized description of non-acute chest pain characteristics $(n=1028)$ were retrieved from the electronic health records. These contained aspect, localization, radiation, onset, duration, frequency, progress, provoking and relieving factors and attendant symptoms. The functional degree of chest pain was scored by the cardiologist using the New York Heart Association classification (22). Based on prior studies, documented characteristics were reclassified into binary or categorical characteristics for analysis (18). This resulted in categorical characteristics for nature, dynamics (radiation, onset, duration, frequency, progress), co-existing symptoms (vagal complaints and dyspnea), provocation during exercise, emotional stress, cold or warmth, and relief at rest. 


\section{Definition of coronary artery disease}

The diagnosis of coronary artery disease was based on available imaging reports or, if no imaging was performed, on the cardiologist's estimation of the probability of coronary artery disease. Patients not referred for imaging, because of an estimated low probability of coronary artery disease by the cardiologist after standard cardiovascular workup, were considered to have no coronary artery disease. In patients referred for imaging, a significant stenosis (>50\%) was considered to indicate coronary artery disease (23). If the significance of a stenosis was not documented, a coronary artery calcium score above 160 was used as a surrogate marker for relevant coronary artery disease (21). Of 238 patients referred for imaging, 28 (12\%) were excluded from analysis due to missing imaging data.

\section{Statistical analysis}

Baseline characteristics and chest pain characteristics were stratified by sex and presented as mean \pm standard deviation (SD) for continuous variables and numbers (with \%) for categorical variables. The presence or absence of any cardiovascular history (e.g. previous coronary artery disease, cerebrovascular disease and cardiovascular intervention) was combined into one categorical variable for history of cardiovascular disease. Missing values for cardiovascular risk factors were complemented based on medication use if possible. Missing variables were imputed using the hot.deck function from the R package hot.deck (24). Information on the number (percentage) of missing variables is listed in Supplemental Appendix 1.

First, we tested which chest pain characteristics distinguished coronary artery disease from no coronary artery disease cases using a t-test for continuous variables and a $X^{2}$ test for categorical variables. Second, to build the most optimal model to discriminate between coronary artery disease and no coronary artery disease, elasticnet penalized logistic regression was performed. Elastic net was chosen as this is a dimensionality reduction method that can select important predictors in situations where there are a lot of predictors and a relatively modest number of events (25). As predictors both cardiovascular risk factors and chest pain characteristics were included. In total, 18 possible predictors were included in the multivariable elastic net analysis: 7 known cardiovascular risk factors (age, BMI, hypertension, dyslipidemia, diabetes mellitus, smoking status, cardiovascular history) and 11 chest pain characteristics (pressuring nature, radiation, onset, duration, frequency, progress, provocation during stress or extreme temperature, relief at rest, accompanying complaints, dyspnea, New York Heart Association class). We built the elastic net model with the R package glmnet (26) by using $66 \%$ of the data defined as training set and $33 \%$ as testing set (randomly selected). The optimal penalization proportion $\alpha$ was 
determined. The tuning parameter $\lambda$ was determined by choosing the largest $\lambda$ one standard deviation away from the $\lambda$ resulting in the minimal deviance of the model (minimizer $\lambda$ ). This tuning parameter would result in a sparse model. A sex-specific model for both women and men was fitted to evaluate possible sex differences in selected or relevance of variables as a subgroup analysis. Due to the decrease in power in the sex-specific elastic net regression, we determined the tuning parameter $\lambda$ as the minimizer $\lambda$ resulting in a less sparse model with which we minimized the deviance and were able to include more variables in the model.

Receiver operating characteristic (ROC) curves were constructed and areas under the curve (AUC) were calculated to estimate the performance of the model for women and men separately using the R package ROCR (27). $\mathrm{P}_{2 \text { sided }}<0.05$ was considered statistically significant. All statistical analyses were performed using RStudio, version 3.5.2 (www.r-project.org ) (28). Drs. Groepenhoff had full access to all the data in the study and took responsibility for the integrity of the data and the accuracy of the statistical analysis.

\section{RESULTS}

\section{Baseline characteristics}

Baseline characteristics of the study population are shown in Table 1. The total study population comprised 1028 patients of which 505 (49\%) were women. On average, women were two years older than men (58 years vs. 56 years, $p=0.009$ ). In women, a history of cardiovascular disease ( $8.3 \%$ in women vs. $13.8 \%$ in men, $p=0.007)$ and antihypertensive medication use ( $66 \%$ vs. $73 \%, p=0.04$ ) were less common than in men. The prevalence of all other cardiovascular risk factors was similar for women and men (Table 1).

After their visit to the cardiology clinic, 238 patients ( $23 \%$ of all patients, $44 \%$ women) were referred for cardiac computed tomography (angiography) or invasive coronary angiography. Of these 238 referred patients, 99 were diagnosed with coronary artery disease ( $42 \%$ of all patients, $24 \%$ women) (Figure 1). Detailed baseline characteristics of patients with coronary artery disease compared with patients without coronary artery disease are listed in Supplemental Appendix 2.

\section{Discriminating chest pain characteristics for CAD}

Table 2 shows the prevalence of chest pain characteristics for women and men stratified by the presence of coronary artery disease. In both women and men daily 
occurring and short-lasting chest pain were more prevalent in the presence of coronary artery disease as compared with no coronary artery disease. In women with coronary artery disease, daily occurring chest pain was prevalent in $63 \%$ versus $33 \%$ without coronary artery disease $(p=0.005)$. Daily occurring chest pain was common in $51 \%$ of men with coronary artery disease versus $28 \%$ of men without coronary artery disease $(p<0.001)$. Women with coronary artery disease reported short lasting chest pain in $96 \%$ as compared with $76 \%$ without coronary artery disease $(p<0.05)$. For short lasting chest pain, the prevalence was 93\% in men with coronary artery disease versus $72 \%$ of men with no coronary artery disease $(p<0.001)$. In addition, complaints were more often provoked by emotional or physical stress or extreme

Table 1. Characteristics of study population presenting at the outpatient cardiology clinics with chest pain complaints

\begin{tabular}{|c|c|c|c|c|}
\hline Demographic characteristics & Women & Men & & Total population \\
\hline $\mathbf{N}$ & 505 & 523 & p-value & 1028 \\
\hline Age (years) & $58(13)$ & $56(13)$ & 0.009 & $57(13)$ \\
\hline Current cigarette smoking status, yes $(n, \%)$ & $227(48)$ & $239(48)$ & 0.929 & $466(48)$ \\
\hline Diabetes mellitus, yes ( $n, \%)$ & $38(8)$ & $50(10)$ & 0.271 & $88(9)$ \\
\hline Hypertension, yes (n, \%) & $170(34)$ & $171(33)$ & 0.912 & $341(33)$ \\
\hline Dyslipidemia, yes (n, \%) & $83(17)$ & $101(20)$ & 0.233 & $184(18)$ \\
\hline Cardiovascular history, yes (n, \%) & $42(8)$ & $72(14)$ & 0.007 & $114(11)$ \\
\hline Antihypertensive medication, yes ( $n, \%)$ & $289(66)$ & $300(73)$ & 0.042 & $589(69)$ \\
\hline Statin use, yes $(n, \%)$ & $160(37)$ & $216(52)$ & $<0.001$ & $376(44)$ \\
\hline \multicolumn{5}{|l|}{ Anthropometric characteristics } \\
\hline $\mathrm{BMI}\left(\mathrm{kg} / \mathrm{m}^{2}\right)$ & $26(4.5)$ & $26(3.8)$ & 0.005 & $26(4.2)$ \\
\hline $\mathrm{SBP}(\mathrm{mmHg})$ & 139 (21.3) & 140 (18.5) & 0.347 & $140(20.0)$ \\
\hline $\mathrm{DBP}(\mathrm{mmHg})$ & $83(11.1)$ & $83(10.8)$ & 0.536 & $83(11.0)$ \\
\hline \multicolumn{5}{|l|}{ Chest pain specific characteristics } \\
\hline Pressuring nature, yes $(n, \%)$ & $411(81)$ & $402(77)$ & 0.088 & $813(79)$ \\
\hline Radiation, yes (n, \%) & $223(44)$ & $175(34)$ & 0.001 & $398(39)$ \\
\hline Acute onset, yes (n, \%) & $298(59)$ & $309(59)$ & 1.000 & $607(59)$ \\
\hline Short duration, yes (n, \%) & $391(77)$ & $394(75)$ & 0.474 & $785(76)$ \\
\hline Daily frequency, yes (n, \%) & $173(34)$ & $169(32)$ & 0.552 & $342(33)$ \\
\hline Progressive complaints, yes $(n, \%)$ & $141(28)$ & $147(28)$ & 1.000 & $288(28)$ \\
\hline $\begin{array}{l}\text { Provocation of complaints by stress/temp, } \\
\text { yes }(n, \%)\end{array}$ & $234(46)$ & $274(52)$ & 0.060 & $508(49)$ \\
\hline Relief at rest, yes (n, \%) & $214(42)$ & $243(47)$ & 0.209 & $457(45)$ \\
\hline Vegetative symptoms, yes (n, \%) & $81(16)$ & $62(12)$ & 0.065 & $143(14)$ \\
\hline Dyspnea, yes (n, \%) & 78 (15) & $61(12)$ & 0.093 & $139(14)$ \\
\hline NYHA class II or higher, yes (n, \%) & 166 (33) & $171(33)$ & 1.000 & $337(33)$ \\
\hline
\end{tabular}

BMI, body mass index; SBP, systolic blood pressure; DBP, diastolic blood pressure; NYHA, New York Heart Association 
temperature and alleviated in rest in patients with coronary artery disease as compared with patients without coronary artery disease. Provocation was prevalent in women in $75 \%$ of coronary artery disease cases versus $42 \%$ of no coronary artery disease cases $(p=0.003)$, and in $88 \%$ of men with coronary artery disease as compared with $44 \%$ of men without coronary artery disease $(p<0.001)$. Relief of chest pain complaints at rest was common in $75 \%$ in women with coronary artery disease versus $39 \%$ in women without coronary artery disease $(p=0.001)$. In men, complaints alleviated at rest in $83 \%$ with coronary artery disease and in $37 \%$ of men without coronary artery disease $(p<0.001)$. The functional class of chest pain was higher in

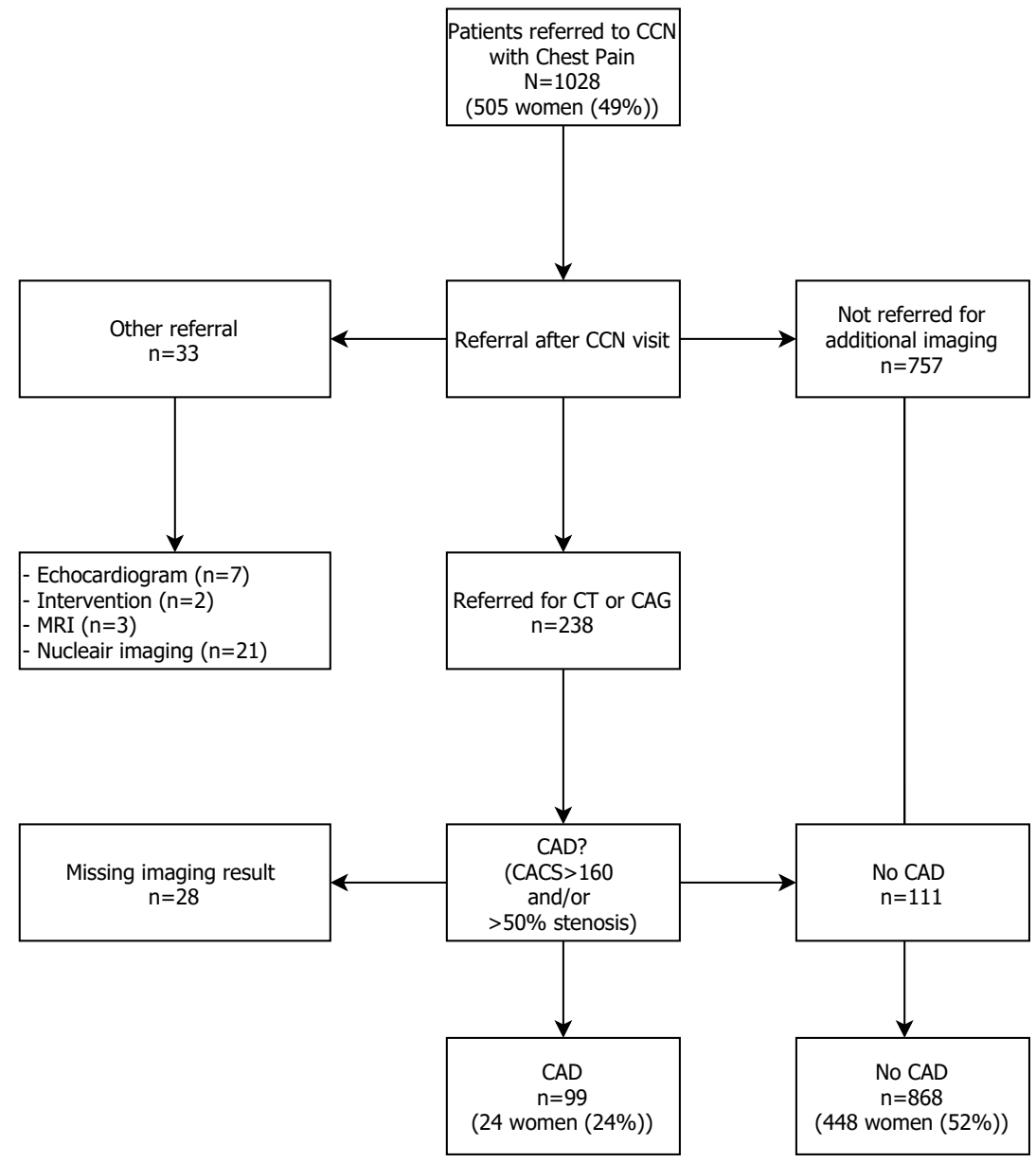

Figure 1. Overview of study population

$\mathrm{CCN}$, Cardiology Centers of the Netherlands; MRI, magnetic resonance imaging; CT, computed tomography; CAG, coronary angiography, CAD, coronary artery disease 
Table 2. Relation between chest pain characteristics and CAD

\begin{tabular}{|c|c|c|c|c|c|c|}
\hline & Women & & & Men & & \\
\hline Characteristic & $\begin{array}{l}\text { CAD } \\
(n=24)\end{array}$ & $\begin{array}{l}\text { No CAD } \\
(n=448)\end{array}$ & p-value & $\begin{array}{l}\text { CAD } \\
(n=75)\end{array}$ & $\begin{array}{l}\text { No CAD } \\
(n=420)\end{array}$ & $p$-value \\
\hline Pressing nature, yes ( $n, \%)$ & $22(91.7)$ & $361(80.6)$ & 0.278 & $72(96.0)$ & $303(72.1)$ & $<0.001$ \\
\hline Radiation, yes (n, \%) & $8(33.3)$ & $198(44.2)$ & 0.404 & $32(42.7)$ & $129(30.7)$ & 0.057 \\
\hline Acute onset, yes (n, \%) & $13(54.2)$ & $270(60.3)$ & 0.704 & $37(49.3)$ & $264(62.9)$ & 0.037 \\
\hline Short duration, yes (n, \%) & $23(95.8)$ & $341(76.1)$ & 0.046 & $70(93.3)$ & $301(71.7)$ & $<0.001$ \\
\hline Daily frequency, yes (n, \%) & $15(62.5)$ & $146(32.6)$ & 0.005 & $38(50.7)$ & 117 (27.9) & $<0.001$ \\
\hline Progressive, yes (n, \%) & $10(41.7)$ & $118(26.3)$ & 0.159 & $40(53.3)$ & $94(22.4)$ & $<0.001$ \\
\hline Provocation, yes (n, \%) & $18(75.0)$ & $190(42.4)$ & 0.003 & $66(88.0)$ & $184(43.8)$ & $<0.001$ \\
\hline Relief at rest, yes (n, \%) & $18(75.0)$ & $173(38.6)$ & 0.001 & $62(82.7)$ & $155(36.9)$ & $<0.001$ \\
\hline Vegetative symptoms, yes ( $n, \%)$ & $2(8.3)$ & $71(15.8)$ & 0.483 & $7(9.3)$ & $51(12.1)$ & 0.616 \\
\hline Dyspnea, yes (n, \%) & $6(25.0)$ & $66(14.7)$ & 0.284 & $15(20.0)$ & $39(9.3)$ & 0.011 \\
\hline NYHA class II or higher, yes ( $n, \%)$ & $13(54.2)$ & $135(30.1)$ & 0.025 & $57(76.0)$ & $97(23.1)$ & $<0.001$ \\
\hline
\end{tabular}

NYHA; New York Heart Association

coronary artery disease than in no coronary artery disease cases in both women and men: NYHA class II or higher in $54 \%$ of women and $76 \%$ of men with coronary artery disease as compared with $30 \%$ of women $(p=0.025)$ and $23 \%$ of men $(p<0.001)$ without coronary artery disease. In men, but not in women, pressuring nature $(96 \%$ vs. $72 \%, p<0.01)$, non-acute onset ( $51 \%$ vs. $37 \%, p=0.04$ ) progressive complaints $(53 \%$ vs. $22 \%, p<0.001)$ accompanied by dyspnea ( $20 \%$ vs. $9 \%, p<0.01)$ were discriminating coronary artery disease cases from those without coronary artery disease.

\section{Multivariable analysis by elastic net penalized regression}

In our elastic net analysis including both women and men the optimal model retained 4 out of 18 predictors with non-zero coefficients $(\alpha=0.68 ; \lambda=1$ SD from the minimizer $\lambda)$; age, provocation during stress or extreme temperature, relief at rest and NYHA class. The model distinguished women and men with coronary artery disease from those without coronary artery disease with an AUC of 0.82 ( $95 \% \mathrm{Cl}: 0.75-0.88)$. The AUC was 0.76 for women ( $95 \% \mathrm{Cl}: 0.68-0.85)$ and 0.83 (95\% $\mathrm{Cl}: 0.78-0.88)$ for men.

When we analyzed women separately, there were no predictors in the model. Therefore, we decided to use $\lambda=$ the minimizer $\lambda$ for the sex-specific analyses. In men, 10 clinical symptoms and risk factors with non-zero coefficients were retained in the model ( $\alpha=0.70, \lambda=$ the minimizer $\lambda$; age, pressuring nature, radiation, duration, frequency, progress, provocation during stress or extreme temperature, relief at rest, NYHA class and diabetes mellitus). The model achieved an AUC of 0.84 (95\% Cl: $0.73-$ 
0.90 ) in men (Table 3). In women, this elastic net regression resulted in a model with 12 clinical symptoms and risk factors with non-zero coefficients ( $\alpha=0.51, \lambda=$ the minimizer $\lambda$; age, pressuring nature, radiation, duration, frequency, progress, provocation during stress or extreme temperature, relief at rest, dyspnea, BMI, hypertension, smoking status). The model achieved an AUC of 0.89 (95\% Cl: $0.81-$ 0.96).

As described above, the overall model including both sexes achieved an AUC of 0.76 (95\% Cl: 0.68-0.85) in women and 0.83 (95\% Cl: 0.78-0.88) in men. Comparison of the performance of the overall model to that of the sex-specific models showed an improved performance in women (AUC of 0.89 (95\% Cl: $0.81-0.96)$ ) and only minimal improvement in men (AUC of 0.83 (95\% Cl: 0.73-0.90)).

Table 3. Sex-stratified elastic-net results of clinical characteristics and chest pain specific characteristics for CAD

\begin{tabular}{lll}
\hline & Women & Men \\
\cline { 2 - 3 } & Penalized coefficient & Penalized coefficient \\
\hline Age & 0.0208 & 0.0250 \\
Pressuring nature & 0.0336 & 0.5203 \\
Radiation & -0.0452 & 0.0252 \\
Duration & 0.1245 & 0.1475 \\
Frequency & 0.0868 & 0.1364 \\
Progressive complaints & 0.1168 & 0.0226 \\
Provocative complaints & 0.2205 & 0.5002 \\
Relief at rest & 0.3624 & 0.5569 \\
NYHA class & & 1.0619 \\
History of Diabetes & & 0.4927 \\
Dyspnea & 0.0118 & \\
Body mass index & -0.0169 & \\
History of Hypertension & 0.0467 & $\mathbf{0 . 8 4}(\mathbf{0 . 7 3 - 0 . 9 0 )}$ \\
Smoking status & -0.0708 & \\
\hline AUC (95\% Cl) & $\mathbf{0 . 8 9 ( 0 . 8 1 - 0 . 9 6 )}$ & \\
\hline
\end{tabular}

NYHA, New York Heart Association; AUC, area under the operator curve 


\section{DISCUSSION}

This study shows that characteristics of non-acute chest pain are useful in identification of coronary artery disease in both women and men referred to outpatient cardiology clinics. In fact, their diagnostic value appears to be reasonably high. Additionally, if presenting with non-acute chest pain, women and men with coronary artery disease present quite similar with regard to chest pain characteristics. However, small differences between women and men were observed with respect to the selected characteristics in the sex-specific models and their diagnostic performance. In women dyspnea, body mass index, hypertension and smoking were stronger predictors of the presence of coronary artery disease as compared with men. In men, functional class and history of diabetes were more important than in women. The performance of chest pain characteristics in diagnosing coronary artery disease improved when stratified by sex. This improvement was mostly seen in women in whom a separate model might be more appropriate than a model using data derived from both sexes.

Our analysis is based on routine care data of patients presenting at the outpatient cardiology clinic undergoing extensive standardized cardiovascular workup. This provided us with a unique representative sample of the general population who present to outpatient cardiology clinics with non-acute chest pain, without any selection bias that may occur in cohort studies in whom many patients may not participate due to their poor quality of life (29). Secondly, our study contained an almost equal number of women and men, which enabled us to study both women and men with equal power.

In the primary care setting, Bösner et al. (19) assessed sex differences in clinical characteristics of chest pain. The researchers observed some chest pain characteristics, for example pain worsening with exercise, to be equally relevant for men and women. Yet, in women, prolonged duration of chest pain was positively associated with coronary artery disease, whereas in men shorter duration showed a positive association with coronary artery disease. These chest pain characteristics are different from those reported in our study, namely dyspnea in women and functional class in men. This difference might be due to the dissimilarity in domain as the women and men presented at outpatient cardiology clinics were already referred by their general practitioner. In an additional study, the researchers observed a combination of clinical characteristics and symptoms in patients presenting with chest pain to be helpful in diagnosing coronary heart disease (30). Their results support the high diagnostic value of chest pain characteristics in differentiating coronary artery disease from no coronary artery disease patients that we observed in both sexes as well as the small 
sex differences in associated chest pain characteristics with coronary artery disease. This suggests that using a sex-stratified method may improve the diagnostic approach within these patients.

Several studies performed at the emergency department showed a similar high diagnostic value of clinical symptoms and cardiovascular risk factors for coronary artery disease in patients presenting with chest pain $(6,7,9)$. They showed women and men to present quite similar if presenting with chest pain (7). Moreover, Ferry et al. (6) reported that in patients diagnosed with myocardial infarction, typical symptoms were more prevalent and had a higher predictive value in women than in men. In line with our findings and the aforementioned findings in primary care, van der Meer et al. (9) and Gimenez et al. (7) also only found small sex differences in chest pain characteristics associated with coronary artery disease.

Interestingly, even though pressuring nature of chest pain is often seen as one of the most typical and suspect characteristics in the evaluation of chest pain, this characteristic was not retained in the overall elastic net model. This is similar to a previous report (9). Albeit pressuring nature was differentiating between coronary artery disease and no coronary artery disease in both men and women in our univariable analysis, it shrunk to zero in our most optimal elastic-net model. This could be a result of the correlation of pressuring nature with other chest pain characteristics retained in the model that had a stronger association with coronary artery disease, as the elastic-net analysis is designed to create a model with a high diagnostic accuracy with fewest variables. Radiation was not significantly different between women and men with and without coronary artery disease in our univariable analyses, as was also shown in earlier research (30).

Our study shows that chest pain characteristics have diagnostic value in women and men presenting with non-acute chest pain at outpatient cardiology clinics. Our results were comparable to the acute setting at the emergency department, and also to the primary care setting. This implies that these easy to obtain chest pain characteristics are equally predictive in high $(7,9)$ as well as in low risk $(19)$ populations. These insights obtained from different clinical settings enable both the general practitioner and the cardiologist to improve identification of women and men at risk for coronary artery disease. This knowledge, when brought to practice, may lower the currently rising costs of healthcare due to unnecessary cardiac workup and imaging. Besides reducing healthcare costs, it may improve patient care since less imaging reduces the amount of radiation used and the burden of an unnecessary examination. 
Within the outpatient clinics assessed in this study, CT or CAG is the preferred method for the evaluation of coronary artery disease. However, non-invasive stress imaging is also recommended and frequently performed for the evaluation of coronary artery disease in patients presenting with chest pain in current clinical care (14). To improve the diagnostic approach in patients presenting with chest pain beyond the evaluation of anatomical coronary artery disease by CT or CAG, it would be interesting to evaluate whether clinical characteristics could add to the evaluation of ischemia in patients presenting with chest pain as well and could be useful to improve patient selection for non-invasive stress imaging.

\section{LIMITATIONS}

As our study was based on data of electronic health records, data was only available if documented as part of routine clinical care. This may have resulted in some limitations of this study. First, chest pain characteristics were collected as part of the regular diagnostic workup using a specific questionnaire and not available for every patient presenting with chest pain. Hence, missing data with respect to the chest pain characteristics may have caused bias. Cardiologists may have only recorded chest pain characteristics when chest pain was the prevailing complaint. As such, our conclusion may not be applicable to patients who present with mild dyspnea and chest discomfort. Also, some chest pain characteristics (e.g., vagal symptoms) were not reported enough to be studied. Furthermore, not all patients in our study were referred for diagnostic imaging, and we have assumed that these non-referred women and men were free from coronary artery disease which may not have been the case. This may have resulted in misclassification of patients (4). This misclassification of coronary artery disease may have result in an underestimation of the diagnostic value of chest pain characteristics for coronary artery disease. As such, the diagnostic value of chest pain characteristics may in fact even be higher than reported in this study.

Finally, since our data was collected during clinical care and was not available for patients that were referred back to their general practitioner, we did not have access to long-term follow up information nor were we allowed to contact patients to obtain this information. As a result, our study was limited to diagnostic research evaluating the absence or presence of obstructive coronary artery disease as assessed by CT and/or CAG. Our data did not enable us to expand our research to the diagnostic value of chest pain specific characteristics for coronary microvascular disease or assessment of their prognostic value within patients presenting with chest pain with(out) coronary artery disease presenting to the outpatient clinics. Future studies 
are needed us to elucidate the prognostic value of chest pain specific characteristics for cardiac events within all patients presenting with chest pain.

\section{CONCLUSION}

In both men and women, the diagnostic value of non-acute chest pain characteristics and risk factors in differentiating coronary artery disease from no coronary artery disease was high. Provocation by temperature or stress, relief at rest and functional class of chest pain were most powerful in diagnosing coronary artery disease. Furthermore, when presenting with non-acute chest pain, women and men with coronary artery disease present quite similar with regard to chest pain characteristics. Nonetheless, our data does show small sex differences regarding chest pain characteristics and stratified by sex the performance of the model improved, mostly in women. These results might suggest the need for a sex-specific model in diagnosing coronary artery disease and a possibility to improve referral for cardiac imaging of women and men at the outpatient clinic with non-acute chest pain.

\section{Acknowledgements}

We thank all people involved from Cardiology Center Netherlands and specifically the team at Cardiology Center Utrecht for their assistance.

\section{Funding}

The first author (FG) is supported by funding from CVON 2017-22 ARGUS. 


\section{REFERENCES}

1. Čulić V, Eterović D, Mirić D, Silić NJAhj. Symptom presentation of acute myocardial infarction: influence of sex, age, and risk factors. 2002;144(6):1012-7.

2. Canto JG, Rogers WJ, Goldberg RJ, Peterson ED, Wenger NK, Vaccarino V, et al. Association of age and sex with myocardial infarction symptom presentation and in-hospital mortality. 2012;307(8):813-22.

3. Canto JG, Goldberg RJ, Hand MM, Bonow RO, Sopko G, Pepine CJ, et al. Symptom presentation of women with acute coronary syndromes: myth vs reality. Archives of internal medicine. 2007;167(22):2405-13.

4. de Torbal A, Boersma E, Kors JA, van Herpen G, Deckers JW, van der Kuip DA, et al. Incidence of recognized and unrecognized myocardial infarction in men and women aged 55 and older: the Rotterdam Study. 2006;27(6):72936.

5. DeVon HA, Zerwic JJ. Symptoms of acute coronary syndromes: are there gender differences? A review of the literature. Heart \& Lung: The Journal of Acute and Critical Care. 2002;31(4):235-45.

6. Ferry AV, Anand A, Strachan FE, Mooney L, Stewart SD, Marshall L, et al. Presenting symptoms in men and women diagnosed with myocardial infarction using sex-specific criteria. Journal of the American Heart Association. 2019;8(17):e012307.

7. Gimenez MR, Reiter M, Twerenbold R, Reichlin T, Wildi K, Haaf $P$, et al. Sex-specific chest pain characteristics in the early diagnosis of acute myocardial infarction. JAMA internal medicine. 2014;174(2):241-9.

8. Milner KA, Funk M, Richards S, Wilmes RM, Vaccarino V, Krumholz HM. Gender differences in symptom presentation associated with coronary heart disease. The American journal of cardiology. 1999;84(4):396-9.

9. Van Der Meer MG, Backus BE, Van Der Graaf Y, Cramer MJ, Appelman Y, Doevendans PA, et al. The diagnostic value of clinical symptoms in women and men presenting with chest pain at the emergency department, a prospective cohort study. PloS one. 2015;10(1):e0116431.

10. Bösner S, Becker A, Haasenritter J, Abu Hani M, Keller H, Sönnichsen AC, et al. Chest pain in primary care: epidemiology and pre-work-up probabilities. The European journal of general practice. 2009;15(3):141-6.

11. Nilsson S, Scheike M, Engblom D, Karlsson L-G, Mölstad S, Akerlind I, et al. Chest pain and ischaemic heart disease in primary care. Br J Gen Pract. 2003;53(490):378-82.

12. Hoorweg BB, Willemsen RT, Cleef LE, Boogaerts T, Buntinx F, Glatz JF, et al. Frequency of chest pain in primary care, diagnostic tests performed and final diagnoses. Heart. 2017:heartjnl-2016-310905.

13. Nederland Zorginstituut. Verbetersignalement Pijn op de borst (verdenking) stabiele angina pectoris. https://www.zorginstituutnederland.nl/publicaties/rapport/2018/01/31/zinnige-zorg-verbetersignalement-\%E2\%80\%98pijn-op-de-borst\%E2\%80\%992017. p. 80.

14. Members TF, Montalescot G, Sechtem U, Achenbach S, Andreotti F, Arden C, et al. 2013 ESC guidelines on the management of stable coronary artery disease: the Task Force on the management of stable coronary artery disease of the European Society of Cardiology. European heart journal. 2013;34(38):2949-3003.

15. Shaw LJ, Merz CNB, Pepine CJ, Reis SE, Bittner V, Kip KE, et al. The economic burden of angina in women with suspected ischemic heart disease: results from the National Institutes of Health-National Heart, Lung, and Blood Institute-sponsored Women's Ischemia Syndrome Evaluation. Circulation. 2006;114(9):894-904.

16. Jespersen L, Hvelplund A, Abildstrøm SZ, Pedersen F, Galatius S, Madsen JK, et al. Stable angina pectoris with no obstructive coronary artery disease is associated with increased risks of major adverse cardiovascular events. European heart journal. 2011;33(6):734-44.

17. Mittal TK, Pottle A, Nicol E, Barbir M, Ariff B, Mirsadraee S, et al. Prevalence of obstructive coronary artery disease and prognosis in patients with stable symptoms and a zero-coronary calcium score. European Heart JournalCardiovascular Imaging. 2017;18(8):922-9.

18. Rutten F, Bohnen A, Schreuder B, Pupping M, Bouma M. NHG-Standaard Stabiele angina pectoris. NHG-Standaarden 2009: Springer; 2009. p. 46-66.

19. Bösner S, Haasenritter J, Hani MA, Keller H, Sönnichsen AC, Karatolios K, et al. Gender differences in presentation and diagnosis of chest pain in primary care. BMC family practice. 2009;10(1):79.

20. Gevers J. Medisch-wetenschappelijk onderzoek met mensen. Tijdschrift voor Gezondheidsrecht. 2001;25(1):17.

21. Arad Y, Spadaro LA, Goodman K, Newstein D, Guerci AD. Prediction of coronary events with electron beam computed tomography. Journal of the American College of Cardiology. 2000;36(4):1253-60.

22. Levin R, Dolgin M, Fox C, Gorlin R. The Criteria Committee of the New York Heart Association. inNomenclature and criteria for diagnosis of diseases of the heart and great vessels, Iww handbooks. 1994;9:253-6. 
23. Bruschke AV, Proudfit WL, Sones JR FM. Progress study of 590 consecutive nonsurgical cases of coronary disease followed 5-9 years: II. ventriculographic and other correlations. Circulation. 1973;47(6):1154-63.

24. Cranmer SJ, Gill J. We have to be discrete about this: A non-parametric imputation technique for missing categorical data. British Journal of Political Science. 2013;43(2):425-49.

25. Zou H, Hastie T. Regularization and variable selection via the elastic net. Journal of the royal statistical society: series B (statistical methodology). 2005;67(2):301-20.

26. Friedman J, Hastie T, Tibshirani R. Regularization paths for generalized linear models via coordinate descent. Journal of statistical software. 2010;33(1):1.

27. Sing T, Sander O, Beerenwinkel N, Lengauer T. ROCR: visualizing classifier performance in R. Bioinformatics. 2005;21(20):3940-1.

28. Team RC. R: A language and environment for statistical com-puting. R Foundation for Statistical Computing, Vienna, Austria URL https://www R-project org. 2017.

29. Gijsberts CM, den Ruijter HMJljoc. Non-response to questionnaires independently predicts mortality of coronary angiography patients. 2015;201:168-70.

30. Bösner S, Becker A, Hani MA, Keller H, Sönnichsen AC, Haasenritter J, et al. Accuracy of symptoms and signs for coronary heart disease assessed in primary care. Br J Gen Pract. 2010;60(575):e246-e57. 


\section{SUPPLEMENTARY MATERIAL}

Supplemental appendix 1. Percentage missing characteristics of study population presenting at the outpatient cardiology clinics with chest pain complaints

\begin{tabular}{|c|c|c|c|}
\hline Demographic characteristics & Women & Men & $\begin{array}{l}\text { Total } \\
\text { population }\end{array}$ \\
\hline $\mathrm{N}$ & 505 & 523 & 1028 \\
\hline Age & 0 & 0 & \\
\hline Current cigarette smoking status & 6.7 & 4.2 & 5.4 \\
\hline Diabetes mellitus & 0.2 & 1.1 & 0.7 \\
\hline Hypertension & 0.0 & 1.3 & 0.7 \\
\hline Dyslipidemia & 0.2 & 1.1 & 0.7 \\
\hline Cardiovascular history & 0 & 0 & 0 \\
\hline Antihypertensive medication & 13.5 & 21.2 & 17.4 \\
\hline Statin use & 13.5 & 21.2 & 17.4 \\
\hline \multicolumn{4}{|l|}{ Anthropometric characteristics } \\
\hline Body mass index & 1.8 & 3.3 & 2.5 \\
\hline Systolic blood pressure & 8.5 & 7.5 & 8.0 \\
\hline Diastolic blood pressure & 8.3 & 7.5 & 7.9 \\
\hline \multicolumn{4}{|l|}{ Chest pain specific characteristics } \\
\hline Pressuring nature & 0.0 & 0.0 & 0.0 \\
\hline Radiation & 0.0 & 0.0 & 0.0 \\
\hline Acute onset & 0.0 & 0.0 & 0.0 \\
\hline Short duration & 0.0 & 0.0 & 0.0 \\
\hline Daily frequency & 0.0 & 0.0 & 0.0 \\
\hline Progressive complaints & 0.0 & 0.0 & 0.0 \\
\hline Provocation of complaints by stress/temp & 0.0 & 0.0 & 0.0 \\
\hline Relief at rest & 0.0 & 0.0 & 0.0 \\
\hline Vegetative symptoms & 0.0 & 0.0 & 0.0 \\
\hline Dyspnea & 0.0 & 0.0 & 0.0 \\
\hline NYHA class & 0.0 & 0.0 & 0.0 \\
\hline
\end{tabular}


Supplemental appendix 2. Characteristics of study population stratified by Coronary Artery Disease (CAD), $n=967$ due to missing imaging results

\begin{tabular}{|c|c|c|c|c|}
\hline \multirow[t]{3}{*}{ Demographic characteristics } & \multicolumn{2}{|l|}{ Women } & \multicolumn{2}{|l|}{ Men } \\
\hline & No CAD & CAD & No CAD & CAD \\
\hline & 448 & 24 & 420 & 75 \\
\hline Age (years) & $57(12.8)$ & $68(7.0)$ & $54(13.5)$ & $63(10.0)$ \\
\hline Current cigarette smoking status, yes ( $n, \%)$ & $231(48)$ & $10(42)$ & $226(51)$ & $26(34)$ \\
\hline Diabetes mellitus, yes ( $n, \%)$ & $34(8)$ & $3(13)$ & $32(8)$ & $15(20)$ \\
\hline Hypertension, yes (n, \%) & $142(32)$ & $11(46)$ & $122(30)$ & 37 (49) \\
\hline Dyslipidemia, yes (n, \%) & 74 (17) & $5(21)$ & $72(17)$ & $20(27)$ \\
\hline Cardiovascular history, yes (n, \%) & $33(7)$ & $4(17)$ & $42(10)$ & $22(29)$ \\
\hline Antihypertensive medication, yes ( $n, \%)$ & $239(63)$ & $22(92)$ & $199(64)$ & $74(100)$ \\
\hline Statin use, yes (n, \%) & $125(33)$ & $17(71)$ & $133(43)$ & $63(85)$ \\
\hline \multicolumn{5}{|l|}{ Anthropometric characteristics } \\
\hline Body mass index $\left(\mathrm{kg} / \mathrm{m}^{2}\right)$ & $26(4.5)$ & $24(3.6)$ & $27(3.7)$ & $28(4.2)$ \\
\hline Systolic blood pressure $(\mathrm{mmHg})$ & $139(20.9)$ & $147(22.9)$ & $140(18.2)$ & $143(20.1)$ \\
\hline Diastolic blood pressure $(\mathrm{mmHg})$ & $83(10.85)$ & 78 (11.7) & $84(11.1)$ & $81(8.8)$ \\
\hline \multicolumn{5}{|l|}{ Chest pain specific characteristics } \\
\hline Pressing nature, yes ( $n, \%)$ & $303(72)$ & $72(96)$ & $361(81)$ & $22(92)$ \\
\hline Radiation, yes (n, \%) & $129(31)$ & $32(43)$ & $198(44)$ & $8(33)$ \\
\hline Acute onset, yes ( $n, \%)$ & $264(63)$ & $37(49)$ & $270(60)$ & $13(54)$ \\
\hline Short duration, yes ( $n, \%)$ & $301(72)$ & $70(93)$ & $341(76)$ & $23(96)$ \\
\hline Daily frequency, yes (n, \%) & $117(28)$ & $38(51)$ & $146(33)$ & $15(62)$ \\
\hline Progressive complaints, yes (n, \%) & $94(23)$ & $40(53)$ & $118(26)$ & $10(42)$ \\
\hline Provocation of complaints by stress/temp, yes (n, \%) & $184(44)$ & $66(88)$ & $190(42)$ & $18(75)$ \\
\hline Relief at rest, yes (n, \%) & $155(37)$ & $62(83)$ & $173(39)$ & $18(75)$ \\
\hline Vegetative symptoms, yes ( $n, \%)$ & $51(12)$ & $7(9)$ & $71(16)$ & $2(8)$ \\
\hline Dyspnea, yes (n, \%) & $39(9)$ & $15(20)$ & $66(15)$ & $6(25)$ \\
\hline NYHA class II or higher, yes ( $n, \%)$ & $97(23)$ & $57(76)$ & $135(30)$ & $13(54)$ \\
\hline
\end{tabular}




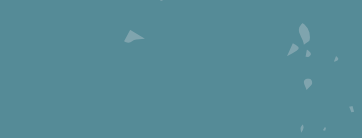

$\because \because \quad \because \therefore \quad \therefore$ CHAPTER
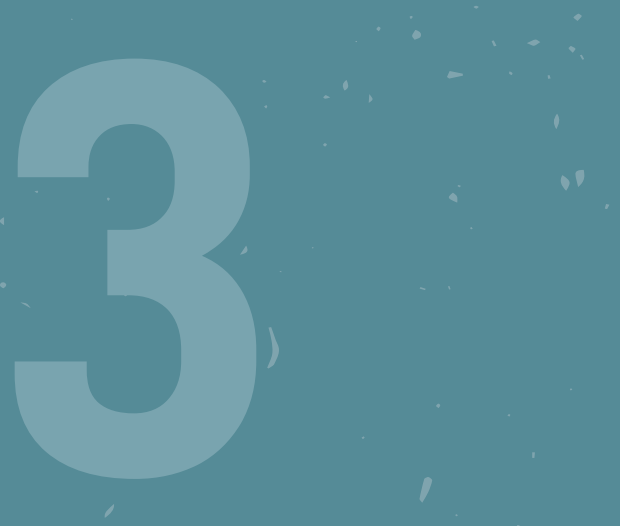

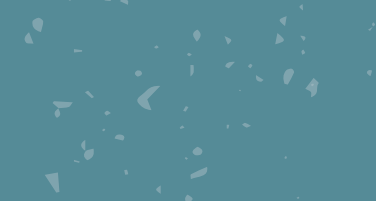

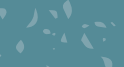
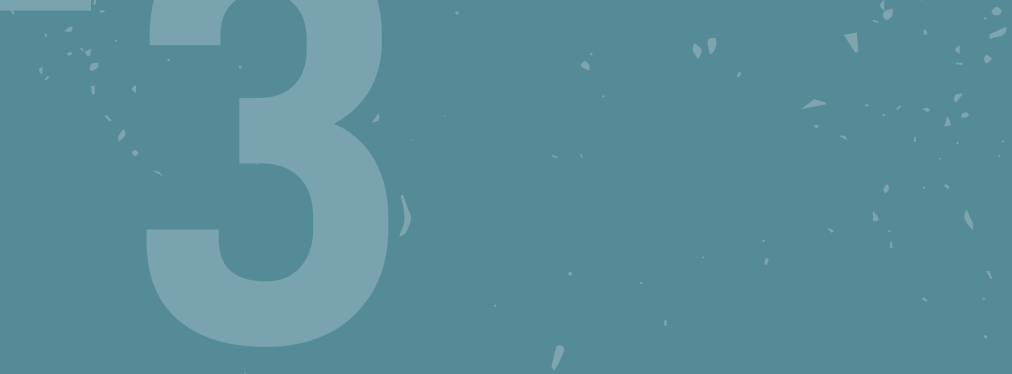

i.

a

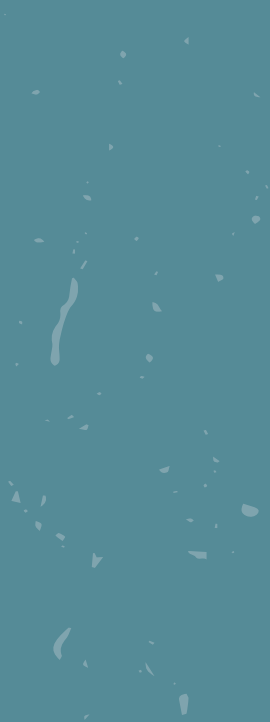

$\because$

,

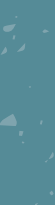




\section{NYHA class is strongly associated with mortality beyond heart failure in symptomatic women}

Published as research letter in EHJ - Quality of Care and Clinical Outcomes 2021

Groepenhoff F*, Siegersma KR*, Onland-Moret NC, Tulevski II, Hofstra L, Somsen GA, den Ruijter HM 
Cardiovascular disease remains the leading cause of mortality worldwide for women, which stresses the need for accurate risk prediction in women (1). The New York Heart Association (NYHA) functional classification of patients with cardiovascular disease is a commonly used clinical scale to estimate the general condition of a patient (2). It has been specifically designed in and used for (acute) heart failure patients and has shown to be a valid and easy obtainable measure to predict mortality in these patients (3). Nowadays, use of this clinical scale is not solely limited to heart failure patients but widely used by cardiologists to grade severity of several complaints, i.e. chest pain, dyspnea and fatigue, and classify the condition of an individual (4). However, most validation studies have been performed in men with acute heart failure and recent studies in more heterogeneous populations showed that the predictive value of NYHA class for mortality might differ between the sexes and patient domains $(3,5)$.

As the diagnosis is challenging, a variety of clinical scales are used and tests are performed to assess cardiovascular disease (risk). Yet, clinicians do not know which of these performs best in women, because originally diagnostic risk stratification tools were mainly developed in men. Since the NYHA classification scale is an easily obtainable non-invasive tool without any burden on the patient, it is often used in women at outpatient clinics. However, the value of NYHA class for prediction of mortality in this large and heterogeneous female population is unknown. Therefore, in women with a variety of symptoms, i.e. chest pain, dyspnea and fatigue, we specifically studied the association between NYHA class and mortality in a large population of patients presenting at outpatient cardiology clinics.

We extracted electronic health record data of thirteen outpatient clinics (Cardiology Centers of the Netherlands) for individuals that have visited between 2007 and 2018 with a documented NYHA class for chest pain, dyspnea or fatigue at their initial visit. Patients received a diagnostic workup, including NYHA class for chest pain, dyspnea or fatigue, blood tests, echocardiography, a stress and rest electrocardiogram, full anamnesis by a specialized nurse and a cardiologist' consult. Follow-up for mortality was performed by linkage to the population registry of Statistics Netherlands. We estimated survival functions using the Kaplan-Meier method. Cox proportional hazards regression analysis was used to study the association between NYHA class and mortality corrected for age. All analyses were performed per sex and stratified by primary complaint (i.e. chest pain, dyspnea and fatigue). All statistical analyses were performed in $\mathrm{R}$ (version 4.0.2). The Medical Research Ethics Committee of the UMCU waived the necessity for informed consent because research within the Cardiology Centers of the Netherlands database does not fall under the Dutch Medical 
Research Involving Human Subjects Act (Wet medisch-wetenschappelijk onderzoek met mensen, WMO).

Of the 9011 patients, 4782 (53\%) were female of whom 1450 presented with dyspnea, 2801 with chest pain and 531 with fatigue as primary complaint. NYHA class I, II and III-IV out of IV were respectively documented in 2196 (46\%), 2077 (43\%) and 509 (11\%) women (for male patients, this distribution was 2114 (50\%), 1688 (40\%) and $428(10 \%)$, respectively). Higher NYHA class at baseline was related to higher age. Other standard cardiovascular risk factors were similar between the NYHA classes (Figure 1, panel B). After eight years of follow up 354 (7\%) women and 415 (10\%) men died, of which 134 (38\%) and 150 (36\%), respectively, were classified as cardiovascular death.

In all women, regardless of primary complaint, survival analysis showed that increased NYHA class was positively associated with mortality, both for all-cause (Figure 1) and cardiovascular (data not shown) mortality. Multivariable analysis corrected for age confirmed that women with NYHA class III-IV have a higher risk of mortality as compared with women presenting with NYHA class I (all women: hazard ratio (HR) 3.9, 95\% confidence interval (Cl) 2.8-5.5, chest pain: $\mathrm{HR} 2.4,95 \% \mathrm{Cl} 1.3-4.6$, dyspnea: HR 2.6, 95\% Cl 1.5-4.6, fatigue: HR 2.5, 95\% Cl 1.0-6.0). Women suffering from complaints classified as NYHA class II showed a similar, but less evident, trend (all women: $\mathrm{HR} 1.7,95 \% \mathrm{Cl} 1.3-2.3$, chest pain: $\mathrm{HR} 1.4,95 \% \mathrm{Cl} 0.8-2.2$, dyspnea: HR 1.2 $95 \% \mathrm{Cl} 0.7-2.1$, fatigue: $\mathrm{HR} 0.9,95 \% \mathrm{Cl} 0.4-2.1)$. In all men, and in men presenting with dyspnea, chest pain or fatigue separately, a similar relationship between NYHA class and (cardiovascular) mortality was found (NYHA class III-IV vs. I in all men: HR 4.4, 95\% Cl 3.2-6.0, chest pain: HR 2.3, 95\% Cl 1.4-3.8, dyspnea: HR 4.1, 95\% Cl 2.47.8, fatigue: $\mathrm{HR} 7.9,95 \% \mathrm{Cl} 2.0-31.3$, data on cardiovascular mortality not shown). The association between NYHA class III-IV and mortality remained significant for both women and men after adjustment for SCORE (women: HR 7.8, 95\% Cl 4.9-12.2, men: HR 7.1, 95\% Cl 4.8-10.5).

Similar to men, in women presenting with chest pain, fatigue or dyspnea at outpatient cardiology clinics functional grading of their complaints using NYHA classification provides important information on their (cardiovascular) mortality risk. As NYHA class is an easily obtainable, non-invasive measure, treating physicians are able to quickly get an idea of the physical status and survival risk of the visiting female patient. We have shown that this is the case for patients with a wide range of complaints and not solely for heart failure patients. Therefore, women suffering from NYHA class III-IV complaints may warrant aggressive diagnostic and therapeutic workup. 
A

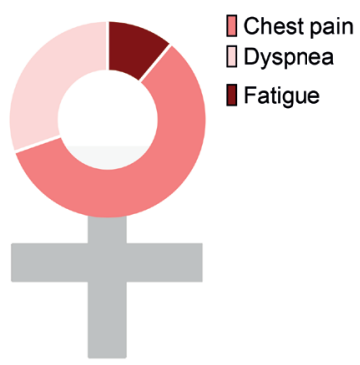

C

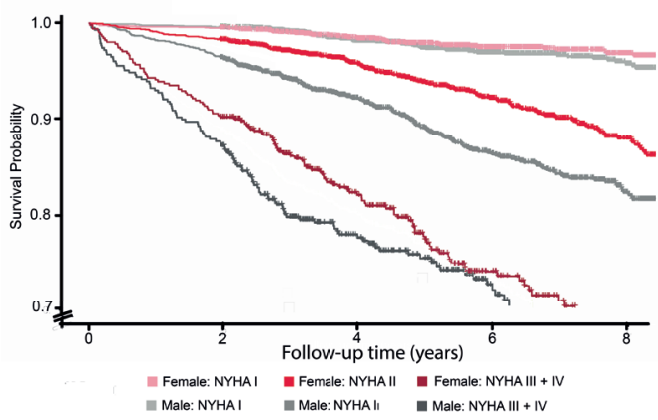

B
NYHA Class I NYHA Class II $\quad$ NYHA Class III+IV

\begin{tabular}{|lr|r|r|} 
& NYHA Class I & NYHA Class II & NYHA Class III+IV \\
& 2196 & 2077 & 509 \\
\hline Age (mean(SD)) & $55.6(13.3)$ & $63.7(12.8)$ & $69.8(12.4)$ \\
\hline BMI (mean(SD)) & $25.7(4.7)$ & $27.5(5.2)$ & $28.4(6.4)$ \\
\hline Current smoker(\%) & $782(39.1)$ & $758(39.2)$ & $182(38.6)$ \\
\hline No diabetes (\%) & $2087(95.3)$ & $1849(89.3)$ & $428(84.6)$ \\
\hline No hypertension (\%) & $1607(73.3)$ & $1225(59.2)$ & $253(49.9)$ \\
\hline No dyslipidaemia (\%) & $1912(87.2)$ & $1606(77.5)$ & $406(80.1)$ \\
\hline SCORE (mean(SD)) & $3(5.9)$ & $6(10.3)$ & $11(12.8)$
\end{tabular}

D

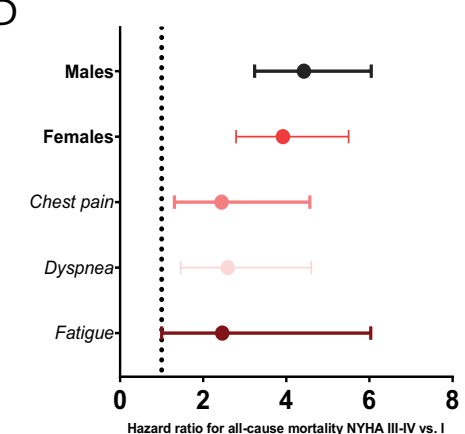

Figure 1. NYHA class in symptomatic women at the outpatient cardiology clinic

A. Prevalence of complaints in women. B. Baseline table of symptomatic women stratified by NYHA-class. C. Mortality during follow up according to NYHA class. D. Hazard ratio for all-cause mortality in all men, all women and in women stratified by complaint.

BMI, body mass index; SCORE, Systematic COronary Risk Evaluation; SD, standard deviation; NYHA, New York Heart Association Classification 


\section{REFERENCES}

1. Virani SS, Alonso A, Benjamin EJ, Bittencourt MS, Callaway CW, Carson AP, et al. Heart disease and stroke statistics-2020 update: a report from the American Heart Association. Circulation. 2020:E139-E596.

2. Dolgin M, NYHA Association Criteria Comittee. Nomenclature and criteria for diagnosis of diseases of the heart and great vessels: Boston: Little, Brown \& Co; 1994.

3. Kajimoto K, Sato N, Registry lotADHFS. Sex differences in New York Heart Association Functional Classification and survival in acute heart failure patients with preserved or reduced ejection fraction. Canadian Journal of Cardiology. 2020;36(1):30-6.

4. Yancy CW, Jessup M, Bozkurt B, Butler J, Casey DE, Drazner MH, et al. 2013 ACCF/AHA guideline for the management of heart failure: a report of the American College of Cardiology Foundation/American Heart Association Task Force on Practice Guidelines. Journal of the American College of Cardiology. 2013;62(16):e147-e239.

5. Ghali JK, Krause-Steinrauf HJ, Adams KF, Khan SS, Rosenberg YD, Yancy CW, et al. Gender differences in advanced heart failure: insights from the BEST study. Journal of the American College of Cardiology. 2003;42(12):2128-34. 

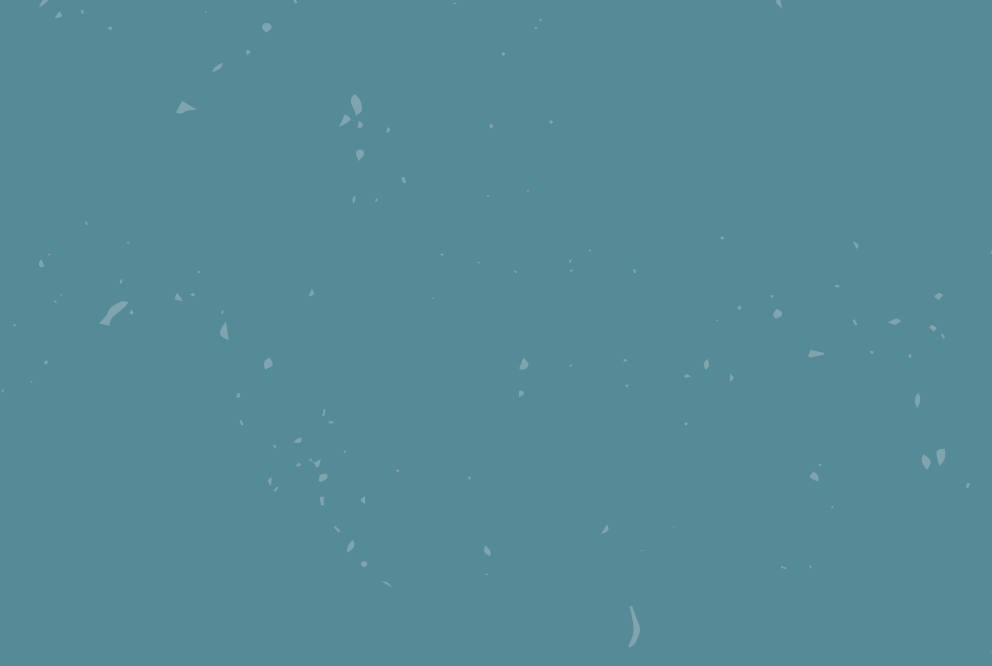

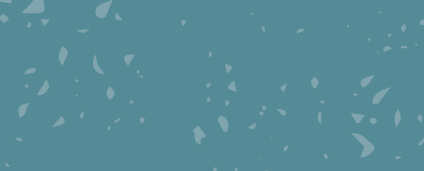




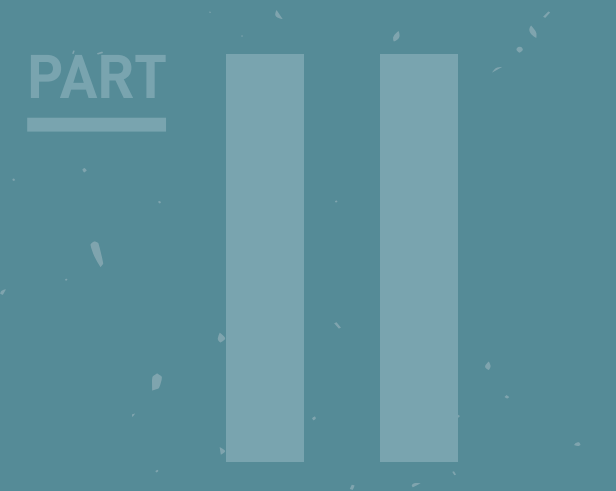

\section{Pathophysiology and Imaging}
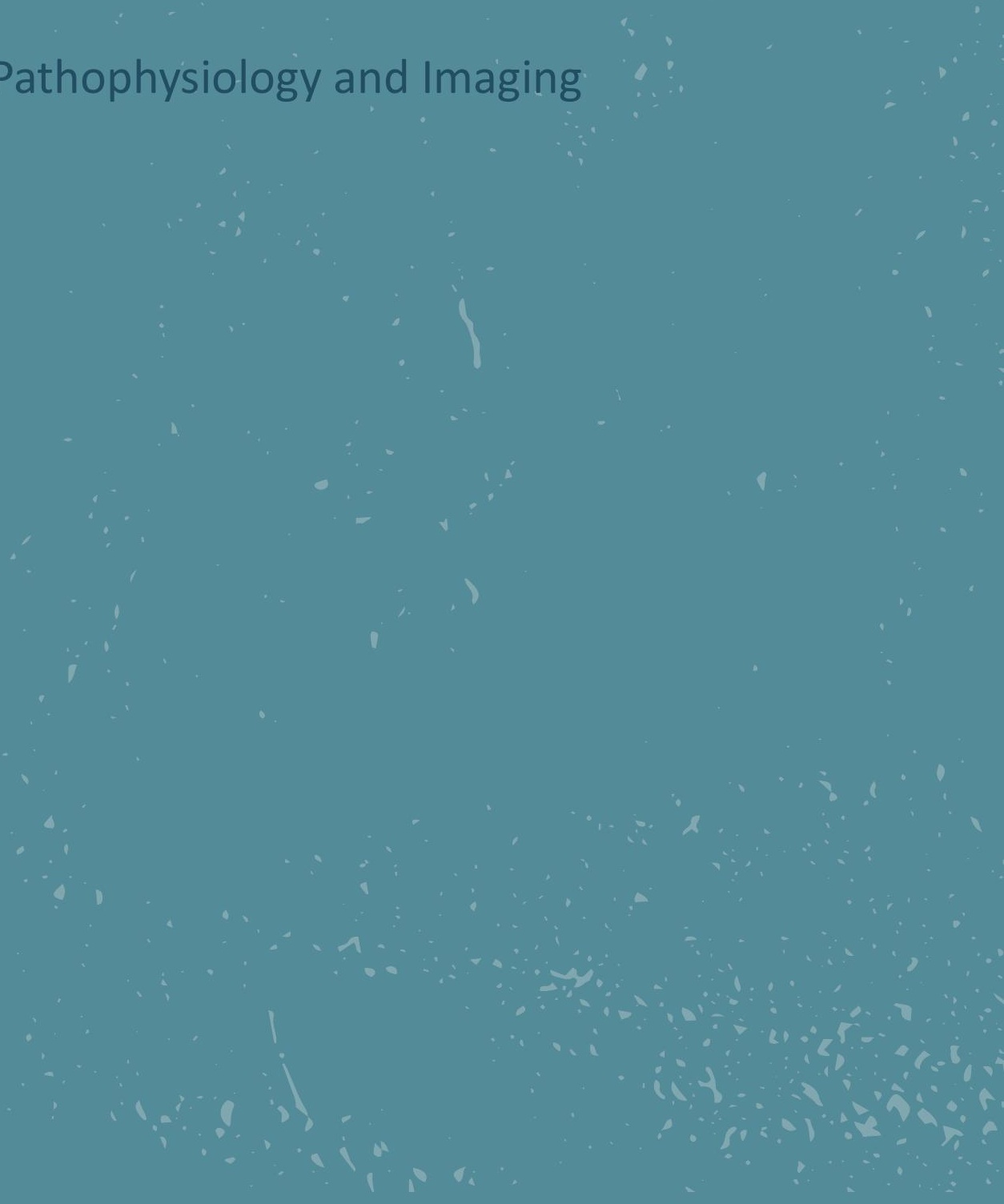


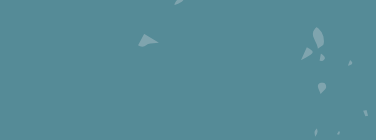

$\because \because \quad \because \therefore \quad \therefore$

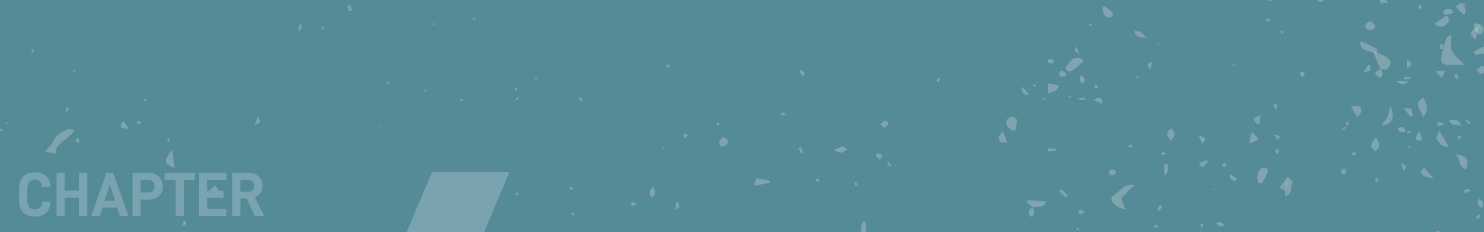
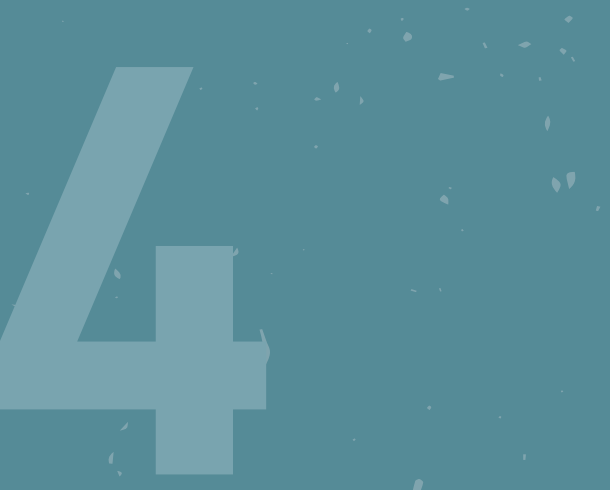

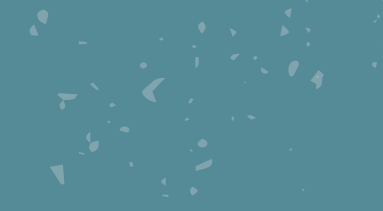

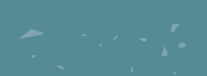

$\int_{0}^{10} \because$

$\because$

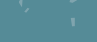

1

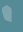

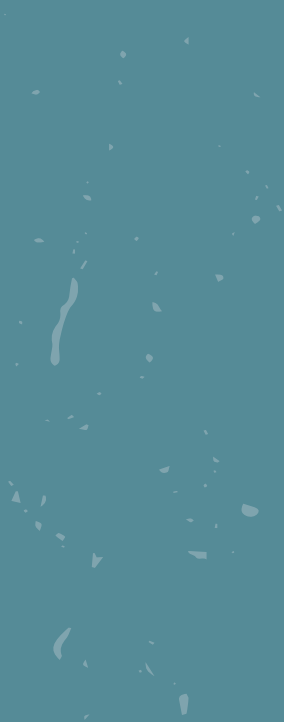

$\therefore$

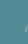

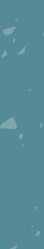

$\therefore$ 


\section{Sex-specific aspects in the pathophysiology and imaging of coronary macro- and microvascular disease}

Journal of Cardiovascular Translational Research 2020 


\section{ABSTRACT}

Sex differences in coronary artery disease (CAD) are well established, with women presenting with non-obstructive CAD more often than men do. However, recent evidence has identified coronary microvascular dysfunction as underlying cause for cardiac complaints, yet sex-specific prevalence numbers are inconclusive. This review summarizes known sex-specific aspects in the pathophysiology of both macro and microvascular dysfunction and identifies currently existing knowledge gaps. In addition, this review describes current diagnostic approaches and whether these should take underlying sex differences into account by, for example, using different techniques or cut-off values for women and men. Future research into both innovation of imaging techniques and perfusion-related sex differences is needed to fill evidence gaps and enable the implementation of the available knowledge in daily clinical practice. 


\section{INTRODUCTION}

Women suffering from coronary artery disease (CAD), one of the leading causes of death globally, have a worse short- and long-term prognosis than men $(1,2)$. They also more often present with clean epicardial arteries (non-obstructive CAD) than men (1), suggesting sex differences are present in the underlying etiology. The Women's Ischemia Syndrome Evaluation study found that women with persistent anginal complaints and non-obstructive CAD had twice the risk of cardiovascular events compared with women without complaints (3). Additionally, myocardial perfusion was impaired in approximately half of the women, suggesting that coronary microvascular dysfunction (CMD) plays an important role in the pathophysiology of this condition (4). Since endothelium-dependent dysfunction can result in decreased perfusion of the myocardium, research suggests that this may be involved in the development of CMD (5).

However, CMD remains difficult to diagnose because the cardiac microvasculature is too small to visualize with conventional imaging techniques. In addition, generalized recommendations regarding treatment for non-obstructive CAD are still lacking (6). The most recent guidelines from the European Society of Cardiology acknowledge that women with non-obstructive CAD are a special group in need of additional research (7). This review summarizes both the known sex differences in the pathophysiology of (non-)obstructive CAD and the currently available imaging tools for diagnosis, while also identifying evidence gaps and providing some future perspectives.

\section{Structural and functional alterations in macrovascular disease}

Coronary macrovascular disease or obstructive CAD occurs due to formation and/or rupture of atherosclerotic plaques. Sex differences in atherosclerosis can be observed at different levels. At the risk factor level, diabetes and smoking have a disproportionally large effect on atherosclerosis risk in women compared with men (8). Other classical risk factors such as hypertension and dyslipidemia affect the risk equally in both sexes (1).

At the structural level, animal data show that female rodents develop less extensive atherosclerosis under high-fat conditions than male rodents, possibly due to the effect of estrogens (8). Activation of estrogen receptors in female rats suppressed the proliferation of smooth muscle cells (SMCs), an effect that was not seen in males despite the presence of estrogen receptors on SMCs of both sexes $(9,10)$. This suggests that estrogens may limit the degree of structural alterations in the vasculature in a 
sex-specific manner. In addition, women more often present with plaque erosion while men more often have plaques prone to rupture (11-13). An extensive review of the (sex-specific) pathophysiology of atherosclerotic plaque formation is beyond the scope of this review and can be found elsewhere $(14,8)$.

At the functional level, atherosclerosis-related changes in the vascular wall may reduce arterial compliance, which can lead to hypertension (15). Hypertension is the most prevalent risk factor for cardiovascular diseases (16) and is more common in elderly women than men (17). Women with hypertension maintain better systolic function than men but exhibit more stiffening of both the myocardium and the vasculature, suggesting sex differences underlying mechanisms of pressure overload (17).

Clinical observations show that women are more likely to have a normal coronary angiogram (CAG) than men, both when presenting with chest pain complaints (18) or when having a confirmed diagnosis of myocardial infarction (MI) (19). Data from a sudden coronary death registry showed that $40 \%$ of the women who died from CAD did not have any thrombi while this occurred in only $28 \%$ of men (11). This apparent paradox between unobstructed epicardial arteries and poor prognosis most often seen in women may be explained by the presence of microvascular disease.

\section{Structural and functional alterations in microvascular disease}

The coronary microvasculature modulates the vascular tone through vasoconstriction and vasodilation, which is regulated by systemic and local factors acting on endothelial cells (ECs) and SMCs (20). The dysregulation of this adaptive system due to structural and functional alterations in the microvasculature is referred to as $\operatorname{CMD}(21,22)$. Classical macrovascular disease risk factors such as smoking, age and hypertension may also be associated with impaired microvascular function (23). Hypertension may disproportionally increase the CMD risk in women because they have lower microvascular arterial compliance than men (24). Sex differences in structural and functional alterations in CMD have been reported but require validation.

At the structural level, inflammation can affect the microvasculature by inducing SMC proliferation and differentiation of fibroblasts into myofibroblasts $(25,26)$. In general, nitric oxide (NO) is needed to maintain the normal functioning and structure of the arteries. NO is produced by endothelial NO synthase (eNOS) in reaction to shear stress of the artery walls, and a drop in NO levels leads to increased perivascular fibrosis and subsequent microvascular stiffening. Such a drop may occur in situations of pressure overload, when cardiomyocyte mitochondria produce free reactive oxygen species (ROS) in response to stress $(27,28)$. These free ROS induce endothelial 
inflammation, which can cause perivascular fibrosis (29). Estrogens promote the production of $\mathrm{NO}$ and may therefore protect women against structural changes in the microvasculature. However, this possible protective effect of estrogens is lost with the lack of estrogens after menopause, possibly leading to increased perivascular fibrosis and subsequent microvascular stiffening. Estrogens also inhibit collagen I and III deposition through activation of the Estrogen Receptor (ER) (30), while androgens such as testosterone promote the deposition of collagen via increasing TGF- $\beta$ production (31). This may also contribute to increased perivascular fibrosis in women after menopause.

Capillary dysfunction leads to impaired angiogenesis (32), a process regulated by several myocardium-derived growth factors stimulating endothelial cell growth (3336). ERs can act as transcription factors for one of these molecules, vascular endothelial growth factor (VEGF). Animal data show that female mice have a better angiogenic capacity following ischemia than male mice, suggesting that estrogens help limiting myocardial damage after reduced blood flow in female animals $(37,38)$, possibly via the stimulation of NO production.

Microvascular instability and dysfunction will eventually lead to pruning of vessels, called rarefaction. This decreases the myocardial capillary density, leading to reduced perfusion of the heart and possibly myocardial hypoxia. Reduced myocardial perfusion has been shown to be a major contributing factor in heart failure with preserved ejection fraction (HFpEF) in both women and men (24). The prevalence and pathophysiology of capillary rarefaction in the heart has not yet been firmly established as autopsy studies in HFpEF patients are rare. The contribution of microvascular rarefaction to CMD and possibly HFpEF in both sexes needs further investigation and might provide an interesting target for therapy in this heart failure subtype (39).

At the functional level, imbalance between vasodilating and vasoconstricting factors can lead to an impaired vessel response upon changes in oxygen demand and subsequent perfusion defects. The estrogen receptors ER- $\alpha$ and ER- $\beta$ can induce vasodilation by activating eNOS, which may have a protective effect on cardiac function. Low estrogen levels initiate sustained renin-angiotensin-aldosterone system (RAAS) activation, which promotes ROS production and further decreases NO availability (40). The lack of estrogen after menopause may thereby lead to an increase in microvascular dysregulation possibly deteriorating into CMD. This together with the presence of cardiovascular risk factors could render women more vulnerable to macro- and microvascular dysfunction after menopause. 


\section{Functional assessment of the coronary microcirculation}

While direct visualization of microvascular abnormalities is still impossible, measurements of the coronary flow enable indirect assessment of microvascular function. Under normal physiological conditions, the coronary microvasculature can induce reactive hyperemia in response to short or prolonged myocardial ischemia. In the presence of endothelium (in)dependent abnormalities, both the reactive hyperemia response and the subsequent (re)perfusion of the myocardium are suboptimal. Inducing stress can mimic this maximal hyperemic response for functional assessment of the microcirculation in the clinical setting. The difference in perfusion between healthy and diseased states can be assessed with several invasive and noninvasive imaging techniques and can inform healthcare professionals about the degree of microvascular disease.

\section{Quantification of perfusion}

Coronary blood flow can be quantified using several methods. All quantification methods require the use of either an endothelium-dependent $(41,42)$ or endotheliumindependent $(43,44)$ vasoactive stimulus to achieve maximal hyperemia. There is no evidence for sex differences in the effect of these stimuli. However, depending on how coronary perfusion is measured, inherent biological sex differences in coronary blood flow may require sex-specific cut-off values for impaired perfusion. We will discuss three commonly used myocardial perfusion metrics below. A more extensive overview can be found in Table 1.

Fractional flow reserve (FFR) is a surrogate estimate of coronary flow based on coronary pressure used to assess the extent of coronary artery stenosis. It is calculated by dividing the distal coronary artery pressure by the mean aortic pressure after maximal vasodilation. The FFR has no sex-specific cut-off point, with a value of 0.8 or higher indicating normal blood flow in both women and men. The FFR cannot differentiate between $C A D$ and $C M D$, so additional testing is required to confirm $C M D$ in case of an abnormal FFR $(45,46)$.

The index of microcirculatory resistance (IMR) is a measure of microcirculatory resistance at maximal hyperemia calculated by dividing the distal coronary pressure by the absolute coronary flow. The IMR is unaffected by resting hemodynamic parameters or epicardial stenosis and provides a more direct measurement of the coronary microcirculatory function compared to the other metrics discussed here $(47,48)$. An IMR $\geq 23-25 \mathrm{U}$ is indicative for increased microcirculatory resistance in both women and men (47). 
Table 1. Sex differences in currently available parameters to quantify coronary perfusion

\begin{tabular}{|c|c|c|c|}
\hline $\begin{array}{l}\text { Parameter } \\
\text { (abbreviation) }\end{array}$ & $\begin{array}{l}\text { Method used to calculate } \\
\text { perfusion }\end{array}$ & $\begin{array}{l}\text { Imaging modalities using } \\
\text { this parameter to quantify } \\
\text { perfusion }\end{array}$ & Sex differences \\
\hline $\begin{array}{l}\text { Fractional Flow Reserve } \\
\text { (FFR) }\end{array}$ & $\mathrm{P}_{\mathrm{d}} / \mathrm{P}_{\mathrm{a}}$ at maximal hyperemia & CAG & $\begin{array}{l}\text { None reported } \\
(47,81,82,46,49,48,83,50)\end{array}$ \\
\hline $\begin{array}{l}\text { Index of Microcirculatory } \\
\text { Resistance (IMR) }\end{array}$ & $\begin{array}{l}\mathrm{P}_{\mathrm{d}} / \text { absolute coronary flow at } \\
\text { maximal hyperemia }\end{array}$ & CAG & None reported $(46,49)$ \\
\hline $\begin{array}{l}\text { Coronary Flow Reserve } \\
\text { (CFR) }\end{array}$ & $\begin{array}{l}\text { Hyperemic coronary flow / } \\
\text { basal coronary flow }\end{array}$ & $\begin{array}{l}\text { CAG, PET, } \\
\text { echocardiography, CMR }\end{array}$ & $\begin{array}{l}\text { Ratio possibly lower in } \\
\text { women (49) }\end{array}$ \\
\hline $\begin{array}{l}\text { Myocardial Blood Flow } \\
\text { (MBF) }\end{array}$ & $\begin{array}{l}\text { Absolute myocardial perfusion } \\
\text { in } \mathrm{mL} / \mathrm{min} / \mathrm{g}\end{array}$ & PET, CMR & $\begin{array}{l}\text { Rest and stress MBF higher } \\
\text { in women. } \\
\text { MBF ratio lower in women } \\
\text { (84) }\end{array}$ \\
\hline $\begin{array}{l}\text { Myocardial Perfusion } \\
\text { Reserve Index (MPRI) }\end{array}$ & $\begin{array}{l}\text { Myocardial perfusion in stress / } \\
\text { myocardial perfusion in rest }\end{array}$ & CMR & Not yet reported \\
\hline
\end{tabular}

$\mathrm{CAG}$, coronary angiography; CMR, cardiac magnetic resonance imaging; $\mathrm{Pa}$, mean proximal coronary artery pressure (mean aortic pressure); $\mathrm{PET}$, positron emission tomography; $\mathrm{Pd}$, mean distal coronary artery pressure

Coronary flow reserve (CFR) is an estimate of coronary perfusion. It measures the maximal blood flow achieved in both epicardial and microvascular vessels in response to hyperemic stimulation and is calculated by dividing the coronary flow at maximal vasodilation by the coronary flow at rest. Women have a higher resting flow but similar hyperemic flow compared with men, leading to a lower CFR value with the same degree of microvascular dysfunction (49). As of yet there is no consensus on the cut-off for CFR to denote impaired myocardial perfusion, but the underlying sex difference in hemodynamics supports research into the use of a different value for men and women.

\section{Invasive imaging methods}

Invasive imaging measures the coronary blood flow velocity at rest and stress during coronary catheterization using either intra-coronary Doppler flow or thermodilution $(50,51)$. Impaired microvascular function measured by Doppler flow was associated with an increased risk of long-term cardiac mortality in patients with ST-elevation myocardial infarction (50) and Doppler-derived CFR correlated better with the noninvasive gold standard positron emission tomography (PET) than thermodilutionderived CFR (52). However, with thermodilution the CFR and the IMR can be obtained simultaneously (51). There are no reported differences in effectiveness of these techniques between women and men, but underlying hemodynamic differences must be taken into account when interpreting the results (49). 


\section{Non-invasive imaging methods}

There are several non-invasive imaging methods available that differ in their approach and use of radiation. PET is considered the gold standard of non-invasive imaging (53), but exposes patients to ionizing radiation. Alternatives are transthoracic Doppler echocardiography (TTDE), cardiac magnetic resonance (CMR) (54) and possibly cardiac computed tomography angiography (CCTA) $(55,56)$. These methods are summarized in Table 1. Data show that measurements obtained by TTDE correlate well with those obtained by invasive Doppler echocardiography (57-59). CCTA is currently only used for evaluation of calcification and stenosis in the epicardial vessels, but research groups are working on expanding its application to measuring myocardial perfusion and developing computational techniques that can extract flow and pressure data from CCTA images $(55,56)$. CMR can detect both perfusion defects and obstructive CAD more accurately than invasive Doppler echocardiography and single-photon emission computed tomography (SPECT), respectively $(54,60)$. It thus offers the potential to diagnose both obstructive CAD and CMD in a single examination (61). There are no reported sex differences for these imaging methods.

\section{DISCUSSION}

In this review, we summarized currently available evidence on sex differences in the pathophysiology and diagnosis of coronary macro- and microvascular disease. In contrast to obstructive CAD, knowledge about non-obstructive CAD and CMD is still lacking on many levels. At the pathophysiological level, sex differences in structural and functional alterations in CMD have been reported but remain understudied. Meanwhile at the clinical level, consensus on the preferred imaging method and perfusion quantification metric is lacking and the prevalence of $C M D$ remains unclear. In addition, perfusion defects are determined using the same cut-off values for men and women, even though research has shown that sex-specific cut-off values may be more appropriate. This unnecessary extra heterogeneity complicates the identification of true differences between women and men. Clear guideline recommendations on the choice of vasoactive stimulus, imaging medium and perfusion cut-off values will help to streamline and focus research efforts in this field.

\section{Future perspectives for CMD diagnostics}

To gain more insight into the pathophysiology and treatment of patients with nonobstructive CAD, easily accessible and low risk diagnostics are needed to identify patients with CMD. Both non-invasive imaging techniques and blood-based biomarkers may provide future diagnostics for CMD (62). High reliability and lack of radiation 
make CMR a promising non-invasive imaging technique for diagnosis of CMD. It is currently not considered as a standard diagnostic tool for CMD due to the limited availability of imaging equipment and the lack of agreement regarding acquisition and post-processing. Research efforts aimed at facilitating the use of CMR in standard care are working on creating perfusion measurements without the use of contrast injection (63), creating a fully automated absolute perfusion measurement (64), and building new MRI coils that will reduce MRI scanning times from an hour to 15 minutes (65). These improvements will make CMR a more feasible and attractive diagnostic option for CMD in the future. Machine learning algorithms that support the imaging specialist in interpreting imaging results can be implemented to improve the accuracy of the diagnosis while reducing the reading time (66). Machine learning can also be applied to clinical care data. Algorithms built using data from electronic health records are emerging as a tool to help clinicians translate the substantial amount of available data to a diagnosis and appropriate treatment (67). It is important to stress the use of a sex-specific approach in validating these algorithms, since they are only as unbiased as the data they are based upon. If women are underrepresented in the datasets used to power these models, the algorithm could perform poorly for women. For example, facial recognition software based on an unbalanced dataset used classifiers that performed better on male faces than female faces (68). Therefore, proportionate representation of both women and men, but also of ethnic groups, should be ensured before using a dataset to develop healthcare algorithms (69).

Biomarkers can be used to both improve risk stratification for and diagnosis of patients with CAD, possibly reducing the need for imaging in these patients. Several different markers of vascular inflammation, oxidative stress and some others have been proposed as possible biomarkers for CMD, but have not been established or validated yet (62).

\section{Treatment perspectives and related diseases}

Treatment for obstructive CAD is well established and includes revascularization via stenting or coronary bypass grafting. Data show that women are less likely to undergo these interventions than men, even when they have been diagnosed with acute coronary syndrome (ACS) (19) and have an equal or even higher risk profile (70). This sex difference is also apparent in prescribed medication, as women with ACS were less likely to receive $\beta$-blockers and statins than men with similar disease severity (71). Women taking cardiovascular medications are more likely to experience (serious) adverse drug reactions than men (72), which may explain why physicians may choose not to prescribe these drugs for women. However, sex-specific evidence per separate drug class is still too limited (73) to support not prescribing these drugs for women and thereby denying them the advantages of treatment. 
Treatment for CMD has been largely empirical due to the lack of knowledge about the pathophysiology and the difficulty of reliably diagnosing the condition. Currently available options include medications already used to treat obstructive CAD and cardiovascular risk factors, such as low-dose aspirin, statins and $\beta$-blockers (74). Persistent anginal symptoms can be reduced by using a device to narrow the coronary sinus (75). Optimal treatment of CMD is important, as several studies have shown that impaired perfusion of the heart is related to a poor prognosis independent of the imaging modality used (76-78). Given that the currently prescribed medications have already been in use for other indications, it is likely that the sex differences described for obstructive CAD treatment also hold true for CMD treatment. However, data on this are still lacking due to the novelty of the research field.

CMD can be the precursor of chronic cardiac conditions such as HFpEF (54), a subtype of HF that is more common in women (79). The prognosis of HFpEF is poor with approximately half of patients dying within five years after diagnosis but effective treatments are still lacking (79). Better understanding and earlier recognition of subclinical conditions such as CMD are therefore crucial to tackle this syndrome early on. Next to improvement of diagnosis of CMD, more research is needed into possible treatments of CMD, the underlying pathophysiology and possible disease phenotypes that can identify subgroups at higher or lower risk of developing HFpEF (80).

\section{CONCLUSION}

Sex differences in CAD have been identified at all levels of the disease, but such detailed information is still missing for CMD. While research has suggested the presences of such differences, for example through the effect of sex hormones, many evidence gaps still exist. Currently available imaging techniques enable clinicians to evaluate CMD, but international consensus on the optimal procedure is missing and underlying sex differences in baseline perfusion are not always taken into account. Innovative strategies to improve current diagnostic techniques such as the incorporation of machine learning approaches will hopefully enable clinicians to screen for CMD in standard care. However, these approaches must consider sex differences in their development to avoid the introduction of biases in the end product. 


\section{Sources of Funding}

This study was funded by the Dutch Heart Foundation (2013T084, Queen of Hearts Program) and by ZonMw grant (849100003, Reviews en Kennissyntheses Gender en Gezondheid). 


\section{REFERENCES}

1. Parvand, M., Rayner-Hartley, E., \& Sedlak, T. (2018). Recent Developments in Sex-Related Differences in Presentation, Prognosis, and Management of Coronary Artery Disease. Canadian Journal of Cardiology, 34(4), 390-399, doi:https://doi.org/10.1016/j.cjca.2018.01.007.

2. Izadnegahdar, M., Mackay, M., Lee, M. K., Sedlak, T. L., Gao, M., Bairey Merz, C. N., et al. (2016). Sex and ethnic differences in outcomes of acute coronary syndrome and stable angina patients with obstructive coronary artery disease. Circulation: Cardiovascular Quality and Outcomes, 9(2_suppl_1), S26-S35.

3. Gulati, M., Cooper-DeHoff, R. M., McClure, C., Johnson, B. D., Shaw, L. J., Handberg, E. M., et al. (2009). Adverse cardiovascular outcomes in women with nonobstructive coronary artery disease: a report from the Women's Ischemia Syndrome Evaluation Study and the St James Women Take Heart Project. Archives of internal medicine, 169(9), 843-850, doi:10.1001/archinternmed.2009.50.

4. Reis, S. E., Holubkov, R., Smith, A. C., Kelsey, S. F., Sharaf, B. L., Reichek, N., et al. (2001). Coronary microvascular dysfunction is highly prevalent in women with chest pain in the absence of coronary artery disease: results from the NHLBI WISE study. American heart journal, 141(5), 735-741.

5. Bairey Merz, C. N., Pepine, C. J., Walsh, M. N., Fleg, J. L., Camici, P. G., Chilian, W. M., et al. (2017). Ischemia and No Obstructive Coronary Artery Disease (INOCA) Developing Evidence-Based Therapies and Research Agenda for the Next Decade. Circulation, 135(11), 1075-1092.

6. Paul, T. K., Sivanesan, K., \& Schulman-Marcus, J. (2017). Sex differences in nonobstructive coronary artery disease: Recent insights and substantial knowledge gaps. Trends in Cardiovascular Medicine, 27(3), 173-179, doi:https:// doi.org/10.1016/j.tcm.2016.08.002.

7. Montalescot, G., Sechtem, U., Achenbach, S., Andreotti, F., Arden, C., Budaj, A., et al. (2013). 2013 ESC guidelines on the management of stable coronary artery diseaseThe Task Force on the management of stable coronary artery disease of the European Society of Cardiology. European Heart Journal, 34(38), 2949-3003, doi:10.1093/eurheartj/ eht296.

8. Mathur, P., Ostadal, B., Romeo, F., \& Mehta, J. L. (2015). Gender-Related Differences in Atherosclerosis. [journal article]. Cardiovascular Drugs and Therapy, 29(4), 319-327, doi:10.1007/s10557-015-6596-3.

9. Szego, C. M., \& Davis, J. S. (1967). Adenosine 3', 5'-monophosphate in rat uterus: acute elevation by estrogen. Proceedings of the National Academy of Sciences, 58(4), 1711-1718.

10. Hogg, M. E., Vavra, A. K., Banerjee, M. N., Martinez, J., Jiang, Q., Keefer, L. K., et al. (2012). The Role of Estrogen Receptor $\alpha$ and $\beta$ in Regulating Vascular Smooth Muscle Cell Proliferation is Based on Sex1. Journal of Surgical Research, 173(1), e1-e10.

11. Yahagi, K., Davis, H. R., Arbustini, E., \& Virmani, R. (2015). Sex differences in coronary artery disease: Pathological observations. Atherosclerosis, 239(1), 260-267, doi:https://doi.org/10.1016/j.atherosclerosis.2015.01.017.

12. Kataoka, Y., Puri, R., Hammadah, M., Duggal, B., Uno, K., Kapadia, S. R., et al. (2016). Sex Differences in Nonculprit Coronary Plaque Microstructures on Frequency-Domain Optical Coherence Tomography in Acute Coronary Syndromes and Stable Coronary Artery Disease. Circulation: Cardiovascular Imaging, 9(8), e004506, doi:doi:10.1161/CIRCIMAGING.116.004506.

13. Hellings, W. E., Pasterkamp, G., Verhoeven, B. A. N., De Kleijn, D. P. V., De Vries, J.-P. P. M., Seldenrijk, K. A., et al. (2007). Gender-associated differences in plaque phenotype of patients undergoing carotid endarterectomy. Journal of vascular surgery, 45(2), 289-296, doi:10.1016/j.jvs.2006.09.051.

14. Libby, P., Ridker, P. M., \& Hansson, G. K. (2011). Progress and challenges in translating the biology of atherosclerosis. Nature, 473,317 , doi:10.1038/nature10146.

15. Hansen, L., \& Taylor, W. R. (2016). Is increased arterial stiffness a cause or consequence of atherosclerosis? Atherosclerosis, 249, 226-227.

16. Fisher, N. D. L., \& Curfman, G. (2018). Hypertension-A Public Health Challenge of Global ProportionsHypertension-A Public Health Challenge of Global ProportionsEditorial. Jama, 320(17), 1757-1759, doi:10.1001/ jama.2018.16760.

17. Regitz-Zagrosek, V., \& Kararigas, G. (2017). Mechanistic Pathways of Sex Differences in Cardiovascular Disease. Physiological reviews, 97(1), 1-37, doi:10.1152/physrev.00021.2015.

18. Sullivan, A. K., Holdright, D. R., Wright, C. A., Sparrow, J. L., Cunningham, D., \& Fox, K. M. (1994). Chest pain in women: clinical, investigative, and prognostic features. Bmj, 308(6933), 883-886.

19. Hvelplund, A., Galatius, S., Madsen, M., Rasmussen, J. N., Rasmussen, S., Madsen, J. K., et al. (2010). Women with acute coronary syndrome are less invasively examined and subsequently less treated than men. European Heart 
Journal, 31(6), 684-690, doi:10.1093/eurheartj/ehp493.

20. Shaw, J., \& Anderson, T. (2016). Coronary endothelial dysfunction in non-obstructive coronary artery disease: Risk, pathogenesis, diagnosis and therapy. Vascular Medicine, 21(2), 146-155.

21. Camici, P. G., D’Amati, G., \& Rimoldi, O. (2015). Coronary microvascular dysfunction: Mechanisms and functional assessment. [Review]. Nature Reviews Cardiology, 12(1), 48-62, doi:10.1038/nrcardio.2014.160.

22. Murthy, V. L., Naya, M., Taqueti, V. R., Foster, C. R., Gaber, M., Hainer, J., et al. (2014). Effects of sex on coronary microvascular dysfunction and cardiac outcomes. [Article]. Circulation, 129(24), 2518-2527, doi:10.1161/CIRCULATIONAHA.113.008507.

23. Mygind, N. D., Michelsen, M. M., Pena, A., Frestad, D., Dose, N., Aziz, A., et al. (2016). Coronary microvascular function and cardiovascular risk factors in women with angina pectoris and no obstructive coronary artery disease: the iPOWER study. J Am Heart Assoc, 5(3), e003064.

24. Coutinho, T., Mielniczuk, L. M., Srivaratharajah, K., Wells, G. A., \& Beanlands, R. S. (2018). Coronary artery microvascular dysfunction: Role of sex and arterial load. International Journal of Cardiology, 270, 42-47.

25. Baum, J., \& Duffy, H. S. (2011). Fibroblasts and myofibroblasts: what are we talking about? Journal of cardiovascular pharmacology, 57(4), 376.

26. Suwanabol, P. A., Seedial, S. M., Shi, X., Zhang, F., Yamanouchi, D., Roenneburg, D., et al. (2012). Transforming growth factor- $\beta$ increases vascular smooth muscle cell proliferation through the Smad3 and extracellular signalregulated kinase mitogen-activated protein kinases pathways. Journal of vascular surgery, 56(2), 446-454. e441.

27. Sansone, R., Stanske, B., Keymel, S., Schuler, D., Horn, P., Saeed, D., et al. (2015). Macrovascular and microvascular function after implantation of left ventricular assist devices in end-stage heart failure: role of microparticles. The Journal of Heart and Lung Transplantation, 34(7), 921-932.

28. Schwarzer, M., Osterholt, M., Lunkenbein, A., Schrepper, A., Amorim, P., \& Doenst, T. (2014). Mitochondrial reactive oxygen species production and respiratory complex activity in rats with pressure overload-induced heart failure. The Journal of physiology, 592(17), 3767-3782.

29. Dai, Z., Aoki, T., Fukumoto, Y., \& Shimokawa, H. (2012). Coronary perivascular fibrosis is associated with impairment of coronary blood flow in patients with non-ischemic heart failure. Journal of Cardiology, 60(5), 416-421.

30. Dworatzek, E., Mahmoodzadeh, S., Schriever, C., Kusumoto, K., Kramer, L., Santos, G., et al. (2018). Sex-specific regulation of collagen I and III expression by $17 \beta$-Estradiol in cardiac fibroblasts: role of estrogen receptors. Cardiovascular Research, 115(2), 315-327.

31. Kong, P., Christia, P., \& Frangogiannis, N. G. (2014). The pathogenesis of cardiac fibrosis. Cellular and molecular life sciences, 71(4), 549-574.

32. Oka, T., Akazawa, H., Naito, A. T., \& Komuro, I. (2014). Angiogenesis and cardiac hypertrophy: maintenance of cardiac function and causative roles in heart failure. Circulation research, 114(3), 565-571.

33. Andrae, J., Gallini, R., \& Betsholtz, C. (2008). Role of platelet-derived growth factors in physiology and medicine. Genes \& development, 22(10), 1276-1312.

34. Chen, J.-X., Zeng, H., Reese, J., Aschner, J. L., \& Meyrick, B. (2012). Overexpression of angiopoietin-2 impairs myocardial angiogenesis and exacerbates cardiac fibrosis in the diabetic $\mathrm{db} / \mathrm{db}$ mouse model. American Journal of Physiology-Heart and Circulatory Physiology, 302(4), H1003.

35. Dobaczewski, M., Chen, W., \& Frangogiannis, N. G. (2011). Transforming growth factor (TGF)- $\beta$ signaling in cardiac remodeling. Journal of molecular and cellular cardiology, 51(4), 600-606.

36. Jeansson, M., Gawlik, A., Anderson, G., Li, C., Kerjaschki, D., Henkelman, M., et al. (2011). Angiopoietin-1 is essential in mouse vasculature during development and in response to injury. The Journal of clinical investigation, 121(6), 2278-2289.

37. Mahmoodzadeh, S., Leber, J., Zhang, X., Jaisser, F., Messaoudi, S., Morano, I., et al. (2014). Cardiomyocyte-specific estrogen receptor alpha increases angiogenesis, lymphangiogenesis and reduces fibrosis in the female mouse heart post-myocardial infarction. Journal of cell science \& therapy, 5(1), 153.

38. Buteau-Lozano, H., Ancelin, M., Lardeux, B., Milanini, J., \& Perrot-Applanat, M. (2002). Transcriptional regulation of vascular endothelial growth factor by estradiol and tamoxifen in breast cancer cells: a complex interplay between estrogen receptors $\alpha$ and $\beta$. Cancer Research, 62(17), 4977-4984.

39. Mohammed, S. F., Hussain, S., Mirzoyev, S. A., Edwards, W. D., Maleszewski, J. J., \& Redfield, M. M. (2015). Coronary microvascular rarefaction and myocardial fibrosis in heart failure with preserved ejection fraction. Circulation, 131(6), 550-559.

40. Beale, A. L., Meyer, P., Marwick, T. H., Lam, C. S., \& Kaye, D. M. (2018). Sex differences in cardiovascular pathophysiology: Why women are overrepresented in heart failure with preserved ejection fraction. Circulation, 138(2), 198-205. 
41. Hwang, H. J., Chung, W. B., Park, J. H., Oh, S. S., Chung, J. W., Choi, Y. S., et al. (2010). Estimation of coronary flow velocity reserve using transthoracic Doppler echocardiography and cold pressor test might be useful for detecting of patients with variant angina. Echocardiography, 27(4), 435-441.

42. Quyyumi, A. A., Dakak, N., Mulcahy, D., Andrews, N. P., Husain, S., Panza, J. A., et al. (1997). Nitric oxide activity in the atherosclerotic human coronary circulation. Journal of the American College of Cardiology, 29(2), 308-317.

43. Al Jaroudi, W., \& Iskandrian, A. E. (2009). Regadenoson: a new myocardial stress agent. Journal of the American College of Cardiology, 54(13), 1123-1130.

44. Youn, H.-J., \& Foster, E. (2004). Demonstration of coronary artery flow using transthoracic Doppler echocardiography. Journal of the American Society of Echocardiography, 17(2), 178-185.

45. De Bruyne, B., Pijls, N. H., Barbato, E., Bartunek, J., Bech, J.-W., Wijns, W., et al. (2003). Intracoronary and intravenous adenosine 5 ? -triphosphate, adenosine, papaverine, and contrast medium to assess fractional flow reserve in humans. Circulation, 107(14), 1877-1883.

46. Kern, M. J., Lerman, A., Bech, J.-W., Bruyne, B. D., Eeckhout, E., Fearon, W. F., et al. (2006). Physiological Assessment of Coronary Artery Disease in the Cardiac Catheterization Laboratory. Circulation, 114(12), 1321-1341, doi:doi:10.1161/CIRCULATIONAHA.106.177276.

47. Fearon, W. F., Balsam, L. B., Farouque, H. O., Robbins, R. C., Fitzgerald, P. J., Yock, P. G., et al. (2003). Novel index for invasively assessing the coronary microcirculation. Circulation, 107(25), 3129-3132.

48. Ng, M. K., Yeung, A. C., \& Fearon, W. F. (2006). Invasive assessment of the coronary microcirculation: superior reproducibility and less hemodynamic dependence of index of microcirculatory resistance compared with coronary flow reserve. Circulation, 113(17), 2054-2061.

49. Kobayashi, Y., Fearon, W. F., Honda, Y., Tanaka, S., Pargaonkar, V., Fitzgerald, P. J., et al. (2015). Effect of sex differences on invasive measures of coronary microvascular dysfunction in patients with angina in the absence of obstructive coronary artery disease. JACC: Cardiovascular Interventions, 8(11), 1433-1441.

50. van de Hoef, T. P., Bax, M., Meuwissen, M., Damman, P., Delewi, R., de Winter, R. J., et al. (2013). Impact of Coronary Microvascular Function on Long-term Cardiac Mortality in Patients With Acute ST-Segment-Elevation Myocardial Infarction. Circulation: Cardiovascular Interventions, 6(3), 207-215.

51. Pijls, N. H. J., Bruyne, B. D., Smith, L., Aarnoudse, W., Barbato, E., Bartunek, J., et al. (2002). Coronary Thermodilution to Assess Flow Reserve. Circulation, 105(21), 2482-2486, doi:doi:10.1161/01.CIR.0000017199.09457.3D.

52. Everaars, H., de Waard, G. A., Driessen, R. S., Danad, I., van de Ven, P. M., Raijmakers, P. G., et al. (2018). Doppler flow velocity and thermodilution to assess coronary flow reserve: a head-to-head comparison with [15O] H2O PET. JACC: Cardiovascular Interventions, 11(20), 2044-2054.

53. Driessen, R. S., Raijmakers, P. G., Stuijfzand, W. J., \& Knaapen, P. (2017). Myocardial perfusion imaging with PET. The international journal of cardiovascular imaging, 33(7), 1021-1031.

54. Paulus, W. J., \& Tschope, C. (2013). A novel paradigm for heart failure with preserved ejection fraction: comorbidities drive myocardial dysfunction and remodeling through coronary microvascular endothelial inflammation. J Am Coll Cardiol, 62(4), 263-271, doi:10.1016/j.jacc.2013.02.092.

55. Celeng, C., Leiner, T., Maurovich-Horvat, P., Merkely, B., de Jong, P., Dankbaar, J. W., et al. (2018). Anatomical and Functional Computed Tomography for Diagnosing Hemodynamically Significant Coronary Artery Disease: A Meta-Analysis. JACC Cardiovasc Imaging, doi:10.1016/j.jcmg.2018.07.022.

56. Nakazato, R., Heo, R., Leipsic, J., \& Min, J. K. (2014). CFR and FFR assessment with PET and CTA: strengths and limitations. Current cardiology reports, 16(5), 484.

57. Caiati, C., Montaldo, C., Zedda, N., Montisci, R., Ruscazio, M., Lai, G., et al. (1999). Validation of a new noninvasive method (contrast-enhanced transthoracic second harmonic echo Doppler) for the evaluation of coronary flow reserve: comparison with intracoronary Doppler flow wire. Journal of the American College of Cardiology, 34(4), $1193-1200$.

58. Caiati, C., Zedda, N., Montaldo, C., Montisci, R., \& Iliceto, S. (1999). Contrast-enhanced transthoracic second harmonic echo Doppler with adenosine: a noninvasive, rapid and effective method for coronary flow reserve assessment. Journal of the American College of Cardiology, 34(1), 122-130.

59. Hozumi, T., Yoshida, K., Akasaka, T., Asami, Y., Ogata, Y., Takagi, T., et al. (1998). Noninvasive assessment of coronary flow velocity and coronary flow velocity reserve in the left anterior descending coronary artery by Doppler echocardiography: comparison with invasive technique. Journal of the American College of Cardiology, 32(5), 1251-1259.

60. Greenwood, J. P., Maredia, N., Younger, J. F., Brown, J. M., Nixon, J., Everett, C. C., et al. (2012). Cardiovascular magnetic resonance and single-photon emission computed tomography for diagnosis of coronary heart disease (CE-MARC): a prospective trial. The Lancet, 379(9814), 453-460. 
61. Liu, A., Wijesurendra, R. S., Liu, J. M., Forfar, J. C., Channon, K. M., Jerosch-Herold, M., et al. (2018). Diagnosis of microvascular angina using cardiac magnetic resonance. Journal of the American College of Cardiology, 71(9), 969-979.

62. Hung, O. Y., Lee, S. K., Eshtehardi, P., \& Samady, H. (2016). Novel biomarkers of coronary microvascular disease. Future Cardiology, 12(4), 497-509.

63. Liu, A., Wijesurendra, R. S., Francis, J. M., Robson, M. D., Neubauer, S., Piechnik, S. K., et al. (2016). Adenosine stress and rest T1 mapping can differentiate between ischemic, infarcted, remote, and normal myocardium without the need for gadolinium contrast agents. JACC: Cardiovascular Imaging, 9(1), 27-36.

64. Jerosch-Herold, M., Swingen, C., \& Seethamraju, R. T. (2002). Myocardial blood flow quantification with MRI by model-independent deconvolution. Medical physics, 29(5), 886-897.

65. Gruber, B., Hendriks, A. D., Alborahal, C., Strijkers, G. J., Klomp, D. W. J., Leiner, T., et al. (2018). A 256-channel Cardiac Coil for accelerated Cardiac Imaging at 3 Tesla - Evaluation of a 32-channel Prototype. 26, 4290.

66. Wang, S., \& Summers, R. M. (2012). Machine learning and radiology. Medical Image Analysis, 16(5), 933-951, doi:https://doi.org/10.1016/j.media.2012.02.005.

67. Moja, L., Kwag, K. H., Lytras, T., Bertizzolo, L., Brandt, L., Pecoraro, V., et al. (2014). Effectiveness of computerized decision support systems linked to electronic health records: a systematic review and meta-analysis. American journal of public health, 104(12), e12-e22.

68. Buolamwini, J., \& Gebru, T. Gender shades: Intersectional accuracy disparities in commercial gender classification. In Conference on Fairness, Accountability and Transparency, 2018 (pp. 77-91)

69. Leavy, S. Gender bias in artificial intelligence: The need for diversity and gender theory in machine learning. In Proceedings of the 1st International Workshop on Gender Equality in Software Engineering, 2018 (pp. 14-16): ACM

70. Worrall-Carter, L., McEvedy, S., Kuhn, L., Scruth, E., Maclsaac, A., \& Rahman, M. A. (2017). Systematic Review and Meta-analyses Investigating Whether Risk Stratification Explains Lower Rates of Coronary Angiography Among Women With Non-ST-Segment Elevation Acute Coronary Syndrome. J Cardiovasc Nurs, 32(2), 112-124, doi:10.1097/jen.0000000000000300.

71. Dey, S., Flather, M. D., Devlin, G., Brieger, D., Gurfinkel, E. P., Steg, P. G., et al. (2009). Sex-related differences in the presentation, treatment and outcomes among patients with acute coronary syndromes: the Global Registry of Acute Coronary Events. Heart, 95(1), 20-26, doi:10.1136/hrt.2007.138537.

72. Rosano, G. M., Lewis, B., Agewall, S., Wassmann, S., Vitale, C., Schmidt, H., et al. (2015). Gender differences in the effect of cardiovascular drugs: a position document of the Working Group on Pharmacology and Drug Therapy of the ESC. Eur Heart J, 36(40), 2677-2680, doi:10.1093/eurheartj/ehv161.

73. Bots, S. H., Groepenhoff, F., Eikendal, A. L. M., Tannenbaum, C., Rochon, P. A., Regitz-Zagrosek, V., et al. (2019). Adverse Drug Reactions to Guideline-Recommended Heart Failure Drugs in Women. A Systematic Review of the Literature, 7(3), 258-266, doi:10.1016/j.jchf.2019.01.009.

74. Taqueti, V. R., \& Di Carli, M. F. (2018). Coronary Microvascular Disease Pathogenic Mechanisms and Therapeutic Options., 72(21), 2625-2641, doi:10.1016/j.jacc.2018.09.042.

75. Verheye, S., Jolicoeur, E. M., Behan, M. W., Pettersson, T., Sainsbury, P., Hill, J., et al. (2015). Efficacy of a device to narrow the coronary sinus in refractory angina. N Engl J Med, 372(6), 519-527, doi:10.1056/NEJMoa1402556.

76. Brainin, P., Frestad, D., \& Prescott, E. (2018). The prognostic value of coronary endothelial and microvascular dysfunction in subjects with normal or non-obstructive coronary artery disease: A systematic review and metaanalysis. [Article]. International Journal of Cardiology, 254, 1-9, doi:10.1016/j.ijcard.2017.10.052.

77. Indorkar, R., Kwong, R. Y., Romano, S., White, B. E., Chia, R. C., Trybula, M., et al. (2018). Global Coronary Flow Reserve Measured During Stress Cardiac Magnetic Resonance Imaging Is an Independent Predictor of Adverse Cardiovascular Events. [Article in Press]. JACC: Cardiovascular Imaging, doi:10.1016/j.jcmg.2018.08.018.

78. Lee, J. M., Jung, J. H., Hwang, D., Park, J., Fan, Y., Na, S. H., et al. (2016). Coronary Flow Reserve and Microcirculatory Resistance in Patients with Intermediate Coronary Stenosis. [Article]. Journal of the American College of Cardiology, 67(10), 1158-1169, doi:10.1016/j.jacc.2015.12.053.

79. Dunlay, S. M., Roger, V. L., \& Redfield, M. M. (2017). Epidemiology of heart failure with preserved ejection fraction. Nat Rev Cardiol, 14(10), 591-602, doi:10.1038/nrcardio.2017.65.

80. Shah, S. J., Katz, D. H., Selvaraj, S., Burke, M. A., Yancy, C. W., Gheorghiade, M., et al. (2015). Phenomapping for novel classification of heart failure with preserved ejection fraction. Circulation, 131(3), 269-279, doi:10.1161/ circulationaha.114.010637.

81. Johnson, N. P., \& Gould, K. L. (2011). Physiological basis for angina and ST-segment change: PET-verified thresholds of quantitative stress myocardial perfusion and coronary flow reserve. JACC: Cardiovascular Imaging, 4(9), 990-998. 
82. Kern, M. J. (2000). Coronary physiology revisited: practical insights from the cardiac catheterization laboratory. Circulation, 101(11), 1344-1351.

83. Sara, J. D., Widmer, R. J., Matsuzawa, Y., Lennon, R. J., Lerman, L. O., \& Lerman, A. (2015). Prevalence of Coronary Microvascular Dysfunction Among Patients With Chest Pain and Nonobstructive Coronary Artery Disease. JACC: Cardiovascular Interventions, 8(11), 1445-1453, doi:https://doi.org/10.1016/j.jcin.2015.06.017.

84. Opstal, T., Knol, R., Cornel, J., Wondergem, M., \& van der Zant, F. (2018). Myocardial blood flow and myocardial flow reserve values in $13 \mathrm{~N}$-ammonia myocardial perfusion $\mathrm{PET} / \mathrm{CT}$ using a time-efficient protocol in patients without coronary artery disease. European Journal of Hybrid Imaging, 2(1), 11. 


$$
-5
$$




\section{Cardiovascular imaging of women and men visiting the outpatient clinic with chest pain or discomfort: design and rationale of the ARGUS study}

BMJ Open 2020 


\section{ABSTRACT}

Chest pain or discomfort affects 20 - 40\% of the general population over the course of their life and may be a symptom of myocardial ischemia. For the diagnosis of obstructive macrovascular coronary artery disease (CAD) algorithms have been developed, however, these do not exclude microvascular angina (MVA). This may lead to false reassurance of symptomatic patients, mainly women, with functionally significant, yet non-obstructive coronary vascular disease. Therefore, this study aims to estimate the prevalence of both macro- and microvascular coronary vascular disease in women and men presenting with chest pain or discomfort, and to subsequently develop a decision support tool to aid cardiologists in referral to cardiovascular imaging for both macro- and microvascular CAD evaluation. Women and men with chest pain or discomfort, aged 45 years and older, without a history of cardiovascular disease, who are referred to an outpatient cardiology clinic by their general practitioner are eligible for inclusion. Coronary computed tomography angiography (CCTA) is used for anatomical imaging. Additionally, myocardial perfusion imaging by adenosine stress cardiac magnetic resonance (CMR) imaging is performed to detect functionally significant coronary vascular disease. Electronic health record data, collected during regular cardiac workup, including medical history, cardiovascular risk factors, physical examination, echocardiography, (exercise) electrocardiography and blood samples for standard cardiovascular biomarkers and research purposes are obtained. Participants will be classified as positive or negative for coronary vascular disease based on all available data by expert panel consensus (a cardiovascular radiologist and two cardiologists). After completion of the clinical study, all collected data will be used to develop a decision support tool using predictive modeling and machine learning techniques. The study protocol was approved by the Institutional Review Board of the University Medical Center Utrecht. Results will be disseminated through national and international conferences and in peer-reviewed journals in cardiovascular disease.

\section{Trial registration}

trialregister.nl NL8702 


\section{INTRODUCTION}

Chest pain or discomfort affects 20 - 40\% of the general population over the span of their life and may be a symptom of myocardial ischemia (1). Approximately $40 \%$ of these patients are referred to an outpatient cardiology clinic for cardiac workup to evaluate if the underlying cause is indeed myocardial ischemia (1). However, distinguishing a possibly life-threatening cardiac etiology from a non-cardiac cause in a short period of time remains a challenge.

Current clinical diagnostic decision support tools have been solely developed to aid the cardiologist in diagnosing or excluding myocardial ischemia due to obstructive, i.e. anatomical, coronary artery disease (CAD) (2-5). Coronary computed tomography angiography (CCTA) is well-established to identify (non-)obstructive burden of anatomical coronary atherosclerotic disease and provides important information regarding CAD risk, also in women (6). However, invasive imaging studies in symptomatic patients reveal a high prevalence (63-71\%) of anatomically nonobstructive, yet functionally significant, CAD (7-9). Non-obstructive CAD has been considered a benign condition for years, but recently appeared to be a significant cause of myocardial ischemia due to observations of coronary vasospasms and microvascular angina (MVA) $(10,11)$. Furthermore, the prognosis for both sexes regarding non-obstructive CAD appears to be as poor as that of patients with obstructive $\operatorname{CAD}(12,13)$.

Despite increased awareness of the importance of coronary function testing for MVA and coronary spasms, its assessment remains a challenge carrying non-negligible risks and costs. However, recently several non-invasive imaging techniques have provided surrogate measures of the coronary microcirculation (14). Yet, the used imaging modality highly depends on local expertise, availability and costs (15). Cardiac magnetic resonance (CMR) imaging is emerging as a non-invasive technique with high spatial resolution to assess myocardial perfusion, i.e. functional CAD, without the need for ionizing radiation $(16,17)$. In fact, Knuuti et al. confirmed CMR to perform well for both anatomical and functional CAD (18). Therefore, 2019 ESC recommendations mention stress CMR imaging as an option that may be considered in patients with suspected coronary vascular disease (9).

Hence, to add to the existing knowledge regarding obstructive CAD in symptomatic patients clinically referred for imaging, this study will assess the prevalence of both anatomical obstructive (using CCTA) and (non-)obstructive, yet significant, functional CAD (using stress CMR imaging) in all patients with chest pain or discomfort visiting 
the outpatient cardiology clinics. Additionally, we aim to develop a decision support tool that aids cardiologists in referral for either CCTA and/or stress CMR imaging and study whether extensive phenotyping could improve long-term outcomes.

\section{METHODS AND ANALYSIS}

\section{Study design}

ARGUS is a single center, prospective cohort study performed at the University Medical Center Utrecht in the Netherlands. The study is actively recruiting women and men who visit cardiac outpatient clinics in Utrecht with chest pain or discomfort as the main reason for their visit. Currently, the Cardiology Centers of the Netherlands (CCN), location Utrecht, and HeartLife Klinieken Utrecht are referring centers for participant recruitment. The study procedures are conducted at the Radiology department of the UMC Utrecht.

\section{Study population}

The study population comprises women and men aged 45 years and older with chest pain or discomfort as primary complaint who are referred by their general practitioner to the participating outpatient cardiology clinics. Exclusion criteria are listed in Table 1 and include cardiac history and contraindications to undergo any of the study procedures. If CCTA or stress CMR is contraindicated after part of the study protocol is already completed, the participant will not be excluded and collected data will be used in the study.

Table 1. Inclusion and exclusion criteria for the ARGUS study

\begin{tabular}{l} 
Inclusion criteria \\
\hline 1. Age $\geq 45$ years \\
2. Willing and able to provide written informed consent \\
3. Chest pain or chest discomfort main reason for visit \\
4. Willing and able to undergo complete study protocol
\end{tabular}

Exclusion criteria

1. Any cardiac history*

2. Contra-indications to CCTA prior to inclusion

3. Contra-indications to CMR prior to inclusion

4. Contra-indications to adenosine (second- or third-degree AV-block, COPD or asthma with evidence of bronchospasm)

In- and exclusion criteria are assessed by both the cardiologist and research physician before enrollment through patient records and direct contact with the participant upon inclusion.

*Cardiac history includes congenital heart disease, former cardiac procedures (angioplasty, myocardial infarction, bypass surgery, heart valve surgery or intervention, implantable cardiac defibrillator and/or cardiac resynchronization therapy, radiofrequency ablation, left ventricular assist device, heart transplantation and pacemaker implantation) 


\section{Participant recruitment}

The treating cardiologist at the referring center invites eligible patients to participate in the study at the end of the regular care visit. Participants that show interest in the study are contacted by the executive researcher. If these individuals provide informed consent, meet all inclusion criteria and none of the exclusion criteria, they are enrolled and undergo the study procedure. The study procedure consists of a standardized questionnaire, venous blood collection for measurement of known cardiovascular biomarkers and for storage in a biobank for later biomarker studies, as well as imaging by both CCTA and stress CMR. If participants are already scheduled to undergo a clinically indicated CCTA, these results are obtained for study purposes and the CCTA won't be performed as part of the study procedure. The workflow of the participant recruitment and imaging protocol for both participants with and without clinically indicated CCTA is visualized in Figure 1.

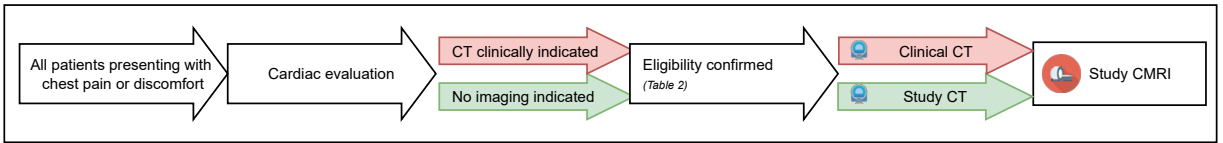

Figure 1. Workflow of patient recruitment and imaging protocol depending on regular clinical care path

\section{Sample size considerations}

Prior studies found CCTA-defined anatomical CAD to be present in $11.9 \%$, and CMRdefined myocardial ischemia in about $12.6 \%$ in low- to intermediate-risk patients with chest pain (19). Since we expect our population to be mostly low risk patients, we suspect the CAD prevalence to be at most $11 \%$. Based on our sample size calculation a number of 400 would be sufficient to estimate a prevalence of $11 \%$ with a precision of $3 \%$, using a 95\% confidence interval (https://epitools.ausvet.com.au/ oneproportion). Thus, the ARGUS study will run for three years, in which we expect to enroll 400 subjects and perform 400 CCTA and accompanying stress CMR scans.

\section{Regular care workup at the outpatient clinic}

Data collected during regular care includes medical history, physical examination, electrocardiography (ECG), laboratory blood measurements, exercise testing and transthoracic echocardiography. Co-morbidities and medication use are registered using a standardized questionnaire. All regular care measurements (including information on follow-up visits and performed external procedures) are recorded in the local electronic health records of the outpatient clinics and extracted for analysis in the study. 


\section{Case report form}

After obtaining informed consent, a study physician or trained research nurse fills out an extensive case report form with the participant, including information on symptoms (i.e. chest pain or chest discomfort specific characteristics such as location, character, duration, provoking or alleviating factors, such as relation to exercise, emotional stress, medication use or other symptoms such as dyspnea, palpitations or fatigue), cardiovascular risk factors, medical history, family cardiovascular history (first degree relatives with cardiovascular events), level of education and medication use. Additionally, pregnancy history and menopausal status are recorded for women.

\section{Blood sampling}

A venous blood sample $(75 \mathrm{~mL})$ from a peripheral vein is collected and processed according to our existing biobank protocol (20). In short, $2.5 \mathrm{~mL}$ ethylenediaminetetraacetic acid (EDTA) plasma tube is analyzed by the clinical chemistry department of the UMC Utrecht by an Abbott CELL-DYN Sapphire to obtain complete blood cell counts and additional raw blood cell characteristics. Furthermore, EDTA, citrate and sodium-heparin plasma and peripheral blood mononuclear cells (PBMC) isolated from EDTA blood are aliquoted and frozen at $-80 \mathrm{C}$ within 2 hours after the sample was collected. All samples are stored long-term at UMCU Central BioBank (CBB) for future studies, except for 1 aliquot of 700 ul which is transported to the Durrer Center for Cardiovascular research (Netherlands Heart Institute, Amsterdam, the Netherlands). As previously described, standard cardiac biomarker levels (B-type natriuretic peptide (BNP) and high-sensitivity troponin-I (hs-Tnl) (Abbott Diagnostics, Abbott Park, IL)) will be measured in plasma of all participants (20).

\section{Cardiac \& Coronary Computed Tomography Angiography (CCTA) Protocol}

Participants are asked to refrain from caffeine consumption for 4 hours and from food and nicotine for 2 hours prior to imaging. If the resting heart rate exceeds 60 beats per minute, heart rate lowering pre-medication can be prescribed based on clinical judgement of the treating cardiologist.

CCTA acquisitions will be performed using a 64-slice dual-layer spectral detector CT (SDCT) scanner (IQ on Spectral CT, Philips Healthcare, Best, The Netherlands) with $64 \times 0.625 \mathrm{~mm}$ collimation. In patients with a clinically scheduled CCTA in whom the scan could not be scheduled on the above-mentioned scanner, acquisitions will be performed according to local protocol, using either a 256-row multidetector scanner (Brilliance iCT, Philips Healthcare, Best, The Netherlands) or a 196-row CT-scanner (SOMATOM FORCE, Siemens Healthcare Diagnostics). Detailed scan parameters and acquisition protocol specifications are listed in Supplemental appendix 1. 
In participants with a heart rate above 60 beats per minute at time of the examination, intravenous beta-blocker will be administered in line with the Society of Cardiovascular CT guidelines (SCCT) (21). First, all participants will undergo coronary calcium scoring (CCS), which will be quantified by Agatston scores using commercially available software (HeartBeat CS, IntelliSpace Portal, Philips Healthcare, Best, The Netherlands) (22). Acquisitions will be made using a prospectively ECG-triggered protocol at the $78 \%$ of the R-R interval at $120 \mathrm{kVp}$ and $40 \mathrm{mAs}$. All acquisitions will be performed according to the recent SCCT guidelines (21). All images will be analyzed using a dedicated workstation (IntelliSpace Portal, Philips Healthcare, Best, The Netherlands). Subsequently, a CCTA scan will be acquired using the contrast agent $300 \mathrm{mg} \mathrm{I/mL}$ lopromide (Ultravist 300, Bayer Healthcare, Berlin, Germany) administered at a flow rate of $5.5 \mathrm{~mL} / \mathrm{s}(<80 \mathrm{~kg}$ ) or $6.0 \mathrm{~mL} / \mathrm{s}(\geq 80 \mathrm{~kg})$. Prior to the examination sublingual nitroglycerin $0.4 \mathrm{mg}$ will be administered in all participants. After contrast injection the intravenous line will be flushed using saline at the same flow rate. Seven seconds after the threshold of 200 Hounsfield Units (HU) is reached in the descending aorta the CCTA scan is acquired (prospectively ECG-triggered, $78 \%$ of the R-R interval at $120 \mathrm{kVp}$ and $150 \mathrm{mAs})$.

\section{Image Analysis}

Following SCCT guidelines all images will be assessed on image quality, plaque characteristics and degree of stenosis by the local cardiovascular radiologists (21). To provide a standardized assessment of CCTA, the Coronary Artery Disease - Reporting and Data system (CADRADS) classification will be reported (23). The estimated radiation dose for the CCTA examination is $5.7 \mathrm{mSv}$.

\section{Adenosine Stress Perfusion CMR \\ Protocol}

Stress CMR imaging will be performed on 1.5 or 3T scanners (Ingenia, Software release 5, Philips Healthcare, Best, The Netherlands) following a standardized protocol (Supplemental appendix 2). Twenty-four hours before all participants will be asked to avoid caffeine. First, native T1-weighted images will be acquired. Afterwards, stress and rest myocardial perfusion imaging will be performed during the first pass of gadobutrol (Gadovist ${ }^{\circledR}$, Bayer, Germany). Vasodilator stress will be achieved by adenosine infusion at $140 \mathrm{mcg} / \mathrm{kg} / \mathrm{min}$ for 4 minutes. Rest perfusion imaging will be acquired at least one minute after ending of adenosine infusion. Subsequently, cardiac function, post-contrast T1-weighted and viability imaging will be performed. The total duration will be approximately 50 minutes. 


\section{Image analysis}

All images will be analyzed by the local supervising CMR radiologists per regular clinical practice in accordance with the Society for Cardiovascular Magnetic Resonance (SCMR) guidelines (17). They will assess image quality, and report data on native- and post-contrast T1-values, quantitative functional analysis regarding end-diastolic and end-systolic volumes, ejection fraction, evidence of regional wall motion abnormalities (a-, hypo- or dyskinetic). Left ventricle strain imaging will be performed in long-axis cine images using Medis Suite MR software (Medis Medical Imaging Systems BV, Leiden). Subsequently, analysis of significant, not significant or no defect in perfusion at rest and stress, presence of scar tissue and transmurality based on the AHA/ACC 17 segment model will be reported (24). A perfusion defect is scored as a significant perfusion defect if ischemia is present in at least 2 segments and defined according to transmurality and persistence of the defect in accordance with the SCMR guidelines (17).

\section{Definition of coronary vascular disease}

The primary aim of the study is to provide insight into the prevalence of anatomical and functional coronary vascular disease. Coronary vascular disease is defined as either stress CMR-detected functional and/or CCTA-detected anatomical coronary vascular disease. Participants will be classified as positive or negative for any coronary vascular disease by expert panel consensus. The panel of experts comprises a cardiovascular radiologist and two cardiologists. The panel reviews all available data to reach a consensus whether coronary vascular disease is likely to be the underlying cause of the chest pain or discomfort (Figure 2). ESC guidelines for Chronic Coronary Syndromes are followed (25). First, the panel assesses the symptoms and signs to classify type of angina according to the traditional classification of suspected anginal symptoms (typical, atypical or non-anginal chest pain). Second, the general condition, risk factors, comorbidities and basic testing results collected during regular care are interpreted by the panel. Based on these regular care data the expert panel determines whether a cardiac cause is present (yes, no, possible) and, if so, whether they suspect the cause to be of macro- or microvascular or vasospastic origin. Following provision of the imaging results in combination with cardiac biomarker levels (BNP and hs-Tnl) the expert panel re-evaluates their decision. The workflow of the data collection and subsequent diagnostic approach by the expert panel is visualized in Figure 2.

The imaging results for both functional and anatomical coronary vascular disease will be documented separately and provided to the expert panel as follows: CCTA-detected anatomical coronary vascular disease based on the CADRADS-score assigned by the 


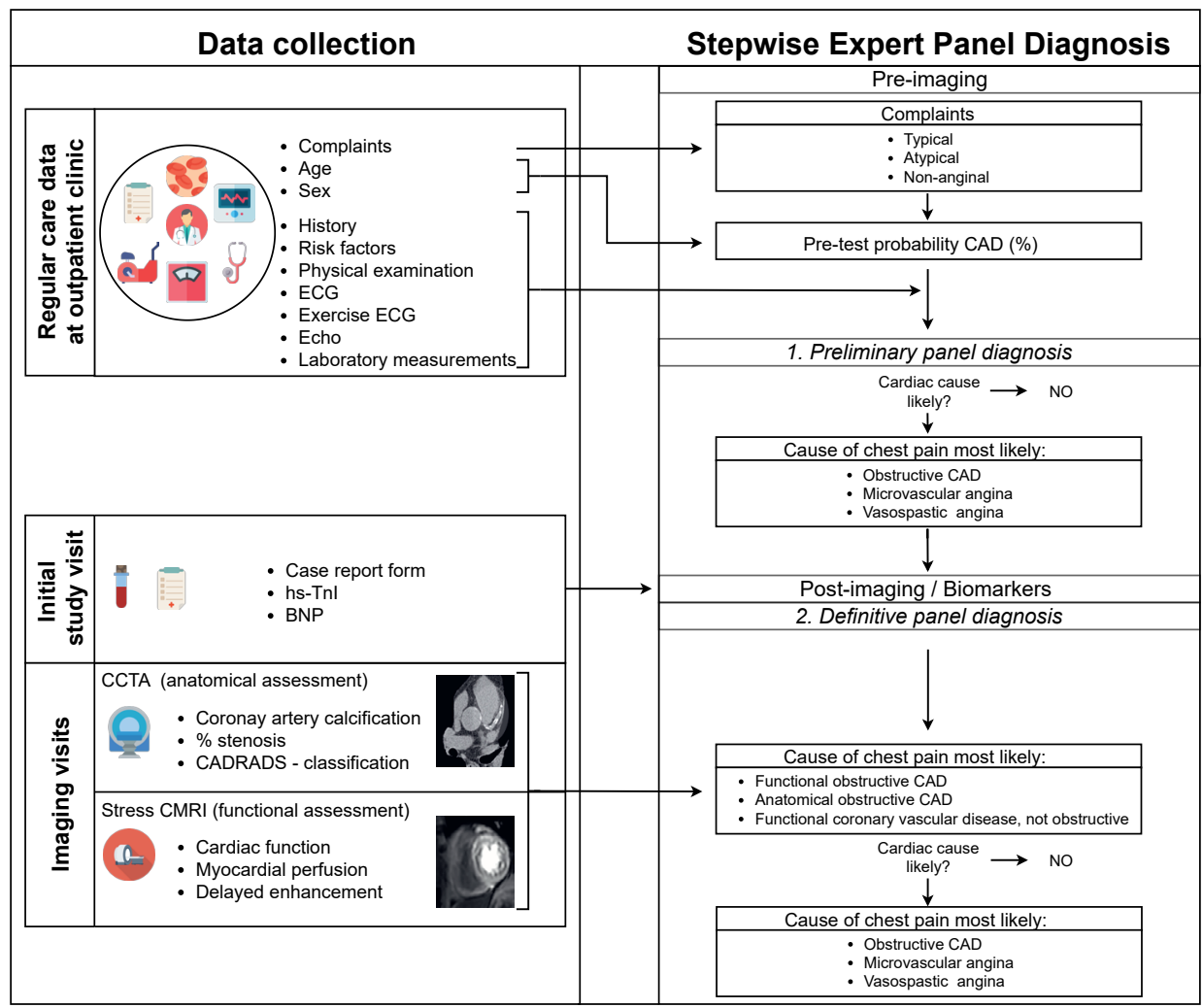

Figure 2. Workflow of data collection and implementation in the expert panel diagnosis

BNP, b-type natriuretic peptide; CAD, coronary artery disease; CADRADS, Coronary Artery Disease - Reporting and Data System; CCTA, cardiac computed tomography angiography; CMR, cardiac magnetic resonance; hs-Tnl, High-Sensitivity Troponin I

radiologist and stress CMR-detected functional coronary vascular disease based on visual assessment of presence or absence of myocardial ischemia by the radiologist (Table 2) (23).

\section{Long term follow-up}

By linkage with regional (Julius General Practitioners' Network) and national registries (i.e. Statistics Netherlands), all participants will be followed for consumption of medical care, occurrence of cardiac events, and all-cause and cause-specific mortality $(26,27)$. 
Table 2. Classification of imaging results

1. Assessment of anatomical CAD on CCTA

\begin{tabular}{lll}
\hline \multirow{2}{*}{$\begin{array}{ll}\text { Stenosis } \\
\text { CADRADS classification }\end{array}$} & Non-significant & Significant \\
\cline { 2 - 3 } 2. Assessment of functional CAD on CMR & $\geq 50 \%$ \\
\hline & $0 / 1 / 2$ & $3 / 4 / 5$ \\
\hline \multirow{2}{*}{ Myocardial ischemia } & Non-significant & Significant \\
\cline { 2 - 3 } & No & Yes \\
\hline
\end{tabular}

CCTA, cardiac computed tomography angiography; CAD, coronary artery disease; CADRADS, Coronary Artery Disease-Reporting and Data System, CMR, cardiac magnetic resonance

\section{Statistical analyses}

Descriptive data will be stratified by sex and presented as mean standard deviation (SD), or as medians with corresponding interquartile range if not normally distributed for continuous variables and numbers (with \%) for categorical variables. The prevalence of coronary vascular disease will be expressed as mean with a $95 \%$ confidence interval. Multivariable logistic regression will be used to analyze the association of various determinants at baseline with prevalent coronary vascular disease and the association between various anatomical and functional parameters at baseline. All analyses will be adjusted for appropriate a priori selected confounders (age, sex, medical history, comorbidities and cardiovascular risk factors). For prognostic studies, Cox proportional-hazards regression analysis will be used. All analyses will be performed both in the whole study population as well as stratified by sex.

The collected data is part of the development of a decision support tool. By means of machine learning techniques an algorithm will be trained to identify patterns between input data and outcome variables. Based on clinical data and extensive blood-based information as input variables the outcomes of the CCTA and CMR scans will be predicted in terms of the presence or absence of functional/anatomic coronary vascular disease. ARGUS will function as the clinical validation study for the developed algorithm.

\section{Study status}

ARGUS is actively recruiting participants. The number of participants recruited at the time of submission is 92 . Complete data of 70 participants is available, of whom $10.9 \%$ (5 women) were diagnosed with CMR-detected functional coronary vascular disease and $16.4 \%$ (13 women) with CCTA-detected anatomical coronary vascular disease. The current inclusion rate ( 5 participants per week) is sufficient to reach the goal of 400 included participants at the end of the inclusion period of 3 years. 


\section{Patient and public involvement}

Participants from the outpatient clinic CCN were involved in the design of the study and evaluation of the study protocol. Participants receive information on the study at summary level during the enrollment and after the study will be completed and are asked to participate in evaluation of the study.

\section{Ethics and dissemination}

The study protocol was approved by the Institutional Review Board of the University Medical Center Utrecht (NL_57077.041.16). The study is conducted according to the principles of the Declaration of Helsinki and in accordance with the Medical Research Involving Human Subjects Act (WMO). The study does not interfere with regular patient care, and all patients will be treated at the discretion of their cardiologist or general practitioner according to the appropriate guidelines. All participants give written informed consent for the study procedure. Results will be disseminated through national and international conferences and in peer-reviewed journals of cardiovascular disease.

\section{Funding}

The original investigator-initiated project HELPFul received funding from the Dutch Heart Foundation 2013 T084 (Queen of Hearts). The current ARGUS study using CMR and CCTA is supported by funding from CVON 2017-22 ARGUS.

\section{Acknowledgements}

We sincerely thank ms. Jonne Emanuel-Hos and Gideon Valstar for their outstanding efforts in and support to the HELPFul-ARGUS study. We would like to thank Evelyn Velema, Merel Schurink and Marja Maat-Leersum for their contribution to the recruitment of participants and their aid in validating and processing the raw data. We also thank Danny Elbersen, Sander van de Weg, Nanique Tulkens, Naomi Parr, Hemse Al-Khamisi, Lianne Granneman and Thuur Peeters for workup of the biobank samples. We thank Suzan Kemper-Bongers and the UPOD team for extracting and combining the regular care data for analysis. We thank all people involved from HeartLife Klinieken Utrecht and the Cardiology Center of the Netherlands and specifically the team at Cardiology Center Utrecht. We sincerely thank all people involved from the Radiology department of the UMC Utrecht for their efforts to perform both CCTA en CMR imaging in this study. A special thanks to all patients that participated in the study. 


\section{REFERENCES}

1. Hoorweg BB, Willemsen RT, Cleef LE, Boogaerts T, Buntinx F, Glatz JF, et al. Frequency of chest pain in primary care, diagnostic tests performed and final diagnoses. Heart. 2017;103(21):1727-32.

2. Conroy RM, Pyörälä K, Fitzgerald Ae, Sans S, Menotti A, De Backer G, et al. Estimation of ten-year risk of fatal cardiovascular disease in Europe: the SCORE project. European heart journal. 2003;24(11):987-1003.

3. Wilson PW, D'Agostino RB, Levy D, Belanger AM, Silbershatz H, Kannel WB. Prediction of coronary heart disease using risk factor categories. Circulation. 1998;97(18):1837-47.

4. Foldyna B, Udelson JE, Karády J, Banerji D, Lu MT, Mayrhofer T, et al. Pretest probability for patients with suspected obstructive coronary artery disease: re-evaluating Diamond-Forrester for the contemporary era and clinical implications: insights from the PROMISE trial. European Heart Journal-Cardiovascular Imaging. 2019;20(5):574-81.

5. Genders TS, Steyerberg EW, Alkadhi H, Leschka S, Desbiolles L, Nieman K, et al. A clinical prediction rule for the diagnosis of coronary artery disease: validation, updating, and extension. European heart journal. 2011;32(11):131630.

6. Truong QA, Rinehart S, Abbara S, Achenbach S, Berman DS, Bullock-Palmer R, et al. Coronary computed tomographic imaging in women: an expert consensus statement from the Society of Cardiovascular Computed Tomography. Journal of Cardiovascular Computed Tomography. 2018;12(6):451-66.

7. Jespersen L, Hvelplund A, Abildstrøm SZ, Pedersen F, Galatius S, Madsen JK, et al. Stable angina pectoris with no obstructive coronary artery disease is associated with increased risks of major adverse cardiovascular events. European heart journal. 2012;33(6):734-44.

8. Johnston N, Schenck-Gustafsson K, Lagerqvist B. Are we using cardiovascular medications and coronary angiography appropriately in men and women with chest pain? European heart journal. 2011;32(11):1331-6.

9. Sharaf B, Wood T, Shaw L, Johnson BD, Kelsey S, Anderson RD, et al. Adverse outcomes among women presenting with signs and symptoms of ischemia and no obstructive coronary artery disease: Findings from the National Heart, Lung, and Blood Institute-sponsored Women's Ischemia Syndrome Evaluation (WISE) angiographic core laboratory. American heart journal. 2013;166(1):134-41.

10. Sara JD, Widmer RJ, Matsuzawa Y, Lennon RJ, Lerman LO, Lerman A. Prevalence of coronary microvascular dysfunction among patients with chest pain and nonobstructive coronary artery disease. JACC: Cardiovascular Interventions. 2015;8(11):1445-53.

11. Wang ZJ, Zhang LL, Elmariah S, Han HY, Zhou YJ, editors. Prevalence and prognosis of nonobstructive coronary artery disease in patients undergoing coronary angiography or coronary computed tomography angiography: a meta-analysis. Mayo Clinic Proceedings; 2017: Elsevier.

12. Barr PR, Harrison W, Smyth D, Flynn C, Lee M, Kerr AJ. Myocardial infarction without obstructive coronary artery disease is not a benign condition (ANZACS-QI 10). Heart, Lung and Circulation. 2018;27(2):165-74.

13. Shaw LJ, Bugiardini R, Merz CNB. Women and ischemic heart disease: evolving knowledge. Journal of the American College of Cardiology. 2009;54(17):1561-75.

14. Camici PG, Crea F. Coronary microvascular dysfunction. New England Journal of Medicine. 2007;356(8):830-40.

15. Ong P, Safdar B, Seitz A, Hubert A, Beltrame J, Prescott E. Diagnosis of coronary microvascular dysfunction in the clinic. Cardiovascular Research. 2020.

16. Mathew RC, Bourque JM, Salerno M, Kramer CM. Cardiovascular Imaging Techniques to Assess Microvascular Dysfunction. JACC: Cardiovascular Imaging. 2019.

17. Kramer CM, Barkhausen J, Bucciarelli-Ducci C, Flamm SD, Kim RJ, Nagel E. Standardized cardiovascular magnetic resonance imaging (CMR) protocols: 2020 update. Journal of Cardiovascular Magnetic Resonance. 2020;22(1):118.

18. Knuuti J, Ballo H, Juarez-Orozco LE, Saraste A, Kolh P, Rutjes AWS, et al. The performance of non-invasive tests to rule-in and rule-out significant coronary artery stenosis in patients with stable angina: a meta-analysis focused on post-test disease probability. European heart journal. 2018;39(35):3322-30.

19. Hoffmann U, Ferencik M, Udelson JE, Picard MH, Truong QA, Patel MR, et al. Prognostic value of noninvasive cardiovascular testing in patients with stable chest pain: insights from the PROMISE trial (Prospective Multicenter Imaging Study for Evaluation of Chest Pain). Circulation. 2017;135(24):2320-32.

20. Valstar GB, Bots SH, Groepenhoff F, Gohar A, Rutten FH, Leiner T, et al. Discovery of biomarkers for the presence and progression of left ventricular diastolic dysfunction and heart failure with preserved ejection fraction in patients at risk for cardiovascular disease: rationale and design of the helpful case-cohort study in a Dutch cardiology outpatient clinic. BMJ open. 2019;9(6):e028408. 
21. Abbara S, Blanke P, Maroules CD, Cheezum M, Choi AD, Han BK, et al. SCCT guidelines for the performance and acquisition of coronary computed tomographic angiography: A report of the society of Cardiovascular Computed Tomography Guidelines Committee: Endorsed by the North American Society for Cardiovascular Imaging (NASCI). Journal of cardiovascular computed tomography. 2016;10(6):435-49.

22. van der Bijl N, Joemai RM, Geleijns J, Bax JJ, Schuijf JD, de Roos A, et al. Assessment of Agatston coronary artery calcium score using contrast-enhanced CT coronary angiography. American Journal of Roentgenology. 2010;195(6):1299-305.

23. Cury RC, Abbara S, Achenbach S, Agatston A, Berman DS, Budoff MJ, et al. CAD-RADSTM coronary artery disease-reporting and data system. An expert consensus document of the Society of Cardiovascular Computed Tomography (SCCT), the American College of Radiology (ACR) and the North American Society for Cardiovascular Imaging (NASCI). Endorsed by the American College of Cardiology. Journal of cardiovascular computed tomography. 2016;10(4):269-81.

24. Cerqueira MD, Weissman NJ, Dilsizian V, Jacobs AK, et al. Standardized myocardial segmentation and nomenclature for tomographic imaging of the heart: a statement for healthcare professionals from the Cardiac Imaging Committee of the Council on Clinical Cardiology of the American Heart Association. Circulation. 2002;105(4):53942.

25. Knuuti J, Wijns W, Saraste A, Capodanno D, Barbato E, Funck-Brentano C, et al. 2019 ESC Guidelines for the diagnosis and management of chronic coronary syndromes: the Task Force for the diagnosis and management of chronic coronary syndromes of the European Society of Cardiology (ESC). European heart journal. 2020;41(3):407-77.

26. Sollie A, Roskam J, Sijmons RH, Numans ME, Helsper CW. Do GPs know their patients with cancer? Assessing the quality of cancer registration in Dutch primary care: a cross-sectional validation study. BMJ open. 2016;6(9).

27. Reitsma J, Kardaun J, Gevers E, Bonsel G. Possibilities for anonymous follow-up studies of patients in Dutch national medical registrations using the Municipal Population Register: a pilot study. Nederlands tijdschrift voor geneeskunde. 2003;147(46):2286-90. 


\section{SUPPLEMENTARY MATERIAL}

\section{Supplemental appendix 1.}

\section{Cardiac \& Coronary Computed Tomography Angiography specifications}

\begin{tabular}{|c|c|c|c|c|c|c|}
\hline Scanner & \multicolumn{2}{|c|}{$\begin{array}{l}\text { Philips } \\
\text { IQon }\end{array}$} & \multicolumn{2}{|l|}{$\begin{array}{l}\text { Philips } \\
\text { Brilliance iCT }\end{array}$} & \multicolumn{2}{|c|}{$\begin{array}{l}\text { Siemens } \\
\text { SOMATOM FORCE }\end{array}$} \\
\hline & \multicolumn{2}{|c|}{ 64-slice } & \multicolumn{2}{|l|}{ 256-slice } & \multicolumn{2}{|c|}{$384(2 \times 192)$ slice } \\
\hline \multirow[t]{2}{*}{ Scan direction } & \multicolumn{2}{|c|}{ cranio-caudal } & \multicolumn{2}{|l|}{ cranio-caudal } & \multicolumn{2}{|c|}{ cranio-caudal } \\
\hline & \multicolumn{2}{|c|}{$\begin{array}{l}\text { Prospective step-and-shoot } \\
\text { (75\% RR-interval) }\end{array}$} & \multicolumn{2}{|c|}{$\begin{array}{l}\text { Prospective step-and-shoot } \\
\text { ( } 75 \% \text { RR-interval) }\end{array}$} & \multicolumn{2}{|c|}{$\begin{array}{l}\text { Prospective step-and-shoot } \\
\text { or turboflash ( } 75 \% \\
\text { RR-interval) }\end{array}$} \\
\hline Collimation (mm) & \multicolumn{2}{|c|}{$64 \times 0.625$} & \multicolumn{2}{|l|}{$128 \times 0.625$} & \multicolumn{2}{|c|}{$192 \times 0.6$} \\
\hline \multirow{2}{*}{ Increment } & \multicolumn{2}{|l|}{0.5} & \multicolumn{2}{|l|}{0.45} & \multicolumn{2}{|l|}{0.5} \\
\hline & CCS & CTA & CCS & CTA & CCS & CTA \\
\hline$k V p$ & 120 & 120 & 120 & $\begin{array}{l}100(<80 \mathrm{~kg}) \\
120(\geq 80 \mathrm{~kg})\end{array}$ & 120 & 100 \\
\hline mAs & $40^{\mathrm{a}}$ & $150^{\mathrm{a}}$ & $\begin{array}{l}40^{\mathrm{a}}(<80 \mathrm{~kg}) \\
50^{\mathrm{a}}(\geq 80 \mathrm{~kg})\end{array}$ & $210^{a}$ & $70 / 47$ & $350^{\mathrm{a}}$ \\
\hline Pitch & - & -- & - & - & 3.2 & 3.2 \\
\hline Rotatietijd & 0,5 & 0,27 & & 0.27 & 0.25 & 0.25 \\
\hline Contrast flowrate & - & $\begin{array}{l}5.5 \mathrm{~mL} / \mathrm{s}(<80 \mathrm{~kg}) \\
6.0 \mathrm{~mL} / \mathrm{s}(\geq 80 \mathrm{~kg})\end{array}$ & - & $\begin{array}{l}6.0 \mathrm{~mL} / \mathrm{s}(<80 \mathrm{~kg}) \\
6.7 \mathrm{~mL} / \mathrm{s}(\geq 80 \mathrm{~kg})\end{array}$ & - & $\begin{array}{l}6.0 \mathrm{~mL} / \mathrm{s}(<80 \mathrm{~kg}), \\
6.7 \mathrm{~mL} / \mathrm{s}(\geq 80 \mathrm{~kg})\end{array}$ \\
\hline $\begin{array}{l}\text { Treshold for CCTA } \\
\text { scan }\end{array}$ & & $150 \mathrm{HU}$ & & $130 \mathrm{HU}$ & - & $\begin{array}{l}\text { Peak } \\
\text { enhancement* }\end{array}$ \\
\hline $\begin{array}{l}\text { Post-treshold delay } \\
\text { (seconds) }\end{array}$ & - & 7 & - & 7 & - & 12 \\
\hline \multicolumn{7}{|l|}{ Reconstr. } \\
\hline Axial & $3 / 2$ & $3 / 3$ & $3 / 3$ & $3 / 2$ & $3 / 3$ & $3 / 3$ \\
\hline Coronal & & $3 / 2$ & & $3 / 2$ & & $3 / 2$ \\
\hline Sagittal & & $3 / 2$ & & $3 / 2$ & & $3 / 2$ \\
\hline
\end{tabular}

Abbreviations: CCS, coronary calcium scoring; CTA, coronary tomography angiography, HU, Hounsfield Units; ${ }^{\text {Reference }} \mathrm{mA}$, bEffective $\mathrm{mA}$, "Based on test bolus tracking 


\section{Supplemental appendix 2.}

\section{Adenosine Stress Perfusion Cardiac Magnetic Resonance (CMR) imaging protocol}

CMR imaging will be performed on 1.5 or 3 T scanners (Ingenia, Philips Healthcare, Best, The Netherlands) with use of a 28-element phased array surface coil. First cine images in the 4-, 3- and 2-chamber and short axis orientations will be acquired during mild expiration and ECG-triggering using a balanced turbo field echo sequence followed by basal, midventricular and apical pre contrast T1 weighted short axis slices. Rest and stress perfusion imaging will be performed. Maximal hyperemia for stress perfusion will be achieved by adenosine infusion at $140 \mathrm{mcg} / \mathrm{kg} / \mathrm{min}$ for 4 minutes. 24 hours before CMR imaging all participants will be asked to avoid caffeine and xanthine-containing foods. If a contra-indication for adenosine is present on the day of the CMR examination or participants did not abstain from caffeine for the past 24 hours, maximal hyperemia will be achieved by intravenous infusion of regadenoson (Rapiscan, 400 mcg, Astellas Pharma, Leiden, The Netherlands) and rest perfusion imaging will not be performed. After three minutes of adenosine infusion, stress perfusion images will be obtained at basal, midventricular, apical levels in the short axis as well as 4-chamber orientations during the first pass of $0.05 \mathrm{ml} / \mathrm{kg}$ (3T) or 0.1 $\mathrm{ml} / \mathrm{kg}\left(1.5 \mathrm{~T}\right.$ ) of gadobutrol (Gadovist ${ }^{\circledR}$, Bayer, Germany) injected at a flow rate of $4 \mathrm{mls} / \mathrm{s}$. Rest perfusion imaging will be acquired at least one minute after ending of adenosine infusion. Late gadolinium enhancement imaging will be carried out at least 10 minutes after the second bolus of the contrast agent. After assessment of the optimal inversion time ( $\mathrm{TI}$ ) viability, delayed enhancement viability imaging will be performed in the 2- and 4- chamber short axis view using an inversion recovery turbo gradient echo sequence. Afterwards delayed enhancement will be assessed using 4-chamber phase sensitive inversion recovery. At last post-contrast T1 weighted images in the basal, midventricular and apical short slices will be acquired. The total duration of the CMR imaging protocol will be approximately 50 minutes.

\begin{tabular}{|c|c|c|c|c|c|c|c|c|c|c|c|c|}
\hline \multirow{2}{*}{$\begin{array}{l}\text { MRI-sequence } \\
\text { Field strength }\end{array}$} & \multicolumn{2}{|c|}{ Looklocker } & \multicolumn{2}{|c|}{$\begin{array}{l}\text { Rest and stress } \\
\text { perfusion }\end{array}$} & \multicolumn{2}{|c|}{$\begin{array}{l}\text { SBTFE } \\
\text { 2-, 3- and 4- } \\
\text { chamber }\end{array}$} & \multicolumn{2}{|c|}{ T1 weighted } & \multicolumn{2}{|c|}{$\begin{array}{l}\text { Viability } \\
\text { 2- chamber }\end{array}$} & \multicolumn{2}{|c|}{$\begin{array}{l}\text { Viability } \\
\text { 4- chamber }\end{array}$} \\
\hline & $1.5 \mathrm{~T}$ & $3 T$ & $1.5 \mathrm{~T}$ & $3 \mathrm{~T}$ & $1.5 \mathrm{~T}$ & $3 \mathrm{~T}$ & $1.5 \mathrm{~T}$ & $3 \mathrm{~T}$ & $1.5 \mathrm{~T}$ & $3 T$ & $1.5 \mathrm{~T}$ & $3 \mathrm{~T}$ \\
\hline $\begin{array}{l}\text { Flip angle } \\
\text { (degrees) }\end{array}$ & 12 & 7 & 50 & 15 & 60 & 45 & 35 & 20 & 15 & 25 & 15 & 25 \\
\hline $\begin{array}{l}\text { Act. TR/TE } \\
(\mathrm{ms})\end{array}$ & $\begin{array}{l}8.0 / \\
3.1\end{array}$ & $\begin{array}{l}8.0 / \\
3.5\end{array}$ & $\begin{array}{l}2.6 / \\
1.31\end{array}$ & $\begin{array}{l}3.7 / \\
1.78\end{array}$ & $\begin{array}{l}3.4 / \\
1.7\end{array}$ & $\begin{array}{l}2.8 / \\
1.4\end{array}$ & $\begin{array}{l}1.99 / \\
0.88\end{array}$ & $\begin{array}{l}2.2 / \\
1.02\end{array}$ & $\begin{array}{l}3.6 / \\
1.15\end{array}$ & $\begin{array}{l}6.1 / \\
3.0\end{array}$ & $\begin{array}{l}3.5 / \\
1.15\end{array}$ & $\begin{array}{l}6.1 / \\
3.0\end{array}$ \\
\hline $\begin{array}{l}\text { Min. TI delay } \\
(\mathrm{ms})\end{array}$ & 15.2 & 13.9 & & & & & 117.9 & 135.4 & 118.3 & 92.5 & 110.2 & 92.4 \\
\hline
\end{tabular}

Abbreviations: $\mathrm{SBTFE}=$ balanced turbo field echo, $\mathrm{T}=\mathrm{Tesla}, \mathrm{TR}=$ repetition time, $\mathrm{TE}=$ echo time, $\mathrm{TI}=$ inversion time 


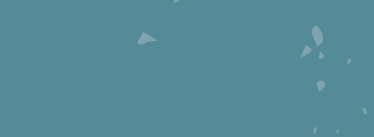

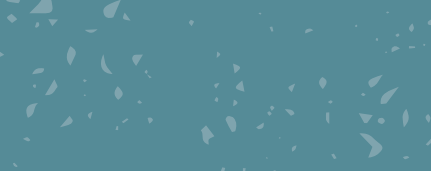

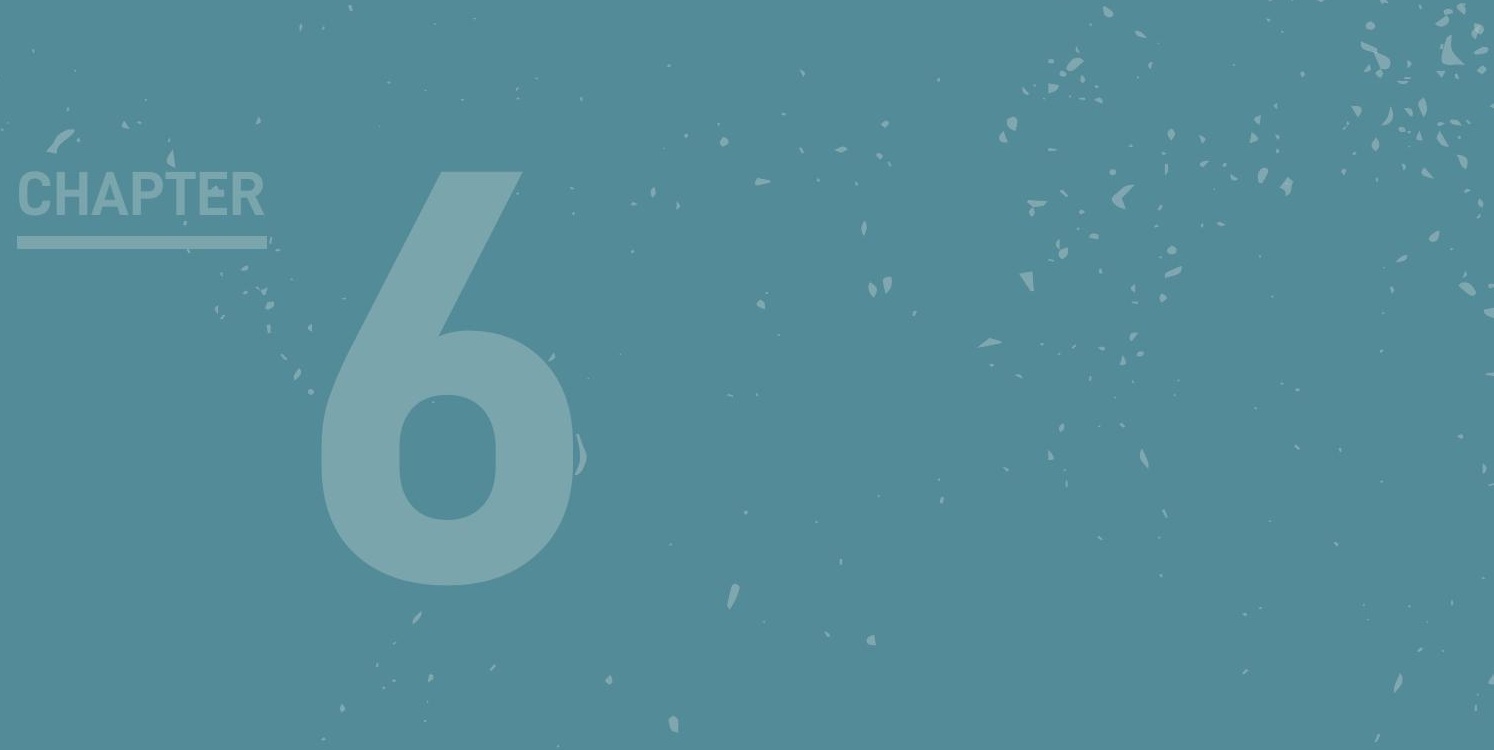

CHAPTTER 


\section{Women with higher grades of coronary stenoses have lower calcium scores than men}

In preparation 
Coronary artery calcium is highly prevalent in coronary artery disease (CAD) and predicts all-cause mortality (1). Therefore, the coronary artery calcium score (CACS) is widely used as risk marker in both women and men suspected of CAD. CACS is generally low in women and the diagnostic value of CACS to detect CAD is also lower in women than in men (2). This may be a consequence of coronary plaques more often being non-calcified or mixed in women, whereas in symptomatic men plaques are mainly calcified (3). To describe CAD severity beyond calcification, the Coronary Artery Disease Reporting and Data System (CAD-RADS) has been developed (4). CADRADS classifies CAD severity based on both stenosis degree and presence of (non-) calcified atherosclerotic plaque. The PROMISE trial showed that CAD-RADS is more powerful than CACS or traditional stenosis-based assessment in prediction of adverse events and mortality, and has clear added value (1). It may well be that CAD-RADS identifies high-risk non-calcified plaques that are more prevalent in women, but sexstratified data were not published. We hypothesize that the sex difference in plaque composition is reflected by a sex-specific relation between CACS and CAD severity, scored by CAD-RADS.

We studied the association between CACS and CAD-RADS (4) in 121 symptomatic men $(n=46)$ and women $(n=75)$. Included patients had no cardiac history, visited a Dutch outpatient cardiology clinic for the first time with chest discomfort, were suspected of CAD, and underwent coronary computed tomography angiography (CCTA) (5). The relation between CACS and CAD-RADS was assessed using sex-stratified Kendall's rank correlation analysis. CAD-RADS was categorized in clinically relevant categories of no (CAD-RADS 0), minor (CAD-RADS 1-2) and significant CAD (CAD-RADS $\geq 3$ ) (4). A two-sided $P<0.05$ was considered statistically significant. The study was approved by the Institutional Review Board of the University Medical Center Utrecht and all participants provided written informed consent.

Characteristics of the study population are displayed in Figure 1, panel A. Age was comparable between the sexes (a mean age of 60 (SD 10) years in women vs 62 (SD 8 ) years in men). Interestingly, women more often reported dyspnea in combination with chest discomfort (63\% in women vs 30\% in men). In women with chest discomfort, CACS was mostly absent (median CACS 0 [IQR: 0-55]). In those women with CAD-RADS $\geq 1$ ( $n=41(55 \%)$ ), which indicates the presence of coronary plaque, CACS was also low (median CACS 32 [IQR: 9-137]). In men, CACS was mostly present (median CACS 44 [IQR: 0 - 306]). Moreover, coronary plaque (CAD-RADS $\geq 1$ ) was present in 39 (85\%) men. In these men with coronary plaques, median CACS was 79 [IQR: 8 - 412]. Overall, the CAD severity by means of CAD-RADS was lower in women; CAD- RADS $\geq 3$ was present in $9 \%$ of women vs $35 \%$ of men. The association between 
CACS and CAD-RADS is visualized in Figure 1, panel $B$. In both sexes, CACS significantly increased with higher CAD-RADS scores (Kendall's rank correlation tau $=0.74$ in women, 0.45 in men, both $P<0.01$ ). Despite the significantly stronger positive association in women as compared with men $(P=0.008)$, CACS did not rise as strong with increasing CAD-RADS score in women as compared with men. In women with minor CAD (CAD-RADS 1 or 2) median CACS was 27 [IQR: 5 - 113]. In women with significant CAD (CAD-RADS $\geq 3$ ) median CACS was 195 [IQR: $129-265$ ]. In men the increase in CACS was steeper; from a median CACS of 0 [IQR: $0-0$ ] in no CAD to a median CACS of 45 [IQR: 8 -152] in minor CAD to a median CACS of 363 [IQR: $12-$ 1219] in significant CAD (CAD-RADS $\geq 3$ ).

In conclusion, the relation between the degree of CAD defined by CAD-RADS score and coronary calcification seems dependent on sex. We found that within similar coronary stenosis severity categories (as defined by CAD-RADS), women have lower CACS than men. In women with significant CAD, i.e. CAD-RADS $\geq 3$, median CACS is not even half the score of CACS observed in men. Our preliminary findings imply that women can have relatively high degrees of coronary stenoses with little calcification. Therefore, CAD-RADS could have added value on top of CACS, specifically in women, and warrants further investigation.

\begin{tabular}{|c|c|c|c|c|c|}
\hline & Women & Men & \multirow[t]{2}{*}{$3000-$} & \multirow{8}{*}{ - } & \\
\hline $\mathbf{N}$ & 75 & 46 & & & \\
\hline Age (mean (SD)) & $60(8)$ & $62(10)$ & \multirow[b]{4}{*}{2000} & & \\
\hline BMI (mean (SD)) & $26(5)$ & $27(3)$ & & & \\
\hline Smoker = yes, n (\%) & $5(7)$ & $1(2)$ & & & \\
\hline Smoker ever = yes, $\mathrm{n}(\%)$ & $36(51)$ & $26(59)$ & & & \\
\hline Hypertension = yes $\mathbf{n}(\%)$ & $31(41)$ & $24(52)$ & \multirow{4}{*}{ 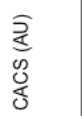 } & & \\
\hline Anti-hypertensive medications use $=$ yes, $n(\%)$ & $24(77)$ & $17(71)$ & & & \\
\hline Hypercholesterolemia = yes, $\mathbf{n}(\%)$ & $28(37)$ & $19(41)$ & & & \\
\hline Dyspnea = yes, $\mathrm{n}(\%)$ & $47(63)$ & $14(30)$ & & • & \\
\hline Family history of CVD = yes, $n(\%)$ & $59(79)$ & $28(61)$ & \multirow[t]{2}{*}{1000} & & • \\
\hline CACS (median [IQR]) & $0[0 ; 55]$ & $44[0 ; 306]$ & & • & \\
\hline \multicolumn{3}{|l|}{ CAD-RADS, n (\%) } & \multirow[b]{4}{*}{ B. } & & \\
\hline 0 & $34(45)$ & $7(15)$ & & & E \\
\hline $1-2$ & $34(45)$ & $23(50)$ & & & \\
\hline A. $^{3-5}$ & $7(9)$ & $16(35)$ & & Minor CAD & Sig CAD \\
\hline
\end{tabular}

Figure 1. A, Baseline characteristics of the study population per sex; B, CACS plotted against stenosis severity in men (blue) and women (pink)

BMI, body mass index; CVD, cardiovascular disease; CACS, coronary artery calcification score; CAD-RADS, Coronary Artery Disease Reporting and Data System; AU, Agatston Units; No CAD = CAD-RADS 0, no atherosclerosis or stenosis; Minor CAD $=$ CAD-RADS 1 or 2 , any stenosis $<50 \%$; Significant $C A D=C A D-R A D S \geq$ 3 , any stenosis $>50 \%$ 


\section{REFERENCES}

1. Bittner DO, Mayrhofer T, Budoff M, Szilveszter B, Foldyna B, Hallett TR, et al. Prognostic value of coronary CTA in stable chest pain: CAD-RADS, CAC, and cardiovascular events in PROMISE. Cardiovascular Imaging. 2020;13(7):1534-45.

2. Nakao YM, Miyamoto Y, Higashi M, Noguchi T, Ohishi M, Kubota I, et al. Sex differences in impact of coronary artery calcification to predict coronary artery disease. Heart. 2018;104(13):1118-24.

3. Plank F, Beyer C, Friedrich G, Wildauer M, Feuchtner G. Sex differences in coronary artery plaque composition detected by coronary computed tomography: quantitative and qualitative analysis. Netherlands Heart Journal. 2019;27(5):272-80.

4. Cury RC, Abbara S, Achenbach S, Agatston A, Berman DS, Budoff MJ, et al. Coronary Artery Disease-Reporting and Data System (CAD-RADS) An Expert Consensus Document of SCCT, ACR and NASCI: Endorsed by the ACC. JACC: cardiovascular imaging. 2016;9(9):1099-113.

5. Groepenhoff F, Eikendal AL, Bots SH, van Ommen A-M, Overmars L, Kapteijn D, et al. Cardiovascular imaging of women and men visiting the outpatient clinic with chest pain or discomfort: design and rationale of the ARGUS Study. BMJ open. 2020;10(12):e040712. 


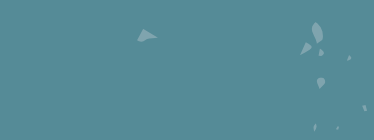

CEAPTER $\because \because \quad \because \quad \therefore \quad \therefore \quad \therefore$ 


\title{
Coronary calcification measures predict mortality in symptomatic women and men
}

\author{
Submitted
}

Groepenhoff $F^{*}$, Siegersma KR*, Eikendal ALM', op den Brouw WJ, Leiner T, Appelman $\mathrm{Y}$, Tulevski II, Somsen GA, Onland-Moret NC, Hofstra L*, den Ruijter HM* 


\section{ABSTRACT}

Coronary Artery Calcium Score (CACS) is a strong predictor for mortality in asymptomatic individuals. However, in patients with symptoms suspicious for cardiac disease data on the value of CACS is scarce, despite its wide use in this population. Sex, age and race/ethnic differences in atherosclerotic plaque composition and development might imply a need to use personalized percentiles instead of absolute CACS. To assess the prognostic value of absolute and sex-, age-, and race/ethnicityspecific percentiles of coronary artery calcification in symptomatic women and men we performed an observational study in 4985 symptomatic patients (2793 women, 56\%) visiting a diagnostic outpatient cardiology clinic who were referred for cardiac computed tomography (CT) to determine CACS. Data were linked to the databases of Statistics Netherlands for all-cause mortality data. Kaplan-Meier curves, multivariate Cox proportional hazards regression and concordance statistics were used to evaluate the prognostic value of CACS and sex-, age-, and race/ethnicityspecific percentiles. Women were older compared to men (60 vs. 59 years). Median CACS was 0 (IQR: 0-54) in women and 42 (IQR: 0-278) in men. After a median follow up of 4.4 years (IQR: 3.1 to 6.3 ), 116 (2.3\%; 53 women and 63 men) patients died. Sex-, age-, and race/ethnicity-specific percentiles did not perform better compared to absolute CACS (C-statistic $0.65,95 \%$ confidence interval [CI]: $0.57-0.73$, vs 0.66 , $95 \% \mathrm{Cl}: 0.58-0.74$, in women and $0.59,95 \% \mathrm{Cl}: 0.51-0.67$, vs $0.62,95 \% \mathrm{Cl}: 0.55-0.69$, in men, for the percentiles and absolute CACS, respectively). In conclusion, in symptomatic patients at cardiac outpatient clinics, absolute and sex-, age-, and race/ ethnicity-specific percentiles of CACS predict mortality equally well, in both women and men. Hence, there is no need to use personalized, sex-, age-, and race/ethnicityspecific, percentiles instead of absolute CACS. 


\section{INTRODUCTION}

In asymptomatic women and men, calcification of the coronary arteries is proven to be a strong predictor for mortality (1). Moreover, patients with no coronary artery calcification have a very low prevalence of obstructive coronary artery disease (CAD) and their long-term prognosis is relatively good (2). In individuals with symptoms suspicious for cardiac disease, coronary artery calcification is also widely used to assess mortality risk. However, its value in these symptomatic individuals is not well established (3). To evaluate the calcification of the coronary arteries and the presence of CAD, a non-contrast enhanced computed tomography (CT)-scan is recommended in symptomatic patients with an intermediate pre-test probability of CAD. This pretest probability is based on sex, age and type of complaints (4). Based on the results of this diagnostic assessment, the amount of coronary artery calcium is quantified with use of the coronary artery calcium score (CACS) by the Agatston method (5). CACS, as a continuous variable, is often categorized for clinical use in four different categories: no calcification (CACS = 0), minimal (CACS 1-100), moderate (CACS 101399) and severe calcification (CACS $\geq 400$ ).

Several studies demonstrated sex differences in the amount and type of atherosclerotic plaques. Symptomatic men mostly have calcified plaques while women predominantly have mixed or non-calcified plaques $(6,7)$. Therefore, use of absolute CACS to estimate mortality risk may lead to false reassurance in women with low CACS, as symptomatic women might have CAD caused by non-calcified plaques (8). Thus, CACS may have a different prognostic value in women compared to men. Moreover, the presence of coronary artery calcification also differs between ethnicities (9) and increases with increasing age (10). Therefore, the Multi-Ethnic Study of Atherosclerosis (MESA) reported sex-, age-, and race/ethnicity-specific percentiles for CACS in the general and asymptomatic population $(11,12)$. However, the question remains if these percentiles are a better discriminator of risk compared to absolute CACS in symptomatic patients in a real-world cardiology setting. Furthermore, the value of degree of stenosis for mortality prediction might differ in women compared to men, since non-calcified plaques, that are more prevalent in women $(6,7)$, are not reflected in the CACS.

To address these questions, we first studied the prognostic value of coronary artery calcification measures in a symptomatic population in a sex-stratified manner, as CACS is mainly assessed and used as a risk marker in these symptomatic women and men (13-15). Both absolute measures, as reflected by CACS, and sex-, age-, and race/ ethnicity-specific percentiles (11) were evaluated as measures to reflect the amount 
of calcification. Second, we evaluated whether degree of coronary stenosis at CT angiography increases the discriminative prognostic value when added to the model based on CACS.

\section{METHODS}

\section{Patient selection}

Individual patient data from electronic health records (CardioPortal ${ }^{\mathrm{TM}}$, Cardiology Centers of the Netherlands proprietary electronic health records, EHR) was retrieved from thirteen Dutch outpatient cardiology clinics (Cardiology Centers of the Netherlands, CCN) between April 2007 and February 2018. A detailed description of the population of this cohort has been previously published (16). The Medical Research Ethics Committee of the UMCU declared that the Medical Research Involving Human Subjects does not apply to this study. We analyzed patients that underwent cardiac CT as part of clinical care, resulting in a study population of 4985 women and men presenting at one of the outpatient cardiology clinics of CCN, aged between 45 and 85 years (Figure 1). Standardized workup was performed and documented for these patients, including information on cardiovascular risk factors (age, history of smoking, history of cardiovascular disease, presence of diabetes mellitus, hypertension, dyslipidemia, body mass index (BMI) and signs of heart failure).

\section{Calcium score and degree of stenosis assessment}

Cardiac CT was composed of a scan protocol to calculate CACS and a contrastenhanced protocol for coronary angiography. Results of the cardiac CT were reported in free text. This text was transformed into different features, e.g. CACS and degree of stenosis in one of the main coronary arteries. We determined sex-, age-, and race/ ethnicity-specific percentiles based on retrieved absolute CACS for all women and men using the previously reported percentile tables from the MESA-study (12), as these were not structurally documented in the EHR. As CACS is clinically used in different categories, we categorized CACS into the following groups: zero CACS, CACS 1 to 100 , CACS 101 to 400 and CACS $>400$. For sex-, age-, and race/ethnicity-specific percentiles the following categories were used: no CAC, $\leq 75$ th percentile, 75 th to 90th percentile, and >90th percentile. In 2715 (54\%) of these patients, degree of stenosis determined by CT angiography was also documented in free text. A comparison was made for baseline characteristics of the complete CT population in which CACS was reported and the CT population in whom degree of stenosis was additionally documented. The available CT angiography results were classified into grades of stenosis, namely $0 \%, 1-24 \%, 25-49 \%, 50-70 \%, 71-99 \%$ and $100 \%$ stenosis 


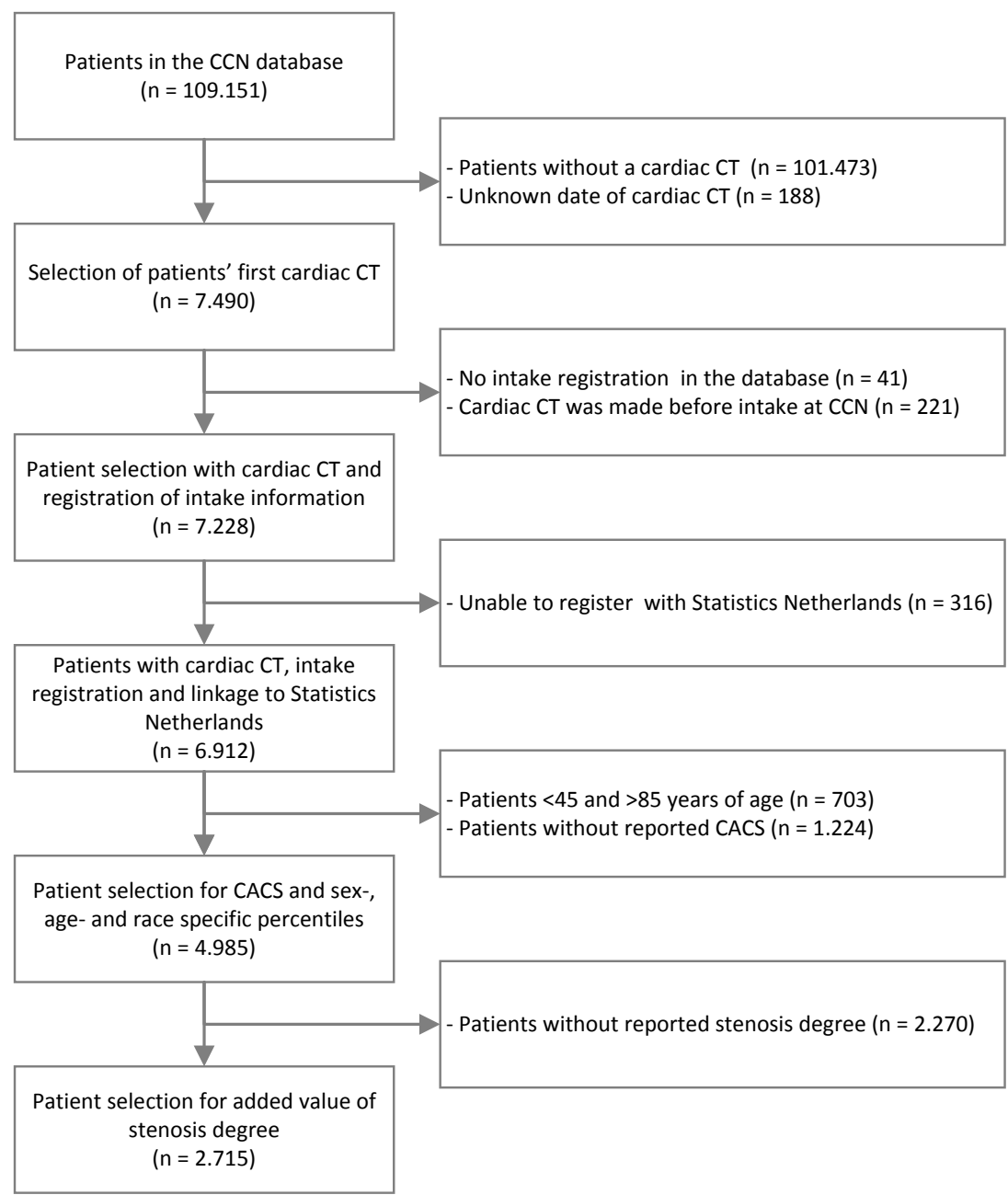

Figure 1. Patient selection for CACS and sex-, age- and race/ethnicity-specific analysis and for analysis of addition of stenosis degree

(17). The following textual terms indicating stenosis severity were used to classify the degree of stenosis if the absolute percentage was missing; for 1-24\% any, minimal, minor, for $25-49 \%$ partial, diffuse, mild, not-significant, non-significant, for $50 \%-70 \%$ important, clear, significant, intermediate, for 71-99\% severe, high grade and for $100 \%$ occlusion. As (high risk) plaque characteristics were irregularly and unstructured mentioned in free text, these features were not taken into account for analysis. 


\section{Outcome assessment}

Information on patients' country of origin, mortality and cause of death was obtained by linkage to the population registry of Statistics Netherlands. The results are based on calculations by UMC Utrecht using non-public microdata from Statistics Netherlands.

\section{Statistical analyses}

The baseline characteristics of the datasets were described as mean +/- standard deviation (SD) or median with interquartile range (IQR) when appropriate. Due to privacy constraints, numbers of individuals fewer than 10 are not allowed to be exported from Statistics Netherlands. In all tables, these are represented as ' $<10^{\prime}$ with the corresponding percentage. We estimated survival functions using Kaplan-Meier method. Cox proportional hazards regression analysis was performed to study the association between measures of coronary artery calcification (absolute CACS as calculated by the Agatston score and sex-, age-, and race/ethnicity-specific percentiles) and mortality. For the model based on the sex-, age-, and race/ethnicity-specific percentiles, a binary variable was added to indicate whether CACS was positive $(>0)$ at baseline. The addition of a variable to indicate the presence or absence of CACS is needed to correct for the possible discontinuity in the sex-, age-, and race/ethnicityspecific percentiles between individuals with a CACS of zero or any positive continuous CACS. We first constructed models including CACS or sex-, age-, and race/ethnicityspecific percentiles as continuous variables. Subsequently, we constructed models with the previously described categorized CACS and sex-, age-, and race/ethnicityspecific percentiles. The concordance statistic (C-statistic) with $95 \%$ confidence intervals $(\mathrm{Cl})$ was used to assess the ability of these models to discriminate patients at risk for mortality. A higher C-statistic indicates a better fit and higher prognostic power. Subsequently, we evaluated whether addition of degree of stenosis on top of absolute CACS improved the prognostic power of the Cox proportional hazards model. All analyses were performed stratified by sex. All statistical analyses were performed in $\mathrm{R}$ (version 4.0.2).

\section{Sensitivity analyses}

The race groups within the sex-, age-, and race/ethnicity-specific-percentiles as defined by the MESA-study (12) were not identical to patients' country of origin as documented in the population registry of the Statistics Netherlands. In our primary analyses we calculated sex-, age-, and race/ethnicity-specific percentiles based on the Caucasian race. In a sensitivity analysis we excluded all patients born outside Europe, using this individual's characteristic as a surrogate for a different race. 


\section{RESULTS}

\section{Baseline characteristics}

Baseline characteristics of the selected population are displayed in Table 1. Of the 4985 patients, 2793 (56\%) patients were women. On average, women were one year older compared to men (60 vs. 59 years of age). Other cardiovascular risk factors were similar between women and men. Both sexes primarily presented with chest pain or discomfort. Median CACS in women was 0 (IQR: 0-54), and 42 (IQR: 0-278) in men. CACS categories of $0,1-100,100-400$ and $>400$ were respectively present in 1387 (50\%), 899 (32\%), $340(12 \%)$ and $167(6 \%)$ of all women in this study. In men this distribution was 569 (26\%), 774 (35\%), 436 (20\%) and 413 (19\%), respectively. Higher CACS was associated with higher age and a higher prevalence of hypertension and dyslipidemia in both sexes (Supplementary Table 1).

Table 1. Baseline characteristics of study population per sex

\begin{tabular}{|c|c|c|c|}
\hline & Overall & Women & Men \\
\hline $\mathrm{n}$ & 4985 & 2793 & 2192 \\
\hline Age in years & $59(8)$ & $60(8)$ & $59(8)$ \\
\hline Body mass index & $27(5)$ & $27(5)$ & $27(4)$ \\
\hline Originated from Europe, $n$ (\%) & $4309(86)$ & $2411(86)$ & $1898(87)$ \\
\hline \multicolumn{4}{|l|}{ Complaints, n (\%) } \\
\hline Chest pain or discomfort & $2683(54)$ & $1585(57)$ & $1098(50)$ \\
\hline Dyspnea & $538(11)$ & $329(12)$ & $209(10)$ \\
\hline Fatigue & $172(4)$ & $85(3)$ & $87(4)$ \\
\hline Palpitations & $463(9)$ & $290(10)$ & $173(8)$ \\
\hline Collapse & $27(1)$ & $<10$ & $>10$ \\
\hline \multicolumn{4}{|l|}{ Smoking status, $\mathrm{n}(\%)$} \\
\hline Current & $1476(32)$ & $859(33)$ & $617(30)$ \\
\hline Former & $1650(36)$ & $875(34)$ & $775(38)$ \\
\hline Never & $1504(33)$ & $854(33)$ & $650(32)$ \\
\hline Diabetes Mellitus, n (\%) & $396(8)$ & $213(8)$ & $183(8)$ \\
\hline Hypertension, n (\%) & $1581(32)$ & $954(34)$ & $627(29)$ \\
\hline Dyslipidemia, n (\%) & $850(17)$ & $477(17)$ & $373(17)$ \\
\hline CACS score (median [IQR]) & $8[0,121]$ & $0[0,54]$ & $42[0,278]$ \\
\hline \multicolumn{4}{|l|}{ CACS catgory, $\mathrm{n}(\%)$} \\
\hline 0 & $1956(39)$ & $1387(50)$ & $569(26)$ \\
\hline $1-100$ & $1673(34)$ & $899(32)$ & $774(35)$ \\
\hline $101-400$ & $776(16)$ & $340(12)$ & $436(20)$ \\
\hline$>400$ & $580(12)$ & $167(6)$ & $413(19)$ \\
\hline \multicolumn{4}{|l|}{ Examinations during follow-up: } \\
\hline At least one CAG, $n(\%)$ & $812(16)$ & $309(11)$ & $503(23)$ \\
\hline At least one $\mathrm{PCl}$ or $\mathrm{CABG}, \mathrm{n}(\%)$ & $307(6)$ & $89(3)$ & $218(10)$ \\
\hline Years of follow up (median [IQR]) & $4.5[3.1,6.3]$ & $4.5[3.1,6.3]$ & $4.5[3.1,6.3]$ \\
\hline
\end{tabular}

CACS, coronary artery calcium score; $\mathrm{CAG}$, coronary angiography; $\mathrm{PCl}$, percutaneous coronary intervention; $\mathrm{CABG}$, coronary artery bypass graft 


\section{The association between coronary calcification and mortality}

After a median follow up of 4.5 years (IQR: 3-6), 116 (53 women and 63 men) patients died, of which 22 were attributed to cardiovascular mortality. Mortality rate was higher in women and men who had higher CACS (women: $1 \%, 2 \%, 4 \%$ and $<6 \%$, men: $<2 \%, 3 \%, 3 \%$ and $5 \%$, for, respectively CACS categories of $0,1-100,100-400$ and $>$ 400, Supplementary Table 1). Figure 2 and 3 show the survival over time per calcification category for women and men (absolute CACS in Figure 2, sex-, age-, and race/ethnicity-specific percentiles in Figure 3). Overall, women had a better survival. Higher levels of absolute CACS were associated with worse survival compared to low CACS levels. The association was also seen with higher sex-, age-, and race/ethnicityspecific percentiles, albeit less strong. However, differences were small.

Mortality rates, hazards ratios and C-statistics are displayed in Table 2 for both women and men. For continuous calcification measures, the discriminative ability of absolute CACS was moderate in both women and men (C-statistic: $0.66,95 \% \mathrm{Cl}: 0.58-0.74$ in women, and $0.62,95 \% \mathrm{Cl}: 0.55-0.69$, in men). The performance of the models on absolute CACS were similar to those based on sex-, age-, and race/ethnicity-specific percentiles ( $0.65,95 \% \mathrm{Cl}$ : 0.57-0.73 in women, 0.59 , 95\% Cl: 0.51-0.67 in men). Results were similar for the models that used categorical classifications of CACS and sex-, age-, and race/ethnicity-specific percentiles instead of continuous measures. The categorical CACS model showed a C-statistic of 0.65 (95\% Cl: 0.57-0.73) in women, and 0.60 (95\% Cl: 0.53-0.67) in men. The categorical sex-, age-, and race/ethnicityspecific percentile model had C-statistics for women and men of, respectively, 0.64 (95\% Cl: 0.57-0.73) and 0.61 (95\% Cl: 0.54-0.67).

The sensitivity analyses in which we excluded all patients in whom the country of birth was documented to be outside Europe ( $n=676,13.5 \%)$, as a surrogate for a different race, showed similar results. The European population comprised 2411 women and 1898 men. The C-statistic for absolute CACS was comparable to the sex, age- and race/ethnicity-specific percentiles (in women, $0.67,95 \% \mathrm{Cl}: 0.59-0.76$, vs $0.66,95 \% \mathrm{Cl}: 0.58-0.75$, and in men $0.64,95 \% \mathrm{Cl}: 0.55-0.72$, vs $0.59,95 \%-\mathrm{Cl}: 0.510 .67$ ). All results of the sensitivity analysis are displayed in Supplementary Table 2.

\section{Stenosis degree and the association between coronary calcification and mortality} Stenosis severity by CT angiography was documented for 1330 (60.7\%) men and 1385 (49.6\%) women. Baseline characteristics of these women and men are depicted in Table 3. Supplementary Table 3 shows the baseline characteristics of the CT population in which CACS was reported and the CT population in whom degree of stenosis was additionally documented. During a median follow-up of 5 years (IQR: 3-6 years), 46 


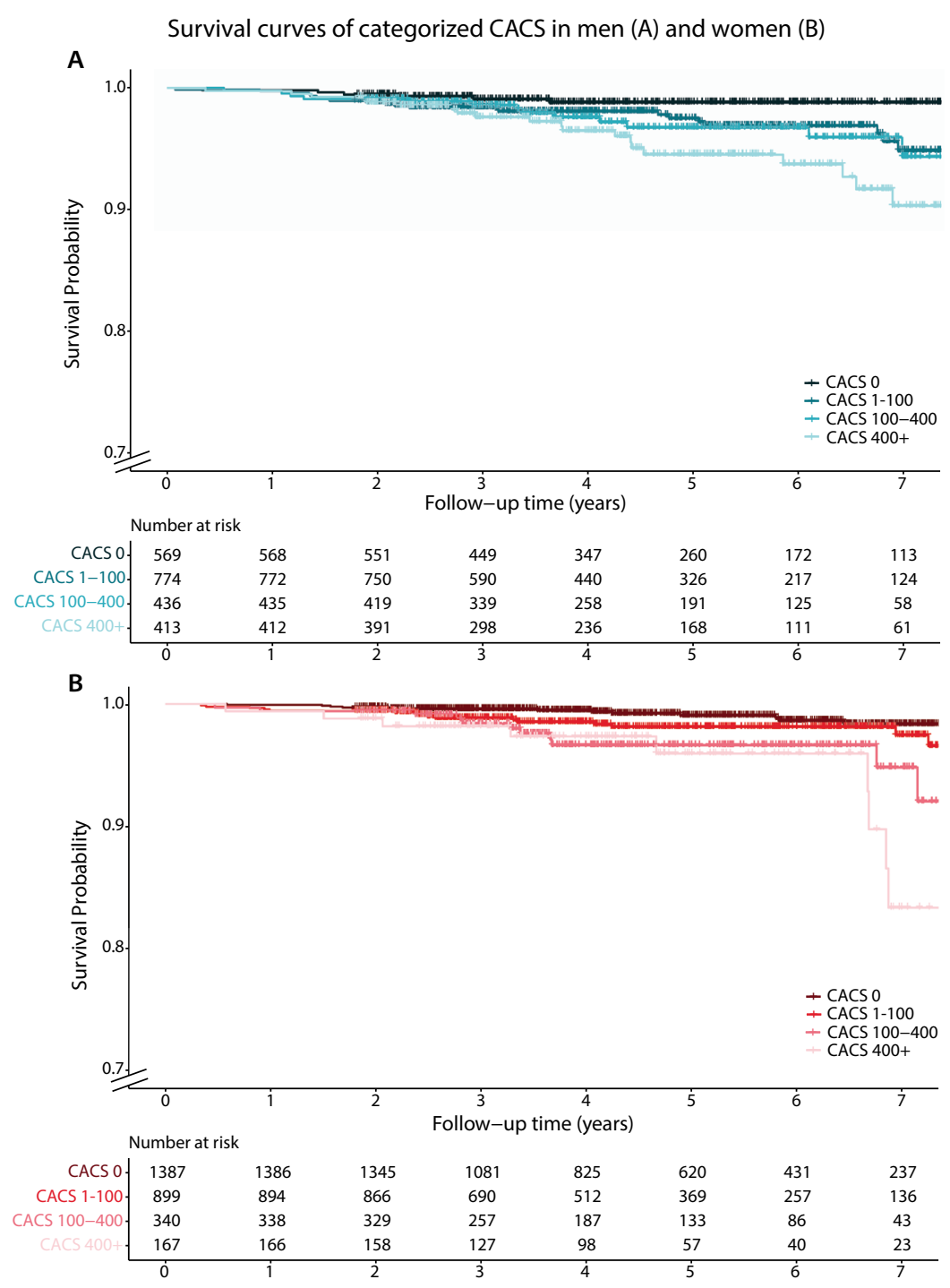

Figure 2. Survival of women and men by absolute CACS

(3.5\%) men and 22 (1.5\%) women died. Compared to the population in which only CACS was available, these women and men did not differ in baseline characteristics (for direct comparison and baseline characteristics per CACS category see Supplementary Table 3 and 4, respectively). In the population in whom both CACS and information on stenosis degree was available, the relation between calcification measures and mortality was comparable to the relation found in the total population 
(Table 4). In men, the discriminative power of the model did not improve when degree of stenosis was added (C-statistic changed from $0.63,95 \% \mathrm{Cl}: 0.55-0.71$ based on CACS to $0.59,95 \% \mathrm{Cl}: 0.51-0.67$ after addition of stenosis to the model). In women, the performance to predict mortality improved slightly (C-statistic: $0.68,95 \% \mathrm{Cl}$ : $0.58-0.78$, and $0.72,95 \% \mathrm{Cl}: 0.61-0.83$, respectively). However, this improvement was not significant and thus no hard conclusions can be drawn.

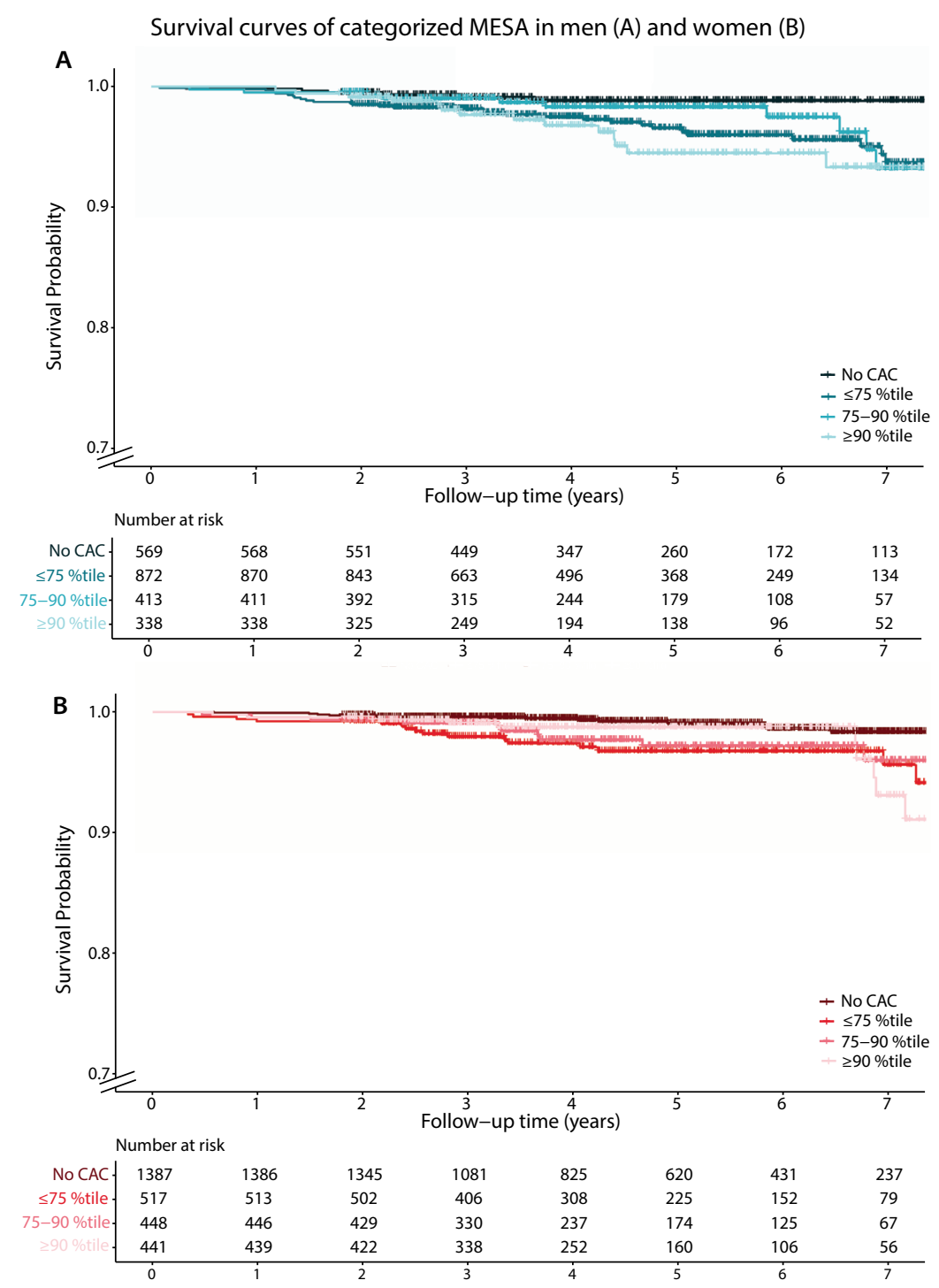

Figure 3. Survival of women and men by sex- and race/ethnicity specific percentiles (MESA) 
Table 2. Mortality prediction as a function of absolute and age-, sex- and race/ethnicity specific percentiles of CACS in women (above) and men (below)

\begin{tabular}{|c|c|c|c|c|c|}
\hline Women & Model & $\mathbf{n}$ & events & HR $(95 \% \mathrm{Cl})$ & $\begin{array}{l}\text { C-statistic } \\
(95 \% \mathrm{Cl})\end{array}$ \\
\hline \multicolumn{6}{|c|}{ Absolute CACS } \\
\hline & Continuous & 2793 & 53 & $1.0(1.0-1.0)$ & $0.66(0.58-0.74)$ \\
\hline & Categorized & & & & $0.65(0.57-0.73)$ \\
\hline & CACS 0 & 1387 & 14 & reference & \\
\hline & CACS $1-100$ & 899 & 18 & $2.1(1.0-2.1)$ & \\
\hline & CACS $101-400$ & 340 & 12 & $4.0(1.8-8.6)$ & \\
\hline & CACS $>400$ & 167 & $<10$ & $5.7(2.5-13.2)$ & \\
\hline \multicolumn{6}{|c|}{ Sex-, age-, and race/ethnicity-specific percentiles } \\
\hline & $\begin{array}{l}\text { Continuous } \\
\text { adjusted for any } \\
\text { calcification }\end{array}$ & 2793 & 53 & & $0.65(0.57-0.73)$ \\
\hline & MESA percentile & & & $0.4(0.1-1.5)$ & \\
\hline & Any calcification & & & $6.1(1.9-19.1)$ & \\
\hline & Categorized & & & & $0.64(0.57-0.72)$ \\
\hline & No calcification & 1387 & 14 & reference & \\
\hline & $<75^{\text {th }}$ percentile & 517 & 18 & $3.5(1.8-7.1)$ & \\
\hline & $\begin{array}{l}75^{\text {th }}-90^{\text {th }} \\
\text { percentile }\end{array}$ & 448 & 11 & $2.7(1.2-5.9)$ & \\
\hline & $>90^{\text {th }}$ percentile & 441 & 10 & $2.5(1.1-5.6)$ & \\
\hline Men & Model & n & events & $\mathrm{HR}(95 \% \mathrm{Cl})$ & $\begin{array}{l}\text { C-statistic } \\
\text { (95\%Cl) }\end{array}$ \\
\hline \multicolumn{6}{|c|}{ Absolute CACS } \\
\hline & Continuous & 2192 & 63 & $1.0(1.0-1.0)$ & $0.62(0.55-0.69)$ \\
\hline & Categorized & & & & $0.60(0.53-0.67)$ \\
\hline & CACS 0 & 569 & $<10$ & reference & \\
\hline & CACS $1-100$ & 774 & 23 & $3.0(1.2-7.5)$ & \\
\hline & CACS $101-400$ & 436 & 14 & $3.3(1.3-8.7)$ & \\
\hline & CACS $>400$ & 413 & 20 & $5.1(2.0-12.7)$ & \\
\hline \multicolumn{6}{|c|}{ Sex-, age-, and race/ethnicity-specific percentiles } \\
\hline & $\begin{array}{l}\text { Continuous } \\
\text { adjusted for any } \\
\text { calcification }\end{array}$ & 2192 & 63 & & $0.59(0.51-0.67)$ \\
\hline & Percentile & & & $0.5(0.2-1.5)$ & \\
\hline & Any calcification & & & $5.7(1.9-16.8)$ & \\
\hline & Categorized & & & & \\
\hline & No calcification & 569 & $<10$ & reference & $0.61(0.54-0.67)$ \\
\hline & $<75^{\text {th }}$ percentile & 872 & 33 & $3.9(1.6-9.3)$ & \\
\hline & $\begin{array}{l}75^{\text {th }}-90^{\text {th }} \\
\text { percentile }\end{array}$ & 413 & 10 & $2.5(0.9-7.0)$ & \\
\hline & $>90^{\text {th }}$ percentile & 338 & 14 & $4.3(1.6-11.1)$ & \\
\hline
\end{tabular}


Table 3. Baseline characteristics of women and men that underwent cardiac CT angiography

\begin{tabular}{|c|c|c|c|}
\hline & Overall & Women & Men \\
\hline$n$ & 2715 & 1385 & 1330 \\
\hline Age in years (mean (SD)) & $60(8)$ & $60(8)$ & $59(8)$ \\
\hline Body mass index (mean (SD)) & $27(4)$ & $27(5)$ & $27(4)$ \\
\hline \multicolumn{4}{|l|}{ Complaints, n (\%) } \\
\hline Chest pain or discomfort & $1499(55)$ & $801(58)$ & $698(53)$ \\
\hline Dyspnea & $295(11)$ & $167(12)$ & $128(10)$ \\
\hline Fatigue & $93(3)$ & $47(3)$ & $46(4)$ \\
\hline Palpitations & $272(10)$ & $163(12)$ & $109(8)$ \\
\hline Collapse & $18(1)$ & $<10$ & $>10$ \\
\hline \multicolumn{4}{|l|}{ Smoking status, n (\%) } \\
\hline Current & $809(32)$ & $428(34)$ & $381(31)$ \\
\hline Former & $936(37)$ & $461(36)$ & $475(38)$ \\
\hline Never & $765(31)$ & $380(30)$ & $385(31)$ \\
\hline Diabetes Mellitus, n (\%) & $211(8)$ & $97(7)$ & $114(9)$ \\
\hline Hypertension, n (\%) & $885(33)$ & $518(38)$ & $367(28)$ \\
\hline Dyslipidemia, n (\%) & $497(18)$ & 263 (19) & $234(18)$ \\
\hline \multicolumn{4}{|l|}{ Degree of Stenosis, $n(\%)$} \\
\hline $0 \%$ & 792 (29) & $527(38)$ & $265(20)$ \\
\hline $1-49 \%$ & $1114(41)$ & $563(41)$ & $551(41)$ \\
\hline $50-70 \%$ & $431(16)$ & $168(12)$ & $263(20)$ \\
\hline$>70 \%$ & $378(14)$ & $127(9)$ & $251(19)$ \\
\hline CACS (median [IQR]) & $34[0,162]$ & $14[0,92]$ & $67[6,267]$ \\
\hline \multicolumn{4}{|l|}{ CACS category, $\mathrm{n}(\%)$} \\
\hline 0 & $682(25)$ & $472(34)$ & $210(16)$ \\
\hline $1-100$ & $1150(42)$ & $585(42)$ & $565(43)$ \\
\hline $101-400$ & $598(22)$ & $251(18)$ & $347(26)$ \\
\hline$>400$ & $285(11)$ & $77(6)$ & $208(16)$ \\
\hline \multicolumn{4}{|l|}{ Examinations during follow-up: } \\
\hline At least one CAG, $\mathrm{n}(\%)$ & $553(20)$ & 205 (15) & $348(26)$ \\
\hline At least one $\mathrm{PCl}$ or $\mathrm{CABG}, \mathrm{n}(\%)$ & $234(9)$ & $66(5)$ & $168(13)$ \\
\hline Deaths, n (\%) & $68(3)$ & $22(2)$ & $46(4)$ \\
\hline Cardiovascular death, $\mathrm{n}(\%)$ & $10(0.4)$ & & \\
\hline Years of follow up (median [IQR]) & $4.6[3.2,6.3]$ & $4.6[3.2,6.3]$ & $4.7[3.2,6.4]$ \\
\hline
\end{tabular}

CACS, coronary artery calcium score; CAG, coronary angiography; $\mathrm{PCl}$, percutaneous coronary intervention; CABG, coronary artery bypass graft 
Table 4. Mortality prediction as a function of absolute CACS and degree of stenosis in women and men

\begin{tabular}{|c|c|c|c|c|c|}
\hline Women & Model & n & events & HR $(95 \% \mathrm{Cl})$ & C-statistic (95\%Cl) \\
\hline \multicolumn{6}{|c|}{ Absolute CACS } \\
\hline \multirow{6}{*}{+ stenosis } & Continuous & 1385 & 22 & $1.0(1.0-1.0)$ & $0.68(0.58-0.78)$ \\
\hline & Degree of stenosis & & & & $0.72(0.61-0.83)$ \\
\hline & $0 \%$ & 527 & $<10$ & reference & \\
\hline & $1-49 \%$ & 563 & 14 & $6.9(1.6-30.6)$ & \\
\hline & $50-70 \%$ & 168 & $<10$ & $4.9(0.9-28.6)$ & \\
\hline & $>70 \%$ & 127 & $<10$ & $2.8(0.3-22.4)$ & \\
\hline Men & Model & n & events & HR $(95 \% \mathrm{Cl})$ & C-statistic $(95 \% \mathrm{Cl})$ \\
\hline \multicolumn{6}{|c|}{ Absolute CACS } \\
\hline \multirow{6}{*}{+ stenosis } & Continuous & 1330 & 46 & $1.0(1.0-1.0)$ & $0.63(0.55-0.71)$ \\
\hline & Degree of stenosis & & & & $0.59(0.51-0.67)$ \\
\hline & $0 \%$ & 265 & $<10$ & reference & \\
\hline & $1-49 \%$ & 551 & 22 & $4.5(1.3-15.3)$ & \\
\hline & $50-70 \%$ & 263 & 10 & $3.9(1.1-14.7)$ & \\
\hline & $>70 \%$ & 251 & 11 & $4.2(1.1-16.3)$ & \\
\hline
\end{tabular}

\section{DISCUSSION}

Our data showed that both absolute and sex-, age-, and race/ethnicity-specific percentiles of CACS perform equally well in predicting mortality in symptomatic women and men who visit outpatient cardiology clinics in a real-world setting. Hence, for discrimination of mortality in symptomatic women and men there is no need for sex-, age-, and race/ethnicity-specific percentiles to quantify the amount of coronary artery calcification. CACS predicts mortality with moderate performance, comparable to the performance in asymptomatic women and men (12). Finally, the data hint that in women, the discriminative power of CACS for mortality might be higher when degree of stenosis was included in the model. However, these results should be interpreted with caution as the improvement was minimal and not significant. In men, addition of degree of stenosis did not result in better prediction of mortality. This subtle sex-difference might be due to the presence of non-calcified plaques causing symptoms in women (6). This type of plaques remains (partly) unappreciated when using CACS only for mortality prediction. 
To the best of our knowledge, this is the first sex-stratified study that evaluates the value of CACS and sex-, age-, and race/ethnicity-specific percentiles in a large, contemporary clinical care cohort of symptomatic individuals. In the scientific literature only limited data on the prognostic value of CACS in symptomatic patients is available. The only recent study that focused on symptomatic women ( $n=1972$, $51 \%$ ) and men ( $n=1868,49 \%$ ) suspected of CAD was the PROMISE trial (3). In the PROMISE trial the added value of degree of stenosis, and even specific (high risk) plaque characteristics, was incorporated in prognostic models. They showed a high prognostic value of CACS, which further increased after addition of stenosis degree. However, their analysis was not performed stratified by sex, hampering any comparison to our data and impeding the analysis of sex differences, even though plaque composition is significantly different between the sexes. They also showed that the recently developed Coronary Artery Disease Reporting and Data System (CAD-RADS) (17) classification provides the highest prognostic value for cardiovascular events.

Our results in symptomatic women and men are in line with the results of the MESA study, which was conducted in non-symptomatic general population individuals. The MESA study demonstrated that absolute CACS outperforms sex-, age-, and race/ ethnicity-specific percentiles for event prediction (12). We also found that the discriminative capacity remains intact when categorizing CACS, comparable to the results of the MESA study. This finding is valuable for clinical use, as hazard ratios derived from categories are easier to interpret than hazard ratios derived from continuous values. Furthermore, other studies in asymptomatic individuals concluded that CACS equally predicted mortality risk between the sexes $(1,18)$, despite the finding that women had a lower CACS compared to men (1) and while significant sex differences were present in the cumulative mortality (18). Most studies reported that CACS may be a better predictor for mortality in women compared to men $(14,15$, 18). This suggestion is in line with the slightly higher C-statistic for the CACS-model we have described in women. In addition, Wang et al. noted that sex-specific CACS percentiles tended to better stratify risk in women than men as opposed to absolute scores (14). The reason for the difference might be the inclusion of an asymptomatic and older population in the study by Wang et al.

The strength of the study presented here lies in the real-world population in whom cardiac CT is often used as a primary diagnostic, yet the power of cardiac CT is not often studied. This population is best described as a symptomatic population, referred to a specialized cardiac screening center, which is positioned in the care trajectory between general practitioners and hospital care. Furthermore, the link to the database 
of Statistics Netherland provided high-quality long-term follow-up data on mortality and information on country of birth.

Use of EHR data also has inherent limitations. First, data were not primarily collected for study purposes. Therefore, part of the population that underwent cardiac CT could not be included, due to insufficient documentation of CT results in the EHR. Second, as CT angiography was only performed based on referral by the cardiologist and/or local scan protocol, not all patients underwent CT angiography. Thus, the subanalysis focusing on degree of stenosis, based on CT angiography results, could only be performed in a subset of patients. This significantly reduced the power of our statistical analysis and might have led to selection bias. To evaluate this bias, we have compared the overall and subpopulation and repeated the analyses in the subpopulation. The populations were comparable on known baseline characteristics. Moreover, the results of the use of CACS and sex-, age- and race/ethnicity-specific percentiles for risk stratification did not significantly differ. This suggests that increased discriminative power of CACS when degree of stenosis was added to the model is generalizable. Third, as stenosis severity was retrieved from textual reports in which degree of stenosis was not always quantified, the interpretation of the grade of stenosis could also have led to uncertainty in our data. Fourth, we did not take treatment differences into account between different groups. These differences may explain the decreasing hazard ratio with an increasing degree of coronary stenosis, although this decrease was not found to be significant. Finally, as we have used country of origin as a surrogate for race/ethnicity, as the latter was unavailable in the database of Statistics Netherlands, the categories used in the original MESA calculations and our study population were not identical. To assess whether this has influenced our results we have performed sensitivity analysis in which we only studied women and men that originated from Europe, assuming they all have the Caucasian race. These results were not significantly different from the main analyses. Moreover, as our population was mainly coming from Europe, our results might not be applicable to women and men of other ethnic populations.

We found that adding degree of stenosis to the survival model might slightly improve the discriminative power in women, but not in men. However, this improvement in women was not statistically significant and the confidence intervals largely overlapped. Even though we have shown a moderate to good prognostic value of CACS in both symptomatic women and men, our results warrant evaluation beyond CACS alone for optimal mortality risk prediction as the C-statistics were below 0.70 . This might be especially relevant in women, in whom information on the degree of stenosis possibly has additional value due to presence of non-calcified plaques. In this study 
we were unable to assess the added value of degree of stenosis properly due limited and incomplete data. Nevertheless, the small difference between women and men we found after degree of stenosis was added to the model stresses the importance of a sex-specific view on individuals suspect of or suffering from CAD. Replication of this study in larger trials or populations is going to contribute to confirm the hypotheses proposed in this study, regarding the importance of non-calcified plaques in women for cardiovascular morbidity and mortality.

\section{CONCLUSION}

In symptomatic women and men at outpatient cardiology clinics absolute CACS predicts mortality with a moderately good performance. Sex-, age-, and race/ethnicityspecific percentiles did not perform better compared to absolute CACS, thus there is no need to use them. Including degree of stenosis in the model might slightly improve mortality risk prediction in women, but not in men. 


\section{REFERENCES}

1. Nakanishi R, Li D, Blaha MJ, Whelton SP, Darabian S, Flores FR, et al. All-cause mortality by age and gender based on coronary artery calcium scores. European Heart Journal-Cardiovascular Imaging. 2016;17(11):1305-14.

2. Mittal TK, Pottle A, Nicol E, Barbir M, Ariff B, Mirsadraee S, et al. Prevalence of obstructive coronary artery disease and prognosis in patients with stable symptoms and a zero-coronary calcium score. European Heart JournalCardiovascular Imaging. 2017;18(8):922-9.

3. Bittner DO, Mayrhofer T, Budoff M, Szilveszter B, Foldyna B, Hallett TR, et al. Prognostic value of coronary CTA in stable chest pain: CAD-RADS, CAC, and cardiovascular events in PROMISE. Cardiovascular Imaging. 2020;13(7):1534-45.

4. Knuuti J, Wijns W, Saraste A, Capodanno D, Barbato E, Funck-Brentano C, et al. 2019 ESC Guidelines for the diagnosis and management of chronic coronary syndromes: the Task Force for the diagnosis and management of chronic coronary syndromes of the European Society of Cardiology (ESC). European heart journal. 2020;41(3):407-77.

5. Agatston AS, Janowitz WR, Hildner FJ, Zusmer NR, Viamonte M, Detrano R. Quantification of coronary artery calcium using ultrafast computed tomography. Journal of the American College of Cardiology. 1990;15(4):827-32.

6. Plank F, Beyer C, Friedrich G, Wildauer M, Feuchtner G. Sex differences in coronary artery plaque composition detected by coronary computed tomography: quantitative and qualitative analysis. Netherlands Heart Journal. 2019;27(5):272-80.

7. Williams MC, Kwiecinski J, Doris M, McElhinney P, D'Souza MS, Cadet S, et al. Sex-Specific Computed Tomography Coronary Plaque Characterization and Risk of Myocardial Infarction. JACC: Cardiovascular Imaging. 2021.

8. Hussain A, Ballantyne CM, Nambi V. Zero Coronary Artery Calcium Score: Desirable, but Enough? Circulation. 2020;142(10):917-9.

9. Bild DE, Detrano R, Peterson D, Guerci A, Liu K, Shahar E, et al. Ethnic differences in coronary calcification: the Multi-Ethnic Study of Atherosclerosis (MESA). Circulation. 2005;111(10):1313-20.

10. Hoff JA, Chomka EV, Krainik AJ, Daviglus M, Rich S, Kondos GT. Age and gender distributions of coronary artery calcium detected by electron beam tomography in 35,246 adults. The American journal of cardiology. 2001;87(12):1335-9.

11. Budoff MJ, Nasir K, McClelland RL, Detrano R, Wong N, Blumenthal RS, et al. Coronary calcium predicts events better with absolute calcium scores than age-gender-race percentiles-The Multi-Ethnic Study of Atherosclerosis (MESA). Journal of the American College of Cardiology. 2009;53(4):345.

12. RI M. Chung H, Detrano R, post W, Kronmal R a. Distribution of coronary artery calcium by race, gender, and age: results from the multiethnic study of atherosclerosis (MESA). Circulation. 2006;113:30-7.

13. Shaw LJ, Min JK, Nasir K, Xie JX, Berman DS, Miedema MD, et al. Sex differences in calcified plaque and long-term cardiovascular mortality: observations from the CAC Consortium. European heart journal. 2018;39(41):3727-35.

14. Wang FM, Rozanski A, Arnson Y, Budoff MJ, Miedema MD, Nasir K, et al. Cardiovascular and all-cause mortality risk by coronary artery calcium scores and percentiles among older adult males and females. The American Journal of Medicine. 2021;134(3):341-50. e1.

15. Kelkar AA, Schultz WM, Khosa F, Schulman-Marcus J, O'Hartaigh BW, Gransar H, et al. Long-term prognosis after coronary artery calcium scoring among low-intermediate risk women and men. Circulation: Cardiovascular Imaging. 2016;9(4):e003742.

16. Bots SH, Siegersma KR, Onland-Moret NC, Asselbergs FW, Somsen GA, Tulevski II, et al. Routine clinical care data from thirteen cardiac outpatient clinics: design of the Cardiology Centers of the Netherlands (CCN) database. BMC cardiovascular disorders. 2021;21(1):1-9.

17. Foldyna B, Szilveszter B, Scholtz J-E, Banerji D, Maurovich-Horvat P, Hoffmann U. CAD-RADS-a new clinical decision support tool for coronary computed tomography angiography. European radiology. 2018;28(4):1365-72.

18. Raggi P, Shaw LJ, Berman DS, Callister TQ. Gender-based differences in the prognostic value of coronary calcification. Journal of Women's Health. 2004;13(3):273-83. 


\section{SUPPLEMENTARY MATERIAL}

Supplementary Table 1. Baseline characteristics of study population per sex stratified per CACS category

\begin{tabular}{|c|c|c|c|c|}
\hline Women & CACS $=0$ & CACS $1-100$ & CACS $101-400$ & CACS $>400$ \\
\hline $\mathrm{n}$ & 1387 & 899 & 340 & 167 \\
\hline Age in years (mean (SD)) & $56(7)$ & $62(8)$ & $64(8)$ & $67(8)$ \\
\hline Body mass index (mean (SD)) & $26(5)$ & $27(5)$ & $27(5)$ & $27(5)$ \\
\hline \multicolumn{5}{|l|}{ Smoking status, $\mathrm{n}(\%)$} \\
\hline Current & $441(34)$ & $270(32)$ & $99(32)$ & $49(31)$ \\
\hline Former & $383(30)$ & $301(36)$ & $129(42)$ & $62(40)$ \\
\hline Never & $465(36)$ & $262(32)$ & $82(27)$ & $45(29)$ \\
\hline Diabetes Mellitus, n (\%) & $78(6)$ & $75(8)$ & $30(9)$ & $30(18)$ \\
\hline Hypertension, $\mathrm{n}(\%)$ & $349(25)$ & 352 (39) & $164(48)$ & $89(53)$ \\
\hline Dyslipidemia, n (\%) & $167(12)$ & $179(20)$ & $82(24)$ & $49(29)$ \\
\hline \multicolumn{5}{|l|}{ Degree of stenosis, $n(\%)$} \\
\hline $0 \%$ & $432(92)$ & $82(14)$ & $11(4)$ & $<10$ \\
\hline $1-49 \%$ & $34(7)$ & $399(68)$ & $110(44)$ & $15-20$ \\
\hline $50-70 \%$ & $<10$ & $65(11)$ & $74(30)$ & $23(30)$ \\
\hline$>70 \%$ & $<10$ & $39(7)$ & $56(22)$ & $32(42)$ \\
\hline \multicolumn{5}{|l|}{ Examinations during follow-up: } \\
\hline At least one CAG, $\mathrm{n}(\%)$ & 10 tot 15 & $90(10)$ & $114(34)$ & $90(54)$ \\
\hline At least one $\mathrm{PCl}$ or $\mathrm{CABG}, \mathrm{n}(\%)$ & $<10$ & $24(3)$ & $23(7)$ & $41(25)$ \\
\hline Mortality rate, $\mathrm{n}(\%)$ & $14(1)$ & $18(2)$ & $12(4)$ & $<10$ \\
\hline Years of follow up (median [IQR]) & $5[3,6]$ & $4[3,6]$ & $4[3,6]$ & $4[3,6]$ \\
\hline Men & CACS $=0$ & CACS $1-100$ & CACS $101-400$ & CACS $>400$ \\
\hline $\mathrm{n}$ & 569 & 774 & 436 & 413 \\
\hline Age in years (mean (SD)) & $54(7)$ & $58(8)$ & $61(8)$ & $64(7)$ \\
\hline Body mass index (mean (SD)) & $27(4)$ & $27(4)$ & $27(4)$ & $27(4)$ \\
\hline \multicolumn{5}{|l|}{ Smoking status, $\mathrm{n}(\%)$} \\
\hline Current & $165(32)$ & $217(30)$ & $113(28)$ & $122(32)$ \\
\hline Former & $168(32)$ & $259(36)$ & $174(42)$ & $174(45)$ \\
\hline Never & $191(37)$ & $245(34)$ & $123(30)$ & $91(24)$ \\
\hline Diabetes Mellitus, n (\%) & $23(4)$ & $64(8)$ & $35(8)$ & $61(15)$ \\
\hline Hypertension, n (\%) & $113(20)$ & $197(26)$ & $153(35)$ & $164(40)$ \\
\hline Dyslipidemia, n (\%) & $57(10)$ & $129(17)$ & $85(20)$ & $102(25)$ \\
\hline \multicolumn{5}{|l|}{ Degree of stenosis, $n(\%)$} \\
\hline $0 \%$ & $181(86.2)$ & $68(12)$ & $14(4)$ & $<10$ \\
\hline $1-49 \%$ & $25(12)$ & $355(63)$ & $128(37)$ & $40-45$ \\
\hline $50-70 \%$ & $<10$ & $94(17)$ & $109(31)$ & $57(27)$ \\
\hline$>70 \%$ & $<10$ & $48(9)$ & $96(28)$ & $106(51)$ \\
\hline \multicolumn{5}{|l|}{ Examinations during follow-up: } \\
\hline At least one CAG, $\mathrm{n}(\%)$ & $<10$ & $109(14)$ & $147(34)$ & $239(58)$ \\
\hline At least one $\mathrm{PCl}$ or $\mathrm{CABG}, \mathrm{n}(\%)$ & $<10$ & $38(5)$ & $67(15)$ & $111(27)$ \\
\hline Mortality rate, $\mathrm{n}(\%)$ & $<10$ & $23(3)$ & $14(3)$ & $20(5)$ \\
\hline Years of follow up (median [IQR]) & $5[3,6]$ & $4[3,6]$ & $4[3,6]$ & $4[3,6]$ \\
\hline
\end{tabular}

CACS, coronary artery calcium score; CAG, coronary angiography; $\mathrm{PCl}$, percutaneous coronary intervention; CABG, coronary artery bypass graft 
Supplementary Table 2. Mortality prediction as a function of absolute and age-, sex- and ethnicity specific percentiles of CACS in women (above) and men (below), only performed in European women and men

\begin{tabular}{|c|c|c|c|c|c|}
\hline European women & Model & n & events & $\mathrm{HR}(95 \% \mathrm{Cl})$ & $\begin{array}{r}\text { C-statistic } \\
(95 \% \mathrm{Cl})\end{array}$ \\
\hline \multicolumn{6}{|l|}{ Absolute CACS } \\
\hline & Continuous & 2411 & 47 & $1.0(1.0-1.0)$ & $0.67(0.59-0.76)$ \\
\hline & Categorized & & & & $0.67(0.59-0.75)$ \\
\hline & CACS 0 & 1185 & 11 & reference & \\
\hline & CACS $1-100$ & 773 & 16 & & \\
\hline & CACS $101-400$ & 304 & 12 & & \\
\hline & CACS $>400$ & 149 & $<10$ & & \\
\hline \multicolumn{6}{|c|}{ Sex-, age-, and race/ethnicity-specific percentiles } \\
\hline & Continuous adjusted for any calcification & 2411 & 47 & & $0.61(0.58-0.75)$ \\
\hline & MESA percentile & & & $0.4(0.1-1.7)$ & \\
\hline & Any calcification & & & $6.9(2.0-23.7)$ & \\
\hline & Categorized & & & & $0.66(0.58-0.74)$ \\
\hline & No calcification & 1185 & 11 & reference & \\
\hline & $<75^{\text {th }}$ percentile & 466 & 16 & $3.8(1.8-8.3)$ & \\
\hline & $75^{\text {th }}-90^{\text {th }}$ percentile & 386 & 11 & $3.4(1.5-8.0)$ & \\
\hline & $>90^{\text {th }}$ percentile & 374 & $<10$ & $3.0(1.2-7.1)$ & \\
\hline European men & Model & n & events & $\mathrm{HR}(95 \% \mathrm{Cl})$ & C-statistic \\
\hline \multicolumn{6}{|l|}{ Absolute CACS } \\
\hline & Continuous & 1898 & 57 & $1.0(1.0-1.0)$ & $0.64(0.56-0.72$ \\
\hline & Categorized & & & & $0.62(0.55-0.70)$ \\
\hline & CACS 0 & 480 & $<10$ & reference & \\
\hline & CACS $1-100$ & 665 & 20 & $3.9(1.3-11.3)$ & \\
\hline & CACS $101-400$ & 380 & 13 & $4.4(1.4-13.6)$ & \\
\hline & CACS $>400$ & 373 & 20 & $7.1(2.4-20.7)$ & \\
\hline \multicolumn{6}{|c|}{ Sex-, age-, and race/ethnicity-specific percentiles } \\
\hline & Continuous adjusted for any calcification & 1898 & 57 & & $0.59(0.51-0.67)$ \\
\hline & MESA percentile & & & $0.5(0.2-1.7)$ & \\
\hline & Any calcification & & & $7.3(2.1-25.6)$ & \\
\hline & Categorized & & & & $0.62(0.55-0.69)$ \\
\hline & No calcification & 480 & $<10$ & reference & \\
\hline & $<75^{\text {th }}$ percentile & 763 & 30 & $5.1(1.8-14.5)$ & \\
\hline & $75^{\text {th }}-90^{\text {th }}$ percentile & 360 & $<10$ & $3.3(1.0-10.6)$ & \\
\hline & $>90^{\text {th }}$ percentile & 295 & 14 & $6.1(2.0-18.6)$ & \\
\hline
\end{tabular}


Supplementary Table 3. Direct comparison of CT and CTA population

\begin{tabular}{|c|c|c|}
\hline & CT population & Subgroup with CTA \\
\hline $\mathrm{n}$ & 4985 & 2715 \\
\hline Female, n (\%) & $2793(56)$ & $1385(51)$ \\
\hline Age in years (mean (SD)) & $59(8)$ & $57(8)$ \\
\hline Body mass index (mean (SD)) & $27(4)$ & $27(4)$ \\
\hline \multicolumn{3}{|l|}{ Complaints, n (\%) } \\
\hline Chest pain or discomfort & $2683(54)$ & $1499(55)$ \\
\hline Dyspnea & $538(11)$ & $295(11)$ \\
\hline Fatigue & $172(4)$ & $93(3)$ \\
\hline Palpitations & $463(9)$ & $272(10)$ \\
\hline Collapse & $27(0.5)$ & $18(0.7)$ \\
\hline \multicolumn{3}{|l|}{ Smoking status, $n$ (\%) } \\
\hline Current & $1476(32)$ & $809(32)$ \\
\hline Former & $1650(36)$ & $936(37)$ \\
\hline Never & $1504(33)$ & $765(31)$ \\
\hline Diabetes Mellitus, n (\%) & $396(8)$ & $211(8)$ \\
\hline Hypertension, n (\%) & $1581(32)$ & $885(33)$ \\
\hline Dyslipidemia, n (\%) & $850(17)$ & $497(18)$ \\
\hline CACS (median [IQR]) & $8[0,121]$ & $35[0,162]$ \\
\hline \multicolumn{3}{|l|}{ CACS category, $\mathrm{n}(\%)$} \\
\hline 0 & 1956 (39) & $682(25)$ \\
\hline $1-100$ & $1673(34)$ & $1150(42)$ \\
\hline $101-400$ & $776(16)$ & $598(22)$ \\
\hline$>400$ & $580(12)$ & $285(11)$ \\
\hline Deaths, n (\%) & $116(2)$ & $68(3)$ \\
\hline Cardiovascular death, n (\%) & $22(0.4)$ & $10(0.4)$ \\
\hline
\end{tabular}


Supplementary Table 4. Baseline characteristics of CTA study population per sex stratified per CACS category

\begin{tabular}{|c|c|c|c|c|}
\hline Women & CACS $=0$ & CACS $1-100$ & CACS $101-400$ & CACS $>400$ \\
\hline $\mathrm{n}$ & 472 & 585 & 251 & 77 \\
\hline Age in years (mean (SD)) & $56(7)$ & $61(8)$ & $64(8)$ & $66(8)$ \\
\hline Body mass index (mean (SD)) & $26(5)$ & $27(5)$ & $27(5)$ & $27(4)$ \\
\hline \multicolumn{5}{|l|}{ Smoking status, $\mathrm{n}(\%)$} \\
\hline Current & $168(39)$ & $173(32)$ & $68(29)$ & $19(27)$ \\
\hline Former & $136(32)$ & $200(37)$ & $99(43)$ & $26(37)$ \\
\hline Never & $123(29)$ & $166(31)$ & $66(28)$ & $25(36)$ \\
\hline Diabetes Mellitus, n (\%) & $22(5)$ & $43(7)$ & $21(8)$ & $10-15(13-19)$ \\
\hline Hypertension, n (\%) & $135(29)$ & $221(38)$ & $122(49)$ & $40(52)$ \\
\hline Dyslipidemia, n (\%) & $64(14)$ & $110(19)$ & $66(26)$ & $23(30)$ \\
\hline \multicolumn{5}{|l|}{ Degree of stenosis, $n(\%)$} \\
\hline $0 \%$ & $432(92)$ & $82(14)$ & $11(4)$ & $<10(<13)$ \\
\hline $1-49 \%$ & $34(7)$ & $399(68)$ & $110(44)$ & $20-25(25-32)$ \\
\hline $50-70 \%$ & $<10(<2)$ & $65(11)$ & $74(30)$ & $23(30)$ \\
\hline$>70 \%$ & $<10(<2)$ & $39(7)$ & $56(22)$ & $32(42)$ \\
\hline \multicolumn{5}{|l|}{ Examinations during follow-up: } \\
\hline At least one CAG, $\mathrm{n}(\%)$ & $<10(<2)$ & $72(12)$ & $96(38)$ & $32(42)$ \\
\hline At least one $\mathrm{PCl}$ or $\mathrm{CABG}, \mathrm{n}(\%)$ & $<10(<2)$ & $23(4)$ & $22(9)$ & $20(26)$ \\
\hline Mortality rate, $\mathrm{n}(\%)$ & $<10(<2)$ & $<10(<2)$ & $<10(<4)$ & $<10(<13)$ \\
\hline Years of follow up (median [IQR]) & $5[3,7]$ & $4[3,6]$ & $4[3,6]$ & $4[3,5]$ \\
\hline Men & CACS $=0$ & CACS $1-100$ & CACS $101-400$ & CACS $>400$ \\
\hline $\mathrm{n}$ & 210 & 565 & 347 & 208 \\
\hline Age in years (mean (SD)) & $54(6)$ & $58(8)$ & $61(8)$ & $63(8)$ \\
\hline Body mass index (mean (SD)) & $26(4)$ & $27(4)$ & $27(4)$ & $27(3)$ \\
\hline \multicolumn{5}{|l|}{ Smoking status, $\mathrm{n}(\%)$} \\
\hline Current & $75(40)$ & $160(30)$ & $85(26)$ & $61(32)$ \\
\hline Former & $55(29)$ & $193(36)$ & $146(45)$ & $81(42)$ \\
\hline Never & $57(31)$ & $181(34)$ & $96(29)$ & $51(26)$ \\
\hline Diabetes Mellitus, n (\%) & $<10$ & $48(9)$ & $28(8)$ & $29(14)$ \\
\hline Hypertension, n (\%) & $34(16)$ & $143(25)$ & $118(34)$ & $72(35)$ \\
\hline Dyslipidemia, n (\%) & $23(11)$ & $94(17)$ & $65(19)$ & $52(25)$ \\
\hline \multicolumn{5}{|l|}{ Degree of stenosis, n (\%) } \\
\hline $0 \%$ & $181(86)$ & $68(12)$ & $14(4)$ & $<10(<4)$ \\
\hline $1-49 \%$ & $25(12)$ & $355(63)$ & $128(37)$ & $40-45(19-22)$ \\
\hline $50-70 \%$ & $<10(<4)$ & $94(17)$ & $109(31)$ & $57(27)$ \\
\hline$>70 \%$ & $<10(<4)$ & $48(9)$ & $96(28)$ & $106(51)$ \\
\hline \multicolumn{5}{|l|}{ Examinations during follow-up: } \\
\hline At least one CAG, $\mathrm{n}(\%)$ & $<10(<4)$ & $92(16)$ & $127(37)$ & $123(59)$ \\
\hline At least one $\mathrm{PCl}$ or $\mathrm{CABG}, \mathrm{n}(\%)$ & $<10(<4)$ & $33(6)$ & $61(18)$ & $73(35)$ \\
\hline Deaths, n (\%) & $<10(<4)$ & $21(4)$ & $12(4)$ & $12(6)$ \\
\hline Years of follow up (median [IQR]) & $5[4,7]$ & $4[3,6]$ & $5[3,6]$ & $4[3,6]$ \\
\hline
\end{tabular}

CACS, coronary artery calcium score; CAC, coronary artery calcium; $C A G$, coronary angiography; $\mathrm{PCl}$, percutaneous coronary intervention; CABG, coronary artery bypass graft 

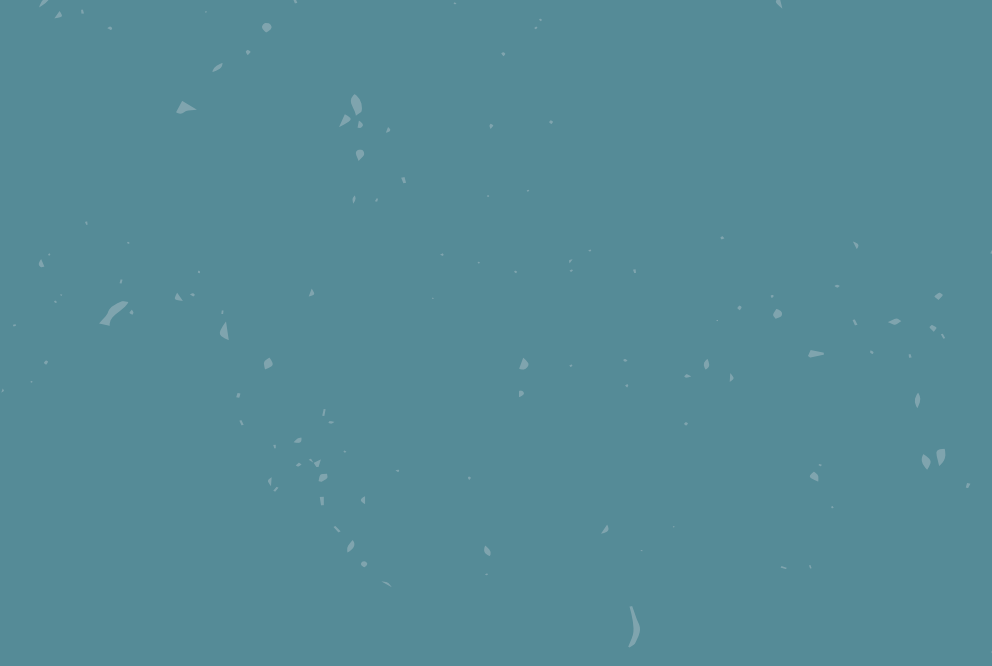

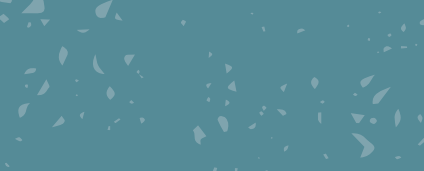




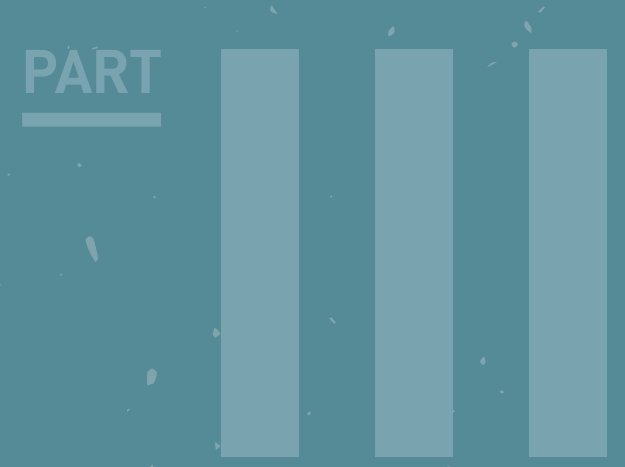

Symptomatic women and men with non-obstructed coronaries
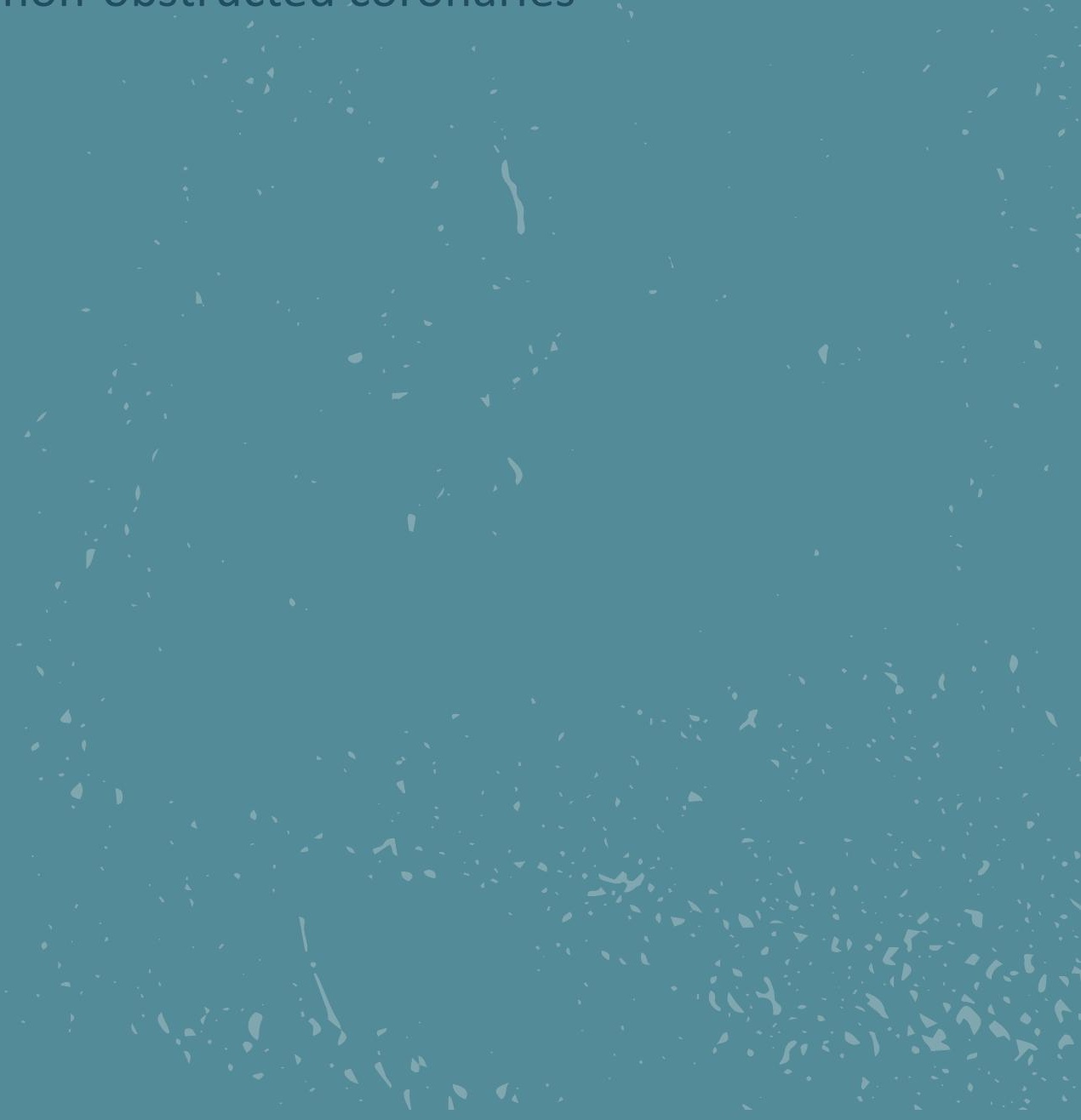


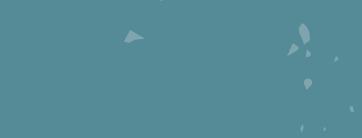

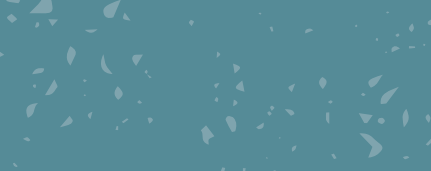

$\therefore \quad \therefore \quad \therefore \quad \therefore \quad \therefore$ CHAPTER
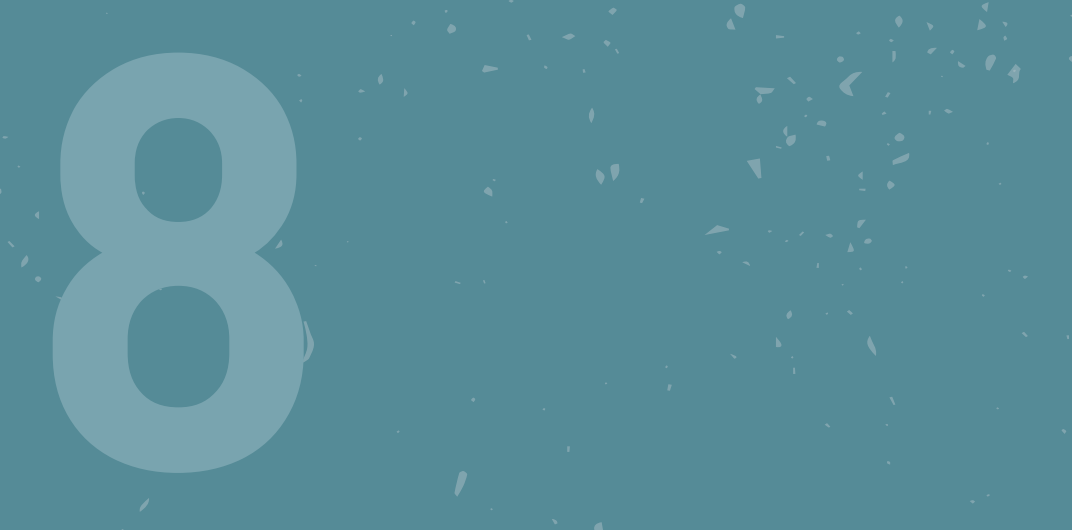
$\because \because \because$

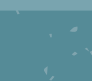

a

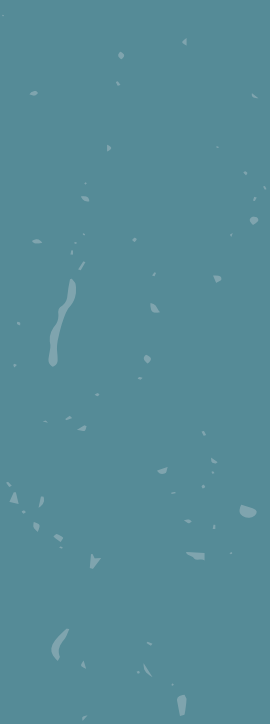

$\therefore$

i 


\section{Persistent symptoms and health needs of women and men with non-obstructed coronary arteries in the years following coronary angiography}

Frontiers in Cardiovascular Medicine 2021

Groepenhoff F, Eikendal ALM, Rittersma, Gijsberts CM, Asselbergs FW, Hoefer IE, Pasterkamp G, Rutten FH, Onland-Moret NC*, den Ruijter HM*

* These authors contributed equally 


\section{ABSTRACT}

The prognosis of women and men with persistent anginal complaints and nonobstructed coronary arteries is worse compared with asymptomatic women and men. The increased healthcare burden in the hospital due to repeated coronary angiography in these women and men has been documented, yet little is known about the percentage of women and men who remain symptomatic and under care of the general practitioner in the years following a coronary angiographic outcome of nonobstructed coronary arteries. From the Utrecht Coronary Biobank study, including individuals who underwent a coronary angiography from 2011 to 2015 ( $n=2,546$, $27 \%$ women), we selected women and men with non-obstructed coronary arteries ( $n=687,39 \%$ women). This population was linked to the Julius General Practitioners Network (JGPN); a database with routine care data of general practitioners. For every individual with non-obstructed coronary arteries, we selected an asymptomatic nonreferred age-, sex- and general practitioner-matched individual from the JGPN. We compared the healthcare consumption of men and women with non-obstructed coronary arteries to these matched individuals. The McNemar's test was used for pairwise comparison, and sex differences were assessed using stratified analyses. The prevalence of non-obstructed coronary arteries was higher in women as compared with men (39\% vs. 23\%). During a median follow-up of 7 years [IQR $6.4-8.0$ ], $89 \%$ of the individuals with non-obstructed coronary arteries (91\% women and $87 \%$ men) visited their general practitioner for one or more cardiovascular consultations. This was only $34 \%$ in the matched individuals ( $89 \%$ vs. $34 \%, p<0.001$ ). The consultations were most often for angina (equivalents) $(57 \%$ vs. $11 \%, p<0.001)$ and heart failure $(10 \%$ vs. $2 \%, p=0.015)$. In addition, individuals with non-obstructed coronaries at coronary angiography more often consulted the general practitioner for psychosocial complaints ( $31 \%$ vs. $15 \%, p=0.005)$. Findings were similar for women and men. In conclusion, a coronary angiographic outcome of non-obstructed coronary arteries is more common in women than in men. In the years following the coronary angiography, the majority of the population remains symptomatic. Both women and men with non-obstructed coronary arteries had higher health needs for angina, heart failure and psychosocial complaints than matched asymptomatic individuals. 


\section{INTRODUCTION}

Women with persistent complaints with non-obstructed coronary arteries have a poor prognosis (1). Recent evidence points to alternative mechanisms of cardiac ischemia that are often not detected during coronary angiography. Vasospastic angina and coronary microvascular disease may explain persistent symptoms, and the predisposition for heart failure with preserved ejection fraction (HFpEF) (2-4).

Relatively more women than men undergoing coronary angiography have nonobstructed coronary arteries (5). Specifically, the percentage of women undergoing coronary angiography that are referred back to the general practitioner with the outcome non-obstructed coronary arteries is even twice the percentage of men (6). As such, non-obstructed coronary arteries is mainly considered a 'women's problem' (5) and studies have been performed mostly in women (7) hampering a comparison with men (8). Furthermore, most studies focused selectively on women with persisting symptoms, not on all women with non-obstructed coronary arteries, that is, also including those without persisting anginal complaints $(1,7)$.

Prior studies on the outcome of these women were mainly investigating major adverse cardiovascular events as registered in the hospital setting $(9,10)$. In the Women's Ischemia Syndrome Evaluation (WISE) study, over $15 \%$ of women with non-obstructed coronary arteries underwent a second coronary angiography during 5 years of follow up (11). An European study evaluating both women and men undergoing coronary angiography with non-obstructed coronary arteries showed similar results in both sexes, namely more hospitalizations, repeat coronary angiography and overall more consultations in primary care as compared with asymptomatic individuals from the general population (12). No information was available on the reasons for these consultations in primary care. Thus, even though women and men with nonobstructed coronary arteries may not experience a hard endpoint, they may suffer from persistent symptoms and associated morbidities that affect health-related quality of life (11). In this study we assessed the percentage of women and men with non-obstructed coronary arteries who remain symptomatic in the years following the coronary angiography by linkage to a general practitioner's registry. In addition, we assessed their health need by comparing all cardiovascular and psychosocial primary care consultations to matched asymptomatic individuals. 


\section{MATERIALS AND METHODS}

\section{Patient population}

The UCORBIO cohort has been extensively described elsewhere (8). In short, UCORBIO comprises 2,546 consecutive routine care participants that underwent a coronary angiography between 2011 and 2015 at the UMC Utrecht. The study was approved by the Medical Ethics Committee of the UMC Utrecht (reference number 11-183), all participants provided written informed consent and the study conformed to the Declaration of Helsinki. Standardized electronic case record files were completed at baseline (containing age, sex, cardiovascular risk factors, indication for and findings from coronary angiography, medication use, treatment initiated after coronary angiography). Significant coronary artery disease was defined as $>50 \%$ stenosis, nonobstructive coronary artery disease as $<50 \%$ stenosis and/or wall irregularities and no coronary artery disease as normal coronary arteries. For this study we only analyzed data of women and men with non-obstructed coronary arteries consisting of the group of no and non-obstructed coronary arteries ( $n=687 ; 267$ (39\%) females) who consented to linkage of their data (Figure 1).

Follow up information on persisting symptoms and primary care consultations was obtained by linkage to the Julius General Practitioners Network (JGPN) database (13). Linkage was performed by a trusted third party using anonymous pseudo-identification to ensure protection of the privacy of the patients during linkage. The JGPN database comprises routine care data of individuals registered with participating general practitioners from the vicinity of Utrecht, The Netherlands. Because the catchment area for the UCORBIO patients was larger than the vicinity of Utrecht, only 139 (20\%) of the 687 UCORBIO patients could be identified within the JGPN database. We restricted our analyses to these 139 patients ( $n=53$ (38\%) women). Those identified within the JGPN database did not significantly differ from the remaining UCORBIO patients that could not be linked to JGPN (Supplementary Table 1). Follow-up information was available for a median of 7 years [IQR, 6.4-8.0].

\section{Comparison population}

The JGPN is a representative sample of the Dutch population; all Dutch citizens are registered with a general practitioner, except those living in a nursing home (12). We defined the entry date in this cohort for the UCORBIO individual with non-obstructed coronary arteries as the day of coronary angiography. For every individual with nonobstructed coronary arteries, we selected a matched individual registered in the JGPN who did not have symptoms on the entry date of the individual with non-obstructed coronary arteries. The women and men with non-obstructed coronary arteries were 


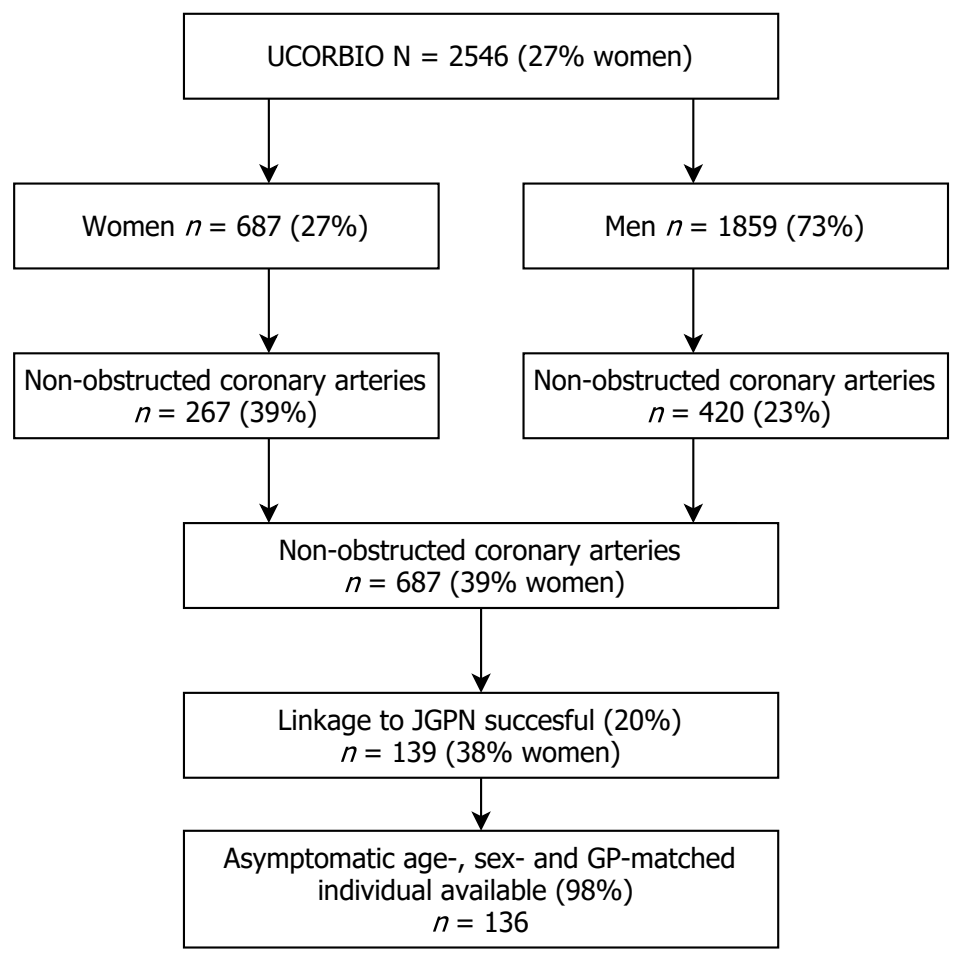

Figure 1. Flowchart of selection of the study population

matched to the asymptomatic JGPN individuals on age (in 5-year strata), sex, and general practitioner (1:1). Because a general practitioner's practice has on average 2,500 people enlisted, asymptomatic individuals within these strict selection criteria were limited. Furthermore, as every unique individual could only be selected once, for three individuals with non-obstructed coronary arteries an adequate asymptomatic individual was not available, leaving 136 (98\%) matched pairs.

\section{Data description}

All contacts between enlisted patients and their general practitioner were extracted from the general practitioner's electronical medical file consisting of all primary care consultations. The general practitioner classifies a diagnosis and/or main symptom according to the International Classification for Primary Care (ICPC) coding system (13) and labels every contact according to this ICPC coding system. Information on primary care use was collected similar for both women and men with non-obstructed coronary arteries as asymptomatic individuals. We restricted the extracted information to two domains; (suspected) cardiovascular disease and psychosocial problems, either 
labeled with ("K") in the ICPC coding system in case of cardiovascular and (" $\mathrm{P}$ ") for psychosocial problems. For the " $K$ " diagnoses and an additional single " $R$ " code for shortness of breath (R02), we further sub-analyzed these for chest pain or pressure (K01, K02), angina pectoris (K74), ischemic heart disease (IHD) (K75 and K76), shortness of breath (R02) and heart failure with preserved or reduced ejection fraction (K77). For some analyses we combined chest pain or pressure (K01, K02), angina pectoris (K74), ischemic heart disease (IHD) (K75 and K76) and shortness of breath (R02) to a single combined variable "angina (equivalent)". As the per-complaint analyses only focused on the most prevalent "K" diagnoses as mentioned above (K01, $\mathrm{K} 02, \mathrm{~K} 74, \mathrm{~K} 75, \mathrm{~K} 76, \mathrm{~K} 77$ ) and not on all possible "K" ICPC codes, the complete "cardiovascular disorders" count will include more complaints and therefore be higher than the sum of the consultations for selected ICPC codes; if an individual consulted the general practitioner for palpitations, this consultation is counted in the "cardiovascular disorders" count but not in the per-complaint analyses. Furthermore, we counted per ICPC code whether there was 'at least one' contact with the general practitioner as described above, but did not investigate the number of contacts per ICPC code. We evaluated all complaints separately and per complaint; if an individual consulted the general practitioner for both chest discomfort and shortness of breath during the follow-up period both were counted in the per-complaints analyses. Therefore, the total number of consultations can be higher than the number of participants analyzed in this study.

\section{Statistical analyses}

Continuous variables that were normally distributed were reported as means with standard deviation, whereas categorical variables were expressed as numbers and percentages. The pairwise McNemar's test was used for the comparison of proportions for cardiovascular or psychosocial consultations between women and men with nonobstructed coronary arteries and asymptomatic individuals. Sex differences were assessed by performing stratified analyses. A P-value $<0.05$ was considered statistically significant. All statistical analyses were performed using RStudio, version 3.5.2 (www. r-project.org).

\section{RESULTS}

\section{Baseline characteristics of women and men with non-obstructed coronary arteries}

Non-obstructed coronary arteries were more common in women than in men that underwent coronary angiography in the UCORBIO study (39\% women and $23 \%$ in men). Within the non-obstructed coronary artery population, the total proportion of women and men was $39 \%$ versus $61 \%$ (Figure 1 ). Baseline characteristics of the study 
population of women and men with non-obstructed coronary arteries are presented in Table 1. On average, these women were 3 years older than men with non-obstructed coronary arteries. Furthermore, comorbidities and risk factors are prevalent in both women and men (Table 1). Information on medication use can be found in the Supplementary material (Supplementary Table 2). Women were more likely to be non-smoking, to have hypertension at baseline and to be diagnosed with type 2 diabetes than men. However, these differences were not statistically significant. The prevalence of other established risk factors was comparable between women and men with non-obstructed coronary arteries. In men, even though not statistically significant, a history of myocardial infarction was more often documented than in women. The indication to perform coronary angiography as documented by the cardiologist was most often stable coronary artery disease in both sexes. In both sexes, a non-significant obstruction in one of the epicardial coronaries was more common than no obstruction at all (Table 1).

Table 1. Baseline characteristics of women and men with non-obstructed coronary arteries

\begin{tabular}{|c|c|c|c|}
\hline Characteristics & $\begin{array}{r}\text { Women } \\
\mathrm{N}=53\end{array}$ & $\begin{array}{l}\text { Men } \\
\mathrm{N}=86\end{array}$ & p-value \\
\hline Mean age (SD) - years & $66(13)$ & $63(12)$ & 0.08 \\
\hline Mean body mass index (SD) & $26.7(6)$ & $26.6(5)$ & \\
\hline Smoking, n (\%) & & & 0.065 \\
\hline Nonsmoker & $37(74)$ & $43(54)$ & \\
\hline Former smoker & $8(16)$ & $25(31)$ & \\
\hline Active smoker & $5(10)$ & $12(15)$ & \\
\hline Hypertension, $\mathrm{n}(\%)$ & $32(60)$ & $44(51)$ & 0.376 \\
\hline Diabetes, n (\%) & $12(23)$ & $14(16)$ & 0.477 \\
\hline Hypercholesterolemia, n (\%) & $22(42)$ & $39(45)$ & 0.789 \\
\hline Reduced kidney function, $\mathrm{n}(\%)$ & $1(2)$ & $2(2)$ & 1.000 \\
\hline Chest pain, $\mathrm{n}(\%)$ & $25(64)$ & $30(50)$ & 0.241 \\
\hline Shortness of breath, $\mathrm{n}(\%)$ & $23(59)$ & $20(33)$ & 0.021 \\
\hline LVEF $<50 \%, n(\%)$ & $3(7)$ & $11(18)$ & 0.178 \\
\hline COPD, n (\%) & $5(9)$ & $6(7)$ & 0.843 \\
\hline Previous myocardial infarction, $\mathrm{n}(\%)$ & $7(13)$ & $22(26)$ & 0.126 \\
\hline \multicolumn{4}{|l|}{ Indication for coronary angiography, $\mathrm{n}(\%)$} \\
\hline Unstable angina & $7(13)$ & $11(13)$ & 0.872 \\
\hline Acute myocardial infarction & $4(8)$ & $4(5)$ & \\
\hline Stable CAD & $30(57)$ & $48(56)$ & \\
\hline Other & $12(23)$ & $23(27)$ & \\
\hline \multicolumn{4}{|l|}{ Outcome CAG, $\mathrm{n}(\%)$} \\
\hline No CAD & $23(43)$ & $19(22)$ & \\
\hline $\begin{array}{l}\text { Minor CAD } \\
\text { (wall irregularities/coronary obstruction(s) }<50 \% \text { ) }\end{array}$ & $30(57)$ & $67(78)$ & \\
\hline
\end{tabular}

LVEF, left ventricular ejection fraction; CAD, coronary artery disease; COPD, chronic obstructive pulmonary disease 


\section{Cardiovascular consultations after coronary angiography showed non-obstructed coronary arteries}

During a median follow- up of 7 years [IQR 6.4-8.0], 123 (89\%) of the women and men with non-obstructed coronary arteries consulted their general practitioner. These consultations (once or more) were labelled for a cardiovascular disorder following the coronary angiography. The women and men with non-obstructed coronary arteries had significantly more consultations $(n=123(89))$ than the asymptomatic individuals ( $n=46(34 \%), p<0.001$ ). In $57 \%$ of the cases, the consultation was documented for angina or an angina equivalent (Table 2). There were no significant differences between women and men. The general practitioner was consulted by $62 \%$ of women and men with non-obstructed coronary arteries within the first year of follow up (68\% of women and $58 \%$ of men). A detailed analysis of the time between the coronary angiography and the cardiovascular and psychosocial consultations in the years following the coronary angiography can be found in Supplementary Figure 1.

Table 2. Cardiovascular consultations in women and men with non-obstructed coronary arteries vs. asymptomatic matched individuals

\begin{tabular}{lccc}
\hline & $\begin{array}{c}\text { Non-obstructed } \\
\text { coronaries (N=139) }\end{array}$ & $\begin{array}{l}\text { Asymptomatic matched } \\
\text { individuals (N=136) }\end{array}$ & P-value \\
\hline General practitioner consultation for & & & $<0.001$ \\
Cardiovascular disorders, n (\%) & $123(89)$ & $46(34)$ & $<0.001$ \\
No. of cardiovascular visits, median [IQR] & $17[5-52]$ & $0[0-8]$ & $<0.001$ \\
$\quad$ Angina (equivalent), $\mathrm{n}(\%)$ & $79(57)$ & $15(11)$ & $<0.001$ \\
$\quad$ Chest discomfort, $\mathrm{n}(\%)$ & $24(17)$ & $1(1)$ & $<0.001$ \\
$\quad$ Angina pectoris, $\mathrm{n}(\%)$ & $32(23)$ & $6(2)$ & $<0.001$ \\
$\quad$ Ischemic heart disease consult, $\mathrm{n}(\%)$ & $25(18)$ & $5(4)$ & 0.010 \\
$\quad$ Shortness of breath, $\mathrm{n}(\%)$ & $17(12)$ & $3(2)$ & 0.015 \\
\hline
\end{tabular}

\section{Psychosocial consultations}

The label " $\mathrm{P}$ " of the ICPC-coding system was used to study the incidence of primary care visits for psychosocial problems. Of the women and men with non-obstructive coronary arteries, 43 were documented under this label as compared to 21 of the asymptomatic individuals ( $31 \%$ vs. $15 \%, p=0.005$ ). In more detail, these consultations were mainly for anxiety, sleep or mood-related symptoms such as depression (Table 3). This higher prevalence of primary care consultations for psychosocial symptoms in the women and men with non-obstructed coronary arteries as compared with the asymptomatic individuals could not be explained by a higher prevalence of a history of psychological complaints (data not shown). 


\section{Subgroup-analyses for men and women}

When we repeated the analyses for women and men separately, similar results for both sexes were found (Table 4).

Table 3. Psychological consultations in women and men with non-obstructed coronary arteries vs. asymptomatic matched individuals

\begin{tabular}{|c|c|c|c|}
\hline & $\begin{array}{r}\text { Non-obstructed } \\
\text { coronaries } \\
(\mathrm{N}=139)\end{array}$ & $\begin{array}{r}\text { Asymptomatic } \\
\text { matched individuals } \\
(\mathrm{N}=136)\end{array}$ & P-value \\
\hline \multicolumn{4}{|l|}{ General practitioner consultation for } \\
\hline Psychological disorder, n (\%) & $43(31)$ & $21(15)$ & 0.005 \\
\hline Anxiety, n (\%) & $10(7)$ & $6(4)$ & \\
\hline Stress or work-related problems, n (\%) & $2(1)$ & $1(1)$ & \\
\hline Sleep disorder, $\mathrm{n}(\%)$ & $20(14)$ & $11(8)$ & \\
\hline Low mood, n (\%) & $21(15)$ & $6(4)$ & \\
\hline Depressive symptoms, $\mathrm{n}(\%)$ & $13(9)$ & $2(2)$ & \\
\hline Burn out, n (\%) & $6(4)$ & $2(2)$ & \\
\hline
\end{tabular}

Table 4. Cardiovascular consultations in women and men with non-obstructed coronary arteries vs. asymptomatic individuals

\begin{tabular}{|c|c|c|c|}
\hline & $\begin{array}{r}\text { Non-obstructed } \\
\text { coronaries }\end{array}$ & $\begin{array}{r}\text { Asymptomatic } \\
\text { matched individuals }\end{array}$ & P-value \\
\hline Women & $N=53$ & $N=52$ & \\
\hline \multicolumn{4}{|l|}{ General practitioner consultation for } \\
\hline Cardiovascular disorder, $\mathrm{n}(\%)$ & $48(91)$ & $17(33)$ & $<0.001$ \\
\hline Angina(equivalent), $\mathrm{n}(\%)$ & $31(59)$ & $6(12)$ & $<0.001$ \\
\hline Chest discomfort, $\mathrm{n}(\%)$ & $8(15)$ & $1(2)$ & \\
\hline Angina pectoris, $\mathrm{n}(\%)$ & $16(30)$ & $1(2)$ & \\
\hline Ischemic heart disease, $\mathrm{n}(\%)$ & $7(13)$ & $1(2)$ & \\
\hline Shortness of breath, $\mathrm{n}(\%)$ & $8(15)$ & $2(4)$ & \\
\hline Heart failure, n (\%) & $7(13)$ & $2(4)$ & \\
\hline Men & $N=86$ & $N=84$ & \\
\hline \multicolumn{4}{|l|}{ General practitioner consultation for } \\
\hline Cardiovascular disorder, $\mathrm{n}(\%)$ & $75(87)$ & $29(35)$ & $<0.001$ \\
\hline Angina(equivalent), n (\%) & $48(56)$ & $9(11)$ & $<0.001$ \\
\hline Chest discomfort, $\mathrm{n}(\%)$ & $16(19)$ & $0(0)$ & \\
\hline Angina pectoris, $\mathrm{n}(\%)$ & $16(19)$ & $2(2)$ & \\
\hline Ischemic heart disease, $\mathrm{n}(\%)$ & $18(21)$ & $5(6)$ & \\
\hline Shortness of breath, $n(\%)$ & $9(11)$ & $3(4)$ & \\
\hline Heart failure, $\mathrm{n}(\%)$ & $7(8)$ & $1(1)$ & \\
\hline
\end{tabular}




\section{Timing of general practitioner consultations in women and men with non-obstructed coronaries}

There is no clear pattern in the timing of the cardiovascular consultations of women and men with non-obstructed coronary arteries (Figure 2). Yet, the difference in the number of consultations for cardiovascular and psychosocial consultations during follow-up between individuals with non-obstructed coronary arteries (Figure 2, panel A, respectively, panel B) and asymptomatic matched individuals (Figure 2, panel C, respectively, panel D) is striking. Detailed timing of consultations is documented in the supplemental material (Supplementary figure 1).

\section{DISCUSSION}

Our study shows that the majority of both women and men with non-obstructed coronary arteries remain symptomatic in the years following the coronary angiography. They consult their general practitioner more often for cardiovascular and psychosocial care than matched asymptomatic individuals. Although the prevalence of nonobstructed coronary arteries is higher among women (6), our data does not indicate that there are differences between women and men in health needs in the years following coronary angiography. Notably, angina equivalent symptoms were more seen, but also a high burden of consultations for psychosocial symptoms was recorded, as compared with asymptomatic women and men.

Previous studies highlighted that individuals with non-obstructed coronary arteries were not as healthy as previously thought (14-16). Our results support these findings, as up to $90 \%$ of these individuals remain symptomatic, are likely to experience psychological issues and pursue medical care for these complaints, mostly at the general practitioner.

Although the absolute numbers are small, women and men with non-obstructed coronary arteries seem more prone to developing heart failure. This has been described before and a likely pathophysiological explanation for this phenomenon might be underlying microvascular disease (2). While the prevalence of microvascular disease has shown to be high in symptomatic women without obstructive coronary artery disease (16), our data do not indicate that men have less symptoms of heart failure in the years following angiography. Another possible explanation could be epicardial vasospasm, which is not regularly assessed during coronary angiography but is known to be a frequent cause of anginal complaints in the absence of obstructive coronary artery disease $(3,4)$. Yet, vasospastic angina as an isolated pathology does not seem to be accompanied by a poor prognosis (17). 


\section{Distribution of consultations per participant over time}

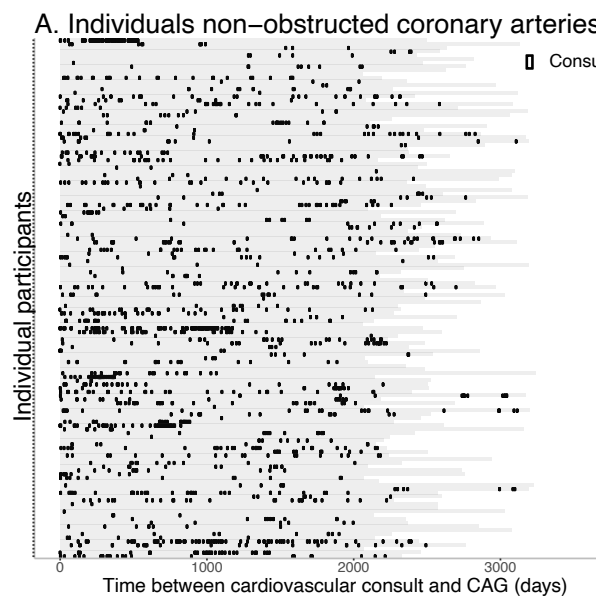

C. Asymptomatic matched individuals
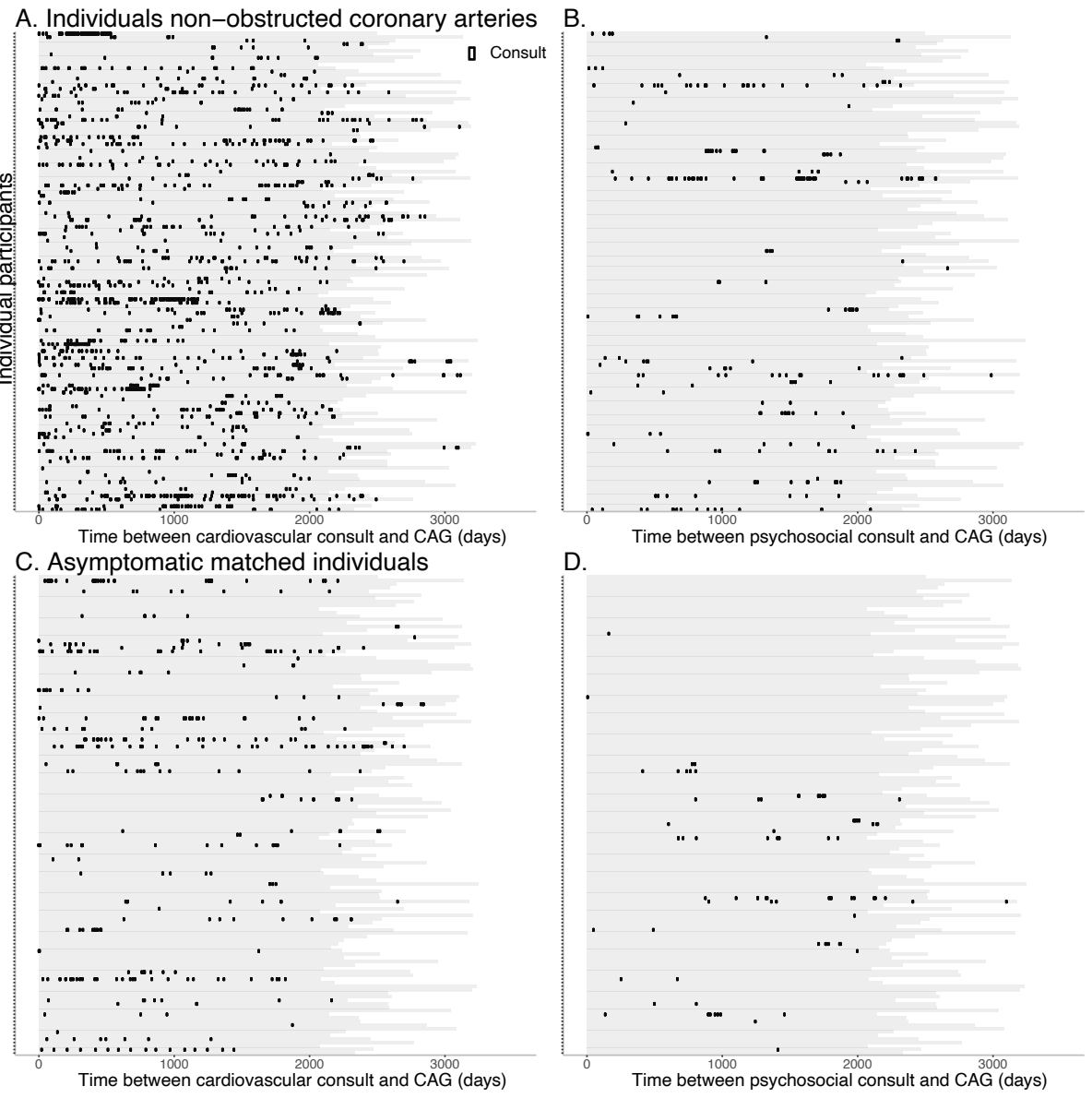

Figure 2. Visualization of cardiovascular (left) and psychological (right) consultations during follow-up after coronary angiography in women and men with non-obstructed coronary arteries (panel A, respectively, B) and matched asymptomatic men and women (panel C, respectively, D). Each horizontal bar represents the followup period in days of one individual participant and each dot represents a general practitioner consultation

In addition to cardiovascular complaints, the proportion of women and men that consult their general practitioner for psychological complaints is substantial. Although we were unable to assess causality due to the observational nature of this study, the association between psychosocial burden and non-obstructed coronary arteries seems to be strong in both women and men. These results are in line with previous research showing depression to be related to both the development as well as adverse outcomes of coronary artery disease (18). Even though previous research suggests 
the risk might be higher in females as compared to males $(19,20)$, we do not find evidence for this in our study. However, due to limited power, we cannot exclude or confirm sex differences in psychological complaints in these patients. The high burden of psychological complaints in this specific group of patients warrants urgent attention, both in clinical care as well as in research.

It is established that women and men with non-obstructed coronary arteries and prolonged anginal symptoms are at risk for (other) cardiovascular diseases, and with a rather poor prognosis (1). To date, it was unknown how large the population "at risk" is. This study has provided evidence that the number of patients with prolonged or recurrent symptoms suggestive of cardiac pathology is high in both sexes.

Our results suggest that the healthcare burden and costs are substantial, due to repeated general practitioner visits, the possible additional diagnostic tests that are performed and the economic burden, given that the majority of this population is still at working age. In the WISE study, the costs of symptom-driven care in women with persistent angina was reported to be almost 750,000 dollars per year (10). Even though healthcare costs cannot be compared between countries, it does provide insight in the extensive costs that are associated with symptomatic women and men with non-obstructed coronary arteries. Based on nation-wide reports, on average $20 \%$ of the patients who consult the cardiologist for chest discomfort the first time, at least a functional or anatomical investigation is performed, including over 10,000 coronary angiographies each year (18). Extrapolating our results, in $27 \%$ of these coronary angiographies non-obstructed coronary arteries would be the result of whom $90 \%$ (i.e. $n>2000$ ), would return to the general practitioner with prolonged complaints.

\section{Strengths and Limitations}

The strengths of this study are the fact that we have compared groups of the same source population and we included both women and men. Also, as we evaluated all women and men in whom non-obstructed coronary arteries were found, instead of selecting only those with prolonged complaints, we could evaluate the magnitude of the proportion of women and men with non-obstructed coronary arteries that remained symptomatic. Furthermore, we studied the health needs for both cardiovascular and psychological issues for a median duration of seven years. This study also has a number of limitations. Our study population did not undergo further coronary function assessment to assess whether microvascular disease or vasospasm was the underlying cause; extensive invasive testing protocols on women and men suffering from ischemia with non-obstructed coronary arteries have been recently 
established by the coronary vasomotor working group (COVADIS) (19), guiding when and how to evaluate coronary vascular dysfunction or vasospasm in these patients. These additional tests are currently being implemented in clinical care. Randomized trials assessing feasibility and value of these tests in improvement of quality of life and angina in these women and men (20) show promising results, possible due to improved perception of disease and/or tailored medical treatment. As the data was collected during regular care, we only had access to the data as documented by the general practitioner. Thus, we had to rely on ICPC codes to determine the prevalence of complaints. However, since general practitioners in the Netherlands are trained to only allocate a specific code to a patient if they are reasonably sure of their diagnosis, these codes can be used with a reasonably high certainty (21). Only $20 \%$ of the eligible women and men with non-obstructed coronary arteries in the UCORBIO database could be identified within the JGPN database. This selection was "at random"; as the women and men identified within the JGPN did not differ from the not-linked UCORBIO women and men, we can exclude selection bias. Yet, the power to perform detailed subgroup analyses was low and we could only compare the most prevalent complaints sex-stratified. Finally, as we performed our study in one center with a relatively homogeneous Caucasian population, our results might not be generalizable to other ethnic populations undergoing coronary angiography.

\section{CONCLUSIONS}

The diagnosis of non-obstructed coronary arteries is more common in women than in men undergoing coronary angiography. The majority of the angina population without obstructive CAD remains symptomatic following coronary angiography. They had over two-fold higher health needs for angina, heart failure and psychosocial complaints compared to controls. There were no differences between men's and women's health needs following coronary angiography without obstructive CAD. 


\section{REFERENCES}

1. Gulati M, Cooper-DeHoff RM, McClure C, Johnson BD, Shaw LJ, Handberg EM, et al. Adverse cardiovascular outcomes in women with nonobstructive coronary artery disease: a report from the Women's Ischemia Syndrome Evaluation Study and the St James Women Take Heart Project. Archives of internal medicine. 2009;169(9):843-50.

2. Paulus WJ, Tschöpe C. A novel paradigm for heart failure with preserved ejection fraction: comorbidities drive myocardial dysfunction and remodeling through coronary microvascular endothelial inflammation. Journal of the American College of Cardiology. 2013;62(4):263-71.

3. Prinzmetal M, Kennamer R, Merliss R, Wada T, Bor N. Angina pectoris I. A variant form of angina pectoris: preliminary report. The American journal of medicine. 1959;27(3):375-88.

4. Aziz A, Hansen HS, Sechtem U, Prescott E, Ong P. Sex-related differences in vasomotor function in patients with angina and unobstructed coronary arteries. Journal of the American College of Cardiology. 2017;70(19):2349-58.

5. Shaw LJ, Bugiardini R, Merz CNB. Women and ischemic heart disease: evolving knowledge. Journal of the American College of Cardiology. 2009;54(17):1561-75.

6. Gijsberts CM, Gohar A, Ellenbroek GH, Hoefer IE, de Kleijn DP, Asselbergs FW, et al. Severity of stable coronary artery disease and its biomarkers differ between men and women undergoing angiography. Atherosclerosis. 2015;241(1):234-40.

7. Barsky L, Merz CNB, Wei J, Shufelt C, Handberg E, Pepine C, et al. Even "WISE-R?"-an Update on the NHLBISponsored Women's Ischemia Syndrome Evaluation. Current atherosclerosis reports. 2020;22(8):1-13.

8. Gijsberts CM, Santema BT, Asselbergs FW, de Kleijn DP, Voskuil M, Agostoni P, et al. Women undergoing coronary angiography for myocardial infarction or who present with multivessel disease have a poorer prognosis than men. Angiology. 2016;67(6):571-81.

9. de Heer EW, Palacios JE, Adèr HJ, van Marwijk HW, Tylee A, van der Feltz-Cornelis CM. Chest pain, depression and anxiety in coronary heart disease: Consequence or cause? A prospective clinical study in primary care. Journal of Psychosomatic Research. 2020;129:109891.

10. Kim Y, Soffler M, Paradise S, Dziura J, Sinha R, Safdar B. Depression is associated with recurrent chest pain with or without coronary artery disease: a prospective cohort study in the emergency department. American heart journal. 2017;191:47-54.

11. Shaw LJ, Merz CNB, Pepine CJ, Reis S, Bittner V, Kip KE, et al. The economic burden of angina in women with suspected ischemic heart disease. Circulation. 2006;114(9):894-904.

12. Jespersen L, Abildstrom SZ, Hvelplund A, Madsen JK, Galatius S, Pedersen F, et al. Burden of hospital admission and repeat angiography in angina pectoris patients with and without coronary artery disease: a registry-based cohort study. PloS one. 2014;9(4):e93170.

13. Smeets HM, Kortekaas MF, Rutten FH, Bots ML, van der Kraan W, Daggelders G, et al. Routine primary care data for scientific research, quality of care programs and educational purposes: the Julius General Practitioners' Network (JGPN). BMC health services research. 2018;18(1):735.

14. Jespersen L, Hvelplund A, Abildstrøm SZ, Pedersen F, Galatius S, Madsen JK, et al. Stable angina pectoris with no obstructive coronary artery disease is associated with increased risks of major adverse cardiovascular events. European heart journal. 2011;33(6):734-44.

15. Shaw LJ, Merz CNB, Pepine CJ, Reis SE, Bittner V, Kip KE, et al. The economic burden of angina in women with suspected ischemic heart disease: results from the National Institutes of Health-National Heart, Lung, and Blood Institute-sponsored Women's Ischemia Syndrome Evaluation. Circulation. 2006;114(9):894-904.

16. Reis SE, Holubkov R, Smith AC, Kelsey SF, Sharaf BL, Reichek N, et al. Coronary microvascular dysfunction is highly prevalent in women with chest pain in the absence of coronary artery disease: results from the NHLBI WISE study. American heart journal. 2001;141(5):735-41.

17. Egashira K, Kikuchi Y, Sagara T, Sugihara M, Nakamura M. Long-term prognosis of vasospastic angina without significant atherosclerotic coronary artery disease. Japanese heart journal. 1987;28(6):841-9.

18. Nederland Zorginstituut, Verbetersignalement Pijn op de borst (verdenking) stabiele angina pectoris. https://www. zorginstituutnederland.nl/publicaties/rapport/2018/01/31/zinnige-zorg-verbetersignalement-\%E2\%80\%98pijnop-de-borst\%E2\%80\%992017. p. 80.

19. Ford TJ, Stanley B, Good R, Rocchiccioli P, McEntegart M, Watkins S, et al. Stratified medical therapy using invasive coronary function testing in angina: the CorMicA trial. Journal of the American College of Cardiology. 2018;72(23 Part A):2841-55. 
20. Ford TJ, Ong P, Sechtem U, Beltrame J, Camici PG, Crea F, et al. Assessment of vascular dysfunction in patients without obstructive coronary artery disease: why, how, and when. Cardiovascular Interventions. 2020;13(16):1847-64. 


\section{SUPPLEMENTARY MATERIAL}

Supplementary Table 1. Comparison of UCORBIO men and women with non-obstructed coronary arteries that could be identified in the JGPN database compared with those that could not be identified and therefore were not included in this study

\begin{tabular}{|c|c|c|c|}
\hline $\mathbf{n}$ & $\begin{array}{r}\text { Overall } \\
687\end{array}$ & $\begin{array}{r}\text { No JGPN } \\
548\end{array}$ & $\begin{array}{r}\text { JGPN } \\
139\end{array}$ \\
\hline Women, n (\%) & $267(38.9)$ & $214(39.1)$ & $53(38.1)$ \\
\hline Mean age (SD) - years & $62(12)$ & $62(12)$ & $64(12)$ \\
\hline \multicolumn{4}{|l|}{ Smoking, $\mathrm{n}(\%)$} \\
\hline Non smoker & $385(59.7)$ & $305(59.2)$ & $80(61.5)$ \\
\hline Former smoker & $158(24.5)$ & $125(24.3)$ & $33(25.4)$ \\
\hline Active smoker & $102(15.8)$ & $85(16.5)$ & $17(13.1)$ \\
\hline Hypertension, n (\%) & $359(52.3)$ & $283(51.6)$ & $76(54.7)$ \\
\hline Diabetes, n (\%) & $112(16.3)$ & $86(15.7)$ & $26(18.7)$ \\
\hline Hypercholesterolemia, n (\%) & $287(41.8)$ & $226(41.2)$ & $61(43.9)$ \\
\hline Mean body mass index (SD) & $26.2(4.7)$ & $26.1(4.7)$ & $26.6(5.0)$ \\
\hline Kidney disease, n (\%) & $23(3.3)$ & $20(3.6)$ & $3(2.2)$ \\
\hline Chest pain, $\mathrm{n}(\%)$ & $239(43.9)$ & $184(41.3)$ & $55(55.6)$ \\
\hline Shortness of breath, $\mathrm{n}(\%)$ & $187(34.3)$ & $144(32.3)$ & $43(43.4)$ \\
\hline LVEF $<50 \%, n(\%)$ & $105(18.5)$ & $91(19.7)$ & $14(13.2)$ \\
\hline COPD, n (\%) & $60(8.7)$ & 49 ( 8.9) & $11(7.9)$ \\
\hline Previous MI, n (\%) & $119(17.3)$ & $90(16.4)$ & $29(20.9)$ \\
\hline \multicolumn{4}{|l|}{ Indication, $\mathrm{n}(\%)$} \\
\hline UAP & $49(7.1)$ & $31(5.7)$ & $18(12.9)$ \\
\hline Myocardial infarction & $41(6.0)$ & $33(6.0)$ & $8(5.8)$ \\
\hline Other & $194(28.2)$ & $159(29.0)$ & $35(25.2)$ \\
\hline Stable CAD & 403 (58.7) & $325(59.3)$ & 78 (56.1) \\
\hline $\begin{array}{l}\text { Minor CAD (\%) (wall irregularities/coronary } \\
\text { obstruction(s) <50\%) }\end{array}$ & $464(67.5)$ & $367(67.0)$ & $97(69.8)$ \\
\hline
\end{tabular}

LVEF, left ventricular ejection fraction; $\mathrm{MI}$, myocardial infarction; UAP, unstable angina pectoris; CAD, coronary artery disease; CAG, coronary angiography. 
Supplementary Table 2. Baseline medication use of women and men with non-obstructed coronary arteries stratified by sex

\begin{tabular}{lcc}
\hline $\mathbf{n}$ & Women & Men \\
$\mathbf{8 6}$
\end{tabular}

$\mathrm{ACE}$, angiotensin converting enzyme; $\mathrm{ARB}$, angiotensin receptor blocker 

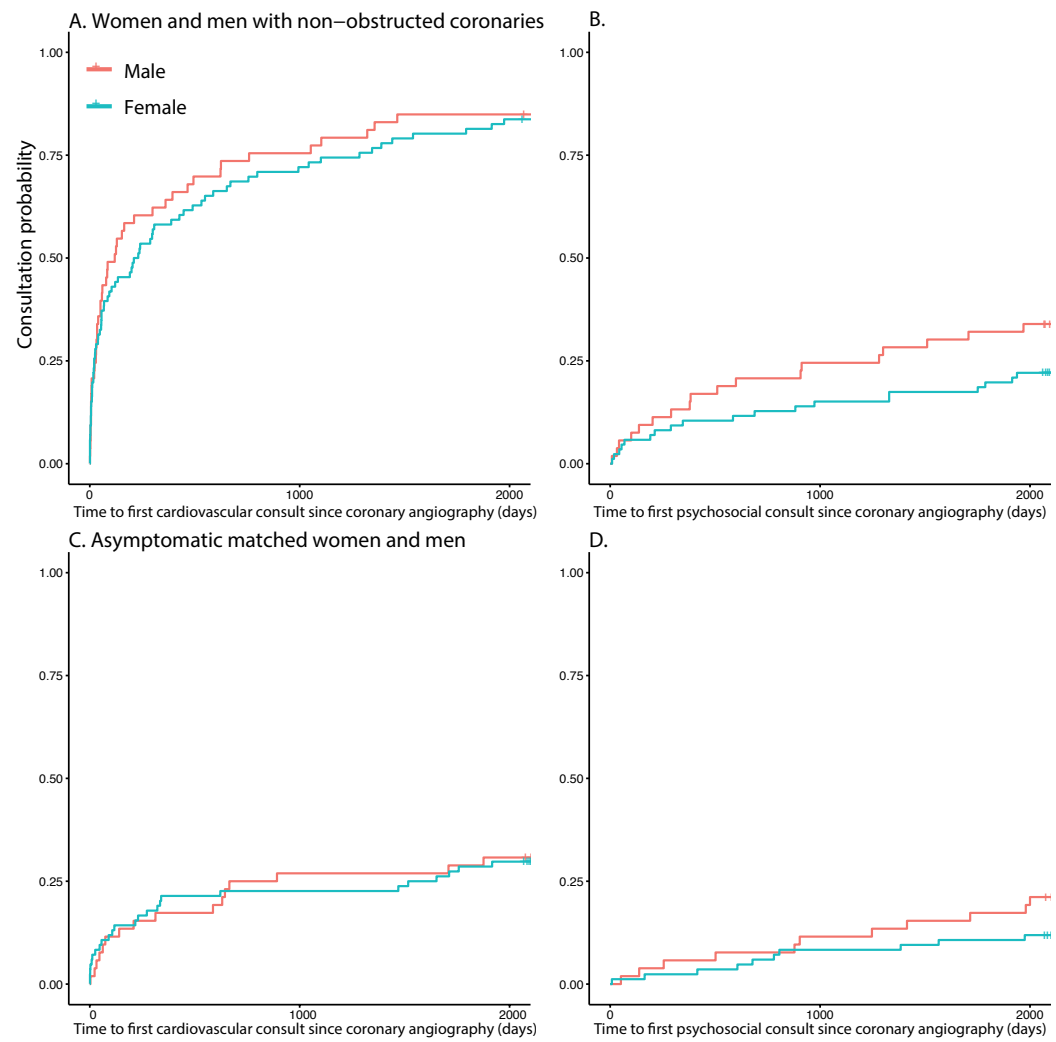

Supplementary Figure 1. Timing of first cardiovascular (left) and psychological (right) consultations at the general practitioner for women and men with non-obstructed coronaries (above) following coronary angiography and matched individuals (below) 


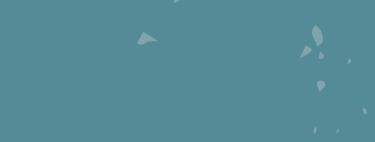

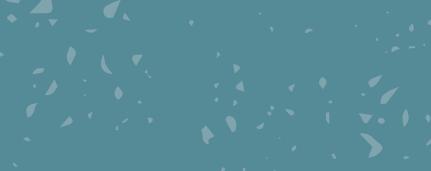

$\therefore \quad \therefore \div ; \div$ CHAPTER
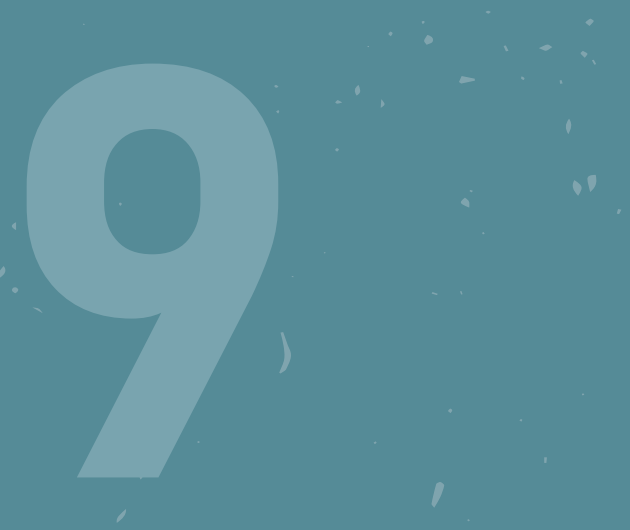

$\therefore-\therefore \vdots \vdots$
$-\because<\vdots$
$1 \div$

$\because \ldots \therefore$

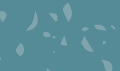

$\because$

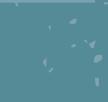

:

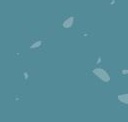

,

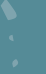

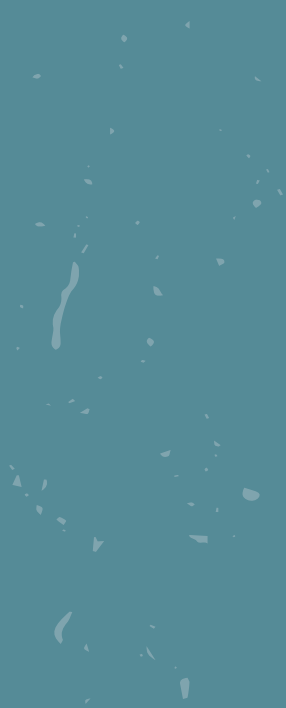




\section{Evaluation of non-invasive imaging parameters in coronary microvascular disease: a systematic review}

BMC Medical Imaging 2021

Groepenhoff F*, Klaassen RGM*, Valstar GB, Bots SH, Onland-Moret NC, den Ruijter HM, Leiner T, Eikendal ALM 


\begin{abstract}
Coronary microvascular dysfunction (CMD) is an important underlying cause of angina pectoris. Currently, no diagnostic tool is available to directly visualize the coronary microvasculature. Invasive microvascular reactivity testing is the diagnostic standard for $\mathrm{CMD}$, but several non-invasive imaging techniques are being evaluated. However, evidence on reported non-invasive parameters and cut-off values is limited. Thus, we aimed to provide an overview of reported non-invasive parameters and corresponding cut-off values for CMD. PubMed and Embase databases were systematically searched for studies enrolling patients with angina pectoris without obstructed coronary arteries, investigating at least one non-invasive imaging technique to quantify CMD. Methodological quality assessment of included studies was performed using QUADAS-2. Thirty-seven studies were included. Ten cardiac magnetic resonance (CMR) studies reported MPRI and nine positron emission tomography (PET) and eleven transthoracic echocardiography (TTE) studies reported CFR. Mean MPRI ranged from $1.47 \pm 0.36$ to $2.01 \pm 0.41$ in patients and from $1.50 \pm 0.47$ to $2.68 \pm 0.49$ in controls without CMD. Reported mean CFR in PET and TTE ranged from $1.39 \pm 0.31$ to $2.85 \pm$ 1.35 and $1.69 \pm 0.40$ to $2.40 \pm 0.40$ for patients, and $2.68 \pm 0.83$ to $4.32 \pm 1.78$ and $2.65 \pm 0.65$ to $3.31 \pm 1.10$ for controls, respectively. This systematic review summarized current evidence on reported parameters and cut-off values to diagnose CMD for various non-invasive imaging modalities. In current clinical practice, CMD is generally diagnosed with a CFR less than 2.0. However, due to heterogeneity in methodology and reporting of outcome measures, outcomes could not be compared and no definite reference values could be provided.
\end{abstract}




\section{BACKGROUND}

Patients with angina pectoris (AP) often do not show significant obstructive coronary artery disease (CAD) on coronary angiography (CAG) $(1,2)$. Consequently, a cardiac cause of AP complaints is frequently deemed unlikely. Yet, a significant fraction of these patients suffer from cardiac ischemia due to coronary microvascular dysfunction (CMD) (2-6), a condition associated with increased risk of adverse cardiovascular events. This emphasizes the importance of accurate diagnosis of $\operatorname{CMD}(2,7-11)$.

The Coronary Vasomotion Disorders International Study Group (COVADIS) determined the following criteria to diagnose CMD: presence of symptoms and objective documentation of myocardial ischemia, absence of obstructive CAD ( $<50 \%$ stenosis and/or fractional flow reserve $<0.8$ ) and confirmed reduced coronary flow reserve (CFR) (and/or inducible microvascular spasm). However, assessment of CMD remains challenging, as no tools are available to directly visualize the coronary microvasculature. In fact, the current golden standard to diagnose CMD is invasive measurement of CFR in epicardial arteries without functionally relevant stenosis (12). The CFR depicts the increase in coronary blood flow in response to vasoactive agents $(2,4,9)$ and provides indirect quantification of coronary microvascular blood flow $(5,13)$.

The invasive nature and high costs of coronary reactivity testing (CRT) initiated the search for a non-invasive alternative to diagnose CMD, including myocardial perfusion reserve index (MPRI) measured using cardiac magnetic resonance imaging (CMR), and CFR using positron emission tomography (PET) and transthoracic echocardiography (TTE) (13-16). Yet, the cut-off value for CFR to diagnose CMD differs between these modalities, is not well validated and, even though sex-differences in coronary physiology are known, the need for a sex-specific cut-off value remains under debate (17). To date, a CFR below 2.0 to 2.5 is deemed diagnostic for $\operatorname{CMD}(9,16,18-20)$.

The (dis)advantages of these non-invasive imaging techniques in the diagnosis of CMD have been discussed extensively before (21). However, it is unclear which outcome parameters and corresponding cut-off values should be used to diagnose CMD. As such, this systematic review aims to provide an overview of currently reported reference and cut-off values for diagnosing CMD in a non-invasive manner. 


\section{METHODS}

\section{Search strategy}

On October 15, 2018 the PubMed and Embase databases were systematically searched for non-invasive imaging studies on CMD. The search was updated on November 1, 2020. Studies were considered for eligibility without date restriction. The search terms and synonyms of 'coronary microvascular dysfunction', 'nonobstructive coronary disease' and 'imaging', including the imaging modalities CMR, PET and TTE were used. A broad search strategy was performed as studies on CMD are limited and nomenclature of CMD is not standardized. Therefore, search terms were searched for in 'All Fields'. The detailed search strategy is provided in Additional file 1: Search Strategy.

\section{Study selection}

To assess eligibility, the results from the literature search were initially screened by title and abstract and subsequently for full text. Article selection and data extraction were performed independently by two reviewers (RGMK and FG). Observational studies and randomized controlled trials providing baseline outcome measurements were considered for inclusion.

Studies were included if they enrolled participants with AP (i.e. effort angina or anginal equivalents) and CAG or coronary computed tomography angiography confirmed absent or nonobstructive CAD (based on the definition described in the study protocol of the included studies), or healthy participants without prior history of cardiovascular disease or AP as a control group and reported the results of a non-invasive imaging method with use of pharmacological stress (i.e. flow parameters measured with either CMR, PET or TTE) to diagnose CMD.

Studies written in languages other than English or Dutch, exclusively consisting of participants with comorbidities, i.e. CAD, diabetes mellitus, aortic stenosis or cardiomyopathies, were excluded. Studies were excluded if outcomes were not reported as flow parameters, if patients were stratified according to the outcome of interest or if patient or control groups contained fewer than 10 participants.

\section{Quality assessment}

A methodological quality assessment was performed with the QUADAS-2 (Tool for the Quality Assessment of Diagnostic Accuracy Studies (22)). Studies were assessed for concerns of applicability ('low', 'high' or 'unclear') and for risk of bias ('low', 'high' or 'unclear') on four key domains (patient selection, index test, reference standard 
and flow and timing). The assessments per domain were combined into an overall risk of bias and concern of applicability.

\section{Data extraction and analysis}

The variables of interest were extracted using a standardized data collection form. Post-hoc evaluations within one clinical trial assessing the same imaging modality were considered as one study. Due to heterogeneity of the included studies, metaanalysis of the results was not possible.

\section{RESULTS}

\section{Search results}

The search yielded a total of 6,976 results, 2,568 studies in PubMed and 4,408 studies in Embase. Removal of duplicates resulted in 5,238 unique entries. After title and abstract screening, 443 possibly relevant studies were obtained. The full texts of these studies were screened to select those that met the inclusion criteria as provided in the methods section. One relevant study was obtained through cross-reference checking. Thirty-seven studies met the inclusion criteria and were included in the final analysis. The search and inclusion and exclusion of relevant studies are summarized in Figure 1. The characteristics of the included studies are summarized in Table 1. Quality assessment of included studies showed a clear description of the reference standard was not part of the study protocol in most of the included studies. The full assessment is provided in the supplemental material (Additional file 2: Table S1) (22).

\section{Demographic information}

The number of patients included in each study was generally small, with a median study population of 22 patients (range: 11 to $963,89 \%$ women) and median of 18 controls (range: 10 to 268, 33\% women). The mean age in patient groups ranged from $50.0 \pm 7.0$ to $66.0 \pm 10.0$ years of age and $35.3 \pm 3.9$ to $62.6 \pm 9.1$ years of age in control groups. The specific demographic information per study is summarized in Table 1 (printed at the end of this chapter).

\section{Flow parameters}

Different flow parameters were reported (Table 2). In CMR studies, the myocardial perfusion reserve index (MPRI) was most often reported. Other parameters reported were myocardial perfusion reserve (MPR) and CFR. MPR is defined as the ratio between the relative upslope of myocardial signal intensity (obtained with the use of gadolinium as contrast agent) during stress and rest. In contrast to MPR, the MPRI 
is corrected for left ventricular contrast signal intensity, allowing for a reduction in signal differences within the image and intra-individual level differences in signal intensity due to heart rate and blood pressure $(6,23,24)$. As such, MPRI is often the preferred outcome measure as it seems to be more accurate in quantifying coronary microvascular blood flow. In one study, CMR-derived CFR results were presented (25). CFR was calculated and measured in the exact same way as the MPR and can therefore be considered as a synonym of MPR.

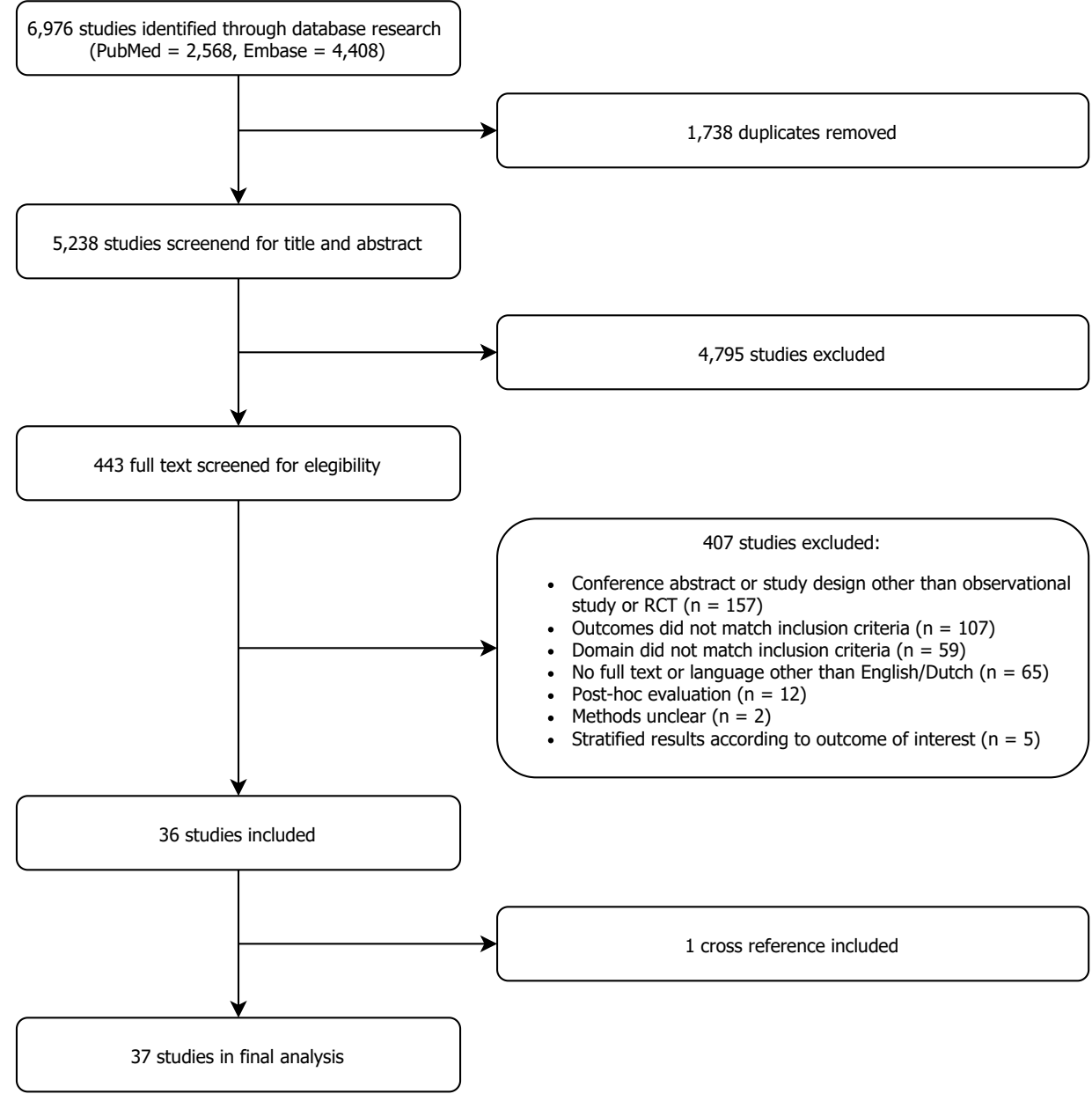

Figure 1. Flow chart study design $\mathrm{RCT}$, randomized controlled trial 
Table 2. Overview of outcome parameters considered in this systematic review

\begin{tabular}{|c|c|c|}
\hline Imaging method & Parameters & Definition \\
\hline \multirow[t]{3}{*}{$\begin{array}{l}\text { Cardiac magnetic } \\
\text { resonance imaging }\end{array}$} & Myocardial perfusion reserve index (MPRI) & $\begin{array}{l}\mathrm{MBF}_{\text {stress }} / \mathrm{MBF}_{\text {rest }} * \mathrm{LV} \text { contrast signal } \\
\text { intensity. }\end{array}$ \\
\hline & Myocardial perfusion reserve (MPR) & $\mathrm{MBF}_{\text {stress }} / \mathrm{MBF}_{\text {rest }}$ \\
\hline & $\begin{array}{l}\text { Coronary flow reserve (corrected for rate } \\
\text { pressure product) (CFR (corrected for RPP)) }\end{array}$ & $\mathrm{MBF}_{\text {stress }} / \mathrm{MBF}_{\text {rest }} *(\mathrm{HR} * \mathrm{SBP} / 10.000)$ \\
\hline \multirow[t]{3}{*}{$\begin{array}{l}\text { Positron emmission } \\
\text { tomography }\end{array}$} & $\begin{array}{l}\text { Coronary flow reserve (corrected for rate } \\
\text { pressure product) (CFR (corrected for RPP)) }\end{array}$ & $\mathrm{MBF}_{\text {stress }} / \mathrm{MBF}_{\text {rest }} *(\mathrm{HR} * \mathrm{SBP} / 10.000)$ \\
\hline & Myocardial perfusion reserve (MPR) & $\mathrm{MBF}_{\text {stress }} / \mathrm{MBF}_{\text {rest }}$ \\
\hline & Myocardial flow reserve (MFR) & $\mathrm{MBF}_{\text {stress }} / \mathrm{MBF}_{\text {rest }}$ \\
\hline \multirow{3}{*}{$\begin{array}{l}\text { Transthoracic } \\
\text { echocardiography }\end{array}$} & Coronary flow reserve (CFR) & $\mathrm{MBF}_{\text {stress }} / \mathrm{MBF}_{\text {rest }}$ \\
\hline & Coronary flow velocity reserve (CFVR) & $\mathrm{MBF}_{\text {stress }} / \mathrm{MBF}_{\text {rest }}$ \\
\hline & Coronary blood flow (CBF) & $\mathrm{MBF}_{\text {stress }} / \mathrm{MBF}_{\text {rest }}$ \\
\hline
\end{tabular}

$\mathrm{HR}$, heart rate; $\mathrm{LV}$, left ventricular; $\mathrm{MBF}_{\text {rest' }}$ myocardial blood flow in resting conditions; $\mathrm{MBF}_{\text {stres' }}$ myocardial blood flow during hyperemic circumstances; SBP, systolic blood pressure

In PET studies microvascular function was usually quantified with CFR. Other outcome parameters were MPR or myocardial flow reserve (MFR). MPR and MFR were calculated based on the same methods and measurements as CFR and could therefore be used interchangeably. CFR was defined as the ratio between hyperemic and resting myocardial blood flow (MBF) $(26,27)$ which was expressed in $\mathrm{ml} / \mathrm{min} / \mathrm{g}(28)$. CFR was often corrected for rate pressure product (RPP), defined as heart rate multiplied by systolic blood pressure and represents cardiac metabolic demand. This correction is recommended as it reduces variability in outcomes due to person-level differences in systolic blood pressure and heart rate $(2,27)$.

In TTE studies CFR was used. Similar to PET and CMR, a variety of equivalent terms were reported, namely CFR, coronary blood flow (CBF) and coronary flow velocity reserve (CFVR). CFR, CBF and CFVR were all defined as the ratio of peak stress and rest coronary blood flow velocities (CBFV), usually obtained by spectral Doppler measurements.

\section{CMR imaging}

CMR was used to diagnose CMD in 15 of the 37 included studies (Additional file 3: Table S2) $(6,15,23,25,29-39)$. CMR results are mostly expressed as the MPRI (n= 
11). The other outcome parameters mentioned were MPR and CFR $(n=5)$. One study assessed MPRI as well as MPR (29). Patient groups were globally comparable as all studies included patients with AP without CAD on CAG. Absolute mean transmural mean MPRI values in patient groups ranged from $1.47 \pm 0.36$ to $2.01 \pm 0.41$. In controls, mean MPRI ranged from $1.50 \pm 0.47$ to $2.68 \pm 0.49$. The results of CMR studies with MPRI as outcome parameter in patients and controls are summarized in Figure 2A.

\section{PET imaging}

A total of 13 studies used PET to quantify coronary microvascular function (Additional file 4: Table S3) $(16,35,40-50)$. In PET studies reporting mean CFR as outcome measure, mean CFR ranged from $1.39 \pm 0.31$ to $2.85 \pm 1.35$ in patient groups. In the control group, mean CFR ranged from $2.68 \pm 0.83$ to $4.32 \pm 1.78$. The results of PET studies with CFR as outcome parameter in patients and controls are summarized in Figure 2B.

\section{TTE imaging}

In 11 studies CMD was assessed by TTE (Additional file 5: Table S4) (16, 51-60). All studies calculated CFR as the ratio of basal and hyperemic diastolic flow velocity measured in the left anterior descending coronary artery (LAD). In the included TTE studies, patient groups were comparable with regard to inclusion of patients with AP and no or nonobstructive CAD on CAG (Table 1). Two RCTs were included, mentioning CFR at baseline. A mean CFR of $1.69 \pm 0.40$ to $2.40 \pm 0.40$ was found in patients with angina and no CAD on CAG, whereas healthy control subjects show a higher mean CFR of $2.65 \pm 0.65$ to $3.31 \pm 1.10$. An overview of the CFR outcomes of TTE studies in patients and controls is presented in Figure 2C.

\section{Sex differences}

Only one of the included studies compared outcomes between men and women. Sestito et al. (56) determined CBF (defined as the ratio of diastolic CBF velocity at peak stress and rest) using TTE in 71 patients diagnosed with CMD (48 women, 68\%). No significant difference in CBF was found between men and women (CBF $2.09 \pm 0.60$ and $2.03 \pm 0.50$, respectively). Furthermore, the proportion of women as compared to men in the patient groups was much larger (89\%) as compared with the control population (33\%). 

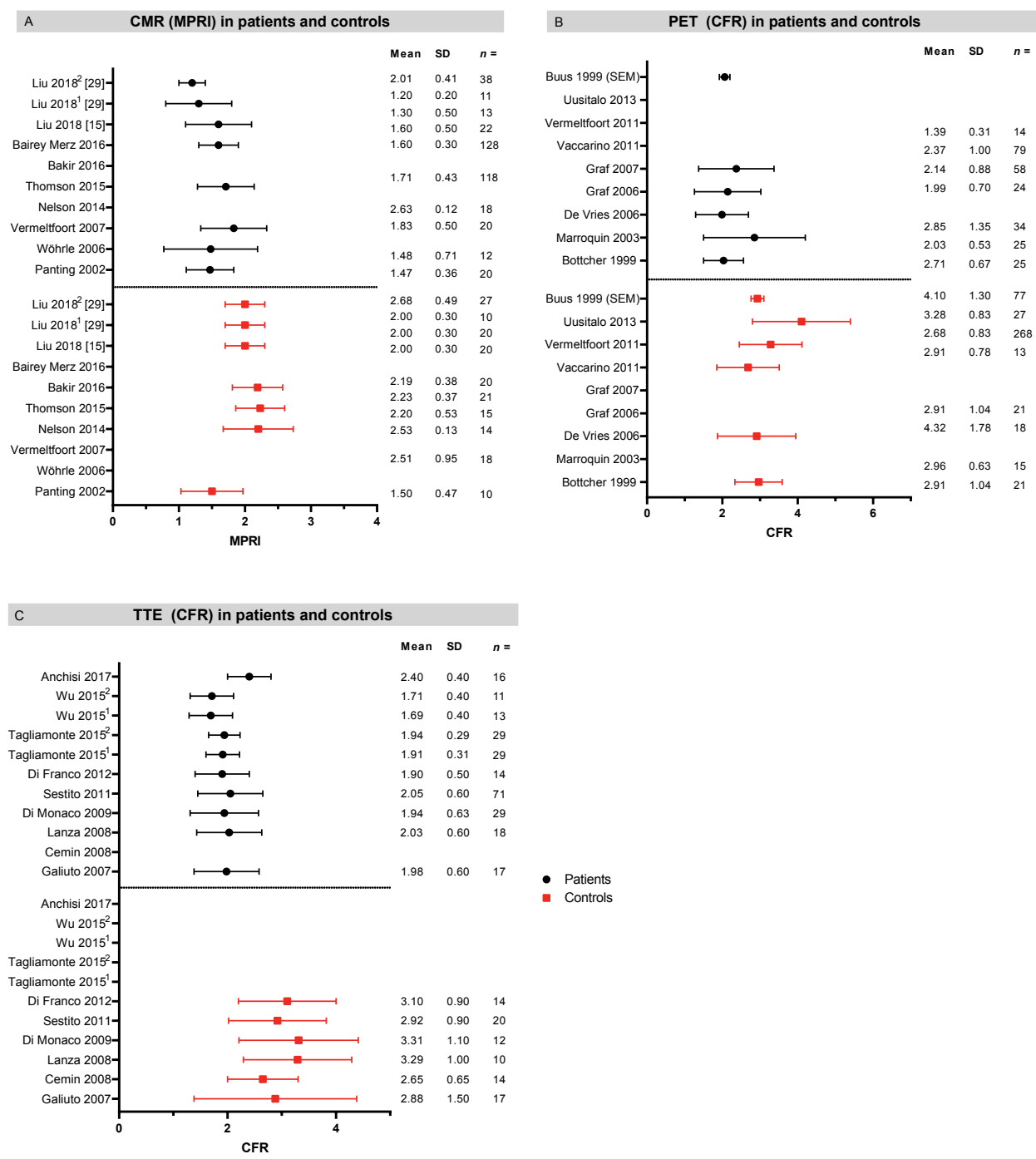

Figure 2. Overview of study outcomes presented as mean \pm SD in patients and controls for MPRI by CMR (A), CFR by PET (B) and CFR by TTE (C). For CMR. Error bars are not shown for some studies as some only assessed patient or control subjects. Studies with multiple patient or control groups are indicated by numbers $\mathrm{CFR}$, coronary flow reserve; $\mathrm{CMR}$, cardiac magnetic resonance imaging; MPRI, myocardial perfusion reserve index; PET, positron emission tomography; TTE, transthoracic echocardiography 


\section{DISCUSSION}

We provided an overview of currently used non-invasive imaging techniques and corresponding reference values for CMD in patients with AP and no or nonobstructive CAD as well as healthy subjects. We found quite some differences between reported non-invasive imaging parameters to assess CMD, which we have summarized in Figure 2. These differences reflect the large heterogeneity between the studied population as well as the rapidly developing imaging techniques and protocols per imaging technique, which result in a variety of different study protocols. Due to the heterogeneity between the included studies we were unable to perform a formal meta-analysis and provide clear clinically applicable cut-off values to diagnose CMD. MPRI was found to correlate well with invasive measurements obtained with CRT, such as index of microcirculatory resistance and CFR $(15,23)$. Therefore, MPRI could potentially serve as a non-invasive alternative to CRT. Current literature proposes two different cut-off values, namely 1.40 and $1.84(6,15)$, corresponding with the results found in this review. However, the results of this review suggest a grey area of MPRI values, as some overlap is seen between MPRI in patients and healthy controls. Stress $\mathrm{MBF}$ values can aid in differentiating CMD from normal coronary microvascular function. Liu et al. (15) have shown that a decreased stress MBF (i.e. less than 2.30 $\mathrm{ml} / \mathrm{min} / \mathrm{g}$ ) is suggestive of CMD in patients with inconclusive MPRI values. Furthermore, some CMR studies now investigate the clinical applicability of quantitative myocardial tissue characterization with rest and stress T1 mapping as an alternative $(29,33)$. Ischemic myocardial tissue can be differentiated from healthy tissue based on distinct properties at T1 mapping during rest and stress conditions, without the use of contrast agents. However, the diagnostic value of T1 mapping in diagnosing CMD still needs extensive validation (29).

Currently, PET is the most frequently applied and validated non-invasive imaging technique in quantifying microvascular blood flow. PET is considered the golden standard of non-invasive diagnosis of CMD, although discordance between invasive fractional flow reserve (FFR) and non-invasive CFR is reported in up to $30 \%$ of cases (16, $26,27,61)$. CMD is generally diagnosed with a CFR less than 2.0 if corrected for RPP or less than 2.5 if uncorrected $(28,35,45,47)$. However, no evidence-based cut-off values for CFR in PET are available yet. Similarly, no cut-off values for CFR in TTE have yet been determined and generally a cut-off value of less than 2.0 for the diagnosis of CMD is applied $(18,58,62-65)$. The study of Hildick-Smith et al. showed CFR with use of adenosine stress TTE to be well above this applied cut-off value, i.e. a mean CFR 3.7 in healthy controls and 5.9 in athletes (66). However these study population comprised men of 27 years of age and could therefore not be directly used as reference value for 
the, mainly older and female, population of interest at risk for CMD. TTE assessment of CFR with Doppler echocardiography has been validated against intracoronary Doppler measurements and outcomes correlate well $(2,5,60,67)$.

\section{Causes of heterogeneity in measured outcome parameters Patient groups}

The heterogeneity in outcomes observed in this systematic review is most likely the result of differences in inclusion criteria applied across several studies and differences in the use of imaging techniques. Although most studies investigated patients with typical AP and no or nonobstructive CAD during diagnostic CAG, the setting in which participants were recruited was not reported clearly. Furthermore, the definition of no or nonobstructive CAD and the control population was often unclear and, if documented, heterogeneous among the included studies (Additional file 6: Table S5). Therefore, a more homogeneous definition could not be applied in the search method. Hence, we suggest the use and documentation of standardized criteria as reported by COVADIS (12).

\section{Methodological differences}

Unclarity of the used reference standard, as reflected by the risk of bias assessment (Additional file 2: Table S1), may have introduced significant bias. Moreover, it was often unclear whether researchers were blinded to the reference standard when interpreting results from the index test.

Furthermore, measurement of MPRI in CMR might contribute to the inconsistent results observed in this systematic review. MPRI can be measured transmural, but also subendo- or epicardial. Several studies show subendocardial MPRI to be decreased more often than epicardial MPRI in CMD patients $(34,39,64,68)$, which might indicate subendocardial MPRI to be more valuable in diagnosing CMD as compared with epicardial or transmural MPRI. Unfortunately, in this systematic review only transmural MPRI values were included.

Regarding PET, correction for RPP is not standard which results in decreased comparability of outcomes. Moreover, the use of different radioactive tracers (150-water, $13 \mathrm{~N}$-ammonia and Rubidium-82) could result in varying outcomes due to differences in characteristics and processing of images $(14,27,28)$. The use of a specific radiotracer might require a specific cut-off value to diagnose CMD (14). Similar concerns apply to the use of various vasoactive agents to achieve hyperemia in stress perfusion imaging. Adenosine and dipyridamole are most commonly administered to achieve hyperemia. However, adenosine seems to be superior to dipyridamole 
with regard to attaining maximal hyperemia and their effects are not identical. Therefore the use of the different types and doses of vasoactive agent could have contributed to the differences found in the outcome parameters $(27,69,70)$.

Lastly, this systematic review highlights the discordance in nomenclature and reporting of outcomes. Standardization of outcome parameters reported could increase comparability of studies assessing reference values for the diagnosis of CMD.

\section{Sex differences}

In the present analysis women were highly represented in the patient groups (89\%) compared to control groups (33\%). Therefore, sex differences could contribute to discrepancies between studies resulting in decreased comparability between CFR and MPRI measurements in patient and control groups. Kobayashi et al. (17) measured coronary vascular diameter with quantitative CAG and intravascular ultrasound in patients with $A P$ and nonobstructive $C A D$ and found a smaller vascular diameter in women. Furthermore, they showed a significantly higher resting CBF in women. The latter is consistent with findings by Opstal et al. (71) and Chareonthaitawee et al. (72) who studied coronary blood flow in healthy subjects with $13 \mathrm{~N}$-ammonia PET ( $n=$ 206) and 150-water PET ( $n=169)$, respectively. These findings suggest sex differences in flow parameters. High resting myocardial flow volumes could decrease CFR (in PET and TTE) or MPRI (in CMR) in women compared to men as flow parameters are determined as the ratio of stress and rest perfusion. Although sex differences in resting MBF and CFR have been observed in invasive $\operatorname{CRT}(3,17,73)$, only one of the included studies included assessed sex differences regarding CBF and reported no significant sex differences (56). These findings are consistent with another study comparing non-invasive CFR between men and women using PET (74). Therefore, further research is needed to establish whether or not sex-specific cut-off values are required for the non-invasive diagnosis of CMD.

\section{Recommendations for future research}

The studies included in this review show heterogeneity in study methodology and outcome. This contributes to the discrepancies in outcomes between studies and to the lack of consensus regarding definition and cut-off values for CMD in non-invasive imaging modalities. We emphasize the need for large validation studies and suggest standardization of outcome parameters to reduce heterogeneity and increase comparability of studies. This is needed to provide clinically applicable, possibly sexspecific, reference values for the diagnosis of CMD in the future. 
Furthermore, during this systematic review, we found several other imaging modalities that are studied for their potential to diagnose CMD, such as myocardial contrast echocardiography (MCE) $(63,75,76)$, CT-perfusion (77) and absolute quantification of myocardial perfusion by CMR (78). Even though, current evidence is still limited so the clinical significance and applicability in regular care of these modalities in CMD diagnosis remains unclear, current research shows promising results. For example, Bechsgaard et al. studied CT myocardial perfusion in women with angina and no obstructive CAD, (defined as $<50 \%$ stenosis), in comparison with female controls (77). They showed CTperfusion is able to identify decreased global myocardial perfusion and impaired increase of myocardial blood flow during adenosine provocation in women with angina and non obstructive CAD as compared with the control group. The use of CT-perfusion in addition to the commonly performed CCTA could in the future play an important role in the evaluation of CMD early in the evaluation of patients with angina.

\section{Study limitations}

The number of studies investigating non-invasive imaging techniques to diagnose CMD is limited. As such, the results of this systematic review are based on limited data. Hence, only an indication of reference and cut-off values could be provided. Furthermore, a formal meta-analysis could not be performed due to heterogeneity of included studies. In addition, the risk of selection bias in the included studies was high. Also, the heterogeneity in the definition of non obstructive CAD might also have impacted the results as the included studies comprise patients with both completely normal or non obstructed coronaries. Unfortunately, as the definition of nonobstructive CAD was unclear or heterogeneous, it was not possible to separately analyze outcomes for patients with normal coronaries as compared to nonobstructive CAD. These analyses would have been of additive value as in patients with nonobstructive CAD there might still be epicardial stenosis that could impact myocardial blood flow. These limitations emphasize the importance of standardization of imaging protocols and analyses, patient selection and reporting of outcome measurements to obtain reliable and clinically relevant cut-off values for CMD.

\section{CONCLUSIONS}

This systematic review provided an overview of currently used parameters and cut-off values for CMD in patients with AP and no or nonobstructive CAD as well as healthy subjects. However, no definite cut-off values could be determined as no meta-analysis could be performed due to heterogeneity of studies investigating non-invasive imaging techniques in CMD. 


\section{Funding}

This study was funded by Dutch Heart Foundation (CVON 2017-22 ARGUS). The funding body played no role in the design of the study and collection, analysis, and interpretation of data and in writing the manuscript 


\section{REFERENCES}

1. Patel MR, Peterson ED, Dai D, Brennan JM, Redberg RF, Anderson HV, Brindis RG, Douglas PS (2010) Low diagnostic yield of elective coronary angiography. N Engl J Med 362:886-895 . https://doi.org/10.1056/NEJMoa0907272

2. Chen C, Wei J, AlBadri A, Zarrini P, Bairey Merz C (2016) Coronary Microvascular Dysfunction - Epidemiology, Pathogenesis, Prognosis, Diagnosis, Risk Factors and Therapy. Circ J 81:3-11 . https://doi.org/doi: 10.1253/circj. CJ-16-1002

3. Sara JD, Widmer RJ, Matsuzawa Y, Lennon RJ, Lerman LO, Lerman A (2015) Prevalence of Coronary Microvascular Dysfunction Among Patients With Chest Pain and Nonobstructive Coronary Artery Disease. JACC Cardiovasc Interv 8:1445-1453 . https://doi.org/10.1016/j.jcin.2015.06.017

4. Camici PG, Crea F (2007) Coronary Microvascular Dysfunction. N Engl J Med 356:830-40 . https://doi.org/10.1056/ NEJMra061889

5. Dean J, Cruz S Dela, Mehta PK, Merz CNB (2015) Coronary microvascular dysfunction: Sex-specific risk, diagnosis, and therapy. Nat Rev Cardiol 12:406-414 . https://doi.org/10.1038/nrcardio.2015.72

6. Thomson LEJ, Wei J, Agarwal M, Haft-Baradaran A, Shufelt C, Mehta PK, Gill EB, Johnson BD, Kenkre T, Handberg EM, Li D, Sharif B, Berman DS, Petersen JW, Pepine CJ, Merz CNB (2015) Cardiac magnetic resonance myocardial perfusion reserve index is reduced in women with coronary microvascular dysfunction: A national heart, lung, and blood institute-sponsored study from the women's ischemia syndrome evaluation. Circ Cardiovasc Imaging 8: . https://doi.org/10.1161/CIRCIMAGING.114.002481

7. Sharaf B, Wood T, Shaw L, Johnson BD, Kelsey S, Anderson RD, Pepine CJ, Bairey Merz CN (2013) Adverse outcomes among women presenting with signs and symptoms of ischemia and no obstructive coronary artery disease: Findings from the National Heart, Lung, and Blood Institute-sponsored Women's Ischemia Syndrome Evaluation (WISE) angiographic core lab. Am Heart J 166:134-141. https://doi.org/10.1016/j.ahj.2013.04.002

8. Taqueti VR, Shaw LJ, Cook NR, Murthy VL, Shah NR, Foster CR, Hainer J, Blankstein R, Dorbala S, Di Carli MF (2017) Excess Cardiovascular Risk in Women Relative to Men Referred for Coronary Angiography Is Associated with Severely Impaired Coronary Flow Reserve, Not Obstructive Disease. Circulation 135:566-577 . https://doi. org/10.1161/CIRCULATIONAHA.116.023266

9. Marinescu MA, Löffler Al, Ouellette M, Smith L, Kramer CM, Bourque JM (2015) Coronary microvascular dysfunction, microvascular angina, and treatment strategies. JACC Cardiovasc Imaging 8:210-220 . https://doi. org/10.1016/j.jcmg.2014.12.008

10. Murthy VL, Naya M, Taqueti VR, Foster CR, Gaber M, Hainer J, Dorbala S, Blankstein R, Rimoldi O, Camici PG, Di Carli MF (2014) Effects of sex on coronary microvascular dysfunction and cardiac outcomes. Circulation 129:2518-2527 . https://doi.org/10.1161/CIRCULATIONAHA.113.008507

11. Pepine CJ, Anderson RD, Sharaf BL, Reis SE, Smith KM, Handberg EM, Johnson BD, Sopko G, Bairey Merz CN (2010) Coronary Microvascular Reactivity to Adenosine Predicts Adverse Outcome in Women Evaluated for Suspected Ischemia. Results From the National Heart, Lung and Blood Institute WISE (Women's Ischemia Syndrome Evaluation) Study. J Am Coll Cardiol 55:2825-2832 . https://doi.org/10.1016/j.jacc.2010.01.054

12. Ong P, Camici PG, Beltrame JF, Crea F, Shimokawa H, Sechtem U, Kaski JC, Bairey Merz CN (2018) International standardization of diagnostic criteria for microvascular angina. Int J Cardiol 250:16-20 . https://doi.org/10.1016/j. ijcard.2017.08.068

13. Knuuti J, Wijns W, Achenbach S, Agewall S, Barbato E, Bax JJ, Capodanno D, Cuisset T, Deaton C, Dickstein K, Edvardsen T, Escaned J, Funck-Brentano C, Gersh BJ, Gilard M, Hasdai D, Hatala R, Mahfoud F, Masip J, Muneretto C, Prescott E, Saraste A, Storey RF, Svitil P, Valgimigli M, Windecker S, Aboyans V, Baigent C, Collet JP, Dean V, Delgado V, Fitzsimons D, Gale CP, Grobbee DE, Halvorsen S, Hindricks G, lung B, Jüni P, Katus HA, Landmesser U, Leclercq C, Lettino M, Lewis BS, Merkely B, Mueller C, Petersen S, Petronio AS, Richter DJ, Roffi M, Shlyakhto E, Simpson IA, Sousa-Uva M, Touyz RM, Benkhedda S, Metzler B, Sujayeva V, Cosyns B, Kusljugic Z, Velchev V, Panayi G, Kala P, Haahr-Pedersen SA, Kabil H, Ainla T, Kaukonen T, Cayla G, Pagava Z, Woehrle J, Kanakakis J, Toth K, Gudnason T, Peace A, Aronson D, Riccio C, Elezi S, Mirrakhimov E, Hansone S, Sarkis A, Babarskiene R, Beissel J, Cassar Maempel AJ, Revenco V, de Grooth GJ, Pejkov H, Juliebø V, Lipiec P, Santos J, Chioncel O, Duplyakov D, Bertelli L, Dikic AD, Studencan M, Bunc M, Alfonso F, Back M, Zellweger M, Addad F, Yildirir A, Sirenko Y, Clapp B (2020) 2019 ESC guidelines for the diagnosis and management of chronic coronary syndromes. Eur Heart J 41:407-477 . https:// doi.org/10.1093/eurheartj/ehz425 
14. Schindler TH, Dilsizian V (2020) Coronary Microvascular Dysfunction: Clinical Considerations and Noninvasive Diagnosis. JACC Cardiovasc Imaging 13:140-155 . https://doi.org/10.1016/j.jcmg.2018.11.036

15. Liu A, Wijesurendra RS, Liu JM, Forfar JC, Channon KM, Jerosch-Herold M, Piechnik SK, Neubauer S, Kharbanda RK, Ferreira VM (2018) Diagnosis of Microvascular Angina Using Cardiac Magnetic Resonance. J Am Coll Cardiol 71:969-979 . https://doi.org/10.1016/j.jacc.2017.12.046

16. Michelsen MM, Mygind ND, Pena A, Olsen RH, Christensen TE, Ghotbi AA, Hasbak P, Kjaer A, Gustafsson I, Hansen PR, Hansen HS, Høst N, Kastrup J, Prescott E (2017) Transthoracic Doppler echocardiography compared with positron emission tomography for assessment of coronary microvascular dysfunction: The iPOWER study. Int J Cardiol 228:435-443 . https://doi.org/10.1016/j.ijcard.2016.11.004

17. Kobayashi Y, Fearon WF, Honda Y, Tanaka S, Pargaonkar V, Fitzgerald PJ, Lee DP, Stefanick M, Yeung AC, Tremmel JA (2015) Effect of Sex Differences on Invasive Measures of Coronary Microvascular Dysfunction in Patients With Angina in the Absence of Obstructive Coronary Artery Disease. JACC Cardiovasc Interv 8:1433-1441 . https://doi. org/10.1016/j.jcin.2015.03.045

18. Nagel E, Klein C, Paetsch I, Hettwer S, Schnackenburg B, Wegscheider K, Fleck E (2003) Magnetic resonance perfusion measurements for the noninvasive detection of coronary artery disease. Circulation 108:432-437 . https:// doi.org/10.1161/01.CIR.0000080915.35024.A9

19. Anderson RD, Petersen JW, Mehta PK, Wei J, Johnson BD, Handberg EM, Kar S, Samuels B, Azarbal B, Kothawade K, Kelsey SF, Sharaf B, Shaw LJ, Sopko G, Bairey Merz CN, Pepine CJ (2019) Prevalence of Coronary Endothelial and Microvascular Dysfunction in Women with Symptoms of Ischemia and No Obstructive Coronary Artery Disease Is Confirmed by a New Cohort: The NHLBI-Sponsored Women's Ischemia Syndrome Evaluation-Coronary Vascular Dysfunc. J Interv Cardiol. https://doi.org/10.1155/2019/7169275

20. AlBadri A, Leong D, Bairey Merz CN, Wei J, Handberg EM, Shufelt CL, Mehta PK, Nelson MD, Thomson LE, Berman DS, Shaw LJ, Cook-Wiens G, Pepine CJ (2017) Typical angina is associated with greater coronary endothelia dysfunction but not abnormal vasodilatory reserve. Clin Cardiol 40:886-891 . https://doi.org/10.1002/clc.22740

21. Mathew RC, Bourque JM, Salerno M, Kramer CM (2020) Cardiovascular Imaging Techniques to Assess Microvascular Dysfunction. JACC Cardiovasc Imaging 13:1577-1590 . https://doi.org/10.1016/j.jcmg.2019.09.006

22. Whiting PF, Rutjes AWS, Westwood ME, Mallett S, Deeks JJ, Reitsma JB, Leeflang MMG, Sterne JAC, Bossuyt PMM (2011) Quadas-2: A revised tool for the quality assessment of diagnostic accuracy studies. Ann Intern Med 155:529-536 . https://doi.org/10.7326/0003-4819-155-8-201110180-00009

23. Wöhrle J, Nusser T, Merkle N, Kestler HA, Grebe OC, Marx N, Höher M, Kochs M, Hombach V (2006) Myocardial perfusion reserve in cardiovascular magnetic resonance: Correlation to coronary microvascular dysfunction. J Cardiovasc Magn Reson 8:781-787 . https://doi.org/10.1080/10976640600737649

24. Löffler Al, Bourque JM (2016) Coronary Microvascular Dysfunction, Microvascular Angina, and Management. Curr Cardiol Rep 18:1-7 . https://doi.org/10.1007/s11886-015-0682-9

25. Karamitsos TD, Arnold JR, Pegg TJ, Francis JM, Birks J, Jerosch-Herold M, Neubauer S, Selvanayagam JB (2012) Patients with syndrome $X$ have normal transmural myocardial perfusion and oxygenation: A 3-T cardiovascular magnetic resonance imaging study. Circ Cardiovasc Imaging 5:194-200 . https://doi.org/10.1161/CIRCIMAG ING.111.969667

26. Pelletier-Galarneau M, Martineau P, El Fakhri G (2019) Quantification of PET Myocardial Blood Flow. Curr Cardiol Rep 21: . https://doi.org/10.1007/s11886-019-1096-x

27. Feher A, Sinusas AJ (2017) Quantitative Assessment of Coronary Microvascular Function: Dynamic Single-Photon Emission Computed Tomography, Positron Emission Tomography, Ultrasound, Computed Tomography, and Magnetic Resonance Imaging. Circ Cardiovasc Imaging 10: . https://doi.org/10.1161/CIRCIMAGING.117.006427

28. Camici PG, D'Amati G, Rimoldi O (2015) Coronary microvascular dysfunction: Mechanisms and functional assessment. Nat Rev Cardiol 12:48-62 . https://doi.org/10.1038/nrcardio.2014.160 Introduction

29. Liu A, Wijesurendra RS, Liu JM, Greiser A, Jerosch-Herold M, Forfar JC, Channon KM, Piechnik SK, Neubauer S, Kharbanda RK, Ferreira VM (2018) Gadolinium-Free Cardiac MR Stress T1-Mapping to Distinguish Epicardial From Microvascular Coronary Disease. J Am Coll Cardiol 71:957-968 . https://doi.org/10.1016/j.jacc.2017.11.071

30. Rahman H, Ryan M, Lumley M, Modi B, Mcconkey H, Ellis H, Scannell C, Clapp B, Marber M, Webb A, Chiribiri A, Perera D (2019) Coronary Microvascular Dysfunction Is Associated With Myocardial Ischemia and Abnormal Coronary Perfusion During Exercise. Circulation 140:1805-1816 . https://doi.org/10.1161/CIRCULATIONAHA.119.041595

31. Jaarsma C, Vink H, van Haare J, Bekkers SCAM, van Rooijen BD, Backes WH, Wildberger JE, Crijns HJ, van Teeffelen J, Schalla S (2017) Non-invasive assessment of microvascular dysfunction in patients with microvascular angina. Int J Cardiol 248:433-439 . https://doi.org/10.1016/j.ijcard.2017.05.010 
32. Mehta PK, Goykhman P, Thomson LEJ, Shufelt C, Wei J, Yang Y, Gill E, Minissian M, Shaw LJ, Slomka PJ, Slivka M, Berman DS, Bairey Merz CN (2011) Ranolazine improves angina in women with evidence of myocardial ischemia but no obstructive coronary artery disease. JACC Cardiovasc Imaging 4:514-522 . https://doi.org/10.1016/j. jcmg.2011.03.007

33. Zorach B, Shaw PW, Bourque J, Kuruvilla S, Balfour PC, Yang Y, Mathew R, Pan J, Gonzalez JA, Taylor AM, Meyer $\mathrm{CH}$, Epstein FH, Kramer CM, Salerno M (2018) Quantitative cardiovascular magnetic resonance perfusion imaging identifies reduced flow reserve in microvascular coronary artery disease. J Cardiovasc Magn Reson 20: . https:// doi.org/10.1186/s12968-018-0435-1

34. Panting JR, Gatehouse PD, Yang GZ, Grothues F, Firmin DN, Collins P, Pennell DJ (2002) Abnormal subendocardial perfusion in cardiac syndrome $X$ detected by cardiovascular magnetic resonance imaging. N Engl J Med 346:19481953. https://doi.org/10.1056/NEJMoa012369

35. Pärkkä JP, Niemi P, Saraste A, Koskenvuo JW, Komu M, Oikonen V, Toikka JO, Kiviniemi TO, Knuuti J, Sakuma H, Hartiala JJ (2006) Comparison of MRI and positron emission tomography for measuring myocardial perfusion reserve in healthy humans. Magn Reson Med 55:772-779. https://doi.org/10.1002/mrm.20833

36. Vermeltfoort I, Bondarenko O, Raijmakers P, Odekerken D, Kuijper A, Zwijnenburg A, Van der Vis-Melsen M, Twisk J, Beek M, Teule G, Van Rossum A (2007) Is subendocardial ischaemia present in patients with chest pain and normal coronary angiograms? A cardiovascular MR study (5). Eur Heart J 28:2687-2688 . https://doi.org/10.1093/ eurheartj/ehm381

37. Nelson MD, Szczepaniak LS, Wei J, Haftabaradaren A, Bharadwaj M, Sharif B, Mehta P, Zhang X, Thomson LE, Berman DS, Li D, Merz CNB (2014) Diastolic dysfunction in women with signs and symptoms of ischemia in the absence of obstructive coronary artery disease: A hypothesis-generating study. Circ Cardiovasc Imaging 7:510-516 . https://doi.org/10.1161/CIRCIMAGING.114.001714

38. Merz CNB, Handberg EM, Shufelt CL, Mehta PK, Minissian MB, Wei J, Thomson LEJ, Berman DS, Shaw LJ, Petersen JW, Brown GH, Anderson RD, Shuster JJ, Cook-Wiens G, Rogatko A, Pepine CJ (2016) A randomized, placebocontrolled trial of late $\mathrm{Na}$ current inhibition (ranolazine) in coronary microvascular dysfunction (CMD): Impact on angina and myocardial perfusion reserve. Eur Heart J 37:1504-1513 . https://doi.org/10.1093/eurheartj/ehv647

39. Bakir M, Wei J, Nelson MD, Mehta PK, Haftbaradaran A, Jones E, Gill E, Sharif B, Slomka PJ, Li D, Shufelt CL, Minissian M, Berman DS, Noel Bairey Merz C, Thomson LEJ (2016) Cardiac magnetic resonance imaging for myocardial perfusion and diastolic function-reference control values for women. Cardiovasc Diagn Ther 6:78-86 . https://doi. org/10.3978/j.issn.2223-3652.2015.09.03

40. Uusitalo V, Saraste A, Kajander S, Luotolahti M, Wendelin-Saarenhovi M, Sundell J, Raitakari O, Knuuti J (2013) The association between coronary flow reserve and development of coronary calcifications: a follow-up study for 11 years in healthy young men. Eur Heart J Cardiovasc Imaging 14:812-818 . https://doi.org/10.1093/ehjci/jes301

41. Buus NH, Bøttcher M, Bøtker HE, Sørensen KE, Nielsen TT, Mulvany MJ (1999) Reduced vasodilator capacity in syndrome $X$ related to structure and function of resistance arteries. Am J Cardiol. https://doi.org/10.1016/S00029149(98)00815-7

42. Bøttcher M, Bøtker HE, Sonne H, Nielsen TT, Czernin J (1999) Endothelium-dependent and -independent perfusion reserve and the effect of L-arginine on myocardial perfusion in patients with syndrome X. Circulation 99:1795-1801 . https://doi.org/10.1161/01.CIR.99.14.1795

43. Meeder JG, Blanksma PK, Van Der Wall EE, Willemsen ATM, Pruim J, Anthonio RL, De Jong RM, Vaalburg W, Lie KI (1997) Coronary vasomotion in patients with syndrome X: Evaluation with positron emission tomography and parametric myocardial perfusion imaging. Eur J Nucl Med 24:530-537. https://doi.org/10.1007/BF01267685

44. Marroquin OC, Holubkov R, Edmundowicz D, Rickens C, Pohost G, Buchthal S, Pepine CJ, Sopko G, Sembrat RC, Meltzer CC, Reis SE (2003) Heterogeneity of microvascular dysfunction in women with chest pain not attributable to coronary artery disease: Implications for clinical practice. Am Heart J 145:628-635 . https://doi.org/10.1067/ mhj.2003.95

45. Graf S, Khorsand A, Gwechenberger M, Schütz M, Kletter K, Sochor H, Dudczak R, Maurer G, Pirich C, Porenta G, Zehetgruber $M(2006)$ Myocardial perfusion in patients with typical chest pain and normal angiogram. Eur J Clin Invest 36:326-332 . https://doi.org/10.1111/j.1365-2362.2006.01635.x

46. De Vries J, Dejongste MJL, Jessurun GAJ, Jager PL, Staal MJ, Slart RHJA, Slart HJA (2006) Myocardial perfusion quantification in patients suspected of cardiac syndrome $X$ with positive and negative exercise testing: $A(13 \mathrm{~N})$ ammonia positron emission tomography study. Lippincott Williams \& Wilkins

47. Graf S, Khorsand A, Gwechenberger M, Novotny C, Kletter K, Sochor H, Pirich C, Maurer G, Porenta G, Zehetgruber $M$ (2007) Typical chest pain and normal coronary angiogram: Cardiac risk factor analysis versus PET for detection of microvascular disease. J Nucl Med 48:175-181 
48. Scholtens AM, Tio RA, Willemsen A, Dierckx RAJO, Boersma HH, Zeebregts CJ, Glaudemans AWJM, Slart RHJA (2011) Myocardial perfusion reserve compared with peripheral perfusion reserve: A (13N)ammonia PET study. J Nucl Cardiol 18:238-246. https://doi.org/10.1007/s12350-011-9339-2

49. Vaccarino V, Khan D, Votaw J, Faber T, Veledar E, Jones DP, Goldberg J, Raggi P, Quyyumi AA, Bremner JD (2011) Inflammation is related to coronary flow reserve detected by positron emission tomography in asymptomatic male twins. J Am Coll Cardiol 57:1271-1279 . https://doi.org/10.1016/j.jacc.2010.09.074

50. Vermeltfoort IA, Raijmakers PG, Lubberink M, Germans T, Van Rossum AC, Lammertsma AA, Knaapen P (2011) Feasibility of subendocardial and subepicardial myocardial perfusion measurements in healthy normals with 150-labeled water and positron emission tomography. J Nucl Cardiol 18:650-656 . https://doi.org/10.1007/ s12350-011-9375-y

51. Galiuto L, Sestito A, Barchetta S, Sgueglia GA, Infusino F, La Rosa C, Lanza G, Crea F (2007) Noninvasive Evaluation of Flow Reserve in the Left Anterior Descending Coronary Artery in Patients With Cardiac Syndrome X. Am J Cardiol 99:1378-1383 . https://doi.org/10.1016/j.amjcard.2006.12.070

52. Mygind ND, Michelsen MM, Pena A, Qayyum AA, Frestad D, Christensen TE, Ghotbi AA, Dose N, Faber R, Vejlstrup N, Hasbak P, Kjaer A, Prescott E, Kastrup J (2016) Coronary microvascular function and myocardial fibrosis in women with angina pectoris and no obstructive coronary artery disease: the iPOWER study. J Cardiovasc Magn Reson 18:1-12 . https://doi.org/10.1186/s12968-016-0295-5

53. Cemin R, Erlicher A, Fattor B, Pitscheider W, Cevese A (2008) Reduced coronary flow reserve and parasympathetic dysfunction in patients with cardiovascular syndrome X. Coron Artery Dis 19:1-7 . https://doi.org/10.1097/ MCA.0b013e3282f18e8d

54. Lanza GA, Buffon A, Sestito A, Natale L, Sgueglia GA, Galiuto L, Infusino F, Mariani L, Centola A, Crea F (2008) Relation Between Stress-Induced Myocardial Perfusion Defects on Cardiovascular Magnetic Resonance and Coronary Microvascular Dysfunction in Patients With Cardiac Syndrome X. J Am Coll Cardiol 51:466-472 . https://doi. org/10.1016/j.jacc.2007.08.060

55. Monaco A Di, Bruno I, Sestito A, Lamendola P, Barone L, Bagnato A, Nerla R, Pisanello C, Giordano A, Lanza GA, Crea $F$ (2009) Cardiac adrenergic nerve function and microvascular dysfunction in patients with cardiac syndrome X. Heart 95:550-554 . https://doi.org/10.1136/hrt.2008.156851

56. Sestito A, Lanza GA, Di Monaco A, Lamendola P, Careri G, Tarzia P, Pinnacchio G, Battipaglia I, Crea F (2011) Relation between cardiovascular risk factors and coronary microvascular dysfunction in cardiac syndrome X. J Cardiovasc Med 12:322-327 . https://doi.org/10.2459/JCM.0b013e3283406479

57. Di Franco A, Lanza GA, Di Monaco A, Sestito A, Lamendola P, Nerla R, Tarzia P, Virdis D, Vollono C, Valeriani M, Crea $F$ (2012) Coronary microvascular function and cortical pain processing in patients with silent positive exercise testing and normal coronary arteries. Am J Cardiol 109:1705-1710 . https://doi.org/10.1016/j.amjcard.2012.02.012

58. Tagliamonte E, Rigo F, Cirillo T, Astarita C, Quaranta G, Marinelli U, Caruso A, Romano C, Capuano N (2015) Effects of ranolazine on noninvasive coronary flow reserve in patients with myocardial ischemia but without obstructive coronary artery disease. Echocardiography 32:516-521. https://doi.org/10.1111/echo.12674

59. Wu M, Villano A, Russo G, Di Franco A, Stazi A, Lauria C, Sestito A, Lanza GA, Crea F (2015) Poor tolerance and limited effects of isosorbide-5- mononitrate in microvascular angina. Cardiol 130:201-206 . https://doi. org/10.1159/000370027

60. Anchisi C, Marti G, Bellacosa I, Mary D, Vacca G, Marino P, Grossini E (2017) Coronary flow reserve/diastolic function relationship in angina-suffering patients with normal coronary angiography. J Cardiovasc Med 18:325-331 https://doi.org/10.2459/JCM.0000000000000344

61. Slomka P, Berman DS, Alexanderson E, Germano G (2014) The role of PET quantification in cardiovascular imaging. Clin Transl Imaging 2:343-358 . https://doi.org/10.1007/s40336-014-0070-2

62. Kuruvilla S, Kramer CM (2013) Coronary microvascular dysfunction in women: An overview of diagnostic strategies. Expert Rev Cardiovasc Ther 11:1515-1525 . https://doi.org/10.1586/14779072.2013.833854

63. Nel K, Nam MCY, Anstey C, Boos CJ, Carlton E, Senior R, Kaski JC, Khattab A, Shamley D, Byrne CD, Stanton T, Greaves K (2017) Myocardial blood flow reserve is impaired in patients with aortic valve calcification and unobstructed epicardial coronary arteries. Int J Cardiol 248:427-432 . https://doi.org/10.1016/j.ijcard.2017.06.023

64. Kato S, Saito N, Kirigaya H, Gyotoku D, linuma N, Kusakawa Y, Iguchi K, Nakachi T, Fukui K, Futaki M, Iwasawa T, Kimura K, Umemura S (2016) Impairment of coronary flow reserve evaluated by phase contrast cine-magnetic resonance imaging in patients with heart failure with preserved ejection fraction. J Am Heart Assoc 5:1-9. https:// doi.org/10.1161/JAHA.115.002649

65. Sade LE, Eroglu S, Bozbaş H, Özbiçer S, Hayran M, Haberal A, Müderriso?llu H (2009) Relation between epicardial fat thickness and coronary flow reserve in women with chest pain and angiographically normal coronary arteries. 
Atherosclerosis 204:580-585 . https://doi.org/10.1016/j.atherosclerosis.2008.09.038

66. Hildick-Smith DJR, Johnson PJ, Wisbey CR, Winter EM, Shapiro LM (2000) Coronary flow reserve is supranormal in endurance athletes: An adenosine transthoracic echocardiographic study. Heart 84:383-389 . https://doi. org/10.1136/heart.84.4.383

67. Hozumi T, Yoshida K, Akasaka T, Asami Y, Ogata Y, Takagi T, Kaji S, Kawamoto T, Ueda Y, Morioka S (1998) Noninvasive assessment of coronary flow velocity and coronary flow velocity reserve in the left anterior descending coronary artery by Doppler echocardiography: Comparison with invasive technique. J Am Coll Cardiol 32:1251-1259 . https://doi.org/10.1016/S0735-1097(98)00389-1

68. Shufelt CL, Thomson LEJ, Goykhman P, Agarwal M, Mehta PK, Sedlak T, Li N, Gill E, Samuels B, Azabal B, Kar S, Kothawade K, Minissian M, Slomka P, Berman DS, Bairey Merz CN (2013) Cardiac magnetic resonance imaging myocardial perfusion reserve index assessment in women with microvascular coronary dysfunction and reference controls. Cardiovasc Diagn Ther 3:153-15360 . https://doi.org/10.3978/j.issn.2223-3652.2013.08.02

69. Vasu S, Bandettini WP, Hsu LY, Kellman P, Leung S, Mancini C, Shanbhag SM, Wilson J, Booker OJ, Arai AE (2013) Regadenoson and adenosine are equivalent vasodilators and are superior than dipyridamole- a study of first pass quantitative perfusion cardiovascular magnetic resonance. J Cardiovasc Magn Reson 15: . https://doi. org/10.1186/1532-429X-15-85

70. Lim HE, Shim WJ, Rhee H, Kim SM, Hwang GS, Kim YH, Seo HS, Oh DJ, Ro YM (2000) Assessment of coronary flow reserve with transthoracic Doppler echocardiography: Comparison among adenosine, standard-dose dipyridamole, and high-dose dipyridamole. J Am Soc Echocardiogr 13:264-270 . https://doi.org/10.1067/mje.2000.103508

71. Opstal T, Knol R, Cornel J, Wondergem M, Van der Zant F (2018) Myocardial blood flow and myocardial flow reserve values in $13 \mathrm{~N}$-ammonia myocardial perfusion $\mathrm{PET} / \mathrm{CT}$ using a time-efficient protocol in patients without coronary artery disease. Eur J Hybrid Imaging 2: . https://doi.org/10.1186/s41824-018-0029-z

72. Chareonthaitawee P, Kaufmann PA, Rimoldi O, Camici PG (2001) Heterogeneity of resting and hyperemic myocardial blood flow in healthy humans. Cardiovasc Res 50:151-161 . https://doi.org/10.1016/S0008-6363(01)00202-4

73. Waheed N, Elias-Smale S, Malas W, Maas AH, Sedlak TL, Tremmel J, Mehta PK (2020) Sex differences in nonobstructive coronary artery disease. Cardiovasc Res 116:829-840 . https://doi.org/10.1093/cvr/cvaa001

74. Safdar B, D'Onofrio G, Dziura J, Russell RR, Johnson C, Sinusas AJ (2020) Prevalence and characteristics of coronary microvascular dysfunction among chest pain patients in the emergency department. Eur Hear J Acute Cardiovasc Care 9:5-13 . https://doi.org/10.1177/2048872618764418

75. Taqui S, Ferencik M, Davidson BP, Belcik JT, Moccetti F, Layoun M, Raber J, Turker M, Tavori H, Fazio S, Lindner JR (2019) Coronary Microvascular Dysfunction by Myocardial Contrast Echocardiography in Nonelderly Patients Referred for Computed Tomographic Coronary Angiography. J Am Soc Echocardiogr 32:817-825 . https://doi. org/10.1016/j.echo.2019.03.001

76. Rinkevich D, Belcik T, Gupta NC, Cannard E, Alkayed NJ, Kaul S (2013) Coronary autoregulation is abnormal in syndrome X: Insights using myocardial contrast echocardiography. J Am Soc Echocardiogr 26:290-296 . https://doi. org/10.1016/j.echo.2012.12.008

77. Bechsgaard DF, Gustafsson I, Michelsen MM, Mygind ND, Raft KF, Linde JJ, Kofoed KF, Lin FYH, Min JK, Prescott E, Hove JD (2020) Evaluation of computed tomography myocardial perfusion in women with angina and no obstructive coronary artery disease. Int J Cardiovasc Imaging 36:367-382 . https://doi.org/10.1007/s10554-019-01723-5

78. Engblom H, Xue H, Akil S, Carlsson M, Hindorf C, Oddstig J, Hedeer F, Hansen MS, Aletras AH, Kellman P, Arheden H (2017) Fully quantitative cardiovascular magnetic resonance myocardial perfusion ready for clinical use: $A$ comparison between cardiovascular magnetic resonance imaging and positron emission tomography. J Cardiovasc Magn Reson 19: . https://doi.org/10.1186/s12968-017-0388-9 
Table 1. Characteristics of the included studies

\begin{tabular}{|c|c|c|c|c|c|}
\hline $\begin{array}{l}\text { Author } \\
\text { (year) }\end{array}$ & Study design & Patient group & $\mathbf{N}=$ & $\begin{array}{l}\text { Mean age } \\
( \pm S D)\end{array}$ & $\begin{array}{l}\text { Sex } \\
\text { (F/M) }\end{array}$ \\
\hline $\begin{array}{l}\text { Meeder } \\
(1997)\end{array}$ & Case-control & $\begin{array}{l}\text { Patients with syndrome } X \text { with typical cardiac } \\
\text { chest pain with exercise-induced ischemic- } \\
\text { appearing electrocardiographic changes (>1 } \\
\text { mm horizontal ST-T segment depression) } \\
\text { and/or reversible myocardial perfusion } \\
\text { defects at thallium-201 perfusion scintigraphy } \\
\text { and no significant CAD on CAG. Gastro- } \\
\text { intestinal causes of chest pain were excluded. }\end{array}$ & 25 & $51 \pm 9$ & $16 / 9$ \\
\hline $\begin{array}{l}\text { Bottcher } \\
\text { (1999) }\end{array}$ & Case-control & $\begin{array}{l}\text { Angina pectoris and positive stress ECG, } \\
\text { normal CAG without risk factors for CAD. }\end{array}$ & 25 & $53 \pm 7$ & $25 / 0$ \\
\hline $\begin{array}{l}\text { Buus } \\
(1999)\end{array}$ & Case-control & $\begin{array}{l}\text { Typical effort angina, positive stress ECG, } \\
\text { normal CAG and TTE. No history of } \\
\text { hypertension or diabetes mellitus. }\end{array}$ & 16 & $56.6 \pm 1.2$ & $13 / 3$ \\
\hline $\begin{array}{l}\text { Panting } \\
(2002)\end{array}$ & Case-control & $\begin{array}{l}\text { Typical effort angina, abnormal stress ECG, } \\
\text { normal CAG recruited from Women's Heart } \\
\text { Disease Clinic at Royal Brompton Hospital } \\
\text { (London). }\end{array}$ & 20 & $55.9 \pm 10.5$ & $16 / 4$ \\
\hline $\begin{array}{l}\text { Marroquin } \\
(2003)\end{array}$ & Case-control & $\begin{array}{l}\text { Women with chest pain and epicardial } \\
\text { coronaries that were angiographically normal } \\
\text { or with only minimal luminal irregularities } \\
\text { ( }<50 \% \text { stenoses) who were enrolled in the } \\
\text { WISE study at the University of Pittsburgh. }\end{array}$ & 34 & $52.1 \pm 10.0$ & $34 / 0$ \\
\hline $\begin{array}{l}\text { De Vries } \\
(2006)\end{array}$ & Case-control & $\begin{array}{l}\text { Typical chest pain and normal CAG. Exclusion: } \\
\text { LBBB on ECG, first degree AV block and } \\
\text { diabetes mellitus. }\end{array}$ & 42 & $58 \pm 12$ & $26 / 16$ \\
\hline $\begin{array}{l}\text { Graf } \\
(2006)\end{array}$ & Case-control & $\begin{array}{l}\text { Typical angina, normal CAG and positive } \\
\text { stress ECG or SPECT, exclusion of myocardial } \\
\text { or valvular disease by TTE. Exclusion: diabetes } \\
\text { mellitus. }\end{array}$ & 58 & $58 \pm 10$ & $39 / 19$ \\
\hline $\begin{array}{l}\text { Pärkkä } \\
(2006)\end{array}$ & $\begin{array}{l}\text { Cross- } \\
\text { sectional/ } \\
\text { descriptive }\end{array}$ & $\mathrm{N} / \mathrm{A}$ & $\mathrm{N} / \mathrm{A}$ & $\mathrm{N} / \mathrm{A}$ & $\mathrm{N} / \mathrm{A}$ \\
\hline $\begin{array}{l}\text { Wöhrle } \\
\text { (2006) }\end{array}$ & Case series & Typical angina pectoris and normal CAG & 12 & $61.8 \pm 8.2$ & $7 / 5$ \\
\hline $\begin{array}{l}\text { Galiuto } \\
\text { (2007) }\end{array}$ & Case-control & $\begin{array}{l}\text { Typical effort angina, positive stress ECG and } \\
\text { normal CAG. Exclusion: moderate to severe } \\
\text { hypertension, diabetes mellitus, other heart } \\
\text { disease or contraindications to adenosine } \\
\text { infusion. }\end{array}$ & 17 & $55 \pm 10$ & $9 / 8$ \\
\hline $\begin{array}{l}\text { Graf } \\
(2007)\end{array}$ & Case-control & $\begin{array}{l}\text { Typical angina, normal CAG and positive } \\
\text { stress ECG or SPECT. Myocardial or valve } \\
\text { disease excluded by TTE. Exclusion: diabetes } \\
\text { mellitus and other major diseases. }\end{array}$ & 79 & $58 \pm 10$ & $52 / 27$ \\
\hline
\end{tabular}




\begin{tabular}{lcllll} 
Control group & $\mathbf{N}=$ & $\begin{array}{l}\text { Mean age } \\
\text { ( } \pm \text { SD) }\end{array}$ & $\begin{array}{l}\text { Sex } \\
\text { (F/M) }\end{array}$ & $\begin{array}{l}\text { Imaging modality } \\
\text { (outcome } \\
\text { parameter) }\end{array}$ & $\begin{array}{l}\text { Vasoactive agent } \\
\text { used (dose) }\end{array}$ \\
\hline Healthy volunteers & 21 & $42 \pm 13$ & $8 / 13$ & $\begin{array}{l}\text { PET (MPR), N-13 } \\
\text { ammonia }\end{array}$ & $\begin{array}{l}\text { dipyridamole } \\
(0.56 \mathrm{mg} / \mathrm{kg} \text { per } 4 \\
\text { minutes) }\end{array}$
\end{tabular}

$\begin{array}{lcccll}\begin{array}{l}\text { Healthy age and sex matched } \\ \text { volunteers }\end{array} & 15 & 54 \pm 10 & 15 / 0 & \begin{array}{l}\text { PET (CFR), N-13 } \\ \text { ammonia }\end{array} & \begin{array}{l}\text { dipyridamole } \\ \text { (0.56 mg/kg per } 4 \\ \text { minutes) }\end{array} \\ \begin{array}{l}\text { Healthy subjects (recruited among } \\ \text { blood donors and hospital staff) }\end{array} & 15 & 53.5 \pm 1.1 & 12 / 3 & \begin{array}{l}\text { PET (CFR), N-13 } \\ \text { ammonia }\end{array} & \begin{array}{l}\text { dipyridamole } \\ \text { (0.56 mg/kg per } 4 \\ \text { minutes) }\end{array} \\ \begin{array}{l}\text { Healthy age and sex matched } \\ \text { subjects, no history of chest pain } \\ \text { and low cardiovascular risk profile. }\end{array} & 10 & 57.9 \pm 7.4 & 8 / 2 & \text { CMR (MPRI), 1.5T } & \begin{array}{l}\text { adenosine } \\ \text { (140 mcg } / \mathrm{kg} / \mathrm{min}\end{array} \\ \begin{array}{l}\text { No SPECT or CAG was performed. } \\ \text { fealthy age-matched women. }\end{array} & 9 & 50.4 \pm 12.2 & 9 / 0 & \begin{array}{l}\text { PET (CFR), 13-N } \\ \text { ammonia }\end{array} & \begin{array}{l}\text { adenosine } \\ \text { (140 mcg } / \mathrm{kg} / \mathrm{min} \\ \text { for } 4 \mathrm{minutes})\end{array}\end{array}$

\begin{tabular}{|c|c|c|c|c|c|}
\hline $\begin{array}{l}\text { Healthy volunteers without chest } \\
\text { pain or CAD. }\end{array}$ & 21 & N/A & N/A & $\begin{array}{l}\text { PET (CFR), N-13 } \\
\text { ammonia }\end{array}$ & $\begin{array}{l}\text { dipyridamole } \\
(0.56 \mathrm{mg} / \mathrm{kg} \text { per } 6 \\
\mathrm{min})\end{array}$ \\
\hline N/A & $\mathrm{N} / \mathrm{A}$ & N/A & $\mathrm{N} / \mathrm{A}$ & $\begin{array}{l}\text { PET (CFR), N-13 } \\
\text { ammonia }\end{array}$ & $\begin{array}{l}\text { dipyridamole } \\
(0.56 \mathrm{mg} / \mathrm{kg} \text { per } 4 \\
\mathrm{min})\end{array}$ \\
\hline
\end{tabular}

\begin{tabular}{|c|c|c|c|c|c|}
\hline $\begin{array}{l}\text { Male volunteers, nonsmoking. One } \\
\text { patient with hypertension, others } \\
\text { no history of cardiovascular disease. }\end{array}$ & 18 & $40.0 \pm 14.4$ & $0 / 18$ & $\begin{array}{l}\text { CMR (MPR), 1.5T } \\
\text { PET (MPR), } \\
\text { 150-labeled water }\end{array}$ & $\begin{array}{l}\text { dipyridamole } \\
(0.56 \mathrm{mg} / \mathrm{kg} \text { per } 4 \\
\mathrm{min})\end{array}$ \\
\hline N/A & $\mathrm{N} / \mathrm{A}$ & N/A & N/A & CMR (MPRI), 1.5T & $\begin{array}{l}\text { adenosine } \\
(140 \mathrm{mcg} / \mathrm{min} / \mathrm{kg} \\
\text { for } 3 \mathrm{~min})\end{array}$ \\
\hline $\begin{array}{l}\text { Healthy subjects age and sex } \\
\text { matched. Exclusion: moderate to } \\
\text { severe hypertension, diabetes } \\
\text { mellitus, other heart disease or }\end{array}$ & 17 & $55 \pm 10$ & $10 / 7$ & $\begin{array}{l}\text { TTE (CFR), distal } \\
\text { LAD with } \\
\text { pulse-wave } \\
\text { Doppler }\end{array}$ & $\begin{array}{l}\text { adenosine } \\
\text { (140 } \mathrm{mcg} / \mathrm{kg} \text { in } 90 \\
\text { seconds) }\end{array}$ \\
\hline
\end{tabular}

se or infusion.

$\begin{array}{lllll}\begin{array}{l}\text { Atypical chest pain, normal CAG and } \\ \text { negative stress test. Myocardial or }\end{array} & 53 \pm 11 & 6 / 4 & \begin{array}{l}\text { PET (CFR), N-13 } \\ \text { ammonia }\end{array} & \begin{array}{l}\text { dipyridamole } \\ \text { (0.56 mg/kg per } 4 \\ \text { valve disease excluded by TTE. }\end{array} \\ \begin{array}{l}\text { Exclusion: diabetes mellitus and } \\ \text { other major diseases. }\end{array} & & & \text { min) }\end{array}$


Table 1. Continued

\begin{tabular}{|c|c|c|c|c|c|}
\hline $\begin{array}{l}\text { Author } \\
\text { (year) }\end{array}$ & Study design & Patient group & $\mathbf{N}=$ & $\begin{array}{l}\text { Mean age } \\
( \pm S D)\end{array}$ & $\begin{array}{l}\text { Sex } \\
\text { (F/M) }\end{array}$ \\
\hline $\begin{array}{l}\text { Vermeltfoort } \\
(2007)\end{array}$ & Case series & $\begin{array}{l}\text { Effort angina, positive stress ECG or SPECT } \\
\text { and normal CAG. Exclusion: history of heart } \\
\text { disease, hypertension, diabetes mellitus, } \\
\text { absence of pain without medication, } \\
\text { contra-indication for CMR. }\end{array}$ & 20 & $55 \pm 11$ & $15 / 5$ \\
\hline $\begin{array}{l}\text { Cemin } \\
(2008)\end{array}$ & Case-control & $\mathrm{N} / \mathrm{A}$ & $\mathrm{N} / \mathrm{A}$ & $\mathrm{N} / \mathrm{A}$ & N/A \\
\hline
\end{tabular}

\begin{tabular}{llllll}
\hline Lanza & Case-control & Effort angina, positive stress test and normal & 18 & $58 \pm 7$ & $11 / 7$ \\
(2008) & CAG. Exclusion: history of heart disease or & & & \\
& systemic diseases.
\end{tabular}

\begin{tabular}{|c|c|c|c|c|c|}
\hline $\begin{array}{l}\text { Di Monaco } \\
\text { (2009) }\end{array}$ & Case-control & $\begin{array}{l}\text { Patients presenting with effort angina, } \\
\text { positive stress test and normal CAG in a } \\
\text { university hospital. Exclusion: previous } \\
\text { enrollment in SPECT study. }\end{array}$ & 29 & $59 \pm 7$ & $18 / 11$ \\
\hline $\begin{array}{l}\text { Mehta } \\
\text { (2011) }\end{array}$ & RCT & $\begin{array}{l}\text { Women with chest pain and abnormal stress } \\
\text { testing, no obstructive CAD }(<50 \%) \text { on CAG. } \\
\text { Exclusion: renal failure or hepatic } \\
\text { insufficiency, contraindication to withholding } \\
\text { nitrates, calcium channel agents and } \\
\text { beta-adrenergic blockers for } 24 \mathrm{~h} \text {, } \\
\text { contraindication to CMR and use of drugs } \\
\text { inhibiting CYP3A. }\end{array}$ & 20 & $57 \pm 11$ & $20 / 0$ \\
\hline $\begin{array}{l}\text { Scholtens } \\
\text { (2011) }\end{array}$ & Case-control & $\begin{array}{l}\text { Patients submitted for PET analysis because } \\
\text { of typical chest pain, positive stress ECG and } \\
\text { normal CAG. }\end{array}$ & 14 & $\begin{array}{l}55(34-76) \\
\text { Median } \\
\text { (range) }\end{array}$ & $10 / 4$ \\
\hline $\begin{array}{l}\text { Sestito } \\
\text { (2011) }\end{array}$ & Case-control & $\begin{array}{l}\text { Patients with a history of effort angina, } \\
\text { positive stress test and normal CAG } \\
\text { undergoing clinical follow-up. Exclusion: } \\
\text { other cardiac or systemic disease. }\end{array}$ & 71 & $56 \pm 9$ & $48 / 23$ \\
\hline Vaccarino & Cohort & N/A & $\mathrm{N} / \mathrm{A}$ & N/A & $\mathrm{N} / \mathrm{A}$ \\
\hline
\end{tabular}

(2011)

\begin{tabular}{llllll}
\hline $\begin{array}{l}\text { Vermeltfoort } \\
(2011)\end{array}$ & Case series & N/A & N/A & N/A & N/A \\
\hline $\begin{array}{l}\text { Di Franco } \\
(2012)\end{array}$ & Case-control & $\begin{array}{l}\text { Effort angina, positive stress test and normal } \\
\text { CAG enrolled at outpatient ambulatory clinic. }\end{array}$ & & $61 \pm 5$ & $9 / 5$ \\
\end{tabular}

\begin{tabular}{lllll}
\hline $\begin{array}{l}\text { Karamitsos } \\
\text { (2012) }\end{array}$ & Case-control & $\begin{array}{l}\text { Typical effort angina, abnormal stress ECG } \\
\text { and normal CAG. Exclusion: diabetes mellitus, } \\
\text { hypertension and other cardiac or systemic } \\
\text { disease. }\end{array}$ \\
\hline
\end{tabular}




\section{Control group}

N/A
$\mathbf{N}=\quad$ Mean age $( \pm \mathrm{SD})$

\section{Sex Imaging modality \\ (F/M) (outcome parameter)}

N/A N/A
N/A CMR (MPRI), 1.5T adenosine

Vasoactive agent used (dose) (140 $\mathrm{mcg} / \mathrm{kg} / \mathrm{min}$ for 3 minutes)

\begin{tabular}{|c|c|c|c|c|c|}
\hline $\begin{array}{l}\text { Healthy volunteers with low pretest } \\
\text { likelihood of coronary disease who } \\
\text { were undergoing CAG. }\end{array}$ & 14 & $62.6 \pm 9.1$ & $8 / 6$ & $\begin{array}{l}\text { TTE (CFR), distal } \\
\text { LAD with } \\
\text { pulse-wave } \\
\text { Doppler }\end{array}$ & $\begin{array}{l}\text { adenosine } \\
\text { ( } 140 \mathrm{mcg} / \mathrm{kg} / \mathrm{min} \\
\text { for } 5 \text { minutes) }\end{array}$ \\
\hline $\begin{array}{l}\text { Healthy volunteers, enrolled from } \\
\text { the non-medical hospital staff, } \\
\text { comparable in age and sex. }\end{array}$ & 10 & $54 \pm 8$ & $6 / 4$ & $\begin{array}{l}\text { TTE (CFR), } \\
\text { mid-distal LAD } \\
\text { with Doppler } \\
\text { spectral tracing }\end{array}$ & $\begin{array}{l}\text { adenosine } \\
\text { ( } 140 \mathrm{mcg} / \mathrm{kg} / \mathrm{min} \\
\text { for } 90 \text { seconds) }\end{array}$ \\
\hline $\begin{array}{l}\text { Healthy subjects, age and sex } \\
\text { matched. }\end{array}$ & 20 & $56 \pm 6$ & $12 / 8$ & $\begin{array}{l}\text { TTE (CFR), } \\
\text { mid-distal LAD } \\
\text { with Doppler } \\
\text { spectral tracing }\end{array}$ & $\begin{array}{l}\text { adenosine } \\
\text { (140 } \mathrm{mcg} / \mathrm{kg} / \mathrm{min} \\
\text { for } 90 \text { seconds) }\end{array}$ \\
\hline N/A & N/A & $\mathrm{N} / \mathrm{A}$ & N/A & CMR (MPRI), 1.5T & $\begin{array}{l}\text { adenosine } \\
(140 \mathrm{mcg} / \mathrm{kg} / \mathrm{min} \\
\text { for } 5 \mathrm{~min})\end{array}$ \\
\hline
\end{tabular}

\begin{tabular}{|c|c|c|c|c|c|}
\hline Healthy subjects & 13 & $\begin{array}{l}58(48-73) \\
\text { Median } \\
\text { (range) }\end{array}$ & $11 / 2$ & $\begin{array}{l}\text { PET (MPR), N-13 } \\
\text { ammonia }\end{array}$ & $\begin{array}{l}\text { adenosine } \\
(140 \mathrm{mcg} / \mathrm{kg} / \mathrm{min} \\
\text { for } 6 \text { minutes) }\end{array}$ \\
\hline $\begin{array}{l}\text { Healthy volunteers enrolled from } \\
\text { the nonmedical hospital staff, age } \\
\text { and sex matched. }\end{array}$ & 20 & $52 \pm 7$ & $11 / 9$ & $\begin{array}{l}\text { TTE (CBF), } \\
\text { mid-distal LAD } \\
\text { with Doppler } \\
\text { spectral tracing }\end{array}$ & $\begin{array}{l}\text { adenosine } \\
\text { (140 } \mathrm{mcg} / \mathrm{kg} / \mathrm{min} \\
\text { for } 90 \text { seconds) }\end{array}$ \\
\hline $\begin{array}{l}\text { Middle aged male-male twin pairs } \\
\text { from the Vietnam Era Twin Registry } \\
\text { without previous history of CAD. }\end{array}$ & 268 & $\begin{array}{l}54.0 \\
(53.5-54.6) \\
\text { Median } \\
\text { (range) }\end{array}$ & $0 / 268$ & $\begin{array}{l}\text { PET (CFR), N-13 } \\
\text { ammonia }\end{array}$ & $\begin{array}{l}\text { adenosine } \\
\text { (140 } \mathrm{mcg} / \mathrm{kg} / \mathrm{min} \\
\text { for } 4 \text { minutes) }\end{array}$ \\
\hline $\begin{array}{l}\text { Healthy subjects without } \\
\text { cardiovascular risk factors. }\end{array}$ & 27 & $41 \pm 13$ & $16 / 11$ & $\begin{array}{l}\text { PET (CFR), 150- } \\
\text { labeled water }\end{array}$ & $\begin{array}{l}\text { adenosine } \\
\text { (140 } \mathrm{mcg} / \mathrm{kg} / \mathrm{min} \\
\text { for } 3 \text { minutes) }\end{array}$ \\
\hline $\begin{array}{l}\text { Healthy subjects enrolled from } \\
\text { patients referred to outpatient } \\
\text { cardiology clinic for palpitations or } \\
\text { evaluation of cardiovascular risk, } \\
\text { age and sex matched. }\end{array}$ & 14 & $61 \pm 3$ & $7 / 7$ & $\begin{array}{l}\text { TTE (CBF), } \\
\text { mid-distal LAD } \\
\text { with Doppler } \\
\text { spectral tracing }\end{array}$ & $\begin{array}{l}\text { adenosine } \\
\text { (140 } \mathrm{mcg} / \mathrm{kg} / \mathrm{min} \\
\text { for } 90 \text { seconds) }\end{array}$ \\
\hline $\begin{array}{l}\text { Healthy individuals without } \\
\text { cardiovascular risk factors. }\end{array}$ & 14 & $58 \pm 6$ & $11 / 3$ & CMR (CFR), 3T & $\begin{array}{l}\text { adenosine ( } 140 \\
\mathrm{mcg} / \mathrm{kg} / \mathrm{min} \text { for } \\
4-5 \text { minutes) }\end{array}$ \\
\hline
\end{tabular}


Table 1. Continued

\begin{tabular}{|c|c|c|c|c|c|}
\hline $\begin{array}{l}\text { Author } \\
\text { (year) }\end{array}$ & Study design & Patient group & $\mathbf{N}=$ & $\begin{array}{l}\text { Mean age } \\
( \pm S D)\end{array}$ & $\begin{array}{l}\text { Sex } \\
\text { (F/M) }\end{array}$ \\
\hline $\begin{array}{l}\text { Uusitalo } \\
\text { (2013) }\end{array}$ & Cohort & $\mathrm{N} / \mathrm{A}$ & $\mathrm{N} / \mathrm{A}$ & $\mathrm{N} / \mathrm{A}$ & $\mathrm{N} / \mathrm{A}$ \\
\hline
\end{tabular}

\begin{tabular}{|c|c|c|c|c|c|}
\hline $\begin{array}{l}\text { Nelson } \\
(2014)\end{array}$ & Case-control & N/A & $\mathrm{N} / \mathrm{A}$ & N/A & $\mathrm{N} / \mathrm{A}$ \\
\hline $\begin{array}{l}\text { Thomson } \\
\text { (2015) }\end{array}$ & Case-control & $\begin{array}{l}\text { Women with signs and symptoms of ischemia } \\
\text { with clinically indicated CRT; part of } \\
\text { NHLBI-sponsored WISE-Coronary Vascular } \\
\text { Dysfunction study performed at Cedars-Sinai } \\
\text { Medical Center or the University of Florida. } \\
\text { Exclusion: history of obstructive CAD ( }>50 \% \\
\text { stenosis) or other cardiac disease, } \\
\text { contraindications to CMR. }\end{array}$ & 118 & $53.9 \pm 11.4$ & $118 / 0$ \\
\hline \multirow[t]{2}{*}{$\begin{array}{l}\text { Tagliamonte } \\
\text { (2015) }\end{array}$} & RCT & $\begin{array}{l}\text { Signs and symptoms of myocardial ischemia, } \\
\text { no CAD (<70\% stenosis on CAG). Myocardial } \\
\text { ischemia confirmed by SPECT, assigned to } \\
\text { placebo. }\end{array}$ & 29 & $65 \pm 11$ & $9 / 20$ \\
\hline & & $\begin{array}{l}\text { Exclusion: renal failure or hepatic } \\
\text { insufficiency, LBBB on ECG, use of drugs } \\
\text { inhibiting CYP3A, other cardiac disease. } \\
\text { As above, assigned to ranolazine. }\end{array}$ & 29 & $66 \pm 10$ & $10 / 19$ \\
\hline $\begin{array}{l}\text { Wu } \\
(2015)\end{array}$ & $\mathrm{RCT}$ & $\begin{array}{l}\text { Diagnosis of CMD based on the presence of } \\
\text { typical effort angina, exercise-induced ST } \\
\text { segment depression }(>1 \mathrm{~mm}) \text {, normal CAG, } \\
\text { absence of any specific cardiac disease } \\
\text { including vasospastic angina and reduced CFR } \\
(<2.0) \text { measured by TTE with adenosine. }\end{array}$ & 20 & $60 \pm 8$ & $17 / 3$ \\
\hline $\begin{array}{l}\text { Bairey Merz } \\
(2016)\end{array}$ & $\mathrm{RCT}$ & $\begin{array}{l}\text { Symptoms due to ischemia objectified by } \\
\text { stress testing, no obstructive CAD ( }<50 \% \\
\text { stenosis on CAG) with abnormal CRT (CFR } \\
<2.5 \text { ) or CMR (MPRI }<2.0 \text { ). Exclusion: other } \\
\text { cardiac disease or life expectancy }<4 \text { years, } \\
\text { contraindication for CMR or use of CYP3A4 } \\
\text { inhibitors. }\end{array}$ & 128 & $55.2 \pm 9.8$ & $123 / 5$ \\
\hline $\begin{array}{l}\text { Bakir } \\
(2016)\end{array}$ & Case series & $\mathrm{N} / \mathrm{A}$ & $\mathrm{N} / \mathrm{A}$ & $\mathrm{N} / \mathrm{A}$ & $\mathrm{N} / \mathrm{A}$ \\
\hline
\end{tabular}


$\mathbf{N}=$ Mean age
$( \pm$ SD)

$35.3 \pm 3.9$

Healthy men $\leq 45$ years from healthy 77 control groups of two earlies reported studies. Exclusion: hypertension, smoking, diabetes mellitus, obesity or history of atherosclerotic disease.

Healthy age matched women with
no cardiac risk factors.

Healthy age matched women with

Healthy age matched women with no cardiac risk factors.

56 (SD not
available)

\section{Sex Imaging modality Vasoactive agent \\ (F/M) (outcome \\ parameter)}

0/77 PET (CFR),

150-labeled water

adenosine (dose

not reported)

or dipyridamole

( $0.56 \mathrm{mg} / \mathrm{kg}$ per 4 minutes)

\section{$15 / 0$

$15 / 0$

CMR (MPRI), 1.5T

$53.6 \pm 9.1$

$21 / 0$

CMR (MPRI), $1.5 T$

adenosine

(140 mcg/kg 3-4 minutes)

adenosine

$(140 \mathrm{mcg} / \mathrm{kg}$ from 2 min prior until completion of first pass perfusion imaging)

\title{
N/A
}

N/A N/A

$\begin{array}{ccll}\text { N/A N/A N/A } & \text { N/A } & \text { TTE (CBFVR), } & \text { nitroglycerin } \\ & \text { mid-distal LAD } & \text { (25 mcg) } \\ & & \text { with Doppler } \\ & \text { spectral tracing }\end{array}$

$\begin{array}{ccll}\text { N/A N/A N/A } & \text { N/A } & \text { TTE (CBFVR), } & \text { nitroglycerin } \\ & \text { mid-distal LAD } & \text { (25 mcg) } \\ & & \text { with Doppler } \\ & \text { spectral tracing }\end{array}$

TTE (CBFVR), mid-distal LAD

with Doppler nitroglycerin spectral tracing

\section{N/A}

N/A N/A

N/A

CMR (MPRI), 1.5T

adenosine

(not reported)

\begin{abstract}
Women without signs and and absence of cardiovascular risk

factors recruited at Cedars-Sinai

Medical Center based on their age and hormone-use status to match CMD subjects in the WISE trial.

Exclusion: contraindication to $\mathrm{CMR}$ or adenosine, renal disease.
\end{abstract}

$20 \quad 54 \pm 9$

$20 / 0$

CMR (MPRI), 1.5T

adenosine (140 mcg/kg/min for 3-4 minutes) 
Table 1. Continued

\begin{tabular}{|c|c|c|c|c|c|}
\hline $\begin{array}{l}\text { Author } \\
\text { (year) }\end{array}$ & Study design & Patient group & $N=$ & $\begin{array}{l}\text { Mean age } \\
( \pm S D)\end{array}$ & $\begin{array}{l}\text { Sex } \\
\text { (F/M) }\end{array}$ \\
\hline $\begin{array}{l}\text { Mygind } \\
(2016)\end{array}$ & Case series & $\begin{array}{l}\text { Women referred for clinically indicated CAG } \\
\text { due to angina-like chest pain form the Patient } \\
\text { Analysis \& Tracking System in eastern } \\
\text { Denmark. Inclusion: CAD }<50 \% \text { stenosis. } \\
\text { Exclusion: other cause of chest pain more } \\
\text { likely, no cardiac disease, life-expectancy }<1 \\
\text { year. }\end{array}$ & 963 & $62.1 \pm 9.7$ & $963 / 0$ \\
\hline $\begin{array}{l}\text { Anchisi } \\
\text { (2017) }\end{array}$ & Case series & $\begin{array}{l}\text { Recurrent chest pain, ECG alterations at } \\
\text { ergometry and normal CAG. Exclusion: other } \\
\text { cardiac disease and previous } \\
\text { revascularization. Setting: Cardiology Unit of } \\
\text { Azienda Ospedaliera-Universitaria 'Maggiore } \\
\text { della Carità' in Novara. }\end{array}$ & 16 & $64 \pm 11$ & $10 / 6$ \\
\hline $\begin{array}{l}\text { Jaarsma } \\
\text { (2017) }\end{array}$ & Case-control & $\begin{array}{l}\text { Typical effort angina, positive stress ECG and } \\
\text { normal CAG (stenosis }<25 \%) \text {, consecutively } \\
\text { enrolled at Maastricht University Medical } \\
\text { Center. Exclusion: contraindications for CMR } \\
\text { or adenosine. One patient excluded due to } \\
\text { poor image quality. }\end{array}$ & 13 & $65 \pm 9$ & $7 / 6$ \\
\hline $\begin{array}{l}\text { Michelsen } \\
\text { (2017) }\end{array}$ & Case-control & $\begin{array}{l}\text { Women with angina-like chest pain and no } \\
\text { significant obstructive CAD ( }<50 \% \text { stenosis) } \\
\text { and with successful TTE examination, } \\
\text { randomly selected from the iPOWER study } \\
\text { cohort. }\end{array}$ & $\begin{array}{l}95 \\
102\end{array}$ & $\begin{array}{l}61.8 \pm 8.8 \\
\text { (in all } 107 \\
\text { particpants) }\end{array}$ & $102 / 0$ \\
\hline
\end{tabular}

\begin{tabular}{|c|c|c|c|c|c|}
\hline $\begin{array}{l}\text { Liu } \\
(2018)\end{array}$ & Case-control & $\begin{array}{l}\text { Patients with angina and suspected or known } \\
\text { CAD referred for outpatient diagnostic CAG } \\
\text { without obstructive CAD on CAG. }\end{array}$ & 22 & $65 \pm 8$ & $8 / 14$ \\
\hline $\begin{array}{l}\text { Liu } \\
(2018)\end{array}$ & Case-control & $\begin{array}{l}\text { Patients with stable angina and suspected } \\
C A D \text { referred for outpatient diagnostic CAG in } \\
\text { a tertiary referral hospital with FFR } \geq 0.8 \text { and } \\
\text { IMR } \geq 25 \mathrm{U} \text {. }\end{array}$ & $\begin{array}{l}13 \\
11\end{array}$ & N/A & N/A \\
\hline $\begin{array}{l}\text { Zorach } \\
(2018)\end{array}$ & Case-control & $\begin{array}{l}\text { Patients with typical effort angina and no CAD } \\
\text { ( }<50 \% \text { stenosis) on CAG and with risk factors } \\
\text { for CMD (diabetes mellitus or metabolic } \\
\text { syndrome) recruited from the University of } \\
\text { Virginia Health System. }\end{array}$ & 46 & $57.5 \pm 11.2$ & $34 / 12$ \\
\hline $\begin{array}{l}\text { Rahman } \\
(2019)\end{array}$ & Case-control & $\begin{array}{l}\text { Patients undergoing elective diagnostic } \\
\text { angiography for investigation of exertional } \\
\text { chest pain and nonobstructive coronary } \\
\text { artery disease ( }<30 \% \text { diameter stenosis and/ } \\
\text { or fractional flow reserve }>0.80 \text { ) with CFR }< \\
2.5 \text {. }\end{array}$ & 38 & $2.01 \pm 0.41$ & $\mathrm{~N} / \mathrm{A}$ \\
\hline
\end{tabular}

CABG, coronary artery bypass grafting; $C A D$, coronary artery disease; $C A G$, coronary angiography; $C R T$, coronary reactivity testing; ECG, electrocardiogram; F, female; FFR, fractional flow reserve; IMR, index of microcirculatory resistance; ISMN, isosorbide-5mononitrate; LAD, left anterior descending coronary artery; LBBB, left bundle branch block; M, male; NHLBI-sponsored WISE. National Heart, Lung, and Blood Institute sponsored women's ischemia syndrome evaluation; RCT, randomized controlled trial 


\begin{tabular}{|c|c|c|c|c|c|}
\hline Control group & $\mathbf{N}=$ & $\begin{array}{l}\text { Mean age } \\
( \pm S D)\end{array}$ & $\begin{array}{l}\text { Sex } \\
\text { (F/M) }\end{array}$ & $\begin{array}{l}\text { Imaging modality } \\
\text { (outcome } \\
\text { parameter) }\end{array}$ & $\begin{array}{l}\text { Vasoactive agent } \\
\text { used (dose) }\end{array}$ \\
\hline N/A & N/A & N/A & $\mathrm{N} / \mathrm{A}$ & $\begin{array}{l}\text { TTE (CFVR), LAD } \\
\text { with pulsed-wave } \\
\text { Doppler } \\
\text { Contrast } \\
\text { (SonoVue) used in } \\
\text { case of difficulty } \\
\text { visualizing LAD. }\end{array}$ & $\begin{array}{l}\text { dipyridamole } \\
(0.84 \mathrm{mg} / \mathrm{kg} \text { in } 6 \\
\mathrm{min})\end{array}$ \\
\hline $\mathrm{N} / \mathrm{A}$ & $\mathrm{N} / \mathrm{A}$ & $\mathrm{N} / \mathrm{A}$ & $\mathrm{N} / \mathrm{A}$ & $\begin{array}{l}\text { TTE (CFR), color } \\
\text { Doppler flow } \\
\text { mapping, } \\
\text { mid-distal LAD }\end{array}$ & $\begin{array}{l}\text { dipyridamole } \\
(0.84 \mathrm{mg} / \mathrm{kg} \text { per } 6 \\
\mathrm{min})\end{array}$ \\
\hline $\mathrm{N} / \mathrm{A}$ & $\mathrm{N} / \mathrm{A}$ & $\mathrm{N} / \mathrm{A}$ & $\mathrm{N} / \mathrm{A}$ & CMR (MPR), 3T & $\begin{array}{l}\text { adenosine } \\
(140 \mathrm{mcg} / \mathrm{kg} / \mathrm{min} \\
\text { for } 4 \mathrm{~min})\end{array}$ \\
\hline $\mathrm{N} / \mathrm{A}$ & $\mathrm{N} / \mathrm{A}$ & $\mathrm{N} / \mathrm{A}$ & $\mathrm{N} / \mathrm{A}$ & $\begin{array}{l}\text { PET (MBFR), } \\
\text { Rubidium-82; } \\
\text { TTE (CFVR), LAD } \\
\text { with pulse-waved } \\
\text { Doppler; Contrast } \\
\text { (SonoVue) used in } \\
\text { case of difficulty } \\
\text { visualizing LAD. }\end{array}$ & $\begin{array}{l}\text { adenosine } \\
(0.84 \mathrm{mg} / \mathrm{kg} \text { per } 6 \\
\mathrm{min}) \\
\text { dipyridamole } \\
(0.84 \mathrm{mg} / \mathrm{kg} \text { per } 6 \\
\mathrm{min})\end{array}$ \\
\hline Healthy age-matched subjects. & 20 & $61 \pm 7$ & $7 / 13$ & $\begin{array}{l}\text { CMR (MPRI), 1.5T } \\
\text { or } 3 T\end{array}$ & $\begin{array}{l}\text { adenosine } \\
(140 \mathrm{mcg} / \mathrm{kg} / \mathrm{min} \\
\text { for } \geq 3 \text { to } 6 \mathrm{~min})\end{array}$ \\
\hline Healthy volunteers & 30 & $51 \pm 15$ & $9 / 21$ & CMR, 1.5 or $3 T$ & $\begin{array}{l}\text { adenosine } \\
(140 \mathrm{mcg} / \mathrm{kg} / \mathrm{min} \text {, } \\
\text { for } \geq 3 \text { to } 6 \mathrm{~min})\end{array}$ \\
\hline $\begin{array}{l}\text { Healthy controls without risk factors } \\
\text { for CMD. }\end{array}$ & 20 & $53.4 \pm 11.9$ & $12 / 8$ & CMR (MPR), 1.5T & regadenoson \\
\hline $\begin{array}{l}\text { Patients undergoing elective } \\
\text { diagnostic angiography for } \\
\text { investigation of exertional chest } \\
\text { pain and nonobstructive coronary } \\
\text { artery disease ( }<30 \% \text { diameter } \\
\text { stenosis and/or fractional flow } \\
\text { reserve }>0.80 \text { ) with CFR }>2.5 \text {. }\end{array}$ & 27 & $2.68 \pm 0.49$ & $\mathrm{~N} / \mathrm{A}$ & $\mathrm{CMR}$ (MPR), 3T & $\begin{array}{l}\text { adenosine } \\
(140 \mathrm{mcg} / \mathrm{kg} / \mathrm{min} \\
\text { for } 3 \mathrm{~min})\end{array}$ \\
\hline
\end{tabular}




\section{SUPPLEMENTARY MATERIAL}

\section{Additional file 1: Search Strategy}

\section{PubMed search strategy}

(((nonocclusive OR "non occlusive" OR nonobstructive OR "non obstructive" OR "microvascular dysfunction" OR "small vessel disease" OR "cardiac syndrome X" OR "microvascular angina" OR CMD OR MVD)) AND (coronary OR heart OR myocardial OR cardiac)) AND (imaging OR MRI OR CT OR SPECT OR PET OR TTE OR echo)

\section{Embase search strategy}

(nonocclusive $O R$ 'non occlusive' $O R$ nonobstructive $O R$ 'non obstructive' $O R$ 'microvascular dysfunction' $O R$ 'small vessel disease' $O R$ 'cardiac syndrome $x$ ' $O R$ 'microvascular angina' OR cmd OR mvd) AND (coronary OR heart OR myocardial OR cardiac) AND (imaging OR mri OR ct OR spect OR pet OR tte OR echo) 


\section{Additional file 2}

Table S1. Risk of bias assessment according to QUADAS-2

\begin{tabular}{|c|c|c|c|c|c|c|c|c|c|}
\hline \multirow[t]{2}{*}{ Author (year) } & \multicolumn{5}{|c|}{ Risk of bias } & \multicolumn{4}{|c|}{ Applicability concerns } \\
\hline & 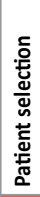 & 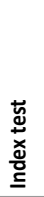 & 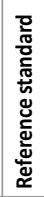 & 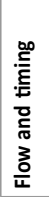 & 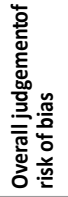 & 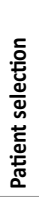 & 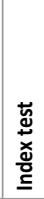 & 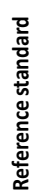 & 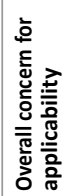 \\
\hline \multicolumn{10}{|l|}{ Meeder (1997) } \\
\hline \multicolumn{10}{|l|}{ Bottcher (1999) } \\
\hline \multicolumn{10}{|l|}{ Buus (1999) } \\
\hline \multicolumn{10}{|l|}{ Panting (2002) } \\
\hline \multicolumn{10}{|l|}{ Marroquin (2003) } \\
\hline \multicolumn{10}{|l|}{ De Vries (2006) } \\
\hline \multicolumn{10}{|l|}{ Graf (2006) } \\
\hline \multicolumn{10}{|l|}{ Pärkkä (2006) } \\
\hline \multicolumn{10}{|l|}{ Wöhrle (2006) } \\
\hline \multicolumn{10}{|l|}{ Graf (2007) } \\
\hline \multicolumn{10}{|l|}{ Vermeltfoort (2007) } \\
\hline \multicolumn{10}{|l|}{ Galiuto (2007) } \\
\hline \multicolumn{10}{|l|}{ Cemin (2008) } \\
\hline \multicolumn{10}{|l|}{ Lanza (2008) } \\
\hline \multicolumn{10}{|l|}{ Di Monaco (2009) } \\
\hline \multicolumn{10}{|l|}{ Mehta (2011) } \\
\hline \multicolumn{10}{|l|}{ Scholtens (2011) } \\
\hline \multicolumn{10}{|l|}{ Sestito (2011) } \\
\hline \multicolumn{10}{|l|}{ Vaccarino (2011) } \\
\hline \multicolumn{10}{|l|}{ Vermeltfoort (2011) } \\
\hline \multicolumn{10}{|l|}{ Di Franco (2012) } \\
\hline \multicolumn{10}{|l|}{ Karamitsos (2012) } \\
\hline Uusitalo (2013) & & & & & & & & & \\
\hline Nelson (2014) & & & & & & & & & \\
\hline Thomson (2015) & & & & & & & & & \\
\hline Tagliamonte (2015) & & & & & & & & & \\
\hline Wu (2015) & & & & & & & & & \\
\hline Bairey Merz (2016) & & & & & & & & & \\
\hline Bakir (2016) & & & & & & & & & \\
\hline Mygind (2016) & & & & & & & & & \\
\hline Anchisi (2017) & & & & & & & & & \\
\hline Jaarsma (2017) & & & & & & & & & \\
\hline Michelsen (2017) & & & & & & & & & \\
\hline Liu (2018) (19) & & & & & & & & & \\
\hline Liu (2018) (27) & & & & & & & & & \\
\hline Zorach (2018) & & & & & & & & & \\
\hline Rahman (2019) & & & & & & & & & \\
\hline
\end{tabular}

Legenda risk of bias assessment

Low risk of bias or Low concern of applicability High risk of bias or High concern of applicability Unclear risk of bias or Unclear concern of applicability 


\section{Additional file 3}

Table S2. Quantification of coronary microvascular dysfunction in CMR studies

\begin{tabular}{|c|c|c|c|c|c|c|}
\hline Study & Outcome & Protocol & $\begin{array}{l}\text { Patients } \\
\text { (n) }\end{array}$ & Mean ( $\pm S D$ ) & $\begin{array}{l}\text { Controls } \\
\text { (n) }\end{array}$ & Mean ( $\pm S D$ ) \\
\hline Panting (2002) & MPRI & 1.5T, adenosine & 20 & $1.47 \pm 0.36$ & 10 & $1.50 \pm 0.47$ \\
\hline Wöhrle (2006) & MPRI & $1.5 \mathrm{~T}$, adenosine & 12 & $1.48 \pm 0.71$ & N/A & N/A \\
\hline Pärkkä (2006) & MPR & $1.5 \mathrm{~T}$, dipyridamole & $N / A$ & N/A & 18 & $2.51 \pm 0.95$ \\
\hline Vermeltfoort (2007) & MPRI & $1.5 \mathrm{~T}$, adenosine & 20 & $1.83 \pm 0.50$ & N/A & $\mathrm{N} / \mathrm{A}$ \\
\hline Karamitsos (2012) & $\begin{array}{l}\text { CFR (correct } \\
\text { ed for RPP) }\end{array}$ & 3.0T, adenosine & 18 & $2.63 \pm 0.12$ & 14 & $2.53 \pm 0.13$ \\
\hline Nelson (2014) & MPRI & $1.5 \mathrm{~T}$, adenosine & N/A & N/A & 15 & $2.20 \pm 0.53$ \\
\hline Thomson (2015) & MPRI & $1.5 \mathrm{~T}$, adenosine & 118 & $1.71 \pm 0.43$ & 21 & $2.23 \pm 0.37$ \\
\hline Bairey Merz (2016) & MPRI & $1.5 \mathrm{~T}$, adenosine & 128 & $1.60 \pm 0.30$ & N/A & $\mathrm{N} / \mathrm{A}$ \\
\hline Bakir (2016) & MPRI & $1.5 \mathrm{~T}$, adenosine & N/A & N/A & 20 & $2.19 \pm 0.38$ \\
\hline Liu (2018) (15) & MPRI & $\begin{array}{l}1.5 \mathrm{~T} \text { or } 3.0 \mathrm{~T}, \\
\text { adenosine }\end{array}$ & 22 & $1.60 \pm 0.50$ & 20 & $2.00 \pm 0.30$ \\
\hline \multirow[t]{2}{*}{ Liu (2018) (29) } & $\begin{array}{l}\text { MPRI } \\
\text { (MPR) }\end{array}$ & $1.5 \mathrm{~T}$, adenosine & 13 & $\begin{array}{l}1.30 \pm 0.50 \\
(1.50 \pm 0.60)\end{array}$ & 20 & $\begin{array}{l}2.00 \pm 0.30 \\
(2.80 \pm 0.50)\end{array}$ \\
\hline & & 3.0T, adenosine & 11 & $\begin{array}{l}1.20 \pm 0.20 \\
(1.80 \pm 0.70)\end{array}$ & 10 & $\begin{array}{l}2.00 \pm 0.30 \\
(2.80 \pm 0.40)\end{array}$ \\
\hline Rahman (2019) & MPR & 3.0T, adenosine & 38 & $2.01 \pm 0.41$ & 27 & $2.68 \pm 0.49$ \\
\hline \multicolumn{7}{|l|}{ Median (IQR) } \\
\hline Jaarsma (2017) & MPR & 3.0T, adenosine & 12 & 1.35 (1.09-1.78) & $\mathrm{N} / \mathrm{A}$ & $\mathrm{N} / \mathrm{A}$ \\
\hline \multicolumn{7}{|l|}{ Median (range) } \\
\hline Mehta (2011) & MPRI & $1.5 \mathrm{~T}$, adenosine & 20 & $1.40(1.20-1.90)$ & N/A & $\mathrm{N} / \mathrm{A}$ \\
\hline Zorach (2018) & MPR & $1.5 \mathrm{~T}$, regadenoson & 46 & $2.21(1.95-2.69)$ & 20 & $2.93(2.76-3.19)$ \\
\hline
\end{tabular}




\section{Additional file 4}

Table S3. Quantification of coronary microvascular dysfunction in PET studies. Correction for RPP is indicated by + or -

\begin{tabular}{|c|c|c|c|c|c|c|}
\hline Study & Outcome & Protocol & $\begin{array}{l}\text { Patients } \\
\text { (n) }\end{array}$ & Mean ( $\pm S D)$ & $\begin{array}{l}\text { Controls } \\
\text { (n) }\end{array}$ & Mean ( $\pm S D)$ \\
\hline Meeder (1997) & MPR (-) & $\mathrm{N}-13$, dipyridamole & 25 & $2.71 \pm 0.67$ & 21 & $2.91 \pm 1.04$ \\
\hline Bottcher (1999) & CFR $(+)$ & $\mathrm{N}-13$, dipyridamole & 25 & $2.03 \pm 0.53$ & 15 & $2.96 \pm 0.63$ \\
\hline Marroquin (2003) & CFR (-) & $\mathrm{N}-13$, adenosine & 34 & $2.85 \pm 1.35$ & $\mathrm{~N} / \mathrm{A}$ & N/A \\
\hline Graf (2006) & CFR $(+)$ & $\mathrm{N}-13$, dipyridamole & 58 & $2.14 \pm 0.88$ & $\mathrm{~N} / \mathrm{A}$ & N/A \\
\hline De Vries (2006) & CFR (+) & $\mathrm{N}-13$, dipyridamole & 24 & $1.99 \pm 0.70$ & 21 & $2.91 \pm 1.04$ \\
\hline Pärkkä (2006) & MPR (-) & $\begin{array}{l}\text { 15-O labeled water, } \\
\text { dipyridamole }\end{array}$ & N/A & N/A & 18 & $4.32 \pm 1.78$ \\
\hline Graf (2007) & CFR (-) & $\mathrm{N}-13$, dipyridamole & 79 & $2.37 \pm 1.00$ & $\mathrm{~N} / \mathrm{A}$ & N/A \\
\hline Scholtens (2011) & MPR (+) & $\mathrm{N}-13$, adenosine & 14 & $1.39 \pm 0.31$ & 13 & $2.91 \pm 0.78$ \\
\hline Vaccarino (2011) & CFR (-) & $\mathrm{N}-13$, adenosine & $\mathrm{N} / \mathrm{A}$ & N/A & 268 & $2.68 \pm 0.83$ \\
\hline Vermeltfoort (2011) & CFR $(+)$ & $\begin{array}{l}\text { 15-O labeled water, } \\
\text { adenosine }\end{array}$ & N/A & N/A & 27 & $3.28 \pm 0.83$ \\
\hline Uusitalo (2013) & CFR $(+)$ & $\begin{array}{l}\text { 15-O labeled water, } \\
\text { adenosine or dipyridamole }\end{array}$ & N/A & $\mathrm{N} / \mathrm{A}$ & 77 & $4.10 \pm 1.30$ \\
\hline \multicolumn{7}{|l|}{ Mean \pm SEM } \\
\hline Buus (1999) & CFR (-) & $\mathrm{N}-13$, dipyridamole & 16 & $2.06 \pm 0.14$ & 15 & $2.93 \pm 0.17$ \\
\hline \multicolumn{7}{|l|}{ Median (IQR) } \\
\hline Michelsen (2017) & $\operatorname{MFR}(+)$ & $\mathrm{Rb}-82$, adenosine & 95 & $2.13(1.80-2.40)$ & $\mathrm{N} / \mathrm{A}$ & $\mathrm{N} / \mathrm{A}$ \\
\hline
\end{tabular}




\section{Additional file 5}

Table S4. Quantification of coronary microvascular dysfunction in TTE studies

\begin{tabular}{|c|c|c|c|c|c|c|}
\hline Study & Outcome & Protocol & Patients (n) & Mean ( \pm SD) & $\begin{array}{l}\text { Controls } \\
\text { (n) }\end{array}$ & Mean ( $\pm S D$ ) \\
\hline Galiuto (2007) & CFR & $\begin{array}{l}\text { PWDoppler, } \\
\text { distal LAD, } \\
\text { adenosine }\end{array}$ & 17 & $1.98 \pm 0.60$ & 17 & $2.88 \pm 1.50$ \\
\hline Cemin (2008) & CFR & $\begin{array}{l}\text { PWDoppler, } \\
\text { distal LAD, } \\
\text { adenosine }\end{array}$ & $\mathrm{N} / \mathrm{A}$ & $N / A$ & 14 & $2.65 \pm 0.65$ \\
\hline Lanza (2008) & CFR & $\begin{array}{l}\text { Doppler ST, } \\
\text { mid-distal LAD, } \\
\text { adenosine }\end{array}$ & 18 & $2.03 \pm 0.60$ & 10 & $3.29 \pm 1.00$ \\
\hline Di Monaco (2009) & CFR & $\begin{array}{l}\text { Doppler ST, } \\
\text { mid-distal LAD, } \\
\text { adenosine }\end{array}$ & 29 & $1.94 \pm 0.63$ & 12 & $3.31 \pm 1.10$ \\
\hline Sestito (2011) & $\mathrm{CBF}$ & $\begin{array}{l}\text { Doppler ST, } \\
\text { mid-distal LAD, } \\
\text { adenosine }\end{array}$ & 71 & $2.05 \pm 0.60$ & 20 & $2.92 \pm 0.90$ \\
\hline Di Franco (2012) & $\mathrm{CBF}$ & $\begin{array}{l}\text { Doppler ST, } \\
\text { mid-distal LAD, } \\
\text { adenosine }\end{array}$ & 14 & $1.90 \pm 0.50$ & 14 & $3.10 \pm 0.90$ \\
\hline Tagliamonte (2015) & CFR & $\begin{array}{l}\text { Doppler ST, } \\
\text { mid-distal LAD, } \\
\text { dipyridamole }\end{array}$ & $\begin{array}{l}29 \text { (ranolazine) } \\
29 \text { (placebo) }\end{array}$ & $\begin{array}{l}1.91 \pm 0.31 \\
1.94 \pm 0.29\end{array}$ & $N / A$ & N/A \\
\hline Wu (2015) & CBFV & $\begin{array}{l}\text { PWDoppler, } \\
\text { mid-distal LAD, } \\
\text { nitroglycerin }\end{array}$ & $\begin{array}{l}13 \\
\text { (placebo) } 11 \\
\text { (ISMN) }\end{array}$ & $\begin{array}{l}1.69 \pm 0.40 \\
1.71 \pm 0.40\end{array}$ & N/A & N/A \\
\hline Anchisi (2017) & CFR & $\begin{array}{l}\text { Color Doppler, } \\
\text { mid-distal LAD, } \\
\text { dipyridamo le }\end{array}$ & 16 & $2.40 \pm 0.40$ & $N / A$ & N/A \\
\hline \multicolumn{7}{|l|}{ Median (IQR) } \\
\hline Mygind (2016) & CFVR & $\begin{array}{l}\text { PW Doppler, } \\
\text { LAD, dipyridamole } \\
\text { or MCE }\end{array}$ & 963 & $2.33(1.98-2.76)$ & $\mathrm{N} / \mathrm{A}$ & $\mathrm{N} / \mathrm{A}$ \\
\hline Michelsen (2017) & CFVR & $\begin{array}{l}\text { PW Doppler, } \\
\text { LAD, dipyridamole } \\
\text { or MCE }\end{array}$ & 102 & $1.99(1.64-2.43)$ & $N / A$ & $N / A$ \\
\hline
\end{tabular}




\section{Additional file 6}

Table S5. Definition of nonobstructive coronary artery disease in included studies

\begin{tabular}{|c|c|}
\hline Author (year) & Definition nonobstructive coronary artery disease (NOCAD) \\
\hline Meeder (1997) & Completely normal coronary arteries without any vessel irregularities on coronary angiography \\
\hline Bottcher (1999) & Completely normal coronary arteriography \\
\hline Buus (1999) & Normal coronary angiography with smooth coronary arteries \\
\hline Panting (2002) & $\begin{array}{l}\text { Completely normal results on coronary angiography, with no inducible spasm on ergonovine- } \\
\text { provocation testing }\end{array}$ \\
\hline Marroquin (2003) & Normal or minimal luminal irregularities on coronary angiography ( $<50 \%$ stenosis) \\
\hline De Vries (2006) & Normal coronary findings on angiography \\
\hline Graf (2006) & Normal angiogram \\
\hline Pärkkä (2006) & N/A \\
\hline Wöhrle (2006) & Smooth vessel contours without lumen irregularities on coronary angiography \\
\hline Galiuto (2007) & Angiographically normal coronary arteries \\
\hline Graf (2007) & Normal coronary angiogram \\
\hline Vermeltfoort (2007) & Completely normal results from coronary angiography \\
\hline Cemin (2008) & N/A \\
\hline Lanza (2008) & Angiographically normal epicardial coronary arteries \\
\hline Di Monaco (2009) & Totally normal coronary arteries at angiography \\
\hline Mehta (2011) & $<50 \%$ epicardial coronary stenosis in all epicardial coronary arteries on coronary angiography \\
\hline Scholtens (2011) & Normal coronary angiogram \\
\hline Sestito (2011) & $\begin{array}{l}\text { Smooth coronary arteries at angiography. Coronary artery spasm had been excluded according } \\
\text { to intracoronary or systemic ergonovine test in patients who reported angina episodes also at } \\
\text { rest }\end{array}$ \\
\hline Vaccarino (2011) & N/A \\
\hline Vermeltfoort (2011) & N/A \\
\hline Di Franco (2012) & Angiographically normal coronary arteries \\
\hline Karamitsos (2012) & Angiographically smooth normal epicardial coronary arteries on coronary angiography \\
\hline Uusitalo (2013) & N/A \\
\hline Nelson (2014) & $\mathrm{N} / \mathrm{A}$ \\
\hline Thomson (2015) & Exclusion: $>50 \%$ luminal diameter stenosis in 1 epicardial coronary artery \\
\hline Tagliamonte (2015) & $<70 \%$ coronary stenosis in all epicardial coronary arteries on coronary angiography \\
\hline Wu (2015) & Normal coronary angiography \\
\hline Bairey Merz (2016) & $<50 \%$ epicardial coronary stenosis in all epicardial coronary arteries \\
\hline Bakir (2016) & $\mathrm{N} / \mathrm{A}$ \\
\hline Mygind (2016) & $<50 \%$ stenosis of epicardial vessels assessed by diagnostic invasive coronary angiography \\
\hline Anchisi (2017) & Normal coronary angiography \\
\hline Jaarsma (2017) & $\begin{array}{l}\text { Normal coronary arteries or minimal vessel wall irregularities ( }<25 \% \text { stenosis) on coronary } \\
\text { angiography }\end{array}$ \\
\hline Michelsen (2017) & $<50 \%$ stenosis on coronary angiography \\
\hline Liu (2018) (19) & Angiographic NOCAD (<50\% visual stenosis) on coronary angiography \\
\hline Liu (2018) (27) & FFR $\geq 0.8$ and IMR $\geq 25 U$ measured with invasive coronary angiography \\
\hline Zorach (2018) & $\begin{array}{l}\text { Exclusion: }>50 \% \text { stenosis in at least one coronary artery or FFR }<0.8 \text { in at least one coronary } \\
\text { artery on coronary angiography }\end{array}$ \\
\hline
\end{tabular}



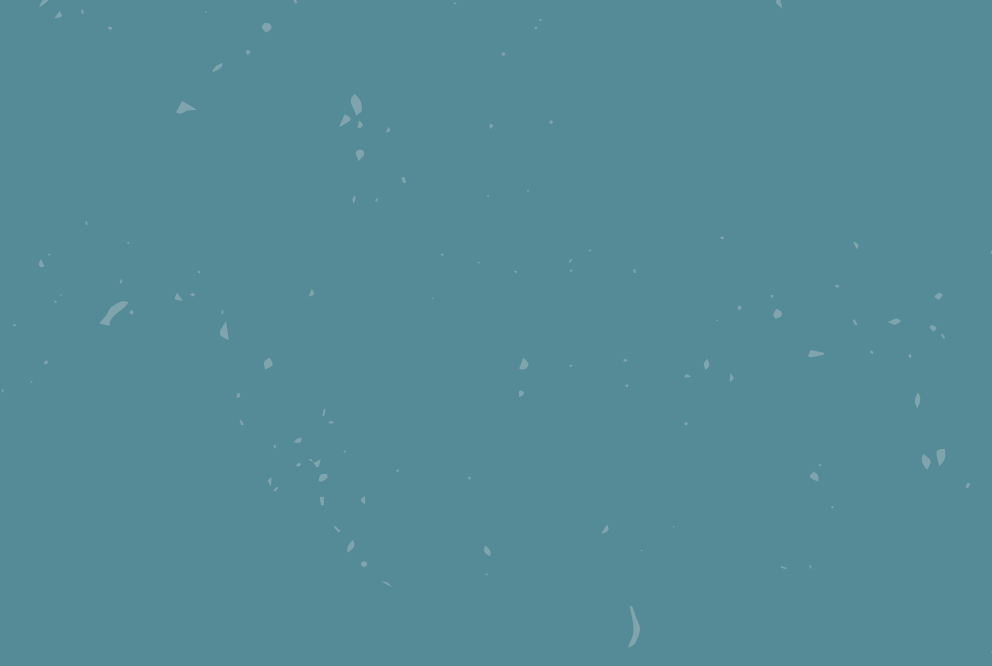

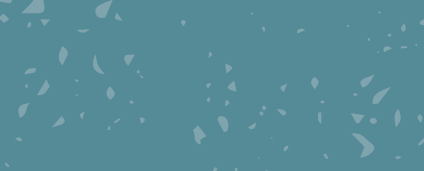




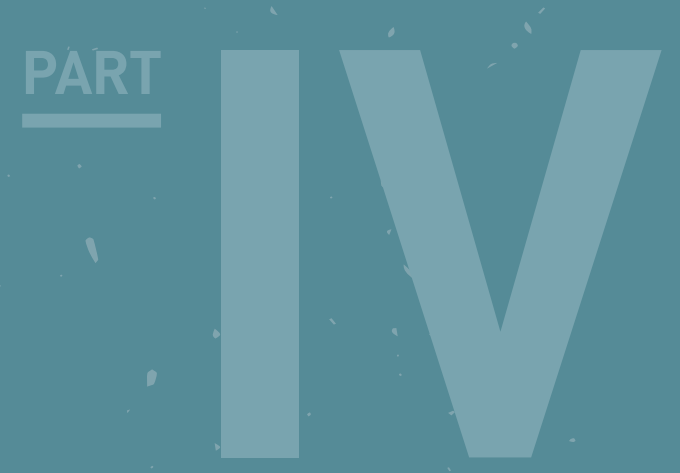

Summary and General Discussion:

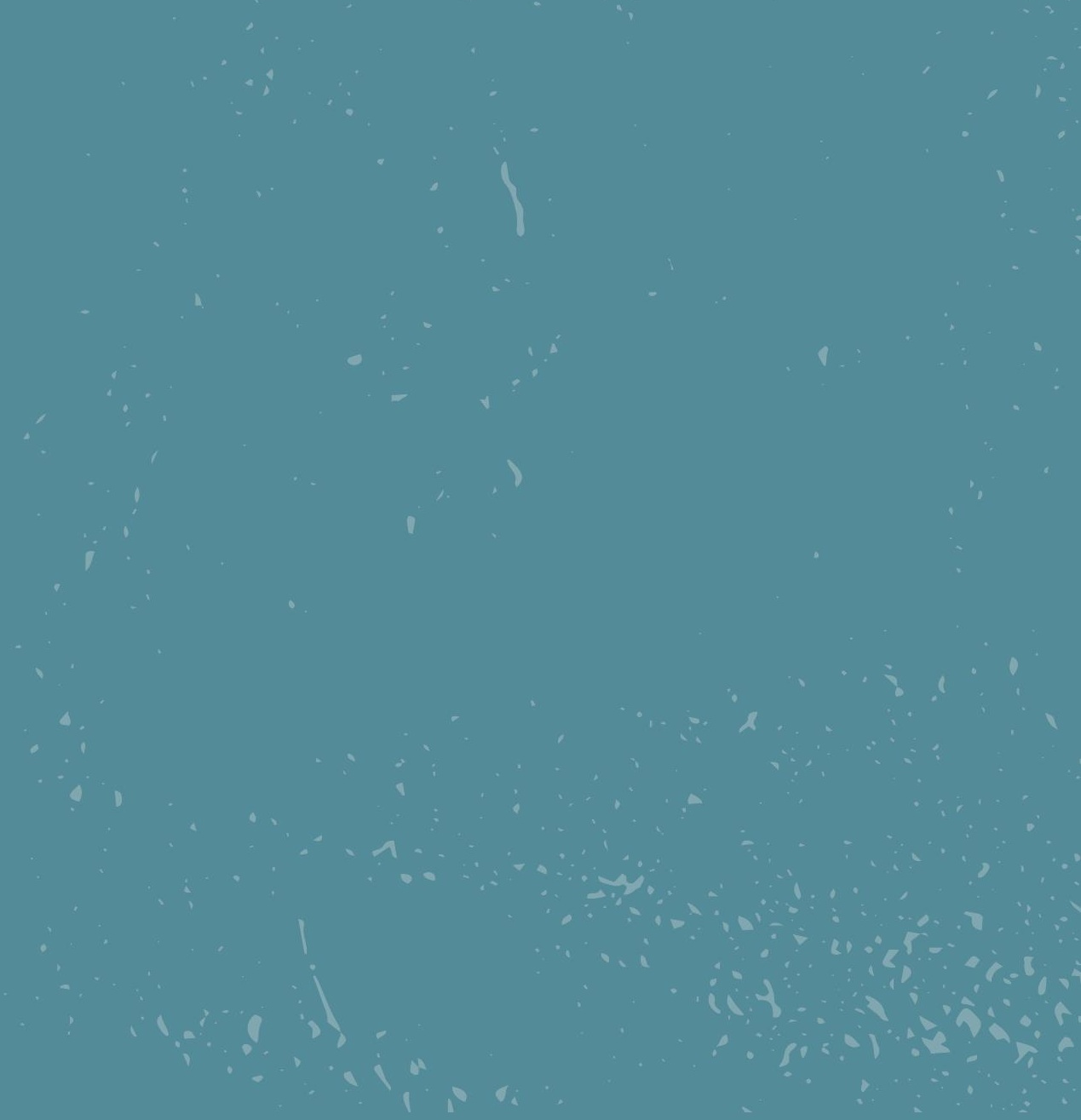


General Discussion 
Coronary vascular disease is still the number one cause of death in both women and men globally (1). However, the majority of coronary vascular disease research has been performed in men. Many findings from these studies have not yet been replicated in women, not even when the study in question had a balanced study population with equal numbers of women and men. As such, much of the contemporary evidence base for medicine still heavily relies on "male-biased" research. For chest pain patients this is apparent from the evaluation being primarily directed towards the in- or exclusion of the "typical male" form of coronary vascular disease; obstructive coronary artery disease (CAD). This approach seems outdated considering the current evolution of sex-specific research, revealing sex differences in risk factors, symptoms and development of coronary vascular disease, which has been emerging over the recent years. For example, we now know that, beyond obstructive $C A D$, there is also the risk of non-obstructive coronary vascular disease. This is functionally significant and thus relevant and seems to be more prevalent in women. Alas, this new evidence has not yet made its way into current guidelines that still propose a "one size fits all" diagnostic approach. This thesis evaluated sex differences in coronary vascular disease to address the question whether this approach should be sex-specific instead.

\section{Main findings of this thesis}

In the first part of this thesis, we validated current symptom-based risk prediction scores for coronary vascular disease in women and men separately. Chapter $\mathbf{2}$ showed that a prediction model based on chest pain characteristics and risk factors for CAD performed well in both sexes, but that both the included predictors as well as the performance of the model changed when the model was developed in women and men separately. Chapter 3 illustrated that functional grading of complaints provides important information about mortality risk in women who consult the cardiologist with a variety of cardiac complaints.

In the second part, we showed that both the composition of the atherosclerotic plaque and the incidence of CAD are different in women and men. The literature review described in Chapter 4 showed that these differences are relevant in the evaluation of women and men at risk for cardiovascular disease who visit a cardiology clinic, as these differences in (patho)physiology imply use of a different approach to diagnose coronary vascular disease in women and men. Moreover, we found most literature comprised a domain of women and men at intermediate to high risk of CAD who were clinically referred for evaluation of a coronary artery stenosis. Little was 
known however on all, ranging from low- to high-risk, symptomatic women and men suspected of coronary vascular disease. The ARGUS study was introduced in Chapter 5 to study early-stage coronary vascular disease beyond obstructive CAD in all these symptomatic women and men. The aim of this study was to examine the prevalence of both macro and microvascular coronary vascular disease in all women and men visiting the outpatient clinic with chest pain or discomfort. The ARGUS study showed that women tend to have lower coronary calcium scores (CACS) when suffering from the same degree of CAD as men, pointing out that low CACS values might not be as reassuring in women as they are in men (Chapter 6 ). In addition, absolute CACS values were strongly related to all-cause mortality risk in both sexes despite overall lower values in women (Chapter 7).

In the third part we focused specifically on symptomatic women and men without obstructive CAD. Chapter 8 demonstrated that most of these patients remain symptomatic in the years following their diagnostic coronary angiography (CAG) appointment. In addition, they had a higher healthcare consumption than matched asymptomatic individuals that were not referred for CAG. Chapter 9 reviewed the value of non-invasive imaging tools to diagnose coronary microvascular dysfunction (CMD) in these symptomatic patients. It was highlighted that at this moment, despite the impressive developments of non-invasive imaging techniques for CMD, outcomes could not be compared due to heterogeneity in studies and it is not evident which modality or outcome measure to use.

Taken together, the three parts of this thesis demonstrate the importance of sex differences in various areas of coronary vascular disease. First, we showed that in women specific complaints and risk factors might have different implications for diagnosing CAD than in men and that the relation between these complaints and prognosis may differ. Second, the underlying pathophysiology might be different in women than in men, both within atherosclerotic plaques and during the disease trajectory. Third, the microvascular variant of coronary vascular disease that may occur more often in women is not benign and increases future healthcare use but often goes unrecognized due to lack of diagnostic tools.

These findings are not adequately reflected in current guidelines (2) that describe a similar diagnostic pathway for women and men based on one general risk prediction model with a focus on obstructive CAD. The individual predictors in these models are weighted by sex, yet there is otherwise little attention for sex differences in either the importance of specific symptoms and risk factors or the possibility that models might perform better when comprising different predictors in women and men. In 
addition, even though it mentions $\mathrm{CMD}$, the currently used guidelines largely ignore the non-obstructive variants of CAD that are more common in women due to lack of consistent data on risk factors and patient characteristics. In these, mostly female, patients with angina (equivalents) but non-obstructed coronaries, complaints are often caused by CMD or epicardial vasospasm $(3,4)$, although definite answers regarding sex differences in prevalence remain to be uncovered.

In the light of the evidence presented in this thesis, along with other supporting literature $(3,4)$, the "one size fits all" approach no longer seems suitable; nonobstructive CAD or functional coronary vascular disease should be equally included. Below, we will discuss the advantages and necessary steps that must be taken to develop sex-specific guidelines.

\section{What do we need to develop sex-specific guidelines...?}

To develop these guidelines new data is needed. Currently recommended and used risk prediction tools are developed, validated and periodically updated $(5,6)$ on quite old data (7) often collected from population-based cohort studies (8-10). These populations are incomparable with the symptomatic patients of today's practice for several reasons. First, research has shown that the atherosclerotic plaque is changing over time (11). Second, at the time these studies were conducted, we were not aware of the complete range of coronary vascular disease entities and how to identify these, thus only data on obstructive CAD and mortality was collected. Third, these population-based cohorts often include a relatively healthy population $(12,13)$, and do not represent the individuals visiting their general practitioner with complaints. Hence, we cannot continue to rely on risk prediction tools based on data originating from "historical" atherosclerotic tissue or studies focused on obstructive CAD and not designed to evaluate non-obstructive disease as well. To shape a diagnostic approach to identify (non-)obstructive variants of coronary vascular disease in symptomatic patients we need up-to-date data, reflecting the contemporary - risk factor profile and underlying pathologies of the - symptomatic patient population presenting at the doctor's office.

In addition to new data, also sex-stratified data is needed. For this purpose, it is important that women and men are both sufficiently represented in studies. Fortunately, at this moment many large trials are performed with a well-balanced dataset with equal numbers of women and men $(14,15)$. Thus, based on the large number of participants and the distribution of women and men, these studies will 
already be adequately powered to perform sex-stratified analyses. Hence, provision of sex-stratified data should not be a problem. Yet, at this moment sex-stratified analyses are not regularly performed or at least not documented (16). Mandated post-hoc analyses per sex would be a big step towards sex-sensitive guidelines and should be performed even when studies do not have enough power to really test for sex-differences. First, these estimates can then at least be included in large metaanalyses. Second, presenting these results will help to determine whether there are (no) differences between women and men that need to be appreciated. If all important studies from the past, especially those that are the foundation of our guidelines, would repeat their analysis per sex, powerful meta-analyses can be done, and this will help a lot to come closer to the sex-specific guidelines we need to adequately care for women and men. In designing new studies, a power calculation per sex could for example aid in determining the right number of women and men and prevent 'lack of power' limiting the provision of sex-specific findings afterwards. We suggest scientific journals to use their power to enforce performance and subsequent reporting of these analyses irrespective of whether differences are found between the sexes. The National Institutes of Health have led the way for the rest of the scientific community, as they mandate inclusion of sex in research as a biological variable (17). Similarly, the Dutch Heart Foundation plays an important role in funding of research focused on sex-differences and women's cardiovascular health.

Besides large trials, more and more data extracted from electronic health records and disease specific registries $(18,19)$ is becoming available that will aid in identification of relevant sex-differences and provide sex-sensitive data to reshape the guidelines in a sex-specific manner. For example, patients with angina and nonobstructed coronary arteries that undergo clinically indicated invasive coronary function testing are registered prospectively (19) for research purposes. By doing so, data will be systematically documented. Hence, naturally more data will become available that will be helpful in identifying specific complaints or risk profiles associated with these 'new' entities of coronary vascular disease, such as CMD or vasospasm. This will be an important first step in development of tools to predict the complete spectrum of coronary vascular disease. Yet, still only those patients with angina $(A)$ in whom obstructive CAD has been excluded, $(B)$ returning to their physician with sustained angina and $(C)$ with probably the most typical or suspect complaints will be referred for these coronary function tests by their cardiologist. Solely these patients will be included in the before-mentioned registry and provide us with structurally documented data. As a result, these registries do not comprise the less severe cases or patients with more atypical or vague complaints who might also suffer from CMD/vasospastic angina. 
Therefore, prospective cohort studies are still needed to fill the remaining knowledge gaps left behind by clinical care data. To gain insight in and develop a guideline that is applicable to the whole symptomatic population presenting at the outpatient clinic, research should comprise all these women and men in whom this guideline will be used. That is, symptomatic women and men consulting their clinician before referral for additional diagnostic testing. A prospective cohort study that assesses (all possible predictors for) coronary vascular disease in all symptomatic patients is required to exclude clinician's referral bias as well as study a new disease entity beyond the sparse information collected during regular care. Such a study will both provide an in-or exclusion diagnosis of coronary vascular disease as well as enable extensive phenotyping in all these symptomatic patients, which cannot be done regularly in clinical care. As an example of a study with a broad population ranging from low- to high-risk patients, in Chapter $\mathbf{5}$ we introduced the ARGUS study. In this study, all possibly relevant baseline data was prospectively collected: risk factors, complaints, biometric data, echocardiography, exercise testing and laboratory values. For outcome measures, next to coronary computed tomography angiography for anatomical evaluation of the coronaries, cardiac magnetic resonance imaging was included to also evaluate the presence or absence of myocardial ischemia. Recent research described promising results for use of non-invasive imaging modalities, such as cardiac magnetic resonance imaging, to provide information on CMD in a non-invasive manner (20-22). In theory, ARGUS might provide extensive baseline data and diagnostic information on both coronary artery stenosis and absolute perfusion of the myocardial blood flow. Unfortunately, even though quantification methods for absolute myocardial perfusion performed good in research (22-25), it seemed not yet possible to implement these techniques in the clinical care setting as we were unable to quantify myocardial perfusion to exclude or identify CMD. It will take time to take these techniques from the so-called bench to the bedside. Furthermore, the use of cardiac magnetic resonance imaging, or other non-invasive imaging modalities, does not fully exclude the presence of vasospasm or CMD. Hence, despite the current possibilities of cardiac magnetic resonance imaging, this might not be the way to go (yet) to in- or exclude coronary vascular disease completely. As the non-invasive modalities have not yet advanced enough to independently diagnose coronary dysfunction (Chapter 9), it can be imagined that a future prospective study would include the gold standard assessment for CMD, invasive coronary function testing in those with non-obstructive CAD. Yet, it might be difficult to motivate patients and even unethical to perform an extensive study such as the ARGUS study as there are non-negotiable risks accompanying the coronary function testing. So, for this part it might be necessary to do certain concessions, for example study specific high-risk patients first. Therefore, as suggested by the recent Dutch guidelines (26), it seems 
wise to start by studying patients in whom it is of importance or has treatment consequences, i.e., those with prolonged unexplained complaints or persistent suspicion of coronary dysfunction. Currently, ARGUS already has shown that it is (A) feasible to perform a prospective study in all women and men presenting at the outpatient clinic without referral bias and interference in the clinical care path and (B) collect all data needed to improve the diagnostic pathway for both women and men suspect of coronary vascular disease.

\section{... and what should these sex-specific guidelines say?}

To shape the future sex-sensitive guidelines in more detail, this thesis has already provided some directions. Based on the first part of this thesis, we can conclude that symptoms suspect of cardiovascular disease and commonly known cardiovascular risk factors possess value to diagnose CAD and predict mortality in both sexes. In Chapter 2 we showed, comparable to Bösner et al. (27), a high diagnostic value of chest pain characteristics and risk factors for CAD in both sexes. However, there are small sex differences in the specific characteristics and risk factors that support the diagnosis of CAD. To some extent, sex differences are already taken into account in the current guidelines (2). For example, the pre-test probability for obstructive CAD is calculated in the light of sex and performance of these calculations is good in both women and men (7). Yet, as our results highlight relevant differences in both the included predictors as well as the strength of chest pain characteristics and risk factors for CAD when models were developed per sex (Chapter 2), it might be worthwhile to re-fit the current models and assess the included predictors for each sex separately. In other words, sex-specifically developed models with sex-specifically selected predictors seem preferred over currently used universal-models fitted with sex included as a covariate, especially in women. This does not necessarily imply that these predictors will be female-specific factors, as previous research showed that inclusion of pregnancy hypertension did not improve currently used cardiovascular risk prediction tools (28), yet a sex-specific set of universal cardiovascular risk factors might be more accurate for prediction of CAD as compared to an identical set of factors weighted per sex. Based on our results, in women the model would for example also include dyspnea whereas in men functional grading of complaints might be included (Chapter 2).

These renewed symptom-based models will mainly be of relevance in women, as most tools used nowadays are developed in predominantly male populations and therefore already perform very well in men. Even though we did not study this, and 
it is beyond the scope of this piece, sex-sensitive re-appreciation is very likely to be also fruitful for other tools used in risk prediction such as blood-based biomarkers. To illustrate, an unrecognized myocardial infarction is more common in women (29). A recent study showed that use of sex-specific thresholds for troponins might identify more women with myocardial injury as compared to the currently used universal thresholds (30) which might reduce the incidence of unrecognized myocardial infarctions in women. For our proposed sex-specific guidelines, validation and, if required, improvement of all tools previously developed in male-biased datasets should thus be performed in the light of sex with use of balanced datasets including all coronary vascular disease entities.

Conjointly with re-appreciation of the value of complaints per sex, these guidelines should also consider sex when choosing the most optimal diagnostic approach. As the variants of coronary vascular disease can clearly be different between women and men, it does not make sense to simply apply an identical diagnostic path to both women and men. As emphasized extensively in the previous paragraphs, the diagnostic approach should not just include obstructive CAD but also non-obstructive variants of coronary vascular disease or other cardiac diseases that can present similarly with chest pain or discomfort such as heart failure. Thus, we stress the need to shape the diagnostic pathway with the most probable underlying pathologies in mind. This could be an initial, but not solely, functional approach in women and a more anatomical approach in men.

The exact roadmap of this sex-specific diagnostic approach beyond obstructive CAD remains largely to be determined. At this moment we lack diagnostic models for non-obstructive types of coronary vascular disease as most cardiovascular (imaging) studies in both sexes concentrated on diagnosing or excluding obstructive CAD in patients at intermediate to high risk (i.e., patients referred for clinically indicated additional testing $(31,32))$. Hence, as there is a lack of knowledge on clinical entities of coronary vascular disease beyond "classical" obstructive CAD caused by calcified atherosclerotic plaques, current models are only capable of diagnosing obstructive CAD. The recently developed guideline 'Chest Pain With Non-obstructed Coronary Arteries' from the Dutch Society of Cardiology (26) provides some guidance in the assessment of patients without obstructive CAD, by advising coronary functional testing to assess CMD and vasospasm. Nonetheless, to date, this guideline is unable to provide clear recommendations regarding risk factors or symptoms that can be used to determine in which patients coronary function should indeed be assessed. However, if our suggested steps will be carried out (mandatory (post-hoc) sex-stratified analyses, use of available clinical care data and prospective trials to fulfill the 
remaining knowledge gaps) we are hopeful. Consistent data on risk factors, patient characteristics and diagnostic information on the presence or absence of coronary vascular disease will then become available to provide more guidance in the future. Based on these data separate coronary vascular disease phenotypes can be identified and used to create the perfect roadmap for the diagnostic approach.

Besides lack of data from studies performed in the "obstructive-CAD" era, development of models focused on non-obstructive variants of coronary vascular disease is further complicated by the fact that no diagnostic tool is capable of direct visualization of the coronary microvasculature. Thus, patients need to be exposed to invasive coronary angiography to enable coronary function testing to receive a diagnosis, which hinders large scale research due to its invasive nature. The Coronary Vasomotor Dysfunction (COVADIS) study group has recently provided recommendations on when and how coronary vascular dysfunction should be assessed in patients with angina and no obstructive coronary artery disease (33) and showed that it is feasible in clinical practice (34). Furthermore, they reported that medical therapy stratified based on coronary function testing significantly improves symptoms and quality of life. These results indicate that coronary function testing is of added value as compared to medical therapy alone without invasive testing to confirm a diagnosis. Hence, it is likely that this technique will conquer a place in the care path of women and men with chest pain and non-obstructed coronaries. However, even though the approach as suggested by the COVADIS group seems feasible and fruitful, invasive testing puts a burden on patients and does not come without risks. Luckily, several non-invasive imaging techniques to diagnose CMD are currently being developed and evaluated (Chapter 9). Alas, despite the impressive developments of non-invasive imaging techniques to diagnose CMD, due to heterogeneity in methodology and reporting of outcome measures in current studies translation to clinical practice is still hampered. To live up to the promise of non-invasive imaging to diagnose CMD, large, well-designed studies are needed including a clearly defined group of patients, preferably those with objectified signs of ischemia and non-obstructive CAD as determined by gold-standard anatomical assessment. Furthermore, standardization of outcome parameters per imaging modality is warranted to provide clinically applicable, possibly sex-specific, reference values for the diagnosis of CMD in the future. The most promising modality is not evident yet but should at least be determined based on local expertise and availability. Even more important, it should be chosen in the light of the most probable disease in the individual male or female patient. For example, the second part of this thesis underlines the importance of a sex-specific view on coronary vascular disease and evaluation beyond coronary calcification for optimal risk prediction, especially in women. Thus, despite its moderately good performance, especially in women relying on the coronary calcium score only might not be the way to go. 
Until the diagnostic pathway and treatment for these symptomatic women and men with non-obstructed coronaries will be optimal, close attention is warranted as we now know they might suffer from $C M D$, and thus put a large burden on health care (35), despite their "normal" CAG. Moreover, we showed after a CAG result of nonobstructed coronary arteries $(A)$ in both sexes, the majority of women and men remain symptomatic and (B) they frequently consult their GP with cardiovascular and psychological issues (Chapter 9). One can argue how, when and by whom these patients should be followed up. Since we observed that almost all of them visit their general practitioner (GP) more often, we suggest there is an important role for the GPs to seize this problem. It might pay off to implement regular follow-up visits after a "normal" CAG to evaluate whether these patients suffer from prolonged complaints or follow up their cardiac function. The importance of follow-up of these individuals is even more emphasized by the fact that we observed that both women and men with non-obstructed coronary arteries consume evidently more care for heart failure in the years following CAG than matched asymptomatic individuals. This indicates that these symptomatic patients, despite their non-obstructed coronaries, might be at risk for development of heart failure. Our results point out that, by focusing on coronary macrovascular disease only, we fail these patients and possibly miss the opportunity to prevent heart failure. This is an unwanted situation for both the patients and the healthcare system, as the medical burden and costs associated with heart failure are high, especially when compared to regular GP visits and follow-up. Hence, the high number of GP consultations for both cardiovascular and psychosocial care stress the need to monitor symptomatic women and men after a "normal" CAG beyond MACE not in the least because quality of life influences the prevalence of cardiovascular events and mortality (36). These "soft" outcomes might point us towards a burden in these patients that is currently missed by our focus on "hard" endpoints such as mortality. Along these lines, the upcoming research on patient reported outcome measures is a promising development in cardiology (37).

\section{Future perspectives: call for a sex-specific view}

In conclusion, this thesis has demonstrated that there is still an undisputed need for a sex-specific view on patients suspect of coronary vascular disease, as differences in coronary vascular disease between women and men are evident and extensive. Thus, the need for sex-stratified cardiovascular research and subsequently derived evidencebased medicine is clear. It is of paramount importance to state that, of course, personalized medicine should go further than just sex. As discussed in Chapter $\mathbf{7}$, also ethnicity is an important factor to keep in mind when evaluating coronary vascular 
disease on a more personalized level. Nonetheless, sex is an indisputable variable we emphasize in this thesis, as women comprise at least halve of the of the world's population, probably even the majority due to the lower life expectancy in men, and above the age of 55 there are considerably more women as compared to men (38). Thus, sex is a vital and easily appreciated factor that we at least can consider from now on in research to come one step closer to inclusive individualized precision medicine.

We feel we have provided some guidance in how this guideline could be crafted and argue that we should not compare the sexes one on one but appreciate each sex separately. Both obstructive, macrovascular disease and non-obstructive or microvascular disease occur in women and in men. We should not think in blue and pink. "Men suffer from obstructive CAD" and "women have CMD" does not do justice to either sex. We showed that even though non-obstructive CAD is relatively more common in women, the absolute numbers are equally high in men. Also, even though obstructive CAD is more common in men, the prevalence and impact in women cannot be overlooked, especially since prognosis of women suffering from severe obstructive CAD is worse as compared to men (39). In other words, there is no such thing as a woman-only or man-only disease. As such, it does not appear to be wise to study just one sex nor to use universal guidelines. A clinician should evaluate his or her patients through sex-colored glasses, supported by a specific guideline for each sex. Evidence based medicine for women should be based on evidence in women and vice versa for men.

To meet the need for sex-specific guidelines, we propose to follow-up on a sex-specific diagnostic approach. We envision to accomplish this via a two-track policy. We recommend to firstly re-analyze currently available data per sex and secondly craft prospective cohort studies in the patient domain of interest to fill remaining knowledge gaps on differences between women and men. Via these two main routes, similarities as well as differences between women and men will become clear and sufficient data will be available to develop sex-specific guidelines that appreciate both women and men as they are: equal but not identical. Based on these guidelines we will be able to provide women and men with the diagnostic testing and therapy they need, instead of performing all testing in all patients. This will decrease the need for repeat and multiple testing, provide tailored treatment strategies and substantially lower the burden on both individual patients as the healthcare system. An evidencebased, sex-specific clinical workflow will make future healthcare sustainable and optimize care for individual patients. The body of evidence provided in this thesis leaves the necessity for appreciation of women and men separately in the doctor's office undisputed and calls for action. 


\section{REFERENCES}

1. Mensah GA, Roth GA, Fuster V. The global burden of cardiovascular diseases and risk factors: 2020 and beyond. American College of Cardiology Foundation Washington, DC; 2019.

2. Knuuti J, Wijns W, Saraste A, Capodanno D, Barbato E, Funck-Brentano C, et al. 2019 ESC Guidelines for the diagnosis and management of chronic coronary syndromes: the Task Force for the diagnosis and management of chronic coronary syndromes of the European Society of Cardiology (ESC). European heart journal. 2020;41(3):407-77.

3. Kaski J-C, Crea F, Gersh BJ, Camici PG. Reappraisal of ischemic heart disease: fundamental role of coronary microvascular dysfunction in the pathogenesis of angina pectoris. Circulation. 2018;138(14):1463-80.

4. Ong P, Athanasiadis A, Borgulya G, Mahrholdt H, Kaski JC, Sechtem U. High prevalence of a pathological response to acetylcholine testing in patients with stable angina pectoris and unobstructed coronary arteries: the ACOVA Study (Abnormal COronary VAsomotion in patients with stable angina and unobstructed coronary arteries). Journal of the American College of Cardiology. 2012;59(7):655-62.

5. Achenbach S, Aleksandrova K, Amiano P, Sebastian D-S, Amouyel P, Andersson J, et al. SCORE2 risk prediction algorithms: new models to estimate 10 -year risk of cardiovascular disease in Europe. European Heart Journal.

6. Genders TS, Steyerberg EW, Hunink MM, Nieman K, Galema TW, Mollet NR, et al. Prediction model to estimate presence of coronary artery disease: retrospective pooled analysis of existing cohorts. Bmj. 2012;344.

7. Bing R, Singh T, Dweck MR, Mills NL, Williams MC, Adamson PD, et al. Validation of European Society of Cardiology pre-test probabilities for obstructive coronary artery disease in suspected stable angina. European Heart JournalQuality of Care and Clinical Outcomes. 2020;6(4):293-300.

8. Conroy RM, Pyörälä K, Fitzgerald Ae, Sans S, Menotti A, De Backer G, et al. Estimation of ten-year risk of fatal cardiovascular disease in Europe: the SCORE project. European heart journal. 2003;24(11):987-1003.

9. Mahmooda SS, Levy D, Vasan R, Wang T. The Framingham Heart Study and the epidemiology of cardiovascular diseases: A historical perspective. Lancet. 2014;383(9921):999-1008.

10. Wilson PW, D’Agostino RB, Levy D, Belanger AM, Silbershatz H, Kannel WB. Prediction of coronary heart disease using risk factor categories. Circulation. 1998;97(18):1837-47.

11. van Lammeren GW, den Ruijter HM, Vrijenhoek JE, van der Laan SW, Velema E, de Vries J-PP, et al. Timedependent changes in atherosclerotic plaque composition in patients undergoing carotid surgery. Circulation. 2014;129(22):2269-76.

12. Tabrizi R. Untersuchungen im Hinblick auf Lebensqualität und seelische Gesundheit bei Patienten mit malignem Melanom in Abhängigkeit von tumorspezifischen Parametern. 2020.

13. Fry A, Littlejohns TJ, Sudlow C, Doherty N, Adamska L, Sprosen T, et al. Comparison of sociodemographic and health-related characteristics of UK Biobank participants with those of the general population. American journal of epidemiology. 2017;186(9):1026-34.

14. Ford TJ, Corcoran D, Oldroyd KG, McEntegart M, Rocchiccioli P, Watkins S, et al. Rationale and design of the British Heart Foundation (BHF) Coronary Microvascular Angina (CorMicA) stratified medicine clinical trial. American heart journal. 2018;201:86-94.

15. Hoffmann U, Ferencik M, Udelson JE, Picard MH, Truong QA, Patel MR, et al. Prognostic value of noninvasive cardiovascular testing in patients with stable chest pain: insights from the PROMISE trial (Prospective Multicenter Imaging Study for Evaluation of Chest Pain). Circulation. 2017;135(24):2320-32.

16. Bots SH, Groepenhoff F, Eikendal AL, Tannenbaum C, Rochon PA, Regitz-Zagrosek V, et al. Adverse drug reactions to guideline-recommended heart failure drugs in women: a systematic review of the literature. JACC: Heart Failure. 2019;7(3):258-66.

17. Arnegard ME, Whitten LA, Hunter C, Clayton JA. Sex as a biological variable: a 5-year progress report and call to action. Journal of Women's Health. 2020;29(6):858-64.

18. Komajda M, Cosentino F, Ferrari R, Kerneis M, Kosmachova E, Laroche $C$, et al. Profile and treatment of chronic coronary syndromes in European Society of Cardiology member countries: The ESC EORP CICD-LT registry. European journal of preventive cardiology. 2021;28(4):432-45.

19. Konst RE, Damman P, Pellegrini D, Hartzema-Meijer MJ, van Uden BJ, Jansen TP, et al. Vasomotor dysfunction in patients with angina and nonobstructive coronary artery disease is dominated by vasospasm. International Journal of Cardiology. 2021;333:14-20.

20. Wöhrle J, Nusser T, Merkle N, Kestler HA, Grebe OC, Marx N, et al. Myocardial perfusion reserve in cardiovascular magnetic resonance: correlation to coronary microvascular dysfunction. Journal of Cardiovascular Magnetic Resonance. 2006;8(6):781-7. 
21. Zhou W, Lee JCY, Leung ST, Lai A, Lee T-F, Chiang JB, et al. Long-term prognosis of patients with coronary microvascular disease using stress perfusion cardiac magnetic resonance. JACC: Cardiovascular Imaging. 2021;14(3):60211.

22. Thomson LE, Wei J, Agarwal M, Haft-Baradaran A, Shufelt C, Mehta PK, et al. Cardiac magnetic resonance myocardial perfusion reserve index is reduced in women with coronary microvascular dysfunction: a National Heart, Lung, and Blood Institute-sponsored study from the Women's Ischemia Syndrome Evaluation. Circulation: Cardiovascular Imaging. 2015;8(4):e002481.

23. Karamitsos TD, Jerosch-Herold M, Pegg TJ, Cheng AS, Arnold JR, Neubauer S, et al., editors. 2011 Quantitative cardiac magnetic resonance perfusion imaging at 3 Tesla in patients with suspected coronary artery disease. Journal of Cardiovascular Magnetic Resonance; 2008: BioMed Central.

24. Feher A, Sinusas AJ. Quantitative assessment of coronary microvascular function: dynamic single-photon emission computed tomography, positron emission tomography, ultrasound, computed tomography, and magnetic resonance imaging. Circulation: Cardiovascular Imaging. 2017;10(8):e006427.

25. Nagel E, Klein C, Paetsch I, Hettwer S, Schnackenburg B, Wegscheider K, et al. Magnetic resonance perfusion measurements for the noninvasive detection of coronary artery disease. Circulation. 2003;108(4):432-7.

26. NVvC GW. NVVC Leidraad "Pijn op de borst zonder obstructief coronairlijden. 2020.

27. Bösner S, Becker A, Haasenritter J, Abu Hani M, Keller H, Sönnichsen AC, et al. Chest pain in primary care: epidemiology and pre-work-up probabilities. The European journal of general practice. 2009;15(3):141-6.

28. Dam V, Onland-Moret NC, Verschuren WM, Boer JM, Benschop L, Franx A, et al. Cardiovascular risk model performance in women with and without hypertensive disorders of pregnancy. Heart. 2019;105(4):330-6.

29. van der Ende MY, Juarez-Orozco LE, Waardenburg I, Lipsic E, Schurer RA, van der Werf HW, et al. Sex-Based Differences in Unrecognized Myocardial Infarction. Journal of the American Heart Association. 2020;9(13):e015519.

30. Lee KK, Ferry AV, Anand A, Strachan FE, Chapman AR, Kimenai DM, et al. Sex-specific thresholds of high-sensitivity troponin in patients with suspected acute coronary syndrome. Journal of the American College of Cardiology. 2019;74(16):2032-43.

31. Pagidipati N, Hill L, Coles A, Udelson JE, Picard MH, Pellikka PA, et al. Implications of Inconclusive Noninvasive Test Results in Stable Patients With Suspected Coronary Artery Disease: Insights From the PROMISE Trial. Circulation. 2017;136(suppl_1):A18647-A.

32. The SCOT-HEART investigators. CT coronary angiography in patients with suspected angina due to coronary heart disease (SCOT-HEART): an open-label, parallel-group, multicentre trial. The Lancet. 2015;385(9985):2383-91.

33. Ford TJ, Ong P, Sechtem U, Beltrame J, Camici PG, Crea F, et al. Assessment of vascular dysfunction in patients without obstructive coronary artery disease: why, how, and when. Cardiovascular Interventions. 2020;13(16):1847-64.

34. Ford TJ, Stanley B, Good R, Rocchiccioli P, McEntegart M, Watkins S, et al. Stratified medical therapy using invasive coronary function testing in angina: the CorMicA trial. Journal of the American College of Cardiology. 2018;72(23 Part A):2841-55.

35. Shaw LJ, Merz CB, Pepine CJ, Reis SE, Bittner V, Kip KE, et al. The economic burden of angina in women with suspected ischemic heart disease. Circulation. 2006;114(9):894-904.

36. Gohar A, Gijsberts CM, Haitjema S, Pasterkamp G, de Kleijn DP, Asselbergs FW, et al. Health-related quality of life and outcome in atherosclerosis-Does sex matter? International journal of cardiology. 2016;212:303-6.

37. Greenhalgh J. The applications of PROs in clinical practice: what are they, do they work, and why? Quality of Life Research. 2009;18(1):115-23.

38. ClA. Factbook.

39. Gijsberts CM, Santema BT, Asselbergs FW, de Kleijn DP, Voskuil M, Agostoni P, et al. Women undergoing coronary angiography for myocardial infarction or who present with multivessel disease have a poorer prognosis than men. Angiology. 2016;67(6):571-81. 

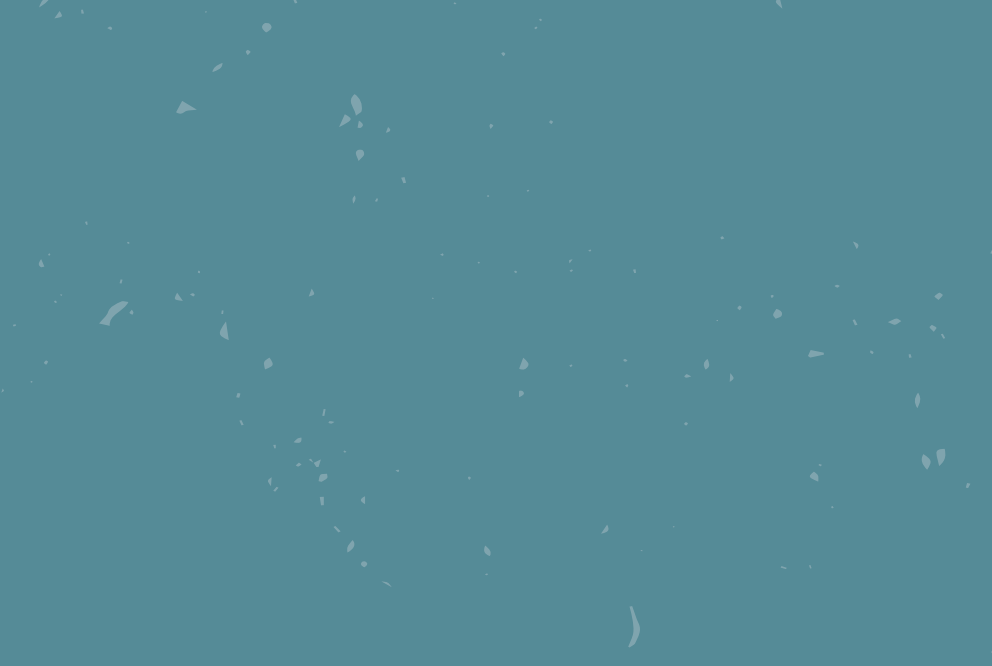

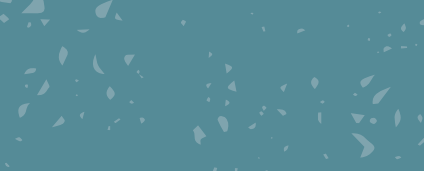




\section{APPENDIX}

Dankwoord

\section{Samenvatting in het Nederlands :}

\section{List of publications \\ Curriculum Vitae}
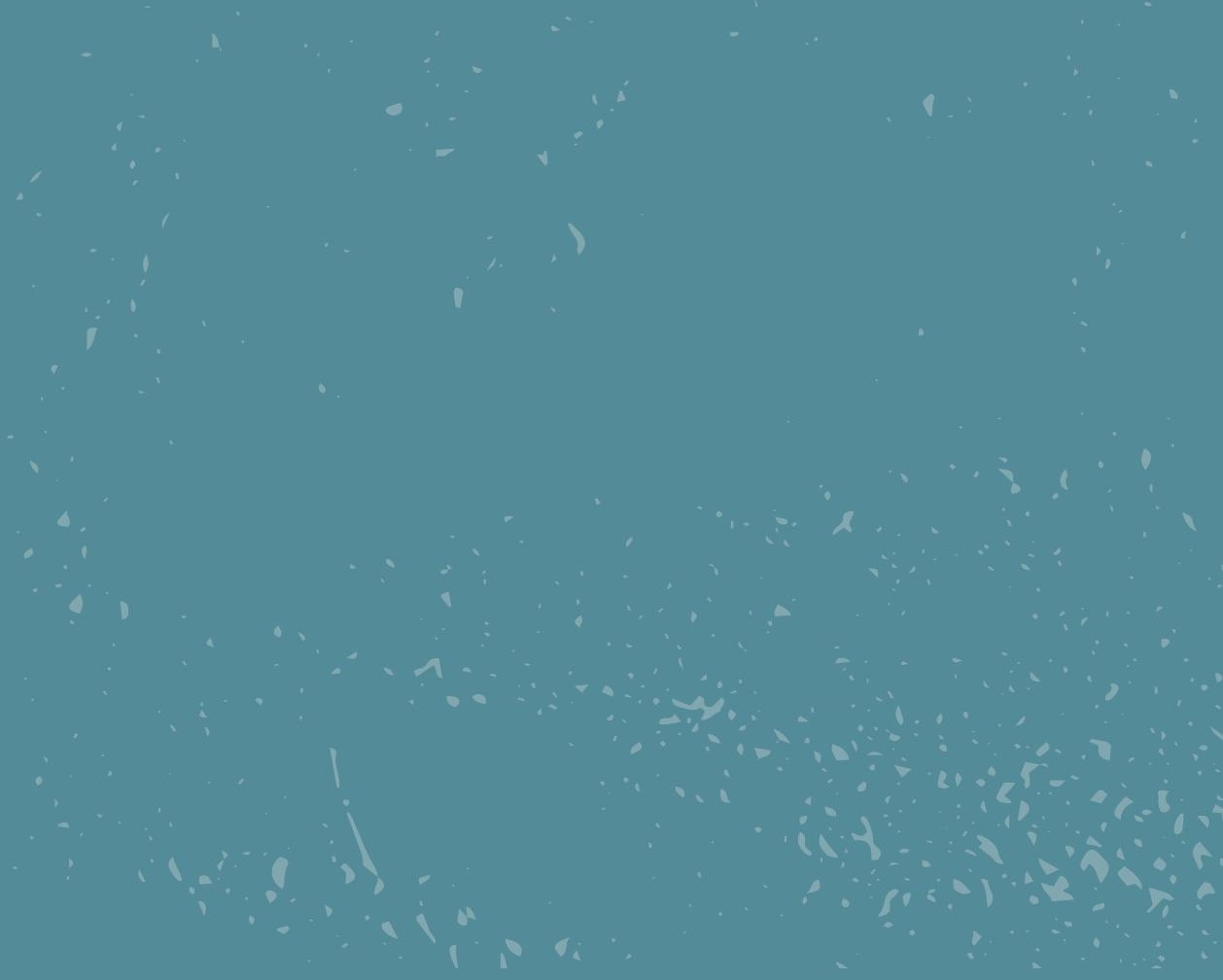


\section{Samenvatting in het Nederlands}

\section{Achtergrond}

Hart- en vaatziekten zijn wereldwijd nog steeds de belangrijkste doodsoorzaak bij mannen en bij vrouwen. Hart- en vaatziekten zijn vaak het gevolg van slagaderverkalking, ook wel atherosclerose genoemd. Bij atherosclerose vormt zich een plaque; een plaatselijke verdikking in de wand van een bloedvat die ontstaat als gevolg van een ontstekingsreactie. Een plaque zorgt ervoor dat de opening van het bloedvat vernauwd en verstijfd raakt en er minder bloed door het bloedvat kan stromen. Zoals de veel gebruikte term slagaderverkalking al aangeeft, bevat een plaque vaak kalk. Echter, een plaque kan ook gemaakt zijn van zacht weefsel en weinig tot geen kalk bevatten. Dan spreekt men van een niet-verkalkte of niet-gecalcificeerde plaque.

Bij coronairlijden is sprake van atherosclerose van de bloedvaten van het hart. Het bloedvatsysteem van het hart bestaat uit drie grote bloedvaten, ook wel coronairen of kransslagaders genoemd. Deze grote bloedvaten vertakken zich vervolgens verder tot in de kleinste haarvaten (de microcirculatie of microvasculatuur). Deze bloedvaten zorgen ervoor dat het aanbod van bloed, waarin zuurstof en voedingsstoffen worden aangeboden aan de hartspier, aansluit bij de vraag (oftewel de behoefte). Dit vaatsysteem van het hart is gevoelig voor atherosclerose. Als een van deze bloedvaten dusdanig vernauwd raakt dat de bloedtoevoer zo ernstig wordt verminderd dat het hart onvoldoende zuurstof krijgt, kunnen er klachten ontstaan. De meest bekende klacht is pijn op de borst. Bij een beperkte vernauwing van het bloedvat treden deze klachten met name op bij inspanning, wanneer het hart meer zuurstof nodig heeft. In rust verdwijnen deze klachten dan ook weer. In dit geval is er sprake van stabiel coronairlijden. Als de mate van vernauwing toeneemt, doordat de plaque steeds groter wordt, kunnen er ook klachten optreden in rust. Men spreekt dan van instabiel coronairlijden.

Coronairlijden kan leiden tot een acute hartstilstand wanneer de bloedtoevoer naar de hartspier plots (bijna) volledig wordt onderbroken. Deze onderbreking treedt op wanneer een bloedstolsel dat zich boven op de plaque vormt acuut het bloedvat verstopt. Zo'n bloedprop heet een trombus. Sinds de jaren '80 weten we dat er twee oorzaken te onderscheiden zijn waardoor een trombus ontstaat; bij de ene vorm, een 'plaque ruptuur', scheurt de plaque open. Vervolgens komt de inhoud van de plaque, o.a. bestaande uit ontstekingscellen, vrij en vormt zich een trombus boven op deze 'wond'. Bij de andere vorm, 'plaque erosie', blijft de plaque zelf intact maar ontstaat een soort 'schaafwond' met daarbovenop een trombus. We zien een plaque ruptuur vaker bij mannen. Plaque erosie komt vaker voor bij vrouwen. 
Onderzoek naar hart- en vaatziekten was in het verleden vooral gericht op mannen. Om deze reden weten we het meeste van vormen van hart- en vaatziekten die klassiek veel bij mannen voorkomen, zoals een plaque ruptuur en verkalkte plaques. Inmiddels wordt de invloed van geslacht op hart- en vaatziekten steeds meer op waarde geschat. Geslachtsverschillen in hart- en vaatziekten in het algemeen, en meer specifiek in (het ontwikkelen van) plaques, worden de laatste jaren steeds meer beschreven. Ondanks de toename in aandacht voor, en kennis over, man-vrouw verschillen in hart- en vaatziekten, loopt de cardiologische praktijk nog wel achter. Men beoordeelt en behandelt patiënten in de hedendaagse praktijk namelijk vooral op basis van 'mannelijk' wetenschappelijk bewijs. Daardoor is de huidige 'evidence-basedmedicine' nog steeds voornamelijk op mannen gebaseerd. Naast onvoldoende kennis over het vrouwhart, speelt het feit dat we vooral veel geleerd hebben over de opbouw van plaques bij patiënten met een vergevorderde vorm van atherosclerose ook een rol bij de lacune in kennis. Daardoor weten we namelijk nog heel weinig over plaque vorming bij mannen en vrouwen in een vroeger stadium. Dit komt omdat het meeste onderzoek gebaseerd is op het door de microscoop bekijken van plaques. Plaques moeten uit het lichaam gehaald worden voordat we ze op deze manier door de microscoop kunnen bekijken. Dit betekent dat plaques hiervoor alleen beschikbaar zijn als ze (1) tijdens een operatie verwijderd worden of (2) als iemand reeds overleden is. Oftewel, alleen plaques van mannen en vrouwen met een vergevorderde vorm van vaatlijden (die hiervoor geopereerd worden of overlijden) kunnen door de microscoop bekeken worden. Om deze hiaten in de hedendaagse kennis op te vullen, of in ieder geval te verkleinen, is onderzoek nodig naar mannen én vrouwen vroeg in het ziektebeloop van slagaderverkalking.

\section{DEEL I - Klachten}

In het eerste deel van dit proefschrift wordt een eerste stap gezet om kennis over mannen en vrouwen in een vroeg stadium van ziekte te vergroten. Dit hebben we gedaan door te onderzoeken in hoeverre lichamelijke klachten die patiënten vermelden tijdens een eerste consult bij een cardioloog gebruikt kunnen worden om risico op ziekte en overlijden te voorspellen. Dit hebben we apart voor vrouwen en mannen bekeken. Hiervoor hebben we in hoofdstuk 2 en $\mathbf{3}$ mannen en vrouwen onderzocht die na verwijzing door de huisarts de cardioloog hebben bezocht met klachten die verdacht zijn voor, oftewel die zouden kunnen wijzen op, hart- en vaatziekten. Bij de cardioloog volgt een uitgebreide evaluatie waarbij onder andere de bloeddruk wordt gemeten, een fietstest wordt gedaan en een hartfilmpje wordt gemaakt. Vervolgens vraagt de cardioloog de klachten in detail uit, spreekt eventueel 
aanvullend onderzoek af en/of stelt een diagnose. Deze informatie wordt vervolgens opgeslagen in het elektronisch patiëntendossier. Om te onderzoeken in hoeverre klachten gebruikt kunnen worden om ziekte (hoofdstuk 2) en/of risico op overlijden (hoofdstuk 3) te voorspellen, hebben we deze informatie uit het elektronisch patiëntendossier gebruikt. Hierbij vonden we dat klachten van waarde zijn voor het stellen van de diagnose 'coronairlijden' en voor het voorspellen van sterfte. Dezelfde voorspellende modellen werkten goed bij zowel mannen als vrouwen. Maar wat opviel was dat hoe sterk elke specifieke klacht bijdraagt aan deze voorspelling verschilde tussen mannen en vrouwen. Dit betekent dat voorspellende modellen die apart voor mannen en vrouwen worden ontwikkeld het risico nóg beter zullen kunnen inschatten. Deze kennis is vooral van belang voor vrouwen, omdat de huidige voorspelmodellen vooral in en voor mannen zijn ontwikkeld en in mannen dus al goed werken.

\section{DEEL II - Onderliggend ziekteproces en beeldvorming}

Het tweede deel van dit proefschrift geeft een overzicht van man-vrouw verschillen in het ziektebeeld coronairlijden. Eerst wordt in hoofdstuk 4 een overzicht gegeven van de tot op heden beschikbare literatuur die deze man-vrouw verschillen beschrijft. Dit betreft zowel de onderliggende biologische verschillen in coronairlijden, alsook de mogelijkheden die er zijn om coronairlijden in beeld te brengen. Uit dit literatuuronderzoek kwam onder andere naar voren kwam dat onderzoek naar coronairlijden tot nu toe vooral gebaseerd was op patiënten die na bezoek aan de cardioloog verwezen werden voor aanvullend onderzoek naar coronairlijden. Er is nog weinig bekend is over alle mannen en vrouwen die de polikliniek cardiologie bezoeken maar (nog) niet worden doorverwezen voor aanvullend onderzoek. Hierdoor mis je dus de mannen en vrouwen met een lager risico, of minder verdachte klachten, die niet worden verwezen voor aanvullend onderzoek. Om inzicht te krijgen in al deze mannen en vrouwen met klachten in een vroeg stadium, nl. een eerste bezoek aan de cardioloog i.v.m. klachten, is de ARGUS studie gestart. Deze studie wordt beschreven in hoofdstuk 5 . Het doel van deze studie is om in kaart te brengen hoe vaak er coronairlijden voorkomt bij mannen en vrouwen die voor het eerst de cardioloog bezoeken met pijn op de borst klachten. Hierbij wordt zowel gekeken naar de grote kransslagaders (de macrocirculatie) als de kleine haarvaten (de microcirculatie) die tot in de hartspier doorlopen. Om beide vormen van coronairlijden te onderzoeken worden er in deze studie twee beeldvormende technieken gebruikt om het hart en haar doorbloeding in beeld te brengen: een CT- en MRI-scan. Met de CT wordt gekeken naar plaquevorming in de grote kransslagaders. De MRI wordt 
vervolgens gemaakt om te kijken naar de doorbloeding tot in de kleinste vaatjes van de hartspier.

Met behulp van de resultaten van de ARGUS studie is in hoofdstuk $\mathbf{6}$ gekeken naar man-vrouw verschillen in coronairlijden op de CT-scan. Tijdens de CT-scan wordt de kalkscore bepaald. Deze score beschrijft de hoeveelheid kalk in de kransslagaders. Een hoge kalkscore is een belangrijke voorspeller voor hart- en vaatziekte en sterfte. Ook is het met behulp van de CT-scan mogelijk om de mate van (niet)-verkalkte plaquevorming in de kransslagaders te bekijken. De relatie tussen twee metingen op de CT-scan hebben we vergeleken: de kalkscore en een nieuwe maat voor de ernst van coronairlijden, de zogenaamde CAD-RADS score. De CAD-RADS score geeft de hoeveelheid plaque in de kransslagaders aan en hoeveel procent vernauwing van de kransslagaders deze plaques geven. Uit ons onderzoek bleek dat de kalkscore sterk samenhangt met deze CAD-RADS score. Oftewel, bij een ernstigere mate van coronairlijden (hogere CAD-RADS score), neemt de hoeveelheid kalk in de kransslagaders toe. Wat echter duidelijk opviel was dat de stijging van de kalkscore bij toename van de vernauwing minder groot was in vrouwen dan in mannen. Oftewel, vrouwen hebben lagere kalkscores dan mannen bij eenzelfde ernst van coronairlijden. Deze resultaten geven aan dat een lage kalkscore mogelijk minder geruststellend is bij vrouwen dan bij mannen. Ook wijzen deze resultaten erop dat onderzoek naar CT-uitkomsten per geslacht uitgevoerd moet worden om de waarde van deze parameters binnen mannen en vrouwen te bepalen. Vervolgens hebben we in hoofdstuk 7 onderzocht of kalkscore in mannen en vrouwen die de cardioloog bezoeken een goede voorspeller is voor sterfte. Daarnaast hebben we bekeken of de absolute kalkscore of een gepersonaliseerde waarde voor de hoeveelheid kalk op basis van leeftijd, geslacht en afkomst beter gebruikt kan worden voor het voorspellen van sterfte. De absolute kalkscore bleek sterk voorspellend voor het risico op sterfte bij zowel mannen als vrouwen die de cardioloog bezoeken. Opvallend was dat, ook al was de kalkscore over het algemeen behoorlijk wat lager in vrouwen dan in mannen, de absolute kalkscore het risico op sterfte minstens net zo goed voorspelde als een gepersonaliseerde waarde. Deze resultaten komen overeen met resultaten uit een eerder onderzoek dat uitgevoerd is in gezonde mannen en vrouwen zonder klachten. Naast de voorspellende waarde van kalk, is ook gekeken naar de rol die de mate van vaatvernauwing speelt in de relatie tussen kalkscore en sterfte. Bij vrouwen kan sterfte mogelijk beter voorspeld worden als de kalkscore én de mate van vernauwing samen worden bekeken, maar bij mannen was dit niet het geval. Dit zou verklaard kunnen worden doordat vrouwen vaker niet-verkalkte plaques hebben. Mogelijk zorgen deze niet-verkalkte plaques voor vernauwingen die, boven op de kalkscore, bijdragen aan een verhoogd risico op sterfte. Deze niet-verkalkte plaques mis je als je alleen kijkt 
naar de kalkscore om het risico op sterfte in te schatten. Concluderend is in dit tweede deel van dit proefschrift bewijs aangedragen dat het tijd is om de waarde van beeldvorming parameters per geslacht te bekijken bij mannen en vrouwen met klachten die verdacht zijn voor coronairlijden.

\section{Deel III - Coronairlijden zonder vernauwde kransslagaders}

Naast man-vrouw verschillen in het voorkomen van coronairlijden met vernauwingen in de kransslagaders, is ook steeds meer aandacht voor mannen en vrouwen met klachten passend bij zuurstoftekort van het hart waarbij géén vernauwing wordt gevonden in de grote vaten van het hart. Het laatste deel van dit proefschrift richt zich om deze reden op een tot nu toe onderbelichte groep met een hoger risico op eerder overlijden aan hart- en vaatziekten; mannen en vrouwen met klachten passend bij zuurstoftekort van het hart zonder vernauwde kransslagaders. Vermoed wordt, dat de oorzaak van klachten en hart- en vaatziekten bij deze patiënten gelegen is in een verstoring van de functie van de microcirculatie van het hart, dat wil zeggen de eerdergenoemde kleine(re) haarvaatjes van het hart. Eerder onderzoek heeft laten zien dat deze patiënten een hoger risico hebben op hartinfarcten, beroertes en overlijden. Maar er is nog weinig bekend over de totale ziektelast van deze patiënten die, ondanks beangstigende pijn op de borst klachten, naar huis worden gestuurd omdat er geen oorzaak gevonden wordt. Naast een hoger risico op deze harde eindpunten, hebben deze mannen en vrouwen vaak nog langdurige (hart)klachten. Om inzicht te krijgen in de grootte van deze last, hebben wij in hoofdstuk 8 onderzoek gedaan naar de zorgbehoefte voor hart- en vaatziekten en psychische problemen van mannen en vrouwen die geen vernauwingen van de kransslagaders bleken te hebben bij hartkatheterisatie. Hierbij bleek, net als eerder al was beschreven, dat tijdens een hartkatheterisatie bij vrouwen bijna 2 keer vaker geen grote vernauwingen in de kransslagaders gezien worden dan bij mannen (39\% t.o.v. 23\%). In de jaren na deze katheterisatie, waarbij dus geen belangrijke vaatvernauwingen werden gezien, houdt de meerderheid van deze individuen toch klachten. Dit betreffen veelal klachten van pijn op de borst en klachten passend bij hartfalen. Maar ook psychische klachten komen veel voor. Vervolgens is gekeken of deze mannen en vrouwen méér klachten hebben dan de gemiddelde bevolking. Hiervoor is deze groep vergeleken met mannen en vrouwen van dezelfde leeftijd, uit dezelfde huisartsenpraktijk, die geen hartkatheterisatie nodig hadden. Alle klachten bleken vaker voor te komen in onze studiepopulatie dan in de gewone bevolking. Dit leert ons dat, ondanks dat de kransslagaders geen afwijkingen lieten zien, er mogelijk toch een vorm van hart- en vaatziekten speelt waardoor deze mannen en vrouwen klachten blijven houden. 
Behalve dat het verhoogde risico op het ontwikkelen van langdurige klachten en hartfalen natuurlijk ernstig lijden met zich meebrengt voor deze mannen en vrouwen zelf, gaat dit ook zonder meer gepaard met enorme economische en maatschappelijke (zorg)kosten. Daarom is het belangrijk dat er meer onderzoek gedaan wordt naar deze langdurige ziektelast en hoe deze kan worden verlaagd.

De voorgenoemde verstoorde functie van de microcirculatie is aldus een toenemend probleem. De laatste aanbevelingen van de Coronary Vasomotor Dysfunction studiegroep geeft richting bij wie, wanneer en welke onderzoeken gedaan moeten worden naar deze vorm van coronairlijden om deze zo vroeg mogelijk te kunnen diagnosticeren. Dit betreffen testen tijdens een hartkatheterisatie om de functie van de bloedvaten te onderzoeken. De eerste onderzoeken wijzen uit dat deze aanbevelingen goed uitvoerbaar zijn in de klinische praktijk en dat het uitvoeren van deze vaatfunctietesten zorgt voor minder klachten op lange termijn. Het nadeel is dat de aanbevolen onderzoeken belastend zijn voor de patiënt en de uitvoering ervan niet zonder risico is. Daarom is in hoofdstuk $\mathbf{9}$ gekeken in de literatuur of er minder belastende onderzoeken zijn om deze mannen en vrouwen met een verhoogd risico op slechte uitkomsten vroegtijdig te herkennen. Op dit moment is er geen nietinvasieve methode om de functie van de microcirculatie te bepalen. Wel is de hoeveelheid niet-invasieve beeldvormende technieken die in ontwikkeling zijn om de cardioloog in de toekomst te helpen met het stellen van een diagnose van coronair microvasculair lijden hoopgevend.

\section{Conclusie}

Dit proefschrift toont het belang aan om mannen en vrouwen met klachten verdacht voor coronairlijden apart te bestuderen. Deze sekse-specifieke benadering is van belang over de hele breedte van de zorg voor mannen en vrouwen met hart- en vaatziekten; van het beoordelen van symptomen, het toepassen van risicoscores tot aan het kiezen en interpreteren van het juiste aanvullende (beeldvormend) onderzoek. Alleen wanneer het individu wordt bekeken door de juiste 'sekse-specifieke bril' kan elk individu optimale zorg, diagnostiek en behandeling worden geboden. Mannen en vrouwen zijn niet gelijk, dus laten we ze gelijkwaardig behandelen. 


\section{Dankwoord}

Onderzoek doe je niet alleen en dit proefschrift was er niet geweest zonder hulp van collega's en vrienden om af en toe stoom bij af te blazen. Hieronder wil ik graag een aantal mensen in het bijzonder bedanken. Uiteraard is dit allesbehalve volledig dus iedereen die een bijdrage heeft geleverd aan dit proefschrift maar niet genoemd staat: Bedankt!

Allereerst natuurlijk alle patiënten en studie deelnemers zonder wie onderzoek niet mogelijk zou zijn; deelnemers van de ARGUS studie, patiënten van $C C N$ en deelnemers van de UCORBIO studie: bedankt voor jullie belangeloze inzet!

Prof. dr. ir. den Ruijter, beste Hester, wat ben ik trots dat ik jou mag bedanken als promotor. Bij mijn sollicitatie kwam jij later aanschuiven, minder misleidend had onze eerste kennismaking niet kunnen zijn. Je bent de afgelopen jaren mijn rots in de branding maar ook ultieme voorbeeld geweest, uiteraard als onderzoeker maar veel meer nog als mens. Dat dingen soms moeten schuren en wringen, nou dat heb ik geweten. Je hebt me uit mijn comfortzone getrokken, aangemoedigd onderuit te durven gaan en geleerd dat als rechtdoor niet lukt, er altijd een andere weg is om tot een doel te komen. Je vertelde mij dat de belangrijkste les uit mijn promotie op persoonlijk vlak zou zijn, je had het niet beter kunnen voorspellen. Bedankt dat je altijd achter me stond en nooit aan mij hebt getwijfeld, ook al deed ik zelf niet anders, je was een fantastische promotor.

Prof. dr. Leiner, beste Tim, bedankt voor je betrokkenheid bij zowel mijn promotie als het vormgeven van mijn verdere carrière. Ondanks je drukke agenda en verre reizen was je altijd te bereiken als dat nodig was. Bedankt dat je ook de tijd nam voor een kopje koffie om mee te denken hoe ik mezelf kon ontwikkelen en kon ontdekken waar mijn hart lag. Dankzij jou ga ik goed voorbereid en geïnspireerd beginnen bij de radiologie en ik hoop dat we ooit collega's zullen zijn. Bedankt voor het zijn van een hele fijne promotor en dat je me hebt laten zien dat het mogelijk is om zowel een échte wetenschapper te zijn als dokter.

Dr. Onland-Moret, beste Charlotte, ik ben heel dankbaar dat jij gedurende mijn promotie aan mijn begeleidingsteam bent toegevoegd. Je was er altijd om door alle bomen het bos weer te zien en structuur aan te brengen in de projecten. Dank voor je eindeloze geduld om mijn statistische (of epidemiologische?) kennis bij te spijkeren en de tijd - die je niet had maar wel nam - om ook gewoon even gezellig te kletsen of een luisterend oor te bieden als ik ergens mee worstelde. Bedankt! 
Dr. Eikendal, beste Anouk, al heb je die plek meer dan verdiend, het voelt wel een beetje gek om jou hier als copromotor te bedanken. Met de smoes 'Anouk heeft verstand van MRI's' mocht ik je van Hester vragen mij te begeleiden, nou volgens mij heb je me sindsdien bij alle aspecten van zowel het promoveren als het leven geholpen, maar een scan hebben we nog nooit samen bekeken. Als arts-epidemioloog, mentor, 'studie-bazen-manager', collega maar bovenal vriendin heb je de afgelopen jaren tot een waar feestje gemaakt en ik hoop dat er nog heel veel brainstorm en therapeutische schommel 'meetings' volgen. Je wordt hoe dan ook een fantastische huisarts!

Graag wil ik de leden van de leescommissie, te weten prof. dr. ir. van der Schouw, prof. dr. Rutten, prof. dr. Pasterkamp, prof. dr. Velthuis en prof. dr. Maas bedanken voor het zitting nemen in mijn beoordelingscommissie en kritisch lezen van mijn proefschrift.

Ook wil ik alle coauteurs bedanken voor de mooie publicaties en prettige samenwerking. In het bijzonder Crystel, bedankt dat ik gebruik mocht maken van jouw mooie studie en je bereidheid ook tijdens je drukke klinische werkweek mee te denken, en dr. Somsen van Cardiologie Centra Nederland, voor de scherpe maar opbouwende feedback vanuit de praktijk.

Dan alle mede-promovendi en andere collega's waar ik de afgelopen drie jaar fijn mee heb samengewerkt en van heb mogen leren.

De "Young Talent Club", bedankt voor de gezelligheid, meetings en ervaringen in Amerika en vooral mijn peer-groepje (Veerle, Mark en Elise) voor de fijne gesprekken die ik zo vroeg in mijn promotie traject erg goed kon gebruiken. Gerard, dank dat ik mee mocht op deze reis en jij er sindsdien bent geweest om gewoon even te luisteren, me aan het denken te zetten, te triggeren ("ik weet nog niet of je wel echt een wetenschapper bent") en compleet uit mijn comfort zone te trekken. Volgens mij is het je gelukt, gezien het 'echte' lab paper' als resultaat!

Het UPOD team, Saskia bedankt voor het warme welkom, de momentjes om even te sparren over onderzoek en hulp bij het datamanagement samen met Enja en Suzan. En natuurlijk Mark, bedankt voor al je hulp met de data en het om sleutelen van mijn PC. Imo, we hebben weinig direct samengewerkt maar bedankt voor je oprechte interesse in mij sinds de $1^{\mathrm{e}}$ minuut van mijn sollicitatie, dat je er altijd was als ik iets van je wilde weten en spot-on feedback tijdens de ARGUS overleggen. Bram en 
Tjebbe, het was boeiend om eens de andere kant van het ARGUS project te zien, mochten jullie ooit rijk worden met de verkoop van het algoritme, vergeet niet wie er heeft geholpen met labelen!

All people of the Experimental Cardiology group and the Laboratory of Clinical Chemistry and Hematology, especially Ineke en Joukje, thank you for the warm welcome since my - literally - first day chasing sheep, presentations that I still don't completely understand and all the fun in between (especially the pub quiz by Ray which was even more difficult than understanding some of the Friday Morning Meetings...). Thank you all for being so welcoming even though I was not really a lab-rat. Dear members of the Asselbergs group, thank you for letting me join every once in a while to gain interesting insights and learn from you.

Daniek, ik weet niet hoe ik de studie had moeten uitvoeren zonder jou! Ondanks alle chaos die in de loop der jaren is ontstaan weet jij altijd het overzicht te bewaren met een brede lach op je gezicht. Dank voor de fijne samenwerking, gezelligheid, tijd en ruimte om over alles en iedereen te klagen (al had jij er nog véél meer last van dan $i k)$, en het feit dat je hoe druk je het ook had altijd klaar stond voor de studie. Dankzij jou hebben we nu een mooie ARGUS-Biobank waar hopelijk in de toekomst nog mooie experimenten mee gedaan kunnen worden. Aan jouw goede ideeën en doorzettingsvermogen zal het in ieder geval niet liggen! Naomi, Lianne, Merel, Evelyn en Mark bedankt voor alle gezelligheid op het lab en jullie tomeloze inzet voor de studie.

Naast het lab wil ik ook de rest van het enorme ARGUS studieteam bedanken. Allereerst het Trialbureau en de Beeldbewerking van de Divisie Beeld, de laboranten van zowel de MRI als de CT en de planning. In het bijzonder Seline, Laura, Nils, Marjan, Gerrit, Ramona, Diana, Ellen, Simone, Marieke, Saskia, Greet, Bernadette, Rianne, Marion, Jorgen, Esther, Annick, Gideon, Ronald, Merel, Daniel, Carla, Nick en Mark. Ondanks de drukte bij jullie op de afdeling stonden jullie open voor samenwerking met Anne-Mar en mijzelf, ook al zorgde dit voor extra werk voor jullie. Ook was er altijd ruimte om voor onze studie te schuiven of ons wat extra uit te leggen. De automaat-koffie zal ik niet missen, maar jullie gezelligheid wel! Verder wil ik nog in het bijzonder Niels bedanken, als ik weer eens als een naïeve puppy terugkwam met een plan van Tim, hielp jij me dit plan om te bouwen naar iets wat enigszins uitvoerbaar was. Je was mijn steunpilaar wat betreft de MRI en ik heb veel van je geleerd. MEDIS, Hans, David en Pieter, dank voor alle hulp met de software en de fijne samenwerking! ledereen van CCN die betrokken was bij ARGUS, zowel cardiologen, balie dames en studenten als echografisten, bedankt voor jullie enorme 
inzet om patiënten hun weg naar de studie te doen vinden. Roxana, ik hecht veel waarde aan onze fijne samenwerking bedankt voor de tijd en energie die je erin hebt gestopt om ARGUS samen te doen slagen. Natuurlijk ook Clara, iedereen van het Diakonessenhuis, Janneke en het HeartLife-team bedankt.

Hester's Devils (inmiddels omgedoopt tot Science Lovers), Ingrid, bedankt dat jij het lab en onze groep draaiende houdt, voor het organiseren van alle uitjes en alle dagelijkse gezelligheid. Michèle, it was not frequent, but I always left the lab smiling after running in to you, lots of luck with your baby! Elise, bedankt voor het broodnodige kopje koffie op de maandag ochtend tijdens de weekstart, de yogaschilder-brainstormdagen en gezelligheid. Gideon, bedankt dat jij het wiel had uitgevonden voor ik begon (inclusief alle ovale of vierkante niet-rollende wielen), Robin, bedankt voor de kopjes koffie om gewoon even bij te kletsen of een tevergeefs - bijlesje genetica, heel veel succes met de rest van je carrière ik hoop je ooit in Amerika of Japan te mogen opzoeken! And Ernest, even though it was short, I am glad that I got to work with you. Thank you for your help, patience, and kindness with our project, you saved me by finishing it! We are very lucky with you in our team.

Ik wil ook de studenten bedanken die ik (deels) heb mogen begeleiden. Thuur, wat ben ik blij dat jij het meteen in mijn eerste maanden aandurfde samen met mij. Ik hoop dat jij net zoveel van mij hebt geleerd als ik van jou en ook de leuke kanten van het onderzoek hebt meegenomen naar de rest van je carrière. Ik kijk nog steeds uit naar je boek over het torenleven! De Troostpot was nooit meer hetzelfde zonder jouw veel te zure snoepjes. Mirna, bedankt dat ik jou mocht helpen je prachtige onderzoek af te ronden, het is een stuk geworden waar je trots op kunt zijn. Succes met de rest van je carrière! Milena, helaas had Sophie je al snel weggekaapt, maar het was altijd gezellig koffie drinken en kletsen over niet-wetenschappelijke dingen, ik hoop dat we nog eens zullen samenwerken! Willemijn, het was kort maar krachtig, bedankt voor je hulp! Leila, bedankt dat ik je mocht begeleiden en ik kijk uit naar het afronden van je onderzoek. Mayra, wauw, wat heb jij een werk verzet in die korte tijd en wat ben ik blij dat ik de studie in jouw handen kan achterlaten.

Marian en Birgit, ik durf wel aan te nemen dat ook jullie zwarte boekje nog steeds ongeopend ligt te verstoffen, zullen we die als we oud en grijs zijn samen openen? Ik ben blij dat ik jullie die week echt heb leren kennen en er twee nuchtere (Eureka) buddy's voor het leven bij heb gekregen. Birgit, heel veel succes in Duitsland, je collega's en patiëntjes daar hebben mazzel met jou. Marian, Ik ben blij dat jij als colourful bird aan bent komen vliegen. Sindsdien mijn onmisbare koffie maatje of het nou op het lab, digitaal of buiten werk is, en ook die taart is er gelukkig toch nog 
gekomen (ook al heeft het de lab-wedstrijd niet gehaald). Bedankt dat je mij als PhD hebt geadopteerd in de ivoren-postdoc-toren en het einde van mijn PhD is zeker niet het einde van onze vriendschap.

Alle vroegere en huidige Torenbewoners, bedankt voor de afgelopen jaren aan koffiemomentjes en levenslessen. Ian, vanaf dag 1 mijn ultieme voorbeeld hoe het einde van je PhD eruit moet zien: geen stress, eindeloos je voorkant knutselen en dan toch gewoon een stock afbeelding kiezen omdat het kan, bedankt voor de gezelligheid, koffie momentjes en wijze lessen als Toren-oudste. Tim, bedankt voor de gezelligheid en ik vind het bewonderenswaardig hoe jij voor je eigen pad hebt gekozen hoe jammer het ook was dat je ons achterliet. Arjan, we hebben maar kort samen de toren bewoond maar gelukkig op de valreep toch nog echt samengewerkt! Bedankt voor de fijne samenwerking, dat je me hebt meegenomen in de wereld van de Athero-Express en jouw doorzettingsvermogen en management tips toen ik ons project echt niet meer zag zitten. Michael en Malin, jullie waren een verfrissende datascience wind in de stoffige Toren, dank voor alle gezelligheid en hulp als ik weer kwam aankloppen met mijn zoveelste R-error. Heel veel succes met het afronden van jullie PhD's en hopelijk kunnen we nog eens real-life een paas- of kerstbrunch reünie doen! Sander, bedankt voor de altijd leuke, interessante, soms wat wazige, tip van de dag of vaderlijke raad als we weer eens bij de nespresso machine stonden bij te komen. Anne-Mar, partner-in-studie-crime, helaas heeft onze real life samenwerking niet al te lang geduurd maar het was wel mega gezellig en fijn om samen de kar te trekken. Heel veel succes met het op de rails houden van je kleurrijke promotieteam en ik kijk nu al uit naar je prachtige HELPFulUP-thesis! Diantha, ik vond het heel gezellig met jou erbij in onze virtuele Toren en het is echt heel knap hoe jij vanuit huis alles zo snel geregeld hebt voor je nieuwe studie. Heel veel succes de komende jaren!

De \#0900-Trootspothotline, zonder jullie was dit proefschrift er zeker nooit gekomen. Of we nou met de bus naar Disney gingen en op de zwarte lijst van de personeelsvereniging terecht kwamen omdat we onze stoel niet wilden opgeven voor een jankend kind, of op de fiets naar Parijs zaten (Jonne waar was je nou..), dankzij jullie werd het harde werken af en toe onderbroken voor een wandeling of weekendje zonder e-mails en vooral heel veel lol. Jonne, ik kan een heel boekje volschrijven over alles waar ik jou voor moet bedanken, maar daar heb je als moeder gelukkig helemaal geen tijd meer voor. Overigens, eigenlijk moet je mij bedanken, want vanaf mijn eerste dag heb je op mij kunnen oefenen voor het moederschap en mij mogen opvoeden en helpen opgroeien in de wondere wereld van de wetenschap en tijdens mijn eerste baan. Ondanks dat ik 'net 14' was en jij 'al bijna getrouwd' klikte het meteen en mocht ik met jullie mee naar New York, hebben we samen de studie best aardig aan de praat 
gekregen en werd je middels eindeloze koffies, XXL-chocolate cookies en overprized thee echt mijn maatje. Bedankt voor alle wijze levenslessen en ik hoop dat we nog vele avonturen gaan beleven als je ooit weer een dagje zonder Eli kan. Sophie, toen ik de Toren binnen kwam lopen was de toon meteen gezet; 'welkom, als je maar niet denkt dat ik je analyses ga doen'. Gelukkig meende je hier niets van (je had nl. mijn studieprotocol allang geschreven) en was je de afgelopen jaren mijn steun en toeverlaat. Een plek als copromotor zou zeker niet hebben misstaan. Ik ben blij dat we mijn hele periode in de Toren samen hebben mogen werken, koffie drinken, lachen en alle frustraties hebben kunnen delen. Ik hoop dat ik je kijk op arts-onderzoekers een beetje positiever heb kunnen maken. Je hebt me geïnspireerd een echte wetenschapper te willen worden en het plezier in het schrijven te ontdekken. Ik wens je heel veel succes met je nieuw job, maar voor jou is geen berg te hoog (zelfs niet op een rammelende tour fiets terwijl iedereen op een lichtgewicht carbon fiets omhoog sprint) dus met jouw doorzettingsvermogen en kracht om je eigen weg te volmaken kom je er wel. Ik zet je onvermijdelijke oratie als professor vast in mijn agenda over een paar jaar! Klaske, pfoe, dacht ik dat ik met Sophie een eenhoorn had getroffen als bewonderenswaardige collega waar ik me aan op kon trekken, kom jij aanzetten. In het begin was het wat zoeken met schijnbaar overlappende onderzoeksdoelen, maar uiteindelijk bleek het een gouden combinatie en werd onze samenwerking een perfect storm. Bedankt dat je altijd klaar stond om te zeuren, te sparren of een rondje - iets te hard - te fietsen. Succes met de laatste loodjes, maar na alle hobbels die jij hebt moeten nemen de afgelopen jaren zijn die peanuts. Vergeet je jezelf niet te bedanken als projectmanager, promotor en PhD in één? (Ex-) Torenmeiden, ik had me geen betere collega's en vriendinnen kunnen wensen, we vulden elkaar naadloos aan. Laten we de G\&T en sushi erin houden om onze successen te blijven vieren!

Lieve vriendinnen, gelukkig waren jullie er ook voor de nodige afleiding en om af en toe even objectief mijn PhD-gestress aan te horen. Altijd bleken mijn 'problemen' alles mee te vallen in het licht van de 'gewone wereld'. Olifanten werden weer muggen als ik ze hardop uitsprak tegen jullie. Jaim en Am wat ben ik blij dat we weer teamgenootjes zijn, ik was vergeten hoe fijn het is jullie weer zo vaak te zien en spreken! Daan, Nic, Linde en Joyce, ondanks onze korte samenspeel periodes zijn jullie vriendinnen voor het leven geworden. Char, samen van overijverige geneeskunde student tot echte dokters, laten we snel weer samen werken in hetzelfde ziekenhuis. Bedankt dat jullie er altijd zijn om even bij te kletsen, of het nou wekelijks is of er weer maanden niet van komt, het is altijd weer als vanouds en ik kan bij jullie voor alles terecht. 
Lieve $\mathbf{C u}$ en Niek, mijn beste maatjes, mijn rechter- en mijn linkerhand. Met jullie naast me kan ik alles aan en als ik, zo af en toe, aan mezelf twijfel zijn jullie er om me te vertellen dat ik even normaal moet doen. Als ik een mooie prestatie weer eens niet het vieren waard vind, staan jullie klaar met een te dure fles champagne of dropjes en ijs. Jullie zijn toppers en ik ben blij dat jullie altijd voor me klaar staan! Sorry Niek geen lofzang van een paar bladzijden maar je weet best hoe dankbaar ik je ben en je zou het toch niet woord voor woord lezen.

Als laatste wil ik natuurlijk mijn lieve familie bedanken. Zonder jullie onvoorwaardelijke steun en vertrouwen was ik nooit zo ver gekomen. Lieve opa Herman, dank dat u mijn artikelen altijd probeert te lezen ook al is het jargon niet te begrijpen, oma Bep en oma Miep, bedankt dat jullie me altijd verwennen als ik weer uit kom razen van de drukte van elke dag, dat zal de komende jaren nog meer dan nodig blijven en gelukkig werk ik weer een stuk dichterbij en opa Ruud ik zal uw onvoorwaardelijke trots altijd meenemen. Lief broer(tje), Kas, het is niet altijd makkelijk mijn broertje te zijn, maar ik ben blij dat je er bent en me af en toe helpt relativeren en doet inzien wat de echt belangrijke dingen in het leven zijn. Pap en Mam, als laatste een paar hele dikke zoenen voor jullie natuurlijk. Jullie hebben me altijd alle liefde, kansen en vrijheid gegeven te doen wat ik wil, me geholpen als dat kon en me een beetje afgeremd waar nodig. Daar ben ik jullie elke dag dankbaar voor. Bedankt dat jullie ons met jullie opvoeding alles hebben meegegeven om onze dromen na te jagen en geleerd hebben dat je met stroop meer bereikt dan met azijn, je met hard werken alles kan worden wat je wilt maar ook zeker dat er veel meer is in het leven dan werk. Papa, Mama, Kas en natuurlijk Niek, ik kan me geen fijner thuisfront wensen, bedankt voor alles! 


\section{List of Publications}

\section{Published}

Groepenhoff F, Ernest Diez Benavente, Boltjes A, Timmerman N, Waissi F, Hartman RJG, Onland-Moret NC, Pasterkamp G, den Ruijter HM. Plasma Testosterone Levels and Atherosclerotic Plaque Gene Expression in Men With Advanced Atherosclerosis. Frontiers in Cardiovascular Medicine, 2021

Groepenhoff F, Eikendal ALM, Rittersma, Gijsberts CM, Asselbergs FW, Hoefer IE, Pasterkamp G, Rutten FH, Onland-Moret NC, den Ruijter HM. Persistent Symptoms and Health Needs of Women and Men With Non-Obstructed Coronary Arteries in the Years Following Coronary Angiography. Frontiers in Cardiovascular Medicine, 2021

Groepenhoff F, Klaassen RGM, Valstar GB, Bots SH, Onland-Moret NC, den Ruijter HM, Leiner T, Eikendal ALM. Evaluation Of Non-Invasive Imaging Parameters In Coronary Microvascular Disease: A Systematic Review. BMC Medical Imaging, 2021

Groepenhoff F, Siegersma KR, Onland-Moret NC, Tulevski II, Hofstra L, Somsen GA, den Ruijter HM. NYHA Class Is Strongly Associated With Mortality Beyond Heart Failure In Symptomatic Women. EHJ: Quality Of Care And Clinical Outcomes, 2020

Groepenhoff F, Eikendal ALM, Bots SH, van Ommen AMLN, Overmars LM, Kapteyn D, Pasterkamp G, Reiber JHC, Hautemann D, Menken R, Wittekoek ME, Hofstra L, OnlandMoret NC, Haitjema S, Hoefer IE, Leiner T, den Ruijter HM. Cardiovascular Imaging Of Women And Men Visiting The Outpatient Clinic With Chest Pain Or Discomfort: Design And Rationale Of The ARGUS Study. BMJ Open, 2020

Groepenhoff F, Eikendal ALM, Onland-Moret NC, Bots SH, Menken FR, Tulevski II, Somsen GA, Hofstra L, den Ruijter HM. Coronary Artery Disease Prediction In Women And Men Using Chest Pain Characteristics And Risk Factors: An Observational Study In Outpatient Clinics. BMJ Open, 2020

Groepenhoff F, den Ruijter HM. Sex-Specific Thoracic Aortic Dimensions And Clinical Implications. Heart, 2020

Groepenhoff F, Bots SH, Kessler EL, Sickinghe AA, Eikendal ALM, Leiner T, den Ruijter HM. Sex-Specific Aspects In The Pathophysiology And Imaging Of Coronary MacroAnd Microvascular Disease. Journal of Cardiovascular Translational Research, 2020 
Valstar GB, Bots SH, Groepenhoff F, et al. Discovery Of Biomarkers For The Presence And Progression Of Left Ventricular Diastolic Dysfunction And Heart Failure With Preserved Ejection Fraction In Patients At Risk For Cardiovascular Disease. BMJ Open, 2019

Bots SH, Groepenhoff F, Eikendal ALM, Tannenbaum C, Rochon PA, Regitz-Zagrosek V, Miller VM, Day D, Asselbergs FW, den Ruijter HM. Adverse Drug Reactions To Guideline-Recommended Heart Failure Drugs In Women: A Systematic Review Of The Literature. JACC Heart Failure, 2019

Veerhoek D, Groepenhoff F, van der Sluijs MGJM, de Wever JWB, Blankensteijn JD, Vonk ABA, Boer C, Vermeulen CFW. Individual Differences In Heparin Sensitivity And Their Effect On Heparin Anticoagulation During Arterial Vascular Surgery. European Journal of Vascular and Endovascular Surgery, 2017

\section{Submitted}

Groepenhoff F, Siegersma KR, Eikendal ALM, op den Brouw WJ, Leiner T, Appelman Y, Tulevski II, Somsen GA, Onland-Moret NC, Hofstra L, den Ruijter HM. Coronary calcification measures predict mortality in symptomatic women and men.

\section{In preparation}

Groepenhoff F, Siegersma KR, Eikendal ALM, Leiner T, Rittersma ZH, Menken FR, Hofstra L, Onland-Moret NC, den Ruijter HM. Women with higher grades of coronary stenoses have lower calcium scores than men. 


\section{Curriculum vitae}

Floor Groepenhoff was born on 21 October 1994 in Amsterdam. She obtained her high school degree in 2012 at the Vossius Gymnasium in Amsterdam. That same year she started her medical studies at the VU University. During her medical studies, she was already fascinated by cardiovascular research. For her master thesis, she researched peri-operative anti-coagulation and the effect of deep hypothermic cardiac arrest according to coagulation processes. Besides marking her first steps in performing a clinical study, this internship

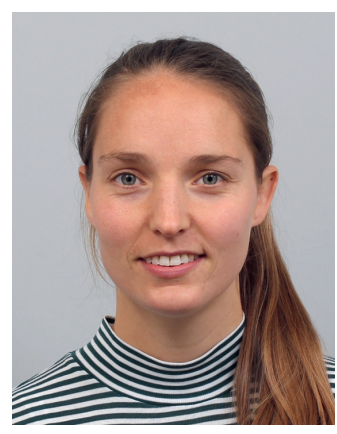
resulted in the publication of a peer-reviewed article and sparked her interest for an academic career. She completed the last year of medical school at the department of surgery of the Noord-West Ziekenhuisgroep and graduated as a medical doctor in May 2018. After a weekend of celebration, she started as a PhD Candidate at the University Medical Center Utrecht. Her main project comprised setting up and running the ARGUS study, which focused on extensive cardiovascular imaging in symptomatic women and men presenting at outpatient cardiology clinics. This was a collaboration between the Experimental Cardiology and the Radiology department. She was supervised by Prof. Hester den Ruijter, Prof. Tim Leiner, dr. Anouk Eikendal and dr. Charlotte Onland-Moret. The results of her research are presented in this thesis. 



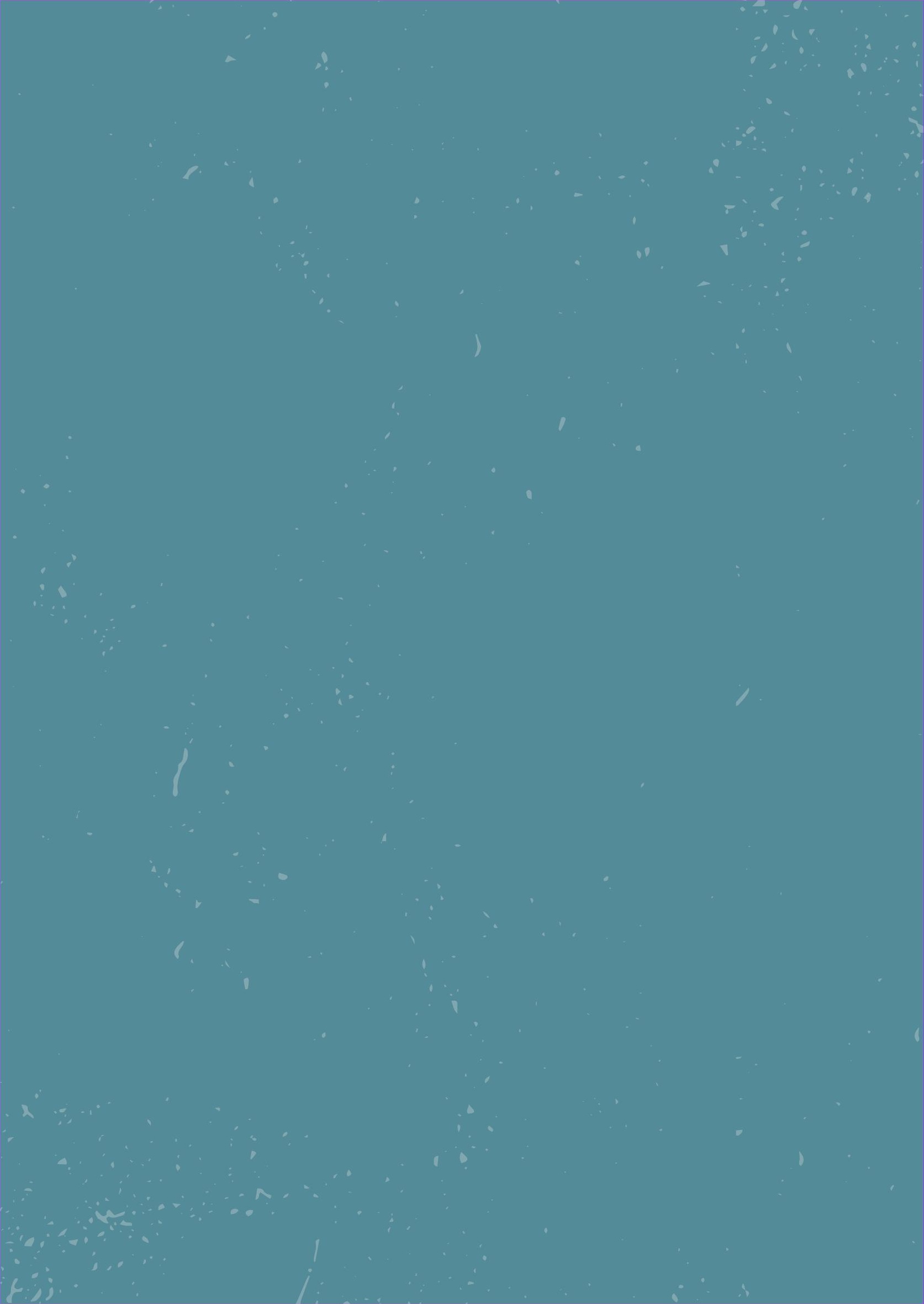

